\title{
A Measurement of Neutrino-Induced Charged-Current Neutral Pion Production
}

by

\section{Robert H. Nelson}

B.S., University of California, Santa Barbara, 2003

\author{
A thesis submitted to the \\ Faculty of the Graduate School of the \\ University of Colorado in partial fulfillment \\ of the requirements for the degree of \\ Doctor of Philosophy \\ Department of Physics
}

2010 
This thesis entitled:

A Measurement of Neutrino-Induced Charged-Current Neutral Pion Production written by Robert H. Nelson

has been approved for the Department of Physics

Prof. Eric D. Zimmerman

Prof. John P. Cumalat

Prof. William T. Ford

Prof. Kalyana T. Mahanthappa

Prof. Mitchell C. Begelman

Date

The final copy of this thesis has been examined by the signatories, and we find that both the content and the form meet acceptable presentation standards of scholarly work in the above mentioned discipline. 
Nelson, Robert H. (Ph.D., Physics)

A Measurement of Neutrino-Induced Charged-Current Neutral Pion Production

Thesis directed by Prof. Eric D. Zimmerman

This work presents the first comprehensive measurement of neutrino-induced charged-current neutral pion production $\left(\mathrm{CC} \pi^{0}\right)$ off a nuclear target. The Mini Booster Neutrino Experiment (MiniBooNE) and Booster Neutrino Beam (BNB) are discussed in detail. MiniBooNE is a high-statistics $\left(\sim 1,000,000\right.$ interactions) low-energy $\left(E_{\nu} \in\right.$ $0.5-2.0 \mathrm{GeV}$ ) neutrino experiment located at Fermilab. The method for selecting and reconstructing $\mathrm{CC} \pi^{0}$ events is presented. The $\pi^{0}$ and $\mu^{-}$are fully reconstructed in the final state allowing for the measurement of, among other things, the neutrino energy. The total observable $\mathrm{CC} \pi^{0}$ cross-section is presented as a function of neutrino energy, along with five differential cross-sections in terms of the final state kinematics and $Q^{2}$. The results are combined to yield a flux-averaged total cross-section of $\langle\sigma\rangle_{\Phi}=\left(9.2 \pm 0.3_{\text {stat. }} \pm 1.5_{\text {syst. }}\right) \times 10^{-39} \mathrm{~cm}^{2} / \mathrm{CH}_{2}$ at energy $965 \mathrm{MeV}$. These measurements will aid future neutrino experiments with the prediction of their neutrino interaction rates. 
Dedication

To my cat, Splinter. 


\section{Acknowledgements}

This work would not have been possible without the help and support of my advisor Eric Zimmerman, our postdoc Martin Tzanov, and graduate student Mike Wilking (as of this writing Mike is a postdoc at TRIUMF). Along the way there have been countless others who have supported my efforts, whether through direct advice and encouragement, or through the occasional libation. It would be improper for me not the mention Chivas Makaroplos for all the support she has shown me over the years.

On MiniBooNE, the help I received from Steve Brice, Sam Zeller, and Chris Polly drove this analysis to its conclusion. As this work is mostly based on the brilliant extended-track reconstruction, I must thank Ryan Patterson for doing the hard work before I started on this endeavour. Many, many people contributed to the success of both my analysis and the MiniBooNE experiment, too many to list here. However, it behooves me to mention all the graduate students who came before me as they showed me what the expectations were that I strived to live up to: Jen, Michel, Jocelyn, Ryan, Alexis, Chris, Dave, Serge, Teppei, Mike, Kendall, and Denis. In small and large ways you all directly influenced this work. Additionally I would like to acknowledge the support of Hiro Tanaka who, over countless glasses of scotch, provided me with excellent insight into physics, my analysis, and life as a whole. I wish the current round of MiniBooNE students the best of luck, writing a dissertation is a duanting task and looking back it will still seem to have been impossible task to have finished.

At Colorado there were countless ways to distract myself from the work I should 
have been doing and those distractions were usual caused by outside influences: Joe, Ryan, Chad, Heather, Molly, Rachel, multiple Brians, Keith, Jim, Josh, multiple Matts, Cotton, Tracy, and various other friends and acquaintances I've had the pleasure of having over the years.

Eric is an excellent advisor. He often let me work things out for myself rather than just give me the answer. His insight was invaluable whenever I became stuck on a problem where his advice was usually spot on, if not enough to point me in the right direction. Also, I must thank him for taking the time to thoroughly read this dissertation (twice) and make suggestions for improvement and catch typos.

Mike, Martin and I had countless arguments over physics, statistics, and whatever crazy ideas that one of us had. They were extremely useful exercises. I was more often wrong than right, and it was humbling to admit my mistakes.

At Fermilab I've had the pleasure of being friends with Chivas, Duncan, Backfish, Joe, many of the folks in the MCR, countless REU students, and the regulars at the UC. Many, many drinks were shared, and goodtimes had by all. I only wish that I could play the guitar as well as Backfish, Duncan, Derrek, and Darren. Chivas is the reason that many people made it through their respective programs, myself included, and I consider her to be one of the most valuable resources at Fermilab. She brought a certain level of sanity and normality to a place that is often more engrossed in the physics than it is in life.

Lastly I would like to acknowledge the support of my parents without whose support I would not have finished. The help of my brother Harry who was an invaluable resource for programming advice. And finally that of my girlfriend Licia. She asked me not to mention her, hence the dedication is to our cat, but I figured that she probably wouldn't ever read this far into my dissertation!

I apologize for any typos or grammatical mistakes in the above as this section is traditional not proofread by anyone. I guess I should also apologize for typos in the 
dissertation, though I've endeavoured to catch as many of those as I could. Lastly I would like to acknowledge anyone that I may have forgotten. If you are reading this, and I didn't mention you but probably should have, point it out to me and I will buy you a beer!

\footnotetext{
${ }^{1}$ Limited time offer for as long as I live, and we must be at the same physical location.
} 


\section{Contents}

\section{Chapter}

1 Introduction 1

1.1 Neutrinos in the Standard Model . . . . . . . . . . . . . . . . . . 2

1.1.1 Weak Interactions . . . . . . . . . . . . . . . . . . . 2

1.1.2 Neutrino Cross-sections . . . . . . . . . . . . . . . . . 2

1.1.3 Standard Model Extensions . . . . . . . . . . . . . . . . . . . 4

$1.2 \mathrm{CC} \pi^{0}$ Interactions $\ldots \ldots \ldots \ldots \ldots \ldots \ldots$

1.3 Dissertation Outline . . . . . . . . . . . . . . . . 8

2 MiniBoonE 9

2.1 Experimental Apparatus . . . . . . . . . . . . . . . . . . . . 9

$2.1 .1 \quad$ Primary Beamline . . . . . . . . . . . . . . . 10

2.1.2 Target and Horn . . . . . . . . . . . . . . . . . . . . . 11

2.1.3 Meson Decay Volume . . . . . . . . . . . . . . . . . . . . 16

2.1 .4 MiniBooNE Detector . . . . . . . . . . . . . . . . . 17

2.2 Simulations . . . . . . . . . . . . . . . . 26

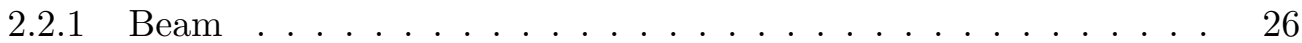

2.2 .2 Neutrino Flux . . . . . . . . . . . . . . . . . . . . . . 30

2.2 .3 Detector . . . . . . . . . . . . . . . . . . 32

2.3 Discussion . . . . . . . . . . . . . . . . . . . . . . 41 
3 The Little Muon Counter 43

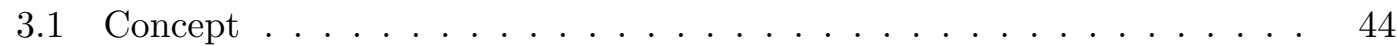

3.2 Fiber Hodoscope . . . . . . . . . . . . . . . . . . . . 46

$3.2 .1 \quad$ Apparatus . . . . . . . . . . . . . . . . . . 47

3.2.2 Booster and MiniBooNE Beam Extraction . . . . . . . . . . . . . 54

3.2 .3 Electronics and DAQ . . . . . . . . . . . . . . . 54

3.2 .4 Calibration ........................ 55

3.2 .5 Simulation . . . . . . . . . . . . . . . . 55

3.3 Event Reconstruction . . . . . . . . . . . . . . . . . . . . 59

3.3.1 Tracking Algorithm . . . . . . . . . . . . . . . 60

3.3.2 Momentum Reconstruction . . . . . . . . . . . . . . . . . 61

3.3.3 Fiber Plane Efficiencies _. . . . . . . . . . . . . . . . . 62

3.4 Analysis . . . . . . . . . . . . . . . . . . . . . 64

3.5 Discussion . . . . . . . . . . . . . . . . . . . 68

4 Event Reconstruction $\quad 69$

4.1 Single-Track Reconstruction . . . . . . . . . . . . . . . . . . . . . 70

$4.1 .1 \quad$ Event Topologies . . . . . . . . . . . . . . . . . . . 71

$4.1 .2 \quad$ Single-Track Likelihoods . . . . . . . . . . . . . . . . . . . . 74

$4.2 \mathrm{CC} \pi^{0}$ Reconstruction . . . . . . . . . . . . . . . . 78

$4.2 .1 \quad$ Event Topology . . . . . . . . . . . . . . . . . . 78

4.2 .2 Three-Track Likelihood . . . . . . . . . . . . . . . . . . 78

4.2 .3 Seeding the Fitter $\ldots \ldots \ldots$. . . . . . . . . . . 81

4.3 Fit Results . . . . . . . . . . . . . . . . . . . . . . . . 93

4.4 Neutrino Interaction Kinematics _ . . . . . . . . . . . . . . . . . 96

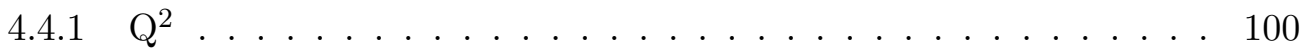

4.4 .2 Nucleon Resonance . . . . . . . . . . . . . . . . . . . . . . . . . 102 
4.5 Rejecting Misreconstructed Events . . . . . . . . . . . . . . . 103

4.6 Final Thoughts . . . . . . . . . . . . . . . . . . . . . . . . 107

$5 \quad \mathrm{CC} \pi^{0}$ Cross-sections and Analysis 110

5.1 Previous Measurements . . . . . . . . . . . . . . . . . . . 111

5.2 Measuring a Cross-section . . . . . . . . . . . . . . . . . . 113

5.3 The $\mathrm{CC} \pi^{0}$ Measurement . . . . . . . . . . . . . . . . 116

5.3.1 Defining the Signal . . . . . . . . . . . . . . . . . . . 117

$5.3 .2 \quad$ Cuts . . . . . . . . . . . . . . . . . . 118

5.3 .3 Event Rate . . . . . . . . . . . . . . . . . . . . . . . 127

5.3 .4 Backgrounds . . . . . . . . . . . . . . . . . . . . 127

5.3 .5 Unfolding . . . . . . . . . . . . . . . . . . . . . . 132

$5.3 .6 \quad$ Efficiency . . . . . . . . . . . . . . . . . . . 134

5.3 .7 Neutrino Flux . . . . . . . . . . . . . . . . . . . . . . . . 137

5.3 .8 Interaction Targets . . . . . . . . . . . . . . . . . . . . 138

5.4 Cross-section Uncertainties . . . . . . . . . . . . . . . . . . . . . 140

$5.4 .1 \quad$ Statistical Errors . . . . . . . . . . . . . . . . . . . . 143

5.4 .2 Flux Sources . . . . . . . . . . . . . . . . . . . . 145

5.4 .3 Detector Sources . . . . . . . . . . . . . . . . . . . . 146

5.4 .4 Systematic Error Discussion . . . . . . . . . . . . . . . 151

$5.5 \mathrm{CC} \pi^{0}$ Total Cross-section . . . . . . . . . . . . . . . . 152

$5.6 \mathrm{CC} \pi^{0}$ Differential Cross-sections $\ldots \ldots \ldots \ldots \ldots$

$5.6 .1 \quad Q^{2}$ Cross-section . . . . . . . . . . . . . . . . 154

5.6 .2 Muon Kinematic Cross-sections . . . . . . . . . . . . . . . . . . 154

5.6 .3 Pion Kinematic Cross-sections . . . . . . . . . . . . . . . . . 155

5.6.4 Flux-Averaged Total Cross-section . . . . . . . . . . . . . . 155

6 Conclusions 163 


\section{Appendix}

$\begin{array}{lr}\text { A Unfolding } & 171\end{array}$

A.1 Bayesian Unfolding . . . . . . . . . . . . . . . . . . . 172

A.2 Statistical Error Propagation . . . . . . . . . . . . . 173

A.3 Unfolding Closure Tests . . . . . . . . . . . . . . . . . . . . . . . 174

A.4 Multi-dimensional Unfolding . . . . . . . . . . . . . . 175

A.5 Remarks ........................ 177

B Cross-section Tabulations 178 


\section{Tables}

\section{Table}

2.1 The trigger rates, time windows, and prescales for each trigger type. . .

2.2 The Sanford-Wang parameters found by fitting $\pi^{ \pm}$production data [45, 46] and $K_{\mathrm{S}}^{0}$ production data $[45,47]$. The covariance matrices are found in Ref. [33]. . . . . . . . . . . . . . . . . . .

2.3 The Feynman scaling parameters found by fitting $K^{+}$production data [48, $49,50,51,52,53,54,55]$. The covariance matrix is found in Ref. [33].

2.4 Summary of the parameters describing the index of refraction in Marcol 7 found in Ref. [65]. . . . . . . . . . . . . . . . . . 37

3.1 Dominant decay modes with $\mu^{+}$in the final state for $\pi^{+}$and $K^{+}$. Branching fractions from Ref. $[20] . \ldots \ldots \ldots \ldots \ldots \ldots$

3.2 The alloy composition of Elgiloy. The average atomic number is, $\langle\mathrm{Z}\rangle=$ 27.41, and weight, $\langle\mathrm{A}\rangle=59.54$. The density, $\rho=6.345 \pm 0.439 \mathrm{~g} / \mathrm{cm}^{3}$, was measured using a buoyancy tank. . . . . . . . . . . . . . . . . . 49

3.3 The position of the fiber planes relative to the center of the dipole magnet. 51

3.4 The $x$-y dimensions of each individual range stack layer in inches. All the scintillators are 0.375 inches thick. Layer 8 is comprised of two sub-layers; one aligned vertically, the other horizontally. . . . . . . . . . . 53 
3.5 Non-standard GEANT3 material definitions: the material, average atomic number, average number of protons, density, and radiation length are listed. . . . . . . . . . . . . . . . . .

5.1 Expected number of events, efficiencies, and purities for each observable mode for each cut applied in succession. Additionally, the NUANCE fractions of the nucleon-level interactions that make up the observable $\mathrm{CC} \pi^{0}$ sample are shown for each cut. . . . . . . . . . . . . . .

5.2 The remaining background after all cuts by observable mode. Percentages are relative to the total amount of background. . . . . . . . . . . . .

5.3 The normalization uncertainties for all flux uncertainties. These uncertainties represent the uncertainties on the integrated flux. . . . . . . . 148

5.4 Parameters used in the optical model variations. Table reproduced from Ref. $[96] \ldots \ldots \ldots \ldots \ldots \ldots \ldots \ldots$

5.5 Normalization uncertainties on the observable $\mathrm{CC} \pi^{0}$ total cross-section measurement. . . . . . . . . . . . . . . . . .

5.6 Summary of the flux-averaged total cross-sections calculated from each cross-section measurement. The average cross-section is calculated assuming $100 \%$ correlated systematics. The flux-averaged neutrino energy

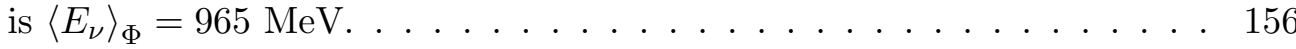

B.1 The total observable $\mathrm{CC} \pi^{0}$ cross-section as a function of neutrino energy (Fig. 5.16). Tabulated are the central value (CV), the total systematic error, the statistical error, and the correlation matrix for the systematic error defined by Eqn. 5.32. The correlations for the statistical errors are small and not tabulated. The CV, total systematic error, and statistical errors are multiplied by $10^{39}$. The bin boundaries are tabulated as the low edge of the bin with the last bin giving both the low and high edge. 
B.2 The flux-averaged observable $\mathrm{CC} \pi^{0}$ cross-section differential in $Q^{2}$ over the flux range $E_{\nu} \in(500-2000 \mathrm{MeV})$ (Fig. 5.17). Tabulated are the central value $(\mathrm{CV})$, the total systematic error, the statistical error, and the correlation matrix for the systematic error defined by Eqn. 5.32. The correlations for the statistical errors are small and not tabulated. The $\mathrm{CV}$, total systematic error, and statistical errors are multiplied by $10^{39}$. The bin boundaries are tabulated as the low edge of the bin with the last bin giving both the low and high edge. . . . . . . . . . . . .

B.3 The flux-averaged observable $\mathrm{CC} \pi^{0}$ cross-section differential in $E_{\mu}$ over the flux range $E_{\nu} \in(500-2000 \mathrm{MeV})$ in bins of $E_{\mu}-m_{\mu}$ (Fig. 5.18). Tabulated are the central value (CV), the total systematic error, the statistical error, and the correlation matrix for the systematic error defined by Eqn. 5.32. The correlations for the statistical errors are small and not tabulated. The CV, total systematic error, and statistical errors are multiplied by $10^{39}$. The bin boundaries are tabulated as the low edge of the bin with the last bin giving both the low and high edge. . . . . . . 18

B.4 The flux-averaged observable $\mathrm{CC} \pi^{0}$ cross-section differential in $\cos \theta_{\mu}$ over the flux range $E_{\nu} \in(500-2000 \mathrm{MeV})$ (Fig. 5.19). Tabulated are the central value $(\mathrm{CV})$, the total systematic error, the statistical error, and the correlation matrix for the systematic error defined by Eqn. 5.32. The correlations for the statistical errors are small and not tabulated. The CV, total systematic error, and statistical errors are multiplied by $10^{39}$. The bin boundaries are tabulated as the low edge of the bin with the last bin giving both the low and high edge. . . . . . . . . . . . 
B.5 The flux-averaged observable $\mathrm{CC} \pi^{0}$ cross-section differential in $\left|\mathbf{p}_{\pi^{0}}\right|$ over the flux range $E_{\nu} \in(500-2000 \mathrm{MeV})$ (Fig. 5.20). Tabulated are the central value $(\mathrm{CV})$, the total systematic error, the statistical error, and the correlation matrix for the systematic error defined by Eqn. 5.32. The correlations for the statistical errors are small and not tabulated. The CV, total systematic error, and statistical errors are multiplied by $10^{39}$. The bin boundaries are tabulated as the low edge of the bin with the last bin giving both the low and high edge. . . . . . . . . . . . . . . 183

B.6 The flux-averaged observable $\mathrm{CC} \pi^{0}$ cross-section differential in $\cos \theta_{\pi^{0}}$ over the flux range $E_{\nu} \in(500-2000 \mathrm{MeV})$ (Fig. 5.21). Tabulated are the central value $(\mathrm{CV})$, the total systematic error, the statistical error, and the correlation matrix for the systematic error defined by Eqn. 5.32. The correlations for the statistical errors are small and not tabulated. The CV, total systematic error, and statistical errors are multiplied by $10^{39}$. The bin boundaries are tabulated as the low edge of the bin with the last bin giving both the low and high edge. . . . . . . . . . . . . . . 184 


\section{Figures}

\section{Figure}

1.1 Left: Charged-current neutrino-nucleon scattering. Right: Neutralcurrent neutrino-nucleon scattering. . . . . . . . . . . . . 3

1.2 World data for charged-current (CC) neutrino interaction cross-sections [12]. 4

1.3 A tree level $\mathrm{CC} \pi^{0}$ Feynman diagram propagated through a nucleon resonance $N^{*} \ldots \ldots \ldots \ldots \ldots \ldots \ldots \ldots \ldots \ldots \ldots \ldots \ldots \ldots \ldots \ldots$

2.1 A cartoon of the MiniBooNE experiment. Pions and kaons are produced by interactions of protons on the beryllium target embedded in a magnetic focusing horn. The horn creates a toroidal magnetic field that focuses positively charged particles into the $50 \mathrm{~m}$ decay region. The particles decay-in-flight producing a forward neutrino beam. The beam passes through $500 \mathrm{~m}$ of earth before coming into contact with the MiniBooNE detector. Off-axis the secondary beam is a drift pipe that leads to the LMC enclosure $($ see Chapter 3$) \ldots \ldots \ldots$. . . . . . . . . . . . . . . . . 10

2.2 Drawing of the $8 \mathrm{GeV}$ transfer line off the Main Injector, the MiniBooNE primary beamline, target hall, and decay region. Schematic taken from Ref. $[31] \ldots \ldots \ldots \ldots \ldots \ldots \ldots$ 
2.3 Detail of the magnets in the $8 \mathrm{GeV}$ transfer line. The primary proton beam is transferred to the line through a kicker magnet at the top left. The beam passes through a series of focusing, de-focusing, and dipole magnets until it reaches the MI-12 surface building and the target. Protons that pass through the target impact an absorber 25 or $50 \mathrm{~m}$ downstream. Schematic taken from Ref. $[31] . \ldots \ldots \ldots \ldots$

2.4 The weekly number of protons delivered to the target from November 2002 through August 2008. Data in neutrino mode were taken 2/02/2003$1 / 19 / 2006$ and $10 / 14 / 2007-4 / 08 / 2008$ constituting $6.46 \times 10^{20}$ p.o.t. in neutrino mode. . . . . . . . . . . . . . . . . . . 14

2.5 A schematic of the target pile. The protons entered from the left. Schematic taken from Ref. $[32] . \ldots \ldots \ldots \ldots \ldots \ldots$

2.6 The beryllium target assembly. The target consists of 7 beryllium slugs for a total length of $71.1 \mathrm{~cm}$. Schematic taken from Ref. [33]. . . . . . .

2.7 The magnetic horn assembly shown with the cooling apparatus. The outer conductor is shown semi-transparent to see the inner conductor. The proton beam enters from the left. Schematic taken from Ref. [32], courtesy of Bartoszek Engineering $[34] \ldots \ldots \ldots \ldots \ldots$

2.8 The MiniBooNE detector schematic shown with a quarter sphere cut out. The PMTs are displayed as the yellow dots. The optically isolated veto region is shown in white. Schematic taken from Ref. [35]. . . . . . .

2.9 The MiniBooNE detector enclosure. The electronics plant sits above the detector. The oil plumbing system and overflow tank are on the right near the entrance to the enclosure. The slight dirt over-burden is apparent. Schematic taken from Ref. [35]. . . . . . . . . . . . . . .

2.10 The mapping of the placement of the new (R5912) and old (R1408) PMTs within the detector. Schematic taken from Ref. [35]. . . . . . . . . . 
2.11 The mounting brackets and inner wall that the tank PMTs attach to. The back to back placement of the veto PMTs is also apparent. Schematic taken from Ref. [35]. . . . . . . . . . . . . . . . . . . . . . . 21

2.12 The oil circulation system. Schematic taken from Ref. [35]. . . . . . . . 22

2.13 A PMT hit triggers an asynchronous discriminator that starts a voltage ramp, and immediately starts integrating the charge. The synchronous discriminator starts on the next clock tick after the trigger. Fast ADCs record the pulses $V_{t}$ and $V_{q}$ at 4 times, every $100 \mathrm{~ns}$, from $t-1$ through $t+2$. The total charge and initial time of the pulse are deduced from the FADC readout based on the fact that the shape of $V_{t}$ is fixed. Schematic taken from Ref. $[35] . \ldots \ldots \ldots \ldots \ldots \ldots$

2.14 The predicted neutrino flux for each species as a function of neutrino

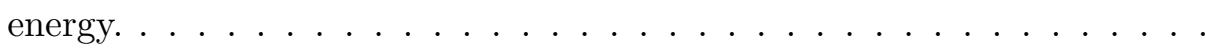

2.15 A charged particle passing through, or near a medium can radiate in the medium. The initial momentum of the charged particle is given by $p$, the final momentum by $p^{\prime}$. The radiated photon's momentum is given by $k$.

2.16 Optical photon extinction rates in Marcol 7. Extinction is referred to any process that alters the state of a photon. Wavelengths below $250 \mathrm{~nm}$ in the UV spectrum are not visible to the PMTs. Rayleigh scattering was measured at specific wavelengths and extrapolated by the known $\lambda^{4}$

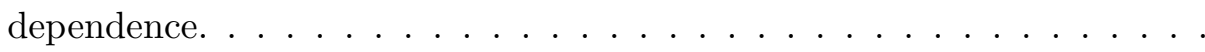

3.1 Left: The predicted $\nu_{\mu}$ flux separated by neutrino parent. Pion parents completely dominate the flux below $2.3 \mathrm{GeV}$, with kaon parents dominating at high energies. Right: The intrinsic $\nu_{e}$ flux separated by neutrino parent. The kaon parent contribution is significant across all energies and dominates above $1.2 \mathrm{GeV} \ldots \ldots \ldots \ldots \ldots \ldots$ 
3.2 The predicted energy spectrum of muons from pion parents (red) and kaon parents (blue) at $7^{\circ}$ off-axis the MiniBooNE beamline. . . . . . . .

3.3 Top view of the LMC. Muons come from the drift pipe (not shown) and enter a steel and tungsten collimator. On the face of the collimator is a scintillating veto. A fiber hodoscope measures the momentum of charge particles as they transit a dipole magnetic field with 6 planes of scintillating fibers. A secondary veto selects clean track through the hodoscope (MACC). Muons are selected based on their penetration depth into the range stack. A cartoon of a muon is shown in red. . . . . . . .

3.4 A photograph of the LMC. Muons enter from the left, through the collimator. The fiber tracker is shown with its light-tight lid removed to display the fiber planes and dipole magnet. . . . . . . . . . .

3.5 The timing of hits in fiber plane $1 \mathrm{X}$ separated by PMT modulo the bunch structure. The algorithm adjusts the last bunch to be less than 0 relative

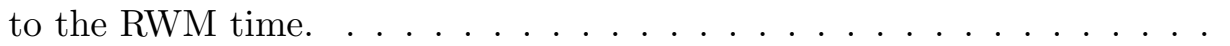

3.6 The timing structure of PMT \#48 in the fiber tracker at the beginning of the spill. The bunch structure is clearly visible. As only a few hits per PMT can be recorded during a single spill, a large sample of data is included in this plot. . . . . . . . . . . . . . . . . 56

3.7 GEANT3 renderings of several of the LMC simulated components. Active volumes are blue. Upper Left: Fiber planes 1X and 1Y. Upper Right: The veto counters. Bottom: The muon filter. The exposed face shows

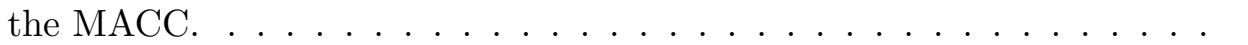

3.8 A simulation of a gas of muons incident the LMC collimator. Part of the collimator, the drift pipe, and part of the muon filter are not shown. . . 
3.9 Two $\mu^{+}$candidate events. The $\mathrm{X}$ fiber planes are scaled to be closer than reality in this view. The reconstructed track parameters have been adjusted according. The veto, MACC, and muon filter are not to scale and are included to show hits in the active areas. The colors in a plane denote separate hits. . . . . . . . . . . . . .

3.10 Projection of data events back to the front face of the collimator for well reconstructed $\mu^{+}$candidate events. Left: Any number of veto hits. Right: No veto hits. This shows the sensitivity to the fact that one of the veto counters was slightly in the beam. . . . . . . . . .

3.11 The reconstructed $\mu^{+}$momentum for $\pi^{+}$and $K$ parents based on the default beam MC. Resolution effects cause the distributions to smear into each other. Beam hadronic-interaction backgrounds are not included. Detector inefficiencies are included in the MC. This plot is the recon-

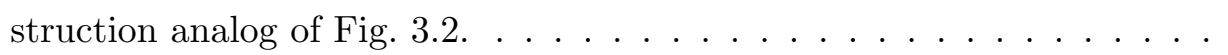

3.12 Left: Reconstructed momenta of a muon gas MC vs. penetration depth (range) into the range stack. The black lines define a cut to accept upwards of $95 \%$ of incident muons. All events between the lines are kept. Right: A pion gas MC over the same energy range as the muon gas. The pions range out much sooner than the muons. . . . . . . . . . . .

3.13 Reconstructed momentum vs. range for the LMC data set. The events separate into two clear regions; the muons within the muon-ID cut, and hadrons at high momentum and low range. . . . . . . . . . . . 66 
3.14 Left: The reconstructed momentum distribution for data and signal MC that pass all cuts. A significant difference is seen between data and MC (see text). Right: The reconstructed momentum distribution for data and signal $\mathrm{MC}$ after all cuts and restricted to range 8 in the muon filter. The errors are statistical on the MC. The data stats are insignificant in

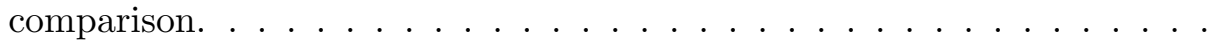

4.1 The MC true kinetic energy distributions for charged particles $\left(\mu, e / \gamma, \pi^{+}, p\right)$ weighted by their Čerenkov thresholds in mineral oil for events that make it into the $\mathrm{CC} \pi^{0}$ sample (except for $\pi^{+}$which come from $\mathrm{CC} \pi^{+}$events). Events to the right of the black line are above the Cerenkov threshold. The Čerenkov threshold for a charge particle in the mineral oil is approximately $0.32 \times$ mass. . . . . . . . . . . . . . . . . . . . 71

4.2 The angular profiles for PMT hits relative to the track directions for both muons (left) and electrons (right) for prompt (red) and late (blue) light for both data and MC. The muons have energies between 400 and $500 \mathrm{MeV}$ and the electrons are from stopped muon decays. The muon's angular distribution shows a sharp edge (at $\cos \theta \sim 0.7)$ of the Čerenkov ring as opposed to the electron's fuzzier edge. The apparent dip in the late light distributions are due to the dead time of the electronics. . . .

4.3 The distance between the one-track muon fit vertex and the true $\mathrm{CC} \pi^{0}$ vertex. Plotted are the number of events per proton on target (p.o.t.) per cm for signal events. . . . . . . . . . . . . . . .

4.4 The "angle" between the one-track muon fit and the true muon track in a $\mathrm{CC} \pi^{0}$ event. . . . . . . . . . . . . . . .

4.5 The smallest "angle" between the one-track muon fit and any track in a

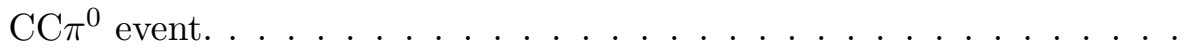


4.6 A roughly evenly spaced grid of 400 points on a sphere projected onto a flat surface, the left and right edges fold back onto each other. The coordinates, $\theta$ and $\phi$, are relative to the detector coordinates. The red dots are the angular directions of the axes: $+\hat{z}$ (beam) is the top point of the grid $(0,0)$, and $-\hat{z}$ is at the bottom $(0, \pi) ;+\hat{x}$ (beam left) is at both $(-\pi, \pi / 2)$ and $(\pi, \pi / 2) ;-\hat{x}$ (beam right) is the dead center of the plot, $(0, \pi / 2) ;+\hat{y}$ (vertical) is at $(-\pi / 2, \pi / 2)$ and the $-\hat{y}$ (downward) is at $(\pi / 2, \pi / 2) \ldots \ldots \ldots \ldots \ldots \ldots \ldots \ldots \ldots \ldots \ldots \ldots \ldots \ldots \ldots \ldots$

4.7 A likelihood scan in solid angle of a true $\mathrm{CC} \pi^{0}$ event. The red dot is the true muon direction relative to the one-track muon vertex. The brown dots are the directions of the true photons. The red cross is the result of the one-track muon fit. Finally, the yellow dot shows the location of the minimum of the likelihood surface. The one-track fit clearly found a photon; the likelihood scan found the other photon. . . . . . . . . .

4.8 After a two-track muon fit seeded by the results of the first scan, a second scan is performed. Comparing with Fig. 4.7 the surface shows a dimming of the photon peak and a brightening of the muon peak. The scan clearly finds the muon. The red crosses are the results of the two-track fit. . . .

4.9 A three-track muon fit is performed with the seed set by the second scan. This fit improves the event vertex and track directions. . . . . . . . .

4.10 The angle between the true muon direction and the Michel direction in slices of $50 \mathrm{~cm}$ over a range of $500 \mathrm{~cm}$. Plotted are the number of events per proton on target (p.o.t.). Each plot has a different range of angle in an attempt to plot the bulk of the distributions. $\ldots \ldots \ldots \ldots$ 
4.11 The true muon with respect to Michel angle versus the Michel distance. The curve is fit to twice the sigma above the mean gathered from the fits in Fig. 4.10. This choice was arbitrary, and was designed to penalize the Michel portion of the likelihood at small Michel distances. . . . . . . .

4.12 The fraction of events where the likelihood (Eqn. 4.34) chose the correct particle type configuration versus $\lambda$. The maximum of this curve is at

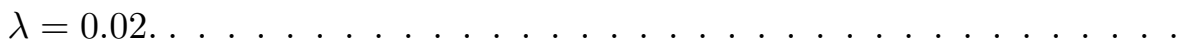

4.13 The final three-track fit with two of the muons swapped out for photons during the fit. The reconstructed photons are brown and match up with the true photons. . . . . . . . . . . . . . .

4.14 The angle between the fit muon and the true muon for both the $\mathrm{CC} \pi^{0}$ fitter and the muon-CCQE fitter (same curve as Fig. 4.4). A dramatic improvement over the muon-CCQE is apparent for both the muon yield and the muon angular reconstruction. . . . . . . . . . . . 96

4.15 The fit vertex versus true event vertex for both the $\mathrm{CC} \pi^{0}$ fitter and the muon-CCQE fitter. The fit shows a rather dramatic improvement where the bulk of the event vertices are within $50 \mathrm{~cm}$ of their true vertex. . . . 97

4.16 The muon fit kinematics along with the fit residuals. Top left: The kinetic energy for true and reconstructed events. Top center: The energy residual with an $8 \%$ energy resolution in the peak. Top right: The residual vs true kinetic energy. Bottom left: The angle wrt the beam direction for true and reconstructed events. Bottom right: The angle between the true and reconstructed directions. . . . . . . . . 
4.17 The first fit photon's kinematics along with the fit residuals. Top left: The energy for true and reconstructed events. Top center: The energy residual with an $19 \%$ energy resolution in the peak. Top right: The residual vs true kinetic energy. Bottom left: The angle wrt the beam direction for true and reconstructed events. Bottom center: The angle between the true and reconstructed directions. Bottom right: The reconstructed conversion length.

4.18 The second fit photon's kinematics along with the fit residuals. Top left: The energy for true and reconstructed events. Top center: The energy residual with an $28 \%$ energy resolution in the peak. Top right: The residual vs true kinetic energy. Bottom left: The angle wrt the beam direction for true and reconstructed events. Bottom center: The angle between the true and reconstructed directions. Bottom right: The reconstructed conversion length. . . . . . . . . . . . . . . . . 100

4.19 The $\pi^{0}$ fit kinematics along with the fit residuals. Top left: The magnitude of the momentum for true and reconstructed events. Top center: The momentum residual with an $14 \%$ momentum resolution in the peak. Top right: The residual vs true momentum. Bottom left: The angle wrt the beam direction for true and reconstructed events. Bottom center: The angle between the true and reconstructed directions. Bottom right: The reconstructed $\pi^{0}$ mass. . . . . . . . . . . . . . . . 101

4.20 A tree level $\mathrm{CC} \pi^{0}$ Feynman diagram propagated through a $\Delta^{+}$nucleon resonance. . . . . . . . . . . . . . . . . . . . . . . . 101

4.21 Left: The neutrino energy for true and reconstructed $\mathrm{CC} \pi^{0}$ events. Center: The neutrino energy resolution for all energies. The overall resolution is $11 \%$ in the peak. Right: The energy resolution as a function of true neutrino energy. . . . . . . . . . . . . . . . . . . . 102 
4.22 Left: The 4-momentum transfer, $Q^{2}$, for true and reconstructed $\mathrm{CC} \pi^{0}$ events. Center: The $Q^{2}$ resolution. The overall resolution is $29 \%$ in the peak. This distribution is subject to discrete misreconstruction. Right: The $Q^{2}$ resolution as a function of true $Q^{2}$. The bulk of events below -0.6 residual are from misidentifying the muon. . . . . . . . . . . . . . 102

4.23 The reconstructed nucleon resonance peaks at $1240 \mathrm{MeV} / c^{2}$, very close to the $\Delta(1232)$ mass with a full width of $168 \mathrm{MeV} / c^{2} \ldots \ldots \ldots$

4.24 The smallest angle between the three reconstructed tracks for events that correctly identified the particle configuration (found $\mu$ ) and those that did

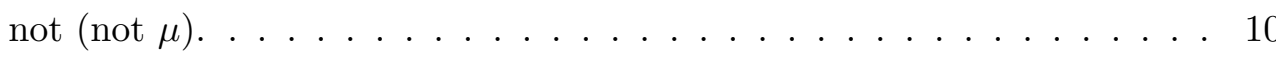

4.25 The optimization parameter $N / \sqrt{F+N}$ versus the smallest angle between reconstructed tracks. The parameter in each bin is found by integrating the numerator and denominator separately for every bin in Fig. 4.25 with angles less than itself, including itself. The line is set at the bin boundary where cutting events to the left of the line removes the optimal number of misreconstructed events. . . . . . . . . . . . . . 105

4.26 The muon kinetic energy after the misreconstruction rejection cut. The agreement between the true and reconstructed distributions is fairly remarkable. While the overall energy is unchanged, the obvious misreconstruction features found in Fig. 4.16 have been significantly reduced. . . 106

4.27 The neutrino energy and $Q^{2}$ resolutions after the misreconstruction rejection cut. The neutrino energy resolution is unaffected by the cut. The $Q^{2}$ resolution is improved to $25 \%$ overall, with the discreet misreconstruction effect seen in Fig. 4.22 at negative values significantly reduced. . . . . . 107 
4.28 The reconstructed $\gamma \gamma$ mass after the misreconstruction rejection cut. The distribution peaks at $139 \mathrm{MeV} / c^{2}$ with a width of $47 \mathrm{MeV} / c^{2}$. The width is entirely due to smearing. The line shows the position of the world average $\pi^{0}$ mass $[20] \ldots \ldots \ldots \ldots \ldots$. . . . . . . . . . . 108

5.1 The world data for $\mathrm{CC} \pi^{0}$ production. That data are from Refs. [85, 86, 87, 88, 89]. Only the ANL and BNL experiments measured $\mathrm{CC} \pi^{0}$ interactions at MiniBooNE energies. Both those experiments used a deuterium target. The models shown underpredict the data and are the NUANCE [57] and NEUGEN [90] models. . . . . . . . . . . . . . .

5.2 For a single neutrino interaction the number of PMT hits is plotted as a function of the time of the hit. The first cluster falls within the $1.6 \mu$ beam window and is called the first "subevent." The second cluster is due to a stopped muon decay and is called the second "subevent." . . .

5.3 The one-track fits likelihood ratio as a function of fit muon kinetic energy. CCQE events are shown in blue and populate the top of the plot. Overlayed are $\mathrm{CC} \pi^{0}$ events, in red, which tend to look more electron-like. The line, defining the $\mathrm{CC} \pi^{0}$ filter, shows the optimum position for a cut to keep $\mathrm{CC} \pi^{0}$ events and reject CCQE events. . . . . . . . . . . . .

5.4 The smallest angle between the three reconstructed tracks for both data (black) and central value MC (blue). Backgrounds are included in the MC. The MC is also broken up into events that correctly identified the muon (red), according to the particle-ID, and those that do not (green). Data are shown with statistical errors. Both samples have fiducial, tank and veto hits, and $\mathrm{CC} \pi^{0}$ event filter cuts applied. The black line shows the position of the cut to reject misreconstructed events found in $\S 4.5$. Events to the left of the line are cut. . . . . . . . . . . . . . 12 
5.5 Left: The logarithm of the ratio of the final fit likelihood compared with the generic three-muon fit likelihood for both data (black) and central value MC (blue). The MC is split into signal events (red), background events with $\pi^{0}$ (brown), and backgrounds without $\pi^{0}$ (green). The line optimizes the cut to reject backgrounds without $\pi^{0}$ while preserving signal, events to the left are cut. Right: Non- $\pi^{0}$ background rejection cut optimization. Backgrounds with $\pi^{0}$ are not including in the optimization as they look extremely similar to signal. . . . . . . . . . . . .

5.6 Center: The reconstructed $\gamma \gamma$ mass. The MC is separated into signal (red), backgrounds with $\pi^{0}$ in the final state (brown), and backgrounds without $p i^{0}$ (green). The lines represent the location of the optimized cuts, with the region around the obvious $\pi^{0}$ mass kept. Left: Optimization of the low mass cut to reject non- $\pi^{0}$ events from signal. Events to the left of the line are cut. Right: Optimization of the signal over all backgrounds. Events to the right of the line are cut. . . . . . . . . .

5.7 The measured event rate in neutrino energy for data and total MC. Data is shown with Poisson statistical error bars. . . . . . . . . . . . . .

5.8 The reconstructed $\gamma \gamma$ mass by observable mode. Each mode is represented after all cuts (including the mass cut). The observable $\mathrm{CC} \pi^{0}$ dominates the sample with $\mathrm{CC} \pi^{+}$as the largest source of background. The $\mathrm{CC} \pi^{+}$events have been reweighted according to the method described in $\$ 5.3 .4 .1 \ldots \ldots \ldots \ldots \ldots \ldots \ldots \ldots$

5.9 Ratio of data over $\mathrm{MC}$ for the observable $\mathrm{CC} \pi^{+}$cross-section as measured in Ref. [71]. This ratio forms the reweighting that is applied to each observable $\mathrm{CC} \pi^{+}$event in the $\mathrm{CC} \pi^{0}$ event sample. . . . . . . . . . 132

5.10 The Bayesian unfolding matrix for neutrino energy. . . . . . . . . . . . 134 
5.11 The bin efficiency for signal events to pass all cuts as a function of neutrino energy. The errors are statistical on the MC and are appropriately correlated. The total efficiency is $6.4 \% \ldots \ldots \ldots \ldots$

5.12 The predicted muon neutrino flux as a function of neutrino energy presented in bins and over the range of this analysis. The band shows the total systematic error. The flux-averaged neutrino energy is $\left\langle E_{\nu}\right\rangle_{\Phi}=965$ MeV. .......................... 137

5.13 Relative flux uncertainties for $\pi^{+}$production, $K^{+}$production, and the set of beam unisims. The $K^{+}$errors dominate at energies above this range, and the $\pi^{+}$errors get really large below $400 \mathrm{MeV}$. The error matrices are added in succession, with the beam unisim errors representing the total

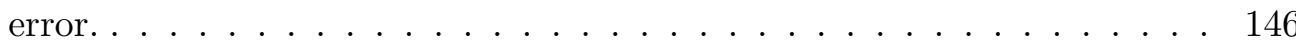

5.14 The correlation matrix for $\pi^{+}$production defined by Eqn. 5.32. While some bins are highly correlated, the maximum anti-correlation is around

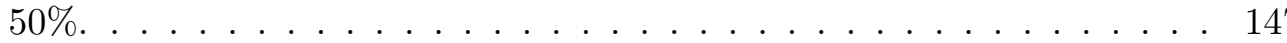

5.15 The cross-sections for $\pi^{+} \rightarrow \pi^{0}$ (left) and $\pi^{+} \rightarrow \Varangle$ (right) off carbon as a function of $\pi^{+}$kinetic energy. Data are from Ref. [97]. The hadronic models, GFLUKA [98] and GCALOR [99], seem to prefer GCALOR to match the data. The uncertainties are set by the data. . . . . . . . . . . . . . . 151 
5.16 Top: The total observable $\mathrm{CC} \pi^{0}$ cross-section as a function of neutrino energy. Each error band is plotted in a stacked fashion where all error matrices with a smaller normalization error is added to labeled error band. Beam $\mathrm{K}^{0}$ production has the smallest normalization component, and $\pi^{+} \rightarrow \pi^{0}$ and $\pi^{+}$absorption is the largest error and represents the total systematic error. The flux-averaged cross-section over the range $E_{\nu} \in(500-2000 \mathrm{MeV})$ is $\langle\sigma\rangle_{\Phi}=(9.05 \pm 1.44) \times 10^{-39} \mathrm{~cm}^{2} / \mathrm{CH}_{2}$. Bottom: The relative errors found by dividing out the measured crosssection. The total systematic normalization error is $18.7 \%$. . . . . . 157

5.17 Top: The flux-averaged observable $\mathrm{CC} \pi^{0}$ cross-section differential in $Q^{2}$ over the flux range $E_{\nu} \in(500-2000 \mathrm{MeV})$. The total flux-averaged cross-section calculated by integrating over $Q^{2}$ is $\langle\sigma\rangle_{\Phi}=(9.28 \pm 1.50) \times$ $10^{-39} \mathrm{~cm}^{2} / \mathrm{CH}_{2}$. Bottom: The relative cross-section error. The total systematic normalization error is $16.1 \% \ldots \ldots \ldots \ldots \ldots$

5.18 Top: The flux-averaged observable $\mathrm{CC} \pi^{0}$ cross-section differential in $E_{\mu}$ over the flux range $E_{\nu} \in(500-2000 \mathrm{MeV})$. The total flux-averaged cross-section calculated by integrating over $E_{\mu}$ is $\langle\sigma\rangle_{\Phi}=(9.20 \pm 1.47) \times$ $10^{-39} \mathrm{~cm}^{2} / \mathrm{CH}_{2}$. Bottom: The relative cross-section error. The total systematic normalization error is $15.8 \% \ldots \ldots \ldots \ldots$. . . . . . . . 159

5.19 Top: The flux-averaged observable $\mathrm{CC} \pi^{0}$ cross-section differential in $\cos \theta_{\mu}$ over the flux range $E_{\nu} \in(500-2000 \mathrm{MeV})$. The total fluxaveraged cross-section calculated by integrating over $\cos \theta_{\mu}$ is $\langle\sigma\rangle_{\Phi}=$ $(9.10 \pm 1.50) \times 10^{-39} \mathrm{~cm}^{2} / \mathrm{CH}_{2}$. Bottom: The relative cross-section error. The total systematic normalization error is $17.4 \%$. . . . . . . . 160 
5.20 Top: The flux-averaged observable $\mathrm{CC} \pi^{0}$ cross-section differential in $\left|\mathbf{p}_{\pi^{0}}\right|$ over the flux range $E_{\nu} \in(500-2000 \mathrm{MeV})$. The total fluxaveraged cross-section calculated by integrating over $\left|\mathbf{p}_{\pi^{0}}\right|$ is $\langle\sigma\rangle_{\Phi}=$ $(9.03 \pm 1.54) \times 10^{-39} \mathrm{~cm}^{2} / \mathrm{CH}_{2}$. Bottom: The relative cross-section error. The total systematic normalization error is $15.9 \%$. . . . . . . . . 161

5.21 Top: The flux-averaged observable $\mathrm{CC} \pi^{0}$ cross-section differential in $\cos \theta_{\pi^{0}}$ over the flux range $E_{\nu} \in(500-2000 \mathrm{MeV})$. The total fluxaveraged cross-section calculated by integrating over $\cos \theta_{\pi^{0}}$ is $\langle\sigma\rangle_{\Phi}=$ $(9.54 \pm 1.55) \times 10^{-39} \mathrm{~cm}^{2} / \mathrm{CH}_{2}$. Bottom: The relative cross-section error. The total systematic normalization error is $16.3 \% \ldots \ldots \ldots$. . . . 162

A.1 Top left: The true, reconstructed, and unfolded $E_{\nu}$ distributions. The unfolded distribution is identical to the true distribution as expected. Top Right: The bin migration matrix formed by the transpose of the column normalized response matrix. Bottom Left: The Bayesian unfolding matrix defined as the row normalized response matrix. Bottom Right: The response matrix. . . . . . . . . . . . . . . . 175

A.2 Right: The product of the unfolding times the bin migration matrix. Left: The true distribution and the unfolding times bin migration times the true distribution. The true distribution is an eigen-distribution of

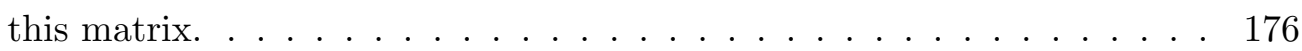




\section{Chapter 1}

\section{Introduction}

Neutrinos are the cleanest probe of nuclear matter. Neutrinos are light, electricallyneutral particles that do not interact through the strong nuclear force. When encountering nuclear matter, they penetrate deeply into a nucleon before occasioning a weak interaction after which they either continue on as neutrino never again interacting in our galaxy (or universe for that matter), or they change into their charged lepton partner and interact with the medium around them. The properties that make neutrinos such good probes of nucleons also make them extremely difficult to work with. The first evidence for neutrinos came about as a desperate solution by Pauli to conserve energy in beta decays [1]. They were formally introduced into the theory by Fermi [2]. Neutrinos existed as a curiosity for many years. They were first detected at the Savannah River nuclear reactor in South Carolina by Reines and Cowan in 1956 [3]. For a long time afterward, the neutrino was of mild curiosity in the standard model of particle physics, rarely interacting and hard to detect. There was a slight problem with the expected number of electron anti-neutrinos coming from the sun that disagreed with experiment $[4,5,6]$, but the chances were either the prediction was off, or the experiment was inaccurate. 


\subsection{Neutrinos in the Standard Model}

Neutrinos are the only particles in the Standard Model (SM) that interact only through the weak force. They carry zero electric charge and are assumed to be massless. As the weak force maximally violates parity, the handedness of the neutrino is fixed and they are all left handed. It is not possible to create a right-handed neutrino nor is it possible for it to interact in the SM. Additionally, there are three known neutrino species and each is paired with one of the three charged leptons $(e, \mu, \tau)$.

\subsubsection{Weak Interactions}

Neutrino interactions are governed by the Lagrangians given by $[7,8,9,10]$

$$
\begin{aligned}
-\mathcal{L}_{C C} & =\sum_{l=e, \mu, \tau} \frac{g}{\sqrt{2}}\left(\bar{\nu}_{l L} \gamma^{\mu} e_{l L} W_{\mu}^{+}+\bar{e}_{l L} \gamma^{\mu} \nu_{l L} W_{\mu}^{-}\right) \\
-\mathcal{L}_{N C} & =\sum_{l=e, \mu, \tau} \frac{g}{2 \cos \theta_{W}} \bar{\nu}_{l L} \gamma^{\mu} \nu_{l L} Z_{\mu}^{0}
\end{aligned}
$$

where $\mathcal{L}_{C C}$ is the charged-current Lagrangian that couples neutrinos and anti-neutrinos

with a $W^{ \pm}$boson into their charged lepton partners and vice versa, $\mathcal{L}_{N C}$ is the neutralcurrent Lagrangian that couples neutrinos and anti-neutrinos with the $Z^{0}$ boson, $g$ is the weak coupling constant, $\theta_{W}$ is the Weinberg angle, $\left(\bar{\nu}_{l L}\right)$ are the spinors representing the (anti)neutrinos of flavor $l$ and left-handedness $L$, and $\left(\bar{e}_{l L}\right)$ are the spinors representing the charged leptons. These are the only possible neutrino interactions in the SM.

\subsubsection{Neutrino Cross-sections}

The CC and NC neutrino interaction terms in the SM Lagrangian are exactly calculable. However, these processes only occur during neutrino interactions with matter. If these interactions are with leptons, the full process and therefore cross-section is exactly calculable. Additionally, identical terms exist for the quarks, and at high interaction energies when the quarks are asymptotically free, the scattering off bare quarks 
is calculable. However, at lower energies where the neutrinos interact with bound nucleons or the nucleus as a whole, strong interactions prevent the hadronic current from being exactly calculable. Fig. 1.1 shows Feynman diagrams for $\mathrm{CC}$ and $\mathrm{NC}$ inclusive
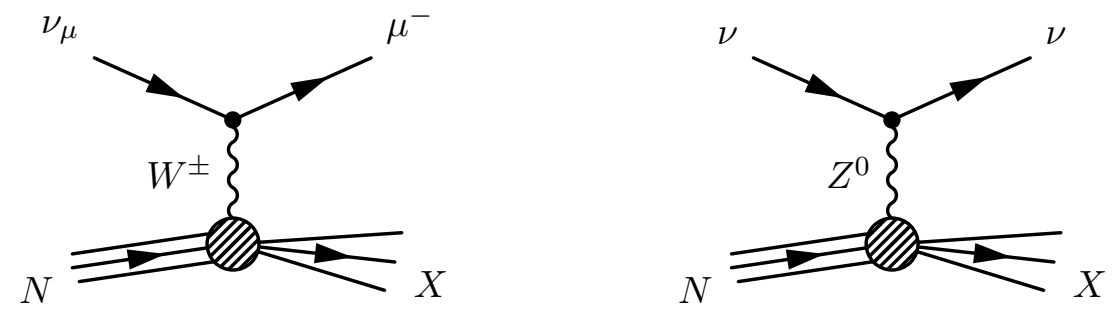

Figure 1.1: Left: Charged-current neutrino-nucleon scattering. Right: Neutral-current neutrino-nucleon scattering.

neutrino scattering on a nucleon, $N$. The inclusive diagrams are shown. Utilizing the Lagrangians, assuming highly off-shell vector bosons, and no charm or heavier quark production in the intermediate or final states, the Feynman rules [11] dictate the transition amplitudes:

$$
\begin{aligned}
T_{C C}\left(\nu_{\mu} N \rightarrow \mu^{-} X\right) & =\frac{g^{2} \cos \theta_{c}}{2 M_{W}^{2}}\left[\bar{e}_{\mu L} \gamma^{\alpha} \nu_{\mu L}\right]\left\langle X\left|J_{\alpha}\right| N\right\rangle \\
T_{N C}(\nu N \rightarrow \nu X) & =\frac{g^{2}}{2 M_{Z}^{2} \cos ^{2} \theta_{W}}\left[\bar{\nu}_{L} \gamma^{\alpha} \nu_{L}\right]\left\langle X\left|J_{\alpha}\right| N\right\rangle,
\end{aligned}
$$

where the leptonic current is exactly calculable (square brackets), and the hadronic current $\left\langle X\left|J_{\alpha}\right| N\right\rangle$ is parametrized by a model for a chosen final state $X$. The neutrinos, being chargeless, are able to penetrate deep into nucleons. The experimental measurements, coupled with theoretical understanding, yield insight into the structure of nucleons.

The current understanding of CC inclusive neutrino-nucleon interactions is summarized in Fig. 1.2. Interactions of high-energy neutrinos, $E_{\nu}>10 \mathrm{GeV}$, are dominated by deep inelastic scattering (DIS), which obliterates the nucleon and/or nucleus. The low energies are an amalgamation of DIS, single pion production, and quasi-elastic scattering $(\nu n \rightarrow l p)$. At energies above $100 \mathrm{GeV}$ the measurements are extremely precise. 


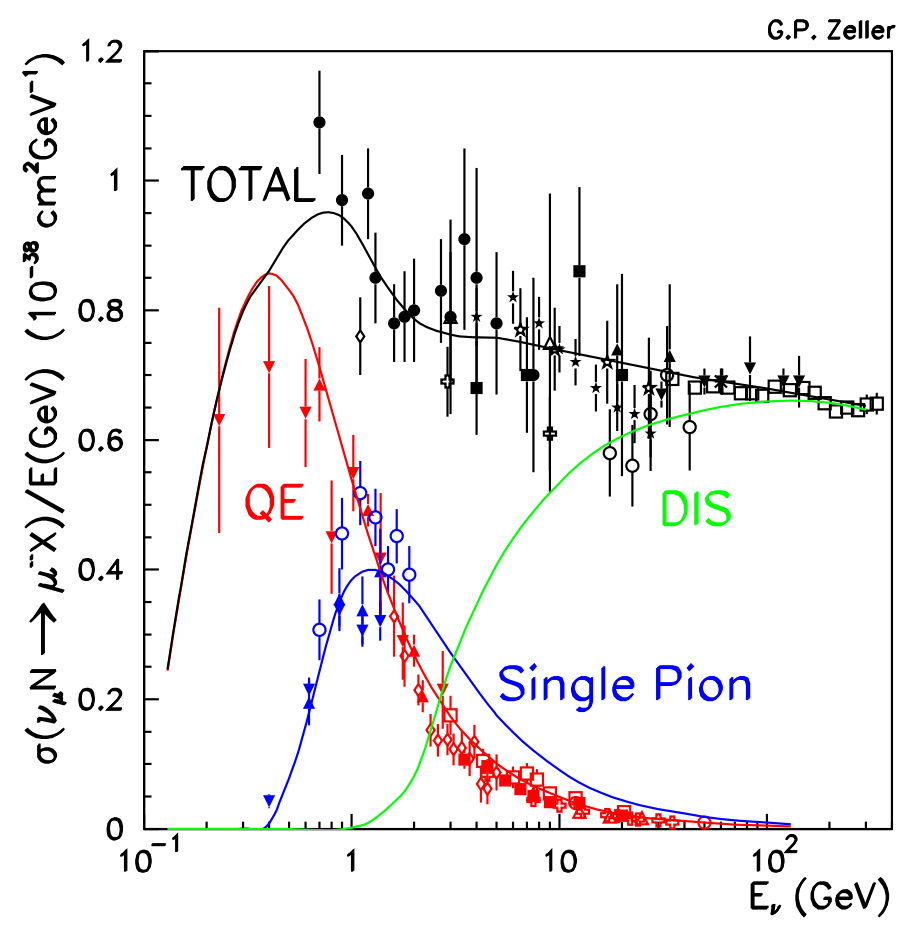

Figure 1.2: World data for charged-current (CC) neutrino interaction cross-sections [12].

In the range of a few hundred $\mathrm{MeV}$ to tens of $\mathrm{GeV}$ the measurements can be improved.

\subsubsection{Standard Model Extensions}

The SM is not complete with regard to neutrinos. For decades there was a problem that the number of measured neutrinos coming from the sun was inconsistent with the prediction from the standard solar model $[4,5,6]$. The resolution to this problem, neutrino oscillations $[13,14]$, has led to many interesting consequences. The first is that neutrinos have mass, implying that they must have a right-handed component. The second is that the mass states differ from the neutrino flavor states which allows for the flavor states to oscillate as a neutrino propagates. The third is that $\mathrm{CP}$ violation, previously observed in the quark sector, may occur in the leptons. The fourth is that neutrinos may be their own anti-particles. 
The invisible decay width of the $Z^{0}$ boson shows that there are only three light neutrinos that participate in the weak interaction [15]. This does not rule out higher mass neutrinos that may couple to the $Z^{0}$, nor does it rule out light neutrinos without couplings (so called "sterile" neutrinos).

\subsubsection{Oscillations}

Neutrino oscillations $[16,17,18]$ effectively solved the solar neutrino deficit problem. The basic physics of neutrino oscillations stems from the understanding that neutrino flavor states, the states that participate in the weak interaction, are superpositions of the neutrino mass states. This implies that a neutrino created in one flavor will propagate as a superposition of mass states and will interact some distance away as a mixture of the possible flavor states. However, that just described neutrino mixing; oscillations occur because there is a time dependence to the mixing that is proportional to the neutrino energy. This means that neutrinos of different energies mix at different rates for the same time or distance. The probability that a neutrino of flavor $\alpha$ oscillates to flavor $\beta$ is

$$
\begin{aligned}
& \mathcal{P}(\alpha \rightarrow \beta)=\delta_{\alpha \beta}-4 \sum_{i>j} \mathfrak{R e}\left(U_{\alpha i}^{*} U_{\alpha j} U_{\beta j}^{*} U_{\beta i}\right) \sin ^{2}\left(\frac{\Delta m_{i j}^{2} L}{4 E}\right) \\
& \pm 2 \sum_{i>j} \mathfrak{I m}\left(U_{\alpha i}^{*} U_{\alpha j} U_{\beta j}^{*} U_{\beta i}\right) \sin \left(\frac{\Delta m_{i j}^{2} L}{2 E}\right),
\end{aligned}
$$

where $i, j$ are the mass state indices, $\mathbf{U}$ is the lepton mixing matrix, $\Delta m_{i j}^{2} \equiv m_{i}^{2}-m_{j}^{2}$, $L$ is the distance traveled in the lab frame, $E$ is the neutrino's energy, and ' + ' is for neutrinos and '-' is for anti-neutrinos.

\subsubsection{Light Right-handed Neutrinos}

The fact neutrinos have mass means that a left-handed neutrino does not travel at the speed of light. This implies that one could boost to a frame along the neutrino 
direction where the neutrino's momentum flips. Since the spin is unaffected by the boost, the neutrino is then right-handed in that frame.

\subsubsection{CP violation}

The possibility of $\mathrm{CP}$ violation in the lepton sector is tantalizing because the amount of known $\mathrm{CP}$ violation in the quark sector is not enough to explain the matter anti-matter asymmetry in the universe [19]. Additionally, CP violation only occurs in $\mathrm{CC}$ interactions; the matrix element exactly cancels in NC interactions. Whether even maximal $\mathrm{CP}$ violation in the lepton sector would solve this problem is unclear.

The lepton mixing matrix, $\mathbf{U}$, is unitary. A unitary matrix is parametrized in general by $n(n-1) / 2$ angles and $n(n+1) / 2$ complex phases where $n$ is the dimensionality; for leptons $n=3$ and there are 3 angles and 6 phases. Some of the phases can be absorbed into the definitions of the particle wave functions. As the unitary matrix is unchanged if all the particles are changed by the same phase, $2 n-1$ phases can be absorbed by the particle wave functions. With three flavors, this leaves only one phase. The consequence is that the coupling of leptons vs. anti-leptons can be different from each other in $\mathrm{CC}$ weak interactions, hence $\mathrm{CP}$ violation. However, this is subject to a few conditions: the masses must not be degenerate, and the mixing matrix must have three non-zero angles (i.e. there is no parametrization that describes the mixing with only two angles). If these conditions are met, then it is possible for there to be a non-trivial complex phase and $\mathrm{CP}$ violation.

Two of the mixing angles are known to be large, $\theta_{23} \geq 38^{\circ}$ and $\theta_{12} \simeq 34^{\circ}$ [20]. The third is currently limited to $\theta_{13}<13^{\circ}$ [21]. If the remaining mixing angle is too small, $\theta_{13} \sim 0$, then there would be no $\mathrm{CP}$ violation in $\mathrm{CC}$ lepton interactions. 


\subsubsection{Majorana Neutrinos}

The possibility exists that neutrinos may be their own anti-particles (these are called Majorana neutrinos). Dirac mass terms in the Lagrangian require both rightand left-handed components of a particle. However, an additional mass term can be constructed from single right-or left-handed neutrinos through charge conjugation. This results in an anti-neutrino of the same chirality, or handedness, and a term can be added to the Lagrangian that performs this conjugation. Effectively this adds mass to the neutrino, and also allows for the transitions $\nu \leftrightarrow \bar{\nu}$; the neutrino is its own anti-particle. As neutrinos are highly relativistic, chirality is a relatively good quantum number. Since the weak interaction only affects left-handed neutrinos and right-handed anti-neutrinos, the rate of transitions is suppressed by $O(m / E)$.

\section{$1.2 \quad \mathrm{CC} \pi^{0}$ Interactions}

As neutrino oscillation experiments get more precise over the next decade, they will need accurate predictions of their event rates for both signals and backgrounds. A common mode for searching for neutrino oscillations is the charged-current quasi-elastic $(\mathrm{CCQE})$ interaction $\left(\nu n \rightarrow l^{-} p\right)$. The CCQE interaction is used at energies less than a few $\mathrm{GeV}$ because they have both a simple final state, which makes a measurement of the neutrino energy easier, and they dominate the event rate. As neutrino oscillations are only dependent on whether a CC neutrino interaction took place, any CC mode can be used to search for oscillations.

For a CCQE neutrino oscillation search, one of the backgrounds is CC single $\pi^{0}$ $\left(\mathrm{CC} \pi^{0}\right)$ production $\left(\nu_{\mu} n \rightarrow \mu^{-} \pi^{0} p\right)$. While $\mathrm{CC} \pi^{0}$ events comprise a small fraction of the total neutrino interaction rate, they tend to end up in CCQE selection samples. This leads to misreconstructed CCQE events and an overall smearing of the CCQE reconstructed neutrino energy (the only relevant parameter in oscillations). The $\mathrm{CC} \pi^{0}$ 
events either have to be accurately predicted, or measured directly and subtracted from the CCQE sample.

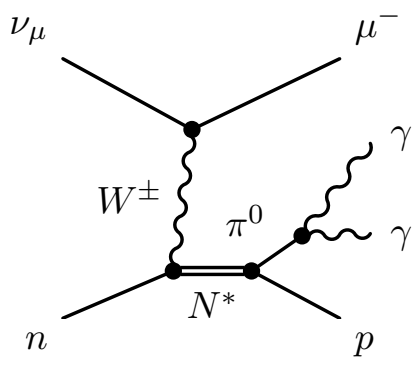

Figure 1.3: A tree level $\mathrm{CC} \pi^{0}$ Feynman diagram propagated through a nucleon resonance, $N^{*}$.

All CC and NC single pion production interactions, without the production of additional mesons, have a coherent contribution due to neutrino-nucleus (opposed to nucleon) scattering except, for $\mathrm{CC} \pi^{0}$ interactions (See Fig. 1.3). These interactions can only occur through a nucleon resonance. Studying $\mathrm{CC} \pi^{0}$ interactions, along with additional single pion modes, may garner insight into both the resonant and coherent contributions to neutrino-induced single pion production.

\subsection{Dissertation Outline}

This dissertation details a measurement of $\nu_{\mu}$-induced CC single $\pi^{0}\left(\mathrm{CC} \pi^{0}\right)$ production on a $\mathrm{CH}_{2}$ target. The motivations for doing so are that the current experimental knowledge of this mode is incomplete, and that as oscillation experiments become precise they will need better estimation of rare mode backgrounds. In Chapter 2 the MiniBooNE experiment is introduced and fully detailed. Chapter 3 describes a side project that was designed to constrain backgrounds for the neutrino oscillation measurement. Chapter 4 describes the method of reconstructing $\mathrm{CC} \pi^{0}$ interactions. Finally, the extraction of the total inclusive cross-section along with several differential cross-sections are presented in Chapter 5. 


\section{Chapter 2}

\section{MiniBooNE}

The Mini Booster Neutrino Experiment (MiniBooNE) [22] at Fermilab was commissioned to address the LSND anomaly. The LSND experiment at Los Alamos measured an unexpected neutrino oscillation signature with a mass splitting on the order of $\sim 1 \mathrm{eV}^{2} / c^{4}[23,24,25,26]$. Since the other two mass splittings are on the order of $\sim 10^{-3} \mathrm{eV}^{2} / c^{4}$ (atmospheric) and $\sim 10^{-4} \mathrm{eV}^{2} / c^{4}$ (solar), the LSND mass splitting disagrees with a three neutrino scenario. MiniBooNE performed both appearance [27, 28] and disappearance [29] searches and saw no evidence for the LSND signal. Since that time, MiniBooNE has been working on further oscillation studies and neutrino crosssection measurements.

\subsection{Experimental Apparatus}

The MiniBooNE experiment takes protons from the Fermilab Booster and impinges them on a beryllium target housed in a magnetic focusing horn with a toroidal field. The resulting charged pions and kaons are focused/de-focused according to their charge. The focused meson beam decays in flight producing a predominantly $\nu_{\mu}$ beam that travels half a kilometer through dirt until interacting with an 800 ton mineral oil neutrino detector. The interactions of neutrinos with the mineral oil produce particles whose interactions with the oil produce photons that are detected by photo-multiplier tubes (PMTs) along the detector's wall. Fig. 2.1 shows a diagram of the MiniBooNE 


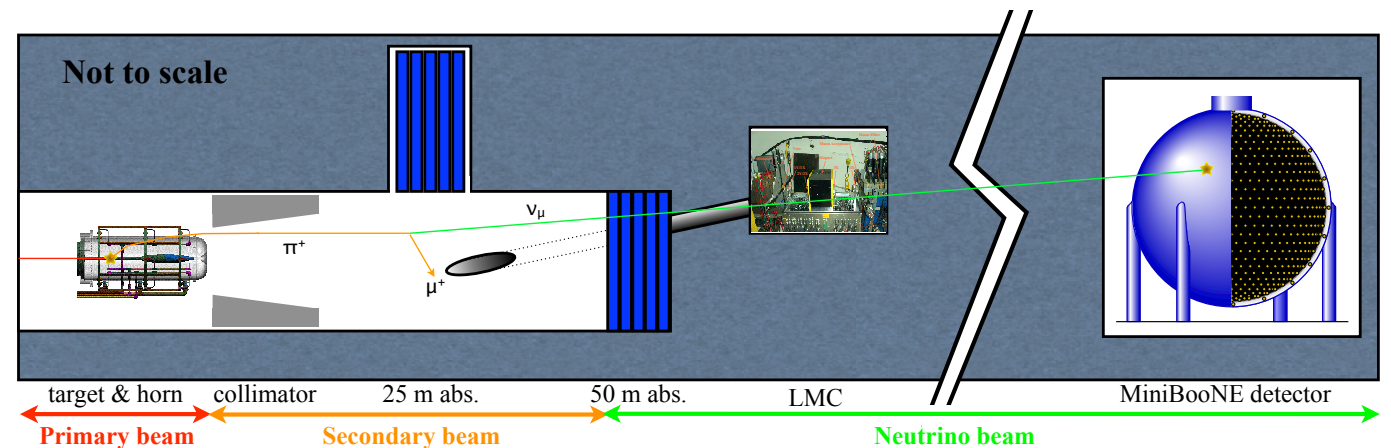

Figure 2.1: A cartoon of the MiniBooNE experiment. Pions and kaons are produced by interactions of protons on the beryllium target embedded in a magnetic focusing horn. The horn creates a toroidal magnetic field that focuses positively charged particles into the $50 \mathrm{~m}$ decay region. The particles decay-in-flight producing a forward neutrino beam. The beam passes through $500 \mathrm{~m}$ of earth before coming into contact with the MiniBooNE detector. Off-axis the secondary beam is a drift pipe that leads to the LMC enclosure (see Chapter 3).

experiment.

\subsubsection{Primary Beamline}

The MiniBooNE primary beam comes from the Fermilab booster [30]. The booster is the third accelerator in the Fermilab chain of accelerators. The first accelerator is a Cockcroft-Walton DC accelerator that accelerates $\mathrm{H}^{-}$ions to $750 \mathrm{keV}$. The second accelerator in the chain is a linear accelerator, called the Linac, that accelerates the $\mathrm{H}^{-}$ions to $400 \mathrm{MeV}$ through a series of $\mathrm{RF}$ cavities. The beam passes through a foil to strip the 2 electrons from the ions before entering the booster. Once in the booster the protons get accelerated to a kinetic energy of $8 \mathrm{GeV}$.

\subsubsection{Booster and BNB}

The booster is a $474 \mathrm{~m}$ circumference synchrotron accelerator. The Linac pulse occurs over $20 \mu \mathrm{s}$, or ten revolutions of the beam in the booster. The beam is bunched 
into packets 2 ns wide and the packets are 18.9352 ns apart. The harmonic number of the booster is 84 , though there are typically only 81 bunches. Protons are accelerated to $8 \mathrm{GeV}$ in $0.033 \mathrm{~s}$ over 16,000 cycles around the booster. The operational frequency is $15 \mathrm{~Hz}$, determined by the resonant capacitive circuit needed to balance the inductance of the magnets. The protons are then transferred toward the Main Injector with an additional transfer off the the main injector line into the Booster Neutrino Beamline (BNB) [31]. The transfers occur for up to 11 consecutive cycles for an average rate of 5 $\mathrm{Hz}$ with $5 \times 10^{12}$ protons per pulse. Not every Booster cycle gets used for MiniBooNE and typically the beamline received between $3-4 \mathrm{~Hz}$ for normal operations. Fig. 2.2 shows the spatial layout of the BNB.

The BNB is comprised of a lattice of focusing/de-focusing (FODO) magnets to keep the beam emittance low, and several dipole magnets to steer the beam into the MiniBooNE target. Fig. 2.3 shows the optics of the BNB. Several beam position monitors $(\mathrm{BPM})$ track the position of the beam to ensure that the proton beam impacts the target within acceptable tolerances. Fig. 2.4 shows the weekly protons on target (p.o.t.) delivered over the course of the MiniBooNE run periods.

\subsubsection{Target and Horn}

The MI-12 surface building houses the target, horn, and power supplies for the horn. Fig. 2.5 shows a schematic of the target pile. The target, horn, collimator, and shielding blocks are detailed. Additionally, the horn and target cooling systems are housed in this building.

The protons in the BNB end their journey with an impact into a beryllium target that is embedded in a magnetic focusing horn. The target is constructed from beryllium, chosen because of its low $Z$ and fast radioactive cool down time in the event of replacement. The target is constructed of 7 cylindrical "slugs" $10.2 \mathrm{~cm}$ in length, and $0.48 \mathrm{~cm}$ in radius. The slugs are placed end to end forming the $71.1 \mathrm{~cm}$ target length, 


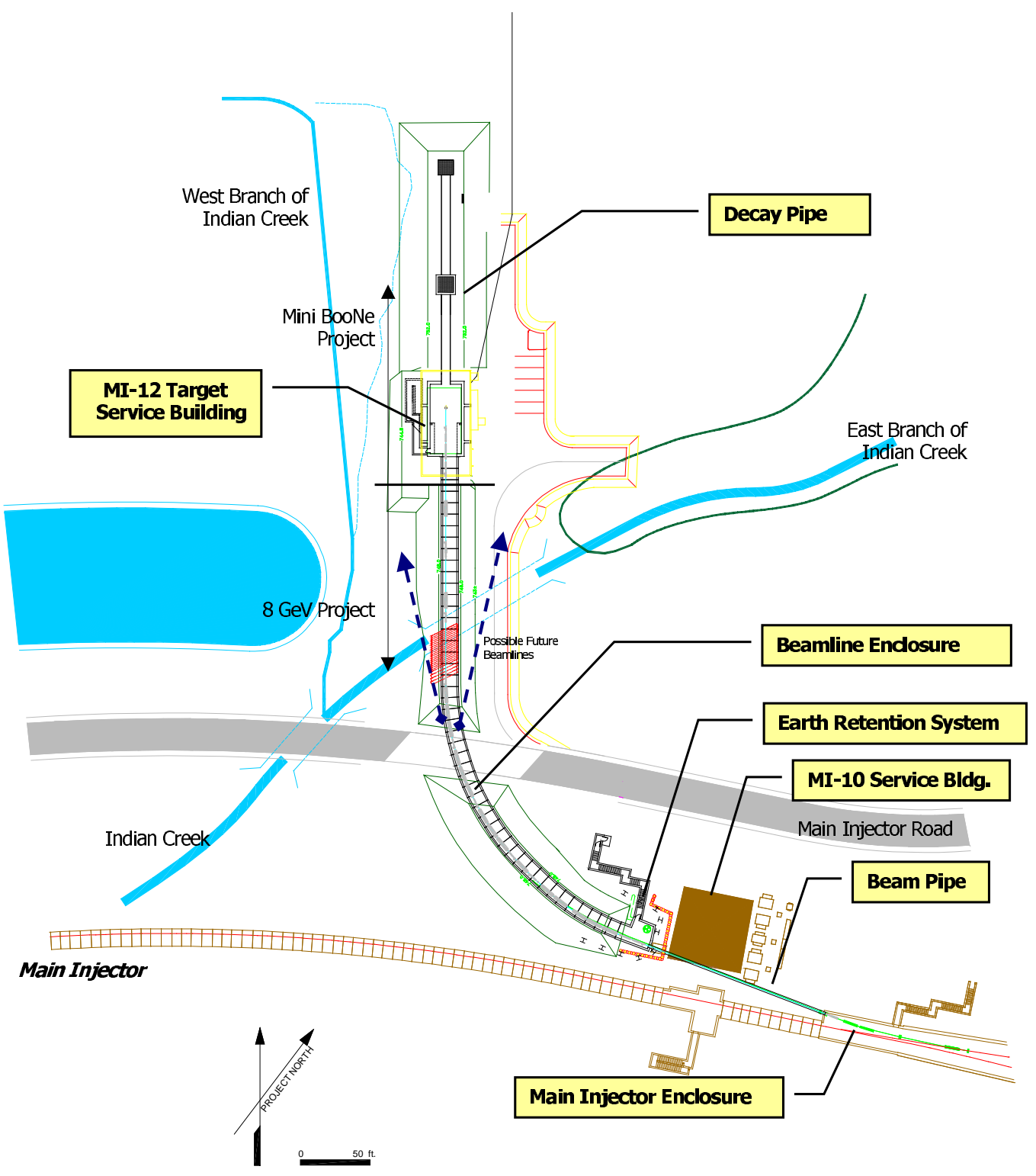

Figure 2.2: Drawing of the $8 \mathrm{GeV}$ transfer line off the Main Injector, the MiniBooNE primary beamline, target hall, and decay region. Schematic taken from Ref. [31].

which is 1.7 proton interaction lengths. Attached in intervals of $120^{\circ}$ are three beryllium fins, supporting each slug within a beryllium tube. The tube is $0.9 \mathrm{~cm}$ thick with an 


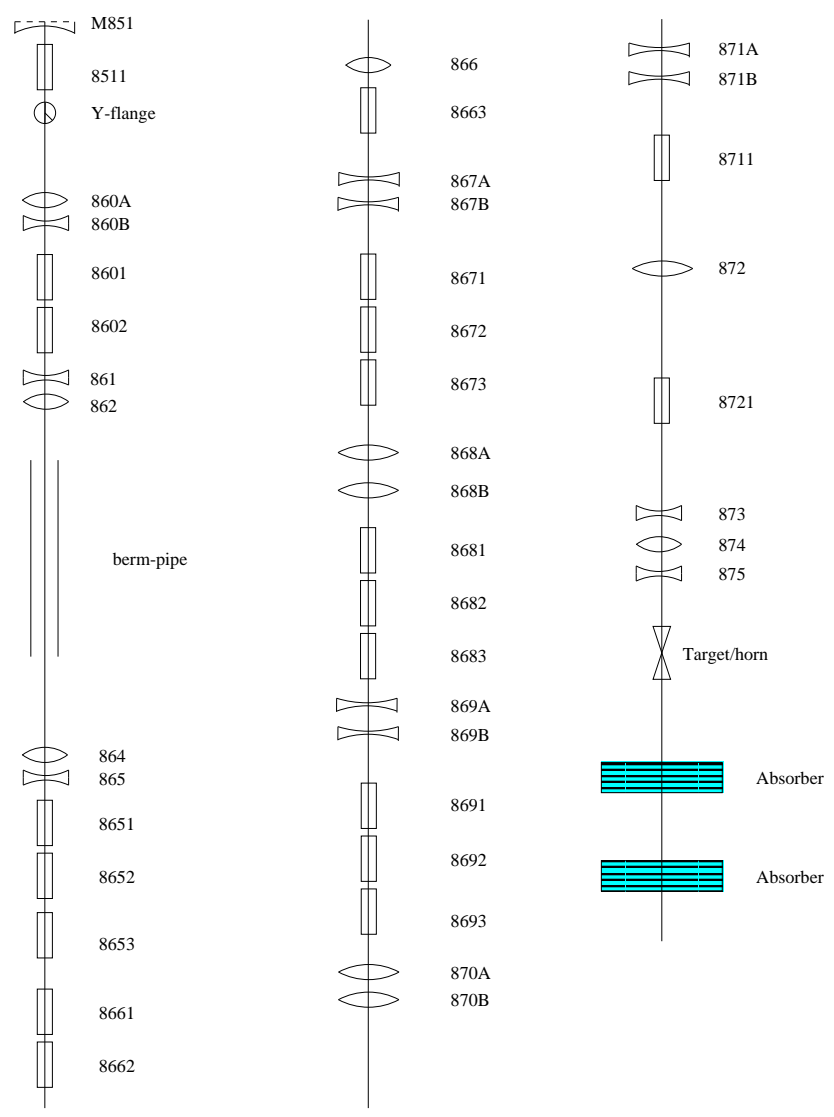

Figure 2.3: Detail of the magnets in the $8 \mathrm{GeV}$ transfer line. The primary proton beam is transferred to the line through a kicker magnet at the top left. The beam passes through a series of focusing, de-focusing, and dipole magnets until it reaches the MI12 surface building and the target. Protons that pass through the target impact an absorber 25 or $50 \mathrm{~m}$ downstream. Schematic taken from Ref. [31].

inner radius of $1.37 \mathrm{~cm}$. The full target structure is cantilevered from the upstream end within the center of the horn. The target is also completely electrically isolated from the horn. Fig. 2.6 shows the target assembly. During normal operations the target must be cooled from the $\sim 600 \mathrm{~W}$ of power deposited by the proton beam. The target is cooled 


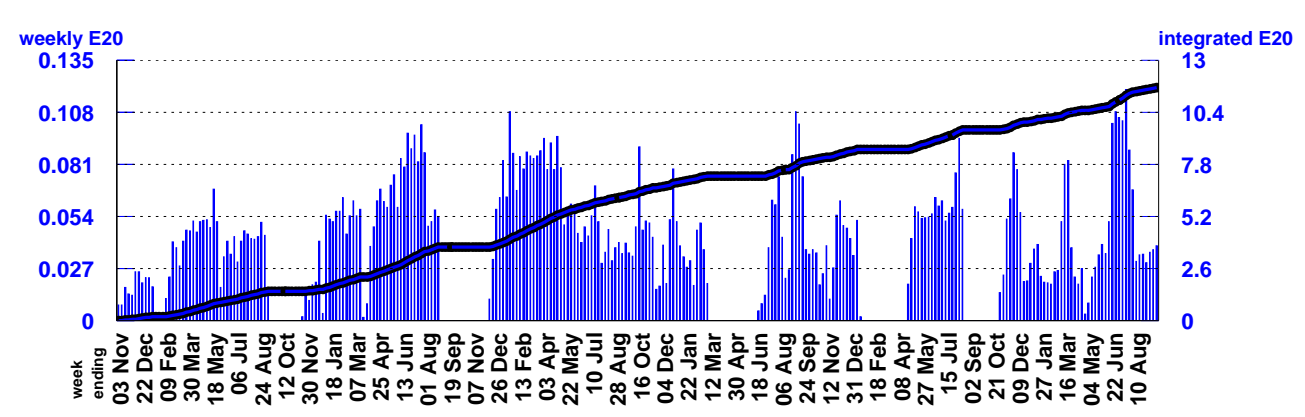

Figure 2.4: The weekly number of protons delivered to the target from November 2002 through August 2008. Data in neutrino mode were taken 2/02/2003-1/19/2006 and $10 / 14 / 2007-4 / 08 / 2008$ constituting $6.46 \times 10^{20}$ p.o.t. in neutrino mode.

by circulated air, transferring the heat through a series of heat exchangers to contain the radioactive air. The temperature and radiation levels of the air are continuously monitored. If any abnormalities are detected, the proton beam is automatically shut off.

The target is embedded within a magnetic focusing horn. Current, brought into the target pile through striplines, runs along the length of the inner conductor, and returns over the outer conductor creating a toroidal magnetic field between the conductors. The horn is pulsed by a half-sinusoidal current over $143 \mu$ s to a peak at 170 $\mathrm{kA}$. The current is effectively constant during the $1.6 \mu$ s long beam spill. Ampere's law dictates a field,

$$
B_{\phi}(r)=\frac{\mu_{0} I}{2 \pi r}
$$

between the conductors where $\mu_{0}$ is the permeability of free space, $I$ is the current along the inner conductor, and $r$ is the radius from the center of the target. The toroidal field focuses positively charged particles, and de-focuses negatively charges ones. The extreme currents pulsed through the horn cause it to heat up through ohmic heating. To cool the horn, a series of ports in the outer conductor are outfitted with water nozzles. These nozzles spray water directly onto the inner conductor. The horn has a series of drainage ports on the bottom side. The radioactive water is contained in a closed 


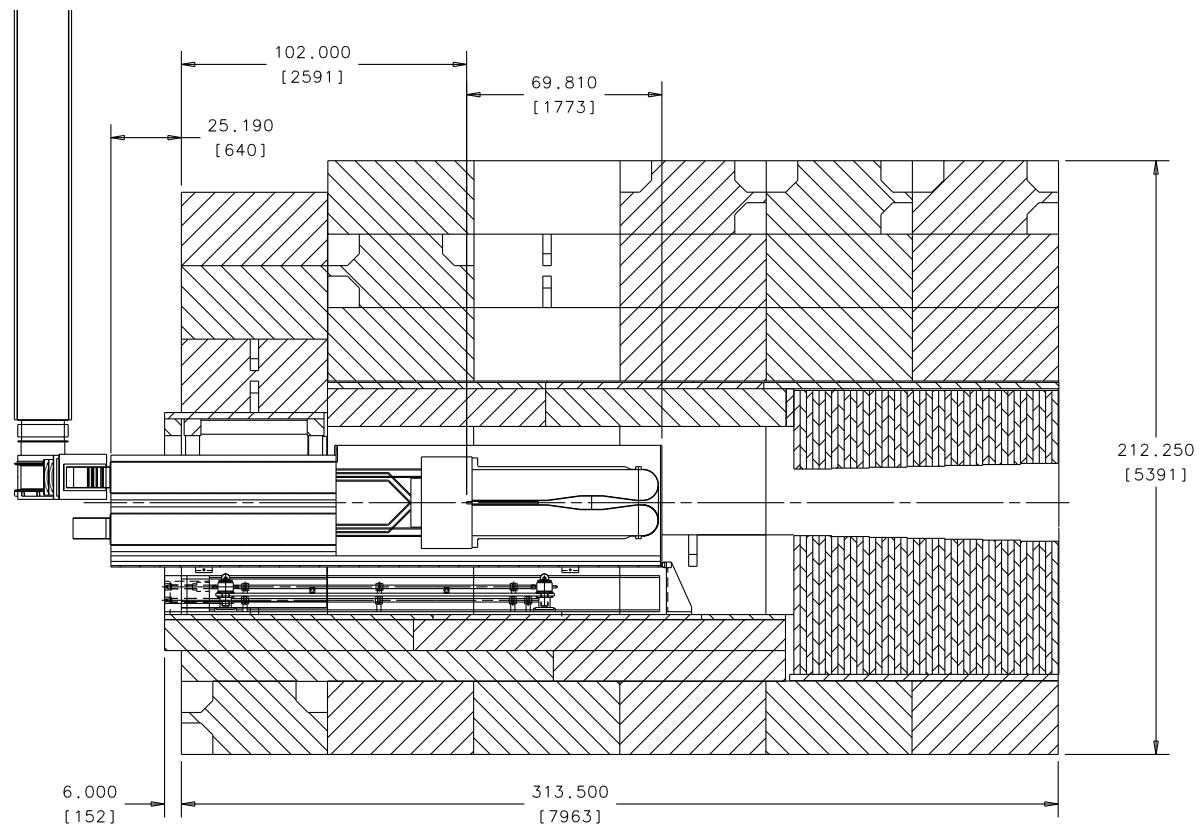

Figure 2.5: A schematic of the target pile. The protons entered from the left. Schematic taken from Ref. [32].

system. Fig. 2.7 shows a rendering of the magnetic horn with the attached cooling system. The horn undergoes large vibrations during the pulse, so the cooling system is not rigidly fixed and is attached by baffles to mitigate the transfer of the vibrations to the water system. 

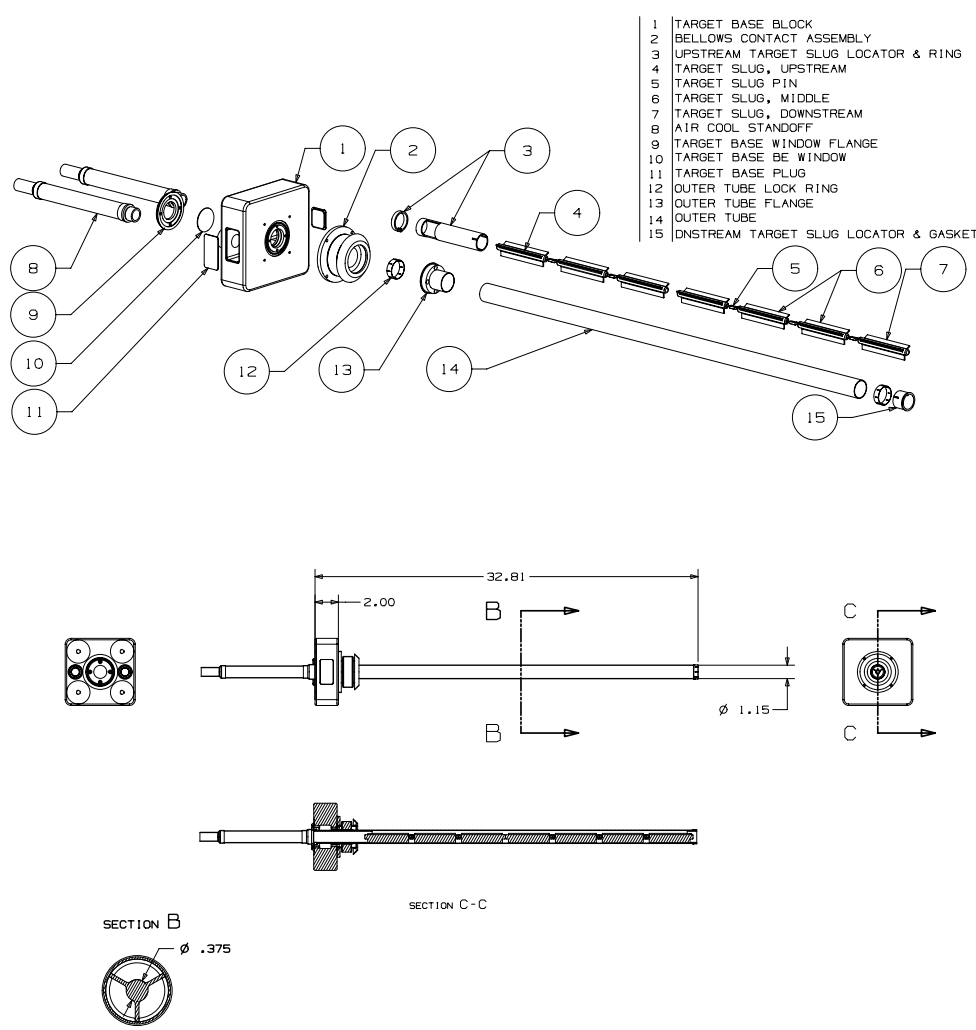

Figure 2.6: The beryllium target assembly. The target consists of 7 beryllium slugs for a total length of $71.1 \mathrm{~cm}$. Schematic taken from Ref. [33].

\subsubsection{Meson Decay Volume}

Immediately downstream from the horn and target is a steel collimator. The collimator is $214 \mathrm{~cm}$ long, and is located $259 \mathrm{~cm}$ from the front face of the target. The aperture is $60 \mathrm{~cm}$ in diameter, widening to $71 \mathrm{~cm}$ at the downstream end. The collimator begins the meson decay region, which extends $45 \mathrm{~m}$ beyond the collimator. The decay pipe is a circular, corrugated steel pipe, with a $183 \mathrm{~cm}$ radius. The dirt surrounding the pipe is composed of dolomite $\left(\mathrm{CaMg}\left(\mathrm{CO}_{2}\right)_{3} ; \rho=2.24 \mathrm{~g} / \mathrm{cm}^{3}\right)$. The end of the pipe (50m from the target) is a steel-and-concrete beam dump. The entire volume is filled with air at normal temperatures and pressures. 


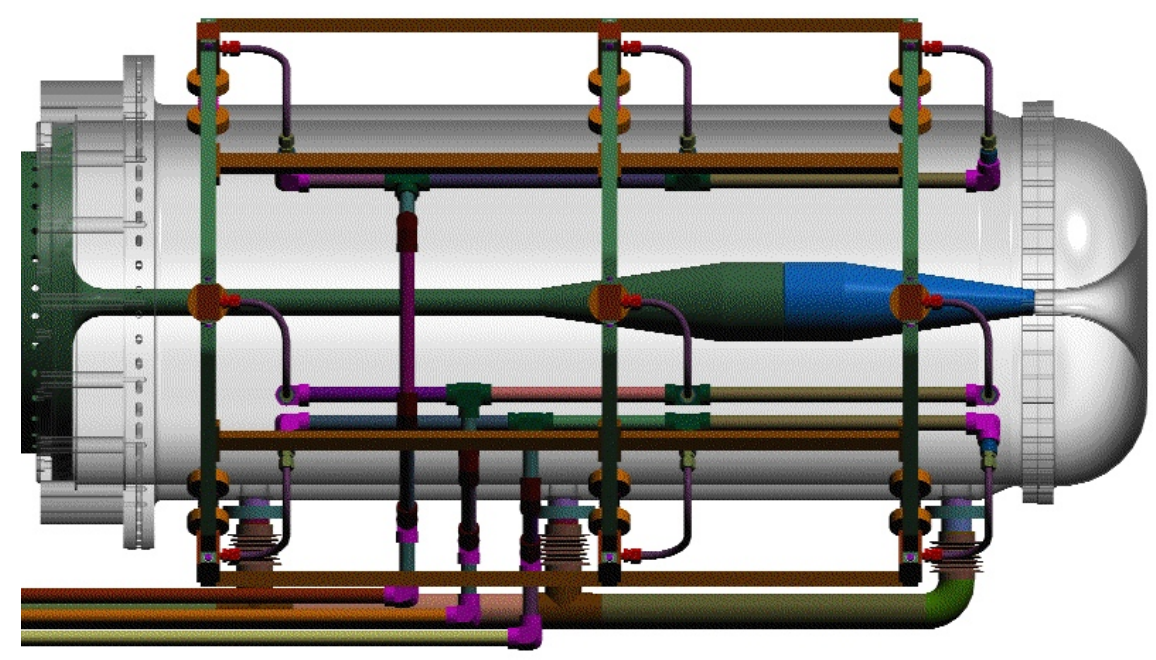

Figure 2.7: The magnetic horn assembly shown with the cooling apparatus. The outer conductor is shown semi-transparent to see the inner conductor. The proton beam enters from the left. Schematic taken from Ref. [32], courtesy of Bartoszek Engineering [34].

The length of the decay volume, while determining the $\nu_{\mu}$ from $\pi^{+}$decays in flight, allows for intrinsic $\nu_{e}$ from $\mu^{+}$decays. An additional steel absorber, suspended above the beam at $25 \mathrm{~m}$ downstream from the target, can be inserted to assess the systematic error associated with this source of intrinsic $\nu_{e}$. However, this study has not been performed. The location of the $25 \mathrm{~m}$ absorber adds a square enclosure near the middle of the decay pipe. At $41 \mathrm{~m}$ from the target connects an 8 inch drift pipe to the decay volume. This pipe, at a $7^{\circ}$ angle, points back into the $25 \mathrm{~m}$ absorber enclosure. Near the end of the drift pipe is a muon spectrometer that is discussed in detail in Chapter 3 .

\subsubsection{MiniBooNE Detector}

The MiniBooNE detector, located $541 \mathrm{~m}$ from the target (center to center) and centered $1.896 \mathrm{~m}$ above the axis of the neutrino beam, is a spherical tank $610.6 \mathrm{~cm}$ 
in radius filled with 818 tons of mineral oil. The detector has an opaque barrier 35 $\mathrm{cm}$ interior to the outer detector wall, $574.5 \mathrm{~cm}$ in radius from the center of the tank. The inner region is instrumented with 1280 inward facing 8 inch PMTs accounting for an $11.3 \%$ photo-cathode coverage area. The inner shell is painted black to reduce reflections. The outer region is instrumented by 240 sideways-facing 8-inch PMTs. These PMTs are mounted in back-to-back pairs. This region is painted white to increase reflections. The inner region confines the fiducial volume; the outer defines the veto for tagging entering or exiting events. Fig. 2.8 shows a schematic of the MiniBooNE neutrino detector.

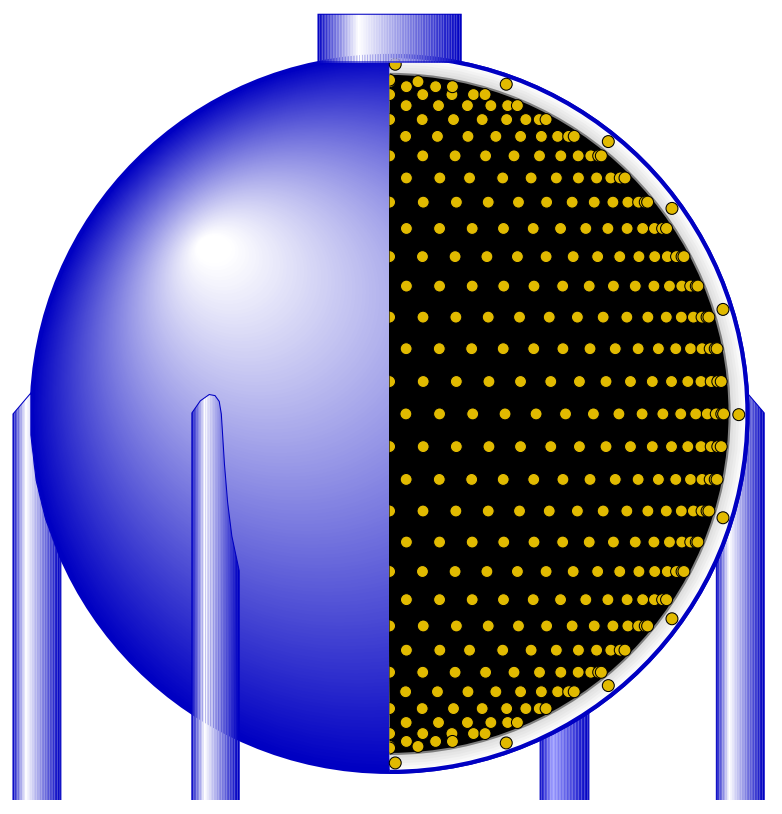

Figure 2.8: The MiniBooNE detector schematic shown with a quarter sphere cut out. The PMTs are displayed as the yellow dots. The optically isolated veto region is shown in white. Schematic taken from Ref. [35].

The MiniBooNE detector is partially underground to help reduce the ambient 
cosmic-ray background, and to put the detector more in line with the incident neutrino beam. Fig. 2.9 shows the layout of the detector enclosure. The electronics sit in a room

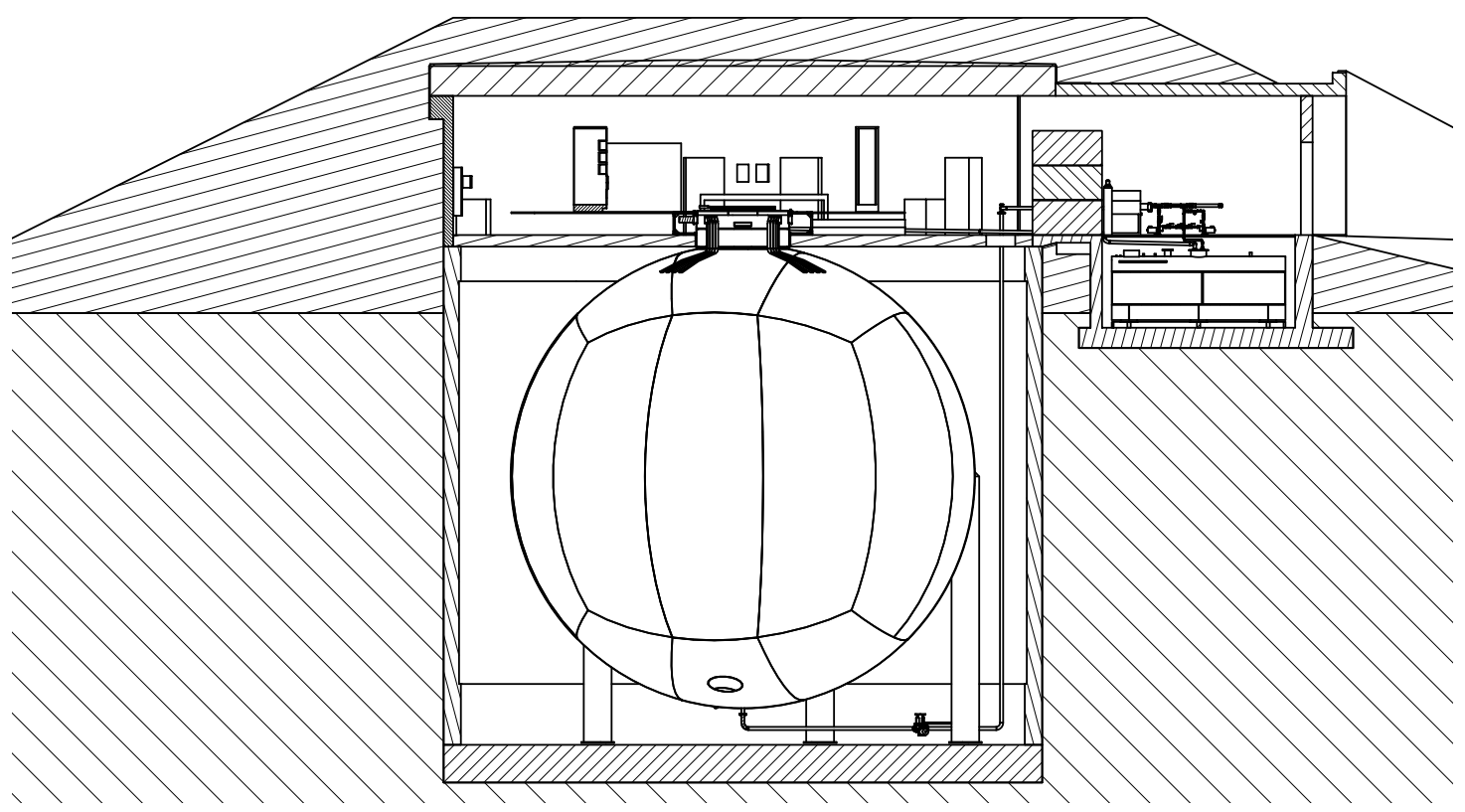

Figure 2.9: The MiniBooNE detector enclosure. The electronics plant sits above the detector. The oil plumbing system and overflow tank are on the right near the entrance to the enclosure. The slight dirt over-burden is apparent. Schematic taken from Ref. [35].

above the detector. Approximately $3 \mathrm{~m}$ of dirt and concrete sit above the enclosure, reducing the cosmic-ray rate to below $10 \mathrm{kHz}$. Additionally, a 2500 gallon capacity tank handles overflows of the mineral oil in the event of thermal expansion.

MiniBooNE uses two types of PMTs: 1198 Hamamatsu [36] R1408 and 322 Hamamatsu R5912 PMTs. The R1408 tubes were inherited from the LSND experiment and the R5912 were purchased specifically for MiniBooNE. As the R5912 tubes had better timing and charge resolutions than the R1408 [37], all R5912 were used in the main tank. The veto tubes were chosen from the R1408 tubes with the lowest dark noise. 


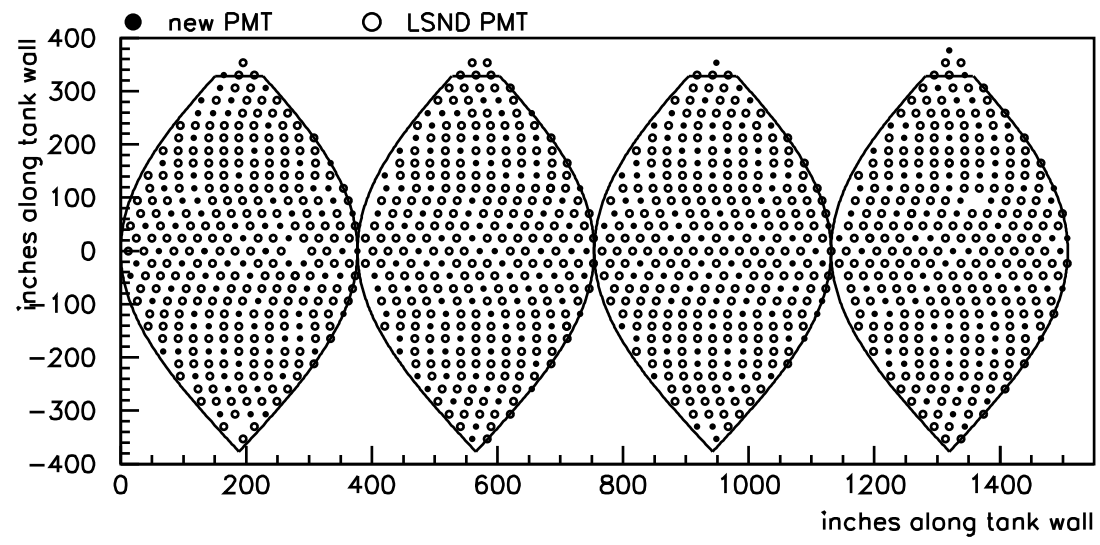

Figure 2.10: The mapping of the placement of the new (R5912) and old (R1408) PMTs within the detector. Schematic taken from Ref. [35].

Fig. 2.10 shows the relative positions of the tubes in the main tank. Both types have a high gain of $1.6 \times 10^{7}$ electrons per photo-electron $(\mathrm{PE})$. A schematic showing the PMT mounting in both the main tank and the veto is shown in Fig. 2.11. Note, the inward facing main tank PMTs, and tangential facing veto tubes.

\subsubsection{Mineral oil}

The mineral oil was chosen from a sample of ten different mineral oils [38]. The criteria included a high index of refraction, small dispersion, and large attenuation length for wavelengths across the visible spectrum. The Exxon/Mobil Marcol 7 mineral oil was chosen as satisfying these criteria. The optical properties of the mineral oil 


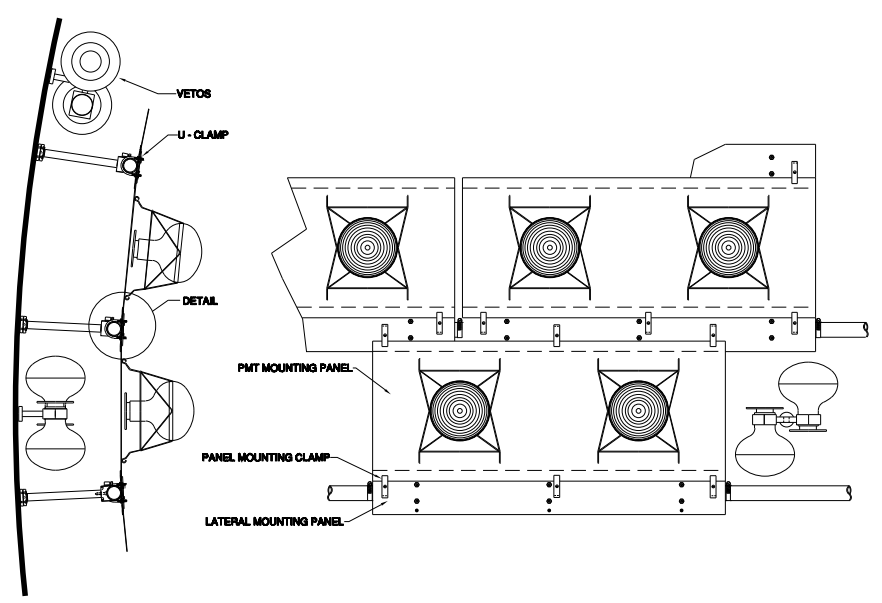

Figure 2.11: The mounting brackets and inner wall that the tank PMTs attach to. The back to back placement of the veto PMTs is also apparent. Schematic taken from Ref. [35].

will be discussed in $\S 2.2 .3 .2$. Marcol 7 has a density of $0.845 \pm 0.001 \mathrm{~g} / \mathrm{cm}^{3}$. Mineral oil has a lower $\mu^{-}$capture rate than water ( $8 \%$ vs $20 \%$ ), making muon tagging more efficient. The mineral oil can be continuously recirculated, though it was found to not be necessary. Fig. 2.12 shows the recirculation schematic. The oil is extracted at the top of the tank, and recirculated into the bottom after passing through the overflow tank.

\subsubsection{Detector Electronics}

Recording the full PMT pulses for 1520 channels for each trigger is extremely impractical. Instead, the total deposited charge and the time that a pulse crosses the discriminator threshold $(\sim 0.25 \mathrm{PE})$ are kept. The PMT pulses are amplified by a factor of 20 by preamps. As the pulses passed the discriminator threshold, the signals trigger an asynchronous discriminator producing a long triangular waveform, $V_{t}$, tagging the 


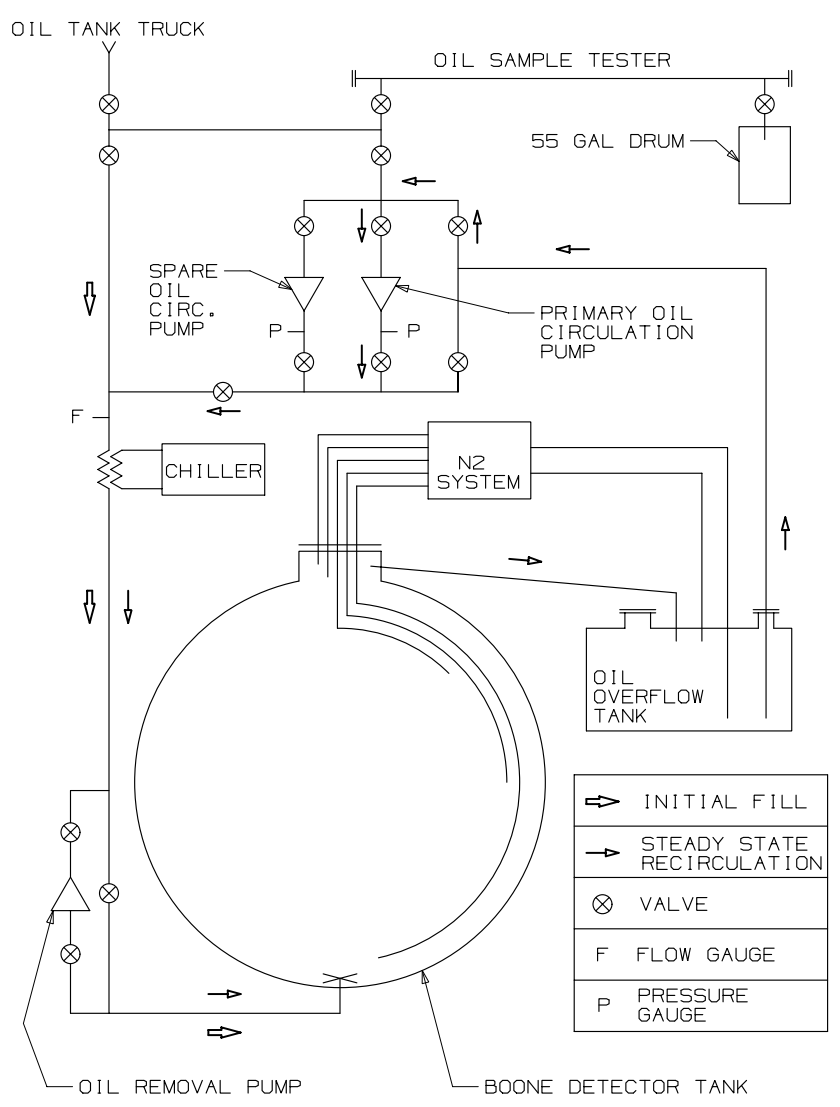

Figure 2.12: The oil circulation system. Schematic taken from Ref. [35].

pulse's start time. A capacitive integrator with a long time constant integrates the charge, $V_{q}$. Two 8-bit flash ADCs (FADCS) sampling continuously at $10 \mathrm{MHz}$ (every $100 \mathrm{~ns}$ ) record the waveforms at times $t-1$ through $t+2$, where $t$ is time of the synchronous discriminator, triggered by the asynchronous discriminator pulse. These samples are referred to as the time and charge "quad." Only two of the samples in the time quad, $t$ and $t+1$, are necessary to extrapolate the time. For the charge quad, $t-1$ is necessary to give the baseline, and times $t$ through $t+2$ are used to get the integrated charge as the capacitors' time constant is known. Fig. 2.13 shows a cartoon displaying 


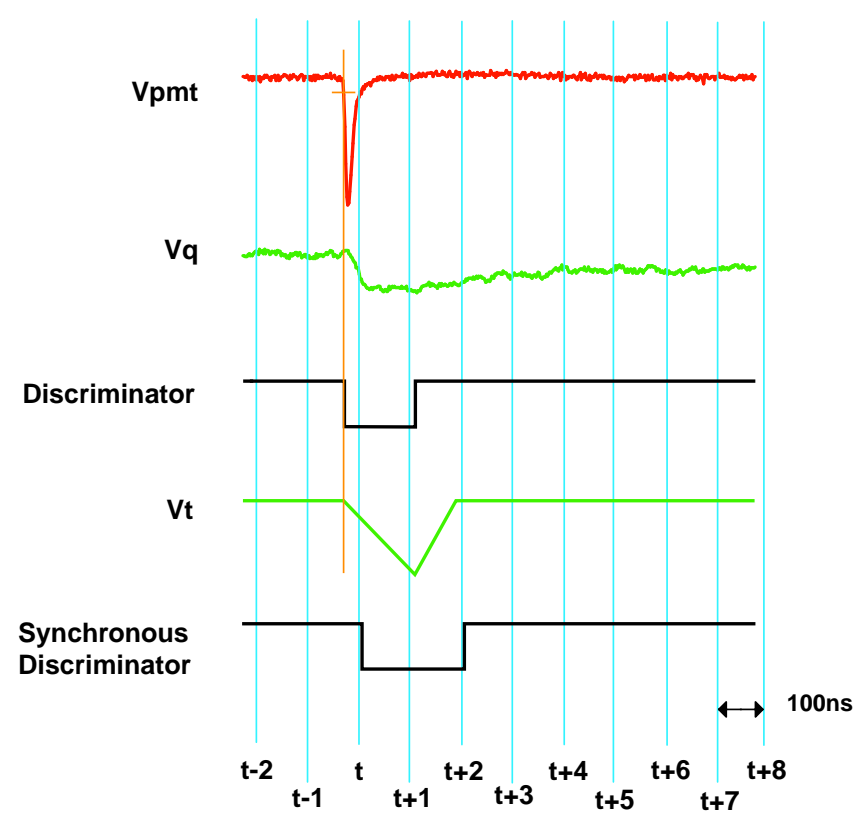

Figure 2.13: A PMT hit triggers an asynchronous discriminator that starts a voltage ramp, and immediately starts integrating the charge. The synchronous discriminator starts on the next clock tick after the trigger. Fast ADCs record the pulses $V_{t}$ and $V_{q}$ at 4 times, every $100 \mathrm{~ns}$, from $t-1$ through $t+2$. The total charge and initial time of the pulse are deduced from the FADC readout based on the fact that the shape of $V_{t}$ is fixed. Schematic taken from Ref. [35].

a PMT hit, $V_{P M T}$, the FADCs sampling times, and the corresponding time and charge quad information.

\subsubsection{Triggering}

The trigger conditions are based on external information provided from the Booster, internal calibration sources, and PMT multiplicities. PMT sum cards record the number of synchronous discriminator fires during any given clock tick separately for the tank, $N_{\text {tank }}$, and the veto, $N_{\text {veto }}$. As the overall activity in the detector is $\sim 10 \mathrm{kHz}$, the rates need to be prescaled to manage the activity. The event triggers are as follows: 
- Beam: Primary trigger activated by the Booster sending beam down the BNB.

- NuMI: Triggered by beam to main injector neutrino beamline. This does not overlap with the BNB.

- Strobe: A random trigger generated by a pulser at $2.01 \mathrm{~Hz}$.

- Laser: A laser calibration trigger (see $\S 2.1 .4 .4$ ).

- Cube: A cosmic event in calibration cube (see §2.1.4.4).

- Tracker: 4-fold coincidence in the cosmic-ray muon tracker hodoscope (see $\S 2.1 .4 .4)$.

- Michel: Stopped muon decay (Michel) trigger. Requires $N_{\text {tank }} \geq 200, N_{\text {veto }}<$ 6 currently to tag the electron and $N_{\text {tank }} \geq 100, N_{\text {veto }} \geq 6$ between 3 and $15 \mu \mathrm{s}$ prior to tag the muon.

- Supernova: Requires $N_{\text {tank }} \geq 60, N_{\text {veto }}<6$ and that the previous event was more than $15 \mu$ s prior.

- Veto: Activity trigger, requires $N_{\text {veto }} \geq 6$.

- Tank: Activity trigger, requires $N_{\text {tank }} \geq 60$.

- other- $\nu$ : Event consistent with a neutrino but not during the beam windows, requires $N_{\text {tank }} \geq 200, N_{\text {veto }}<6$.

Table 2.1 shows the trigger rates and prescales, if any. The events rates are scaled to $\sim 26 \mathrm{~Hz}$ of triggered activity. The accelerator-based triggers (beam and NuMI) are prioritized over all other triggers.

\subsubsection{Calibration}

The detector is calibrated through two in-situ sources: a pulsed laser and cosmicrays. The pulsed laser system injects a low level of light at $397 \mathrm{~nm}$ into the tank 
Table 2.1: The trigger rates, time windows, and prescales for each trigger type.

\begin{tabular}{l|crc} 
Trigger & Rate $(\mathrm{Hz})$ & Prescale & Time Window $(\mu \mathrm{s})$ \\
\hline \hline Beam & $2-5$ & 1 & 19.2 \\
NuMI & 0.5 & 1 & 19.2 \\
Strobe & 2.01 & 1 & 19.2 \\
Laser & 3.33 & 1 & 9.6 \\
Cube & 1.1 & 1 & 12.8 \\
Tracker & 0.7 & 170 & 12.8 \\
Michel & 1.2 & 600 & 19.2 \\
Supernova & 9.9 & 1 & 3.2 \\
Veto & 0.4 & 5000 & 19.2 \\
Tank & 0.4 & 90000 & 19.2 \\
Other- $\nu$ & 0.66 & 1 & 19.2 \\
\hline Total & $\sim 26$ & &
\end{tabular}

through one of either the $410 \mathrm{~cm}$ flasks, or a bare optical fiber. The flasks are filled with a diffusive medium, Ludox colloidal silica [39]. During normal operations the flask closest the center of the tank is used. The laser is pulsed to produce a low occupancy of $\sim 40$ PMTs hit during a given flash. The laser is pulsed at $3.33 \mathrm{~Hz}$, however, the laser is vetoed during a beam extraction timing pulse. The laser is used to calibrate the time offsets between the tubes, the gain calibration (tuned to $1 \mathrm{PE}$ ), and charge dependent time-slewing effects.

The second calibration source exploits the ambient cosmic-ray rate. A scintillating paddle hodoscope is positioned above the detector to record the incident angle and entry point for cosmic-rays entering the detector. This hodoscope is referred to as the "Tracker." Positioned at seven locations around the tank are $5 \mathrm{~cm}$ scintillator cubes, optically isolated from the mineral oil, read out through optical fibers. The system records events consistent with particles that pass through the tracker, and stop and decay in the cubes. Assuming minimum-ionizing particles, the energy of the muon is exactly known based on the measured path length. The cubes are positioned such that a range of 20-800 MeV stopping muons entering from above can be studied. The tracker 
and cube calibration system is used to tune the event reconstructions. The details of the laser and tracker systems can be found in Ref. [40].

\section{$2.2 \quad$ Simulations}

As the rate in the detector is determined by the flux and cross-sections for the interactions, the simulations are separated into two stages: flux simulations and detector simulations. The beam simulations model the primary beam including the interactions with the beryllium target, the focusing horn, the collimator, and secondary meson beam volume to produce a flux of neutrinos in the direction of the detector.

The detector model utilizes the flux with a neutrino interaction model to produce events in the mineral oil, detector structure, and surrounded dirt. The final state particles from the nucleon-level neutrino interactions, in the mineral oil, are propagated through their target nuclei within the neutrino interaction model. These particles are then propagated through the mineral oil producing Cerenkov light, scintillation light, and additional interactions of the produced particles with the mineral oil. The photons interact with the mineral oil through scattering, fluorescence, and extinction before hitting a PMT. The effects of the detector electronics (e.g. discriminator thresholds, time slewings, etc.) are also included in the detector MC.

\subsubsection{Beam}

The MiniBooNE beam MC [33] is constructed within the GEANT4 [41] framework. The simulation is broken into five distinct steps:

- The definition of the BNB geometry through which all particles propagate including the focusing horn, target, and meson decay volume.

- The generation of the primary protons according to the measured beam optic properties. 
- The simulation of the primary p-Be interactions, both elastic and inelastic. Custom tables for protons, neutrons, $\pi^{ \pm}, K^{ \pm}$, and $K^{0}$ were developed based on external data.

- Full tracking of the generated particles within the BNB defined volumes including any tertiary particles that may have been produced, accounting for energy losses, hadron interactions, and decays.

- Identification of the decay processes that result in neutrinos.

The simulated geometry accounts for the last $50 \mathrm{~m}$ of the BNB through the 50 $\mathrm{m}$ meson decay volume. The geometry is defined to match the constructed beamline as accurately as possible. Differences from the actual beamline are noted in Ref. [33].

The horn magnetic field is modeled by a $174 \mathrm{kA}$ current along the inner conductor producing a $1 / R$ field between the conductors with a maximum $1.5 \mathrm{~T}$ field at the narrowest portion. Additionally, a skin-depth effect is simulated to account for the current penetrating a short distance into the conductor.

The primary proton beam is generated $1 \mathrm{~cm}$ upstream of the target. The transverse positions $(x, y)$ are drawn from Gaussian distributions with $0 \mathrm{~mm}$ mean and 1.51 $\mathrm{mm}$ and $0.75 \mathrm{~mm}$ widths respectively. The incident angle is drawn from Gaussian distributions for $\left(\theta_{x}, \theta_{y}\right)$ with $0 \mathrm{mrad}$ means and $0.66 \mathrm{mrad}$ and $0.40 \mathrm{mrad}$ widths respectively. This configuration simulates a divergent beam, even though the true beam is expected to be convergent on the center of the target. However, simulations indicate that this does not affect the expected neutrino flux by more than $1 \%$ [33].

The total cross-sections for proton, neutron, and pion interactions in beryllium and aluminum are set by the Glauber model [42]. Wherever possible, the calculated cross-sections are compared with existing data. The elastic scattering cross-sections are determined by subtracting the inelastic cross-sections from the Glauber total cross- 
section:

$$
\sigma_{T O T}=\sigma_{I N E}+\sigma_{E L A}
$$

where $\sigma_{I N E}$ are the inelastic cross-sections, discussed below, and $\sigma_{E L A}$ are the elastic scattering cross-sections. The total hadron-nucleus cross-section is modeled as a coherent sum of the hadron-nucleon cross-sections calculated by the model. The Glauber model calculates the forward scattering amplitude and extends to the total cross-section by use of the optical theorem. The details of these interactions is discussed in Ref. [33]. The inelastic meson production cross-sections, used to predict the neutrino flux, are explored below.

\subsubsection{Proton Beryllium Interactions}

Primary proton interactions of $8 \mathrm{GeV}$ kinetic energy interact at $\sqrt{s}=4.3 \mathrm{GeV}$ with a stationary beryllium target producing secondary meson particles with masses below the threshold for charm production. However, strange mesons, i.e. kaons, are produced. Custom cross-section parametrizations are used to describe the productions of four of the seven types of secondary particles $\left(\pi^{ \pm}, K^{ \pm}, K^{0}, p, n\right)$ produced off the target. The bulk of the neutrinos at low energies are from $\pi^{+} \rightarrow \mu^{+} \nu_{\mu}$ decays, and the production of $\pi^{+}$is of particular importance and is detailed below. The particles $K^{+}$ and $K^{0}$ contribute to a significant fraction of the intrinsic $\nu_{e}$ flux and are also detailed below. For their contribution to anti-neutrinos, the $\pi^{-}$are important for different beam configurations and is also parametrized below. The production of protons and neutrons is simulated based on the MARS simulation package [43]. The production of $K^{-}$is suppressed relative to $K^{+}$and $K^{0}$, which are already an order of magnitude less than the pion production. The MARS simulation package is used to determine $K^{-}$production as the expected contribution to the neutrino flux is minimal. The production crosssections are completely specified in double-differential form as functions of either $p_{T}$ and $p_{L}$, the transverse and longitudinal momentum, or by $p$ and $\theta$, the magnitude of 
the momentum and the angle relative to the incident beam particle.

\section{Sanford-Wang parametrization}

The $\pi^{ \pm}$and $K^{0}$ production cross-sections are parametrized using a Sanford-Wang (SW) [44] functional form that has been fit to external data near or at MiniBooNE beam energies[33]. The SW parametrization is given by,

$$
\frac{\partial^{2} \sigma}{\partial p \partial \Omega}=c_{1} p^{c_{2}}\left(1-\frac{p}{p_{B}-c_{9}}\right) \exp \left(-c_{3} \frac{p^{c_{4}}}{p_{B}^{c_{5}}}-c_{6} \theta\left(p-c_{7} p_{B} \cos ^{c_{8}} \theta\right)\right)
$$

where $p$ is the momentum of the particle, $\theta$ is the angle, $p_{B}$ is the momentum of the proton beam, and the constants $c_{i}$ are empirically fit to match the various data sets. The $\pi^{ \pm}$data are fit to the HARP [45] and the BNL E910 [46] experiments. The $K^{0}$ production parametrization is fit to the data found in Refs. [46, 47]. Table 2.2 summarizes the parameters found by the fits. The difference in $c_{1}$ between $\pi^{ \pm}$and $K^{0}$ illustrate that $\pi^{ \pm}$dominate the production at this beam energy. Their uncertainties and correlation matrices, used for assessing systematic errors, are given in Ref. [33].

Table 2.2: The Sanford-Wang parameters found by fitting $\pi^{ \pm}$production data $[45,46]$ and $K_{\mathrm{S}}^{0}$ production data $[45,47]$. The covariance matrices are found in Ref. [33].

\begin{tabular}{c|ccccccccc} 
Meson & $c_{1}$ & \multicolumn{1}{c}{$c_{2}$} & $c_{3}$ & $c_{4}$ & $c_{5}$ & $c_{6}$ & $c_{7}$ & $c_{8}$ & \multicolumn{1}{c}{$c_{9}$} \\
\hline \hline$\pi^{+}$ & 220.7 & 1.080 & 1.000 & 1.979 & 1.32 & 5.572 & 0.0868 & 9.686 & 1.00 \\
$\pi^{-}$ & 213.7 & 0.9379 & 5.454 & 1.210 & 1.284 & 4.781 & 0.07338 & 8.329 & 1.00 \\
$K^{0}$ & 15.13 & 1.975 & 4.084 & 0.928 & 0.731 & 4.362 & 0.048 & 13.300 & 1.278
\end{tabular}

\section{Feynman scaling $K^{+}$parametrization}

The data used from $K^{+}$production experiments $[48,49,50,51,52,53,54,55]$ are chosen based on having beam energies close to MiniBooNE's. The beam momenta are over the range $p_{B}=9.5-24 \mathrm{GeV} / c$ compared with $p_{B}=8.89 \mathrm{GeV} / c$ at MiniBooNE. Therefore, the data must be extrapolated down to MiniBooNE's momentum. The parametrization is based on Feynman scaling, which is a hypothesis that the invariant 
cross-section is a function of only two variables, $x_{F}$ and $p_{T}$, where

$$
x_{F} \equiv \frac{p_{\|}}{p_{\|}^{\max }}
$$

is the Feynman scaling variable, $p_{\|}$is the parallel component of the produced particle in the center-of-mass frame, $p_{\|}^{\max }$ is the maximum value for a given reaction, and $p_{T}$ is the produced particles transverse momentum. The double-differential cross-section is given by,

$$
\frac{\partial^{2} \sigma}{\partial p \partial \Omega}=\left(\frac{p_{K^{+}}^{2}}{E_{K^{+}}}\right) c_{1}\left(1-\left|x_{F}\right|\right) \exp \left(-c_{2} p_{T}-c_{3}\left|x_{F}\right|^{c_{4}}-c_{5} p_{T}^{2}-c_{7}\left|p_{T} \times x_{F}\right|^{c_{6}}\right)
$$

where the seven $c_{i}$ are found by fitting to the data in Refs. $[48,49,50,51,52,53,54,55]$. Additionally, the fit is constrained by requiring that $\frac{\partial^{2} \sigma}{\partial p \partial \Omega} \rightarrow 0$ as $x_{F} \rightarrow 1$. The Feynman scaling parameters are summarized in Table 2.3 whose covariance matrix can be found in Ref. [33].

Table 2.3: The Feynman scaling parameters found by fitting $K^{+}$production data [48, $49,50,51,52,53,54,55]$. The covariance matrix is found in Ref. [33].

\begin{tabular}{c|ccccccc} 
Meson & $c_{1}$ & $c_{2}$ & $c_{3}$ & $c_{4}$ & $c_{5}$ & $c_{6}$ & $c_{7}$ \\
\hline \hline$K^{+}$ & 11.70 & 0.88 & 4.77 & 1.51 & 2.21 & 2.17 & 1.51
\end{tabular}

\subsubsection{Neutrino Flux}

The mesons produced in the BNB MC are decayed isotropically in their restframes. The produced neutrinos are boosted into the lab-frame and checked with whether they intersect a circular plane at the detector location but $14 \mathrm{~m}$ in radius. These mesons are stored, and then re-decayed 1000 times to boost the statistics of neutrinos that may possibly interact with the detector without the need to resimulate particles in the BNB. Muons in the secondary beam are boosted by a factor of 20 by making 19 copies that are propagated through the simulation to get a better handle 
on muon decay-in-flight component. The high energy neutrino tail is exponentially enhanced to better populate the high energy region and reduce the statistical fluctuations. The statistical boostings and exponential enhancements are de-weighted in the final flux to get an accurate predication of the flux over the entire energy range. As the kaons contribute mainly to the high energy tail, having a sample in the MC with minimal statistical fluctuations improves the knowledge of kaons in the beam. A method for measuring the kaon content directly in the beam will be discussed in Chapter 3 .

As there are no in-situ methods to measure the incident neutrino flux at MiniBooNE, the experiment relies completely on the flux prediction. Any direct measurement of the neutrinos are convoluted with neutrino interactions cross-sections; crosssections that the experiment is trying to measure. The only way to measure to the flux directly would be to measure the production of pions, kaons, and muons in the secondary beam. For the bulk of the flux, neutrinos from pion decays, this was effectively performed by the HARP experiment [45]. They took a thin replica of the MiniBooNE beryllium target, and then impacted the replica with $8 \mathrm{GeV}$ protons, measuring the produced charged pions. However, the re-interactions of both protons and pions in a long target were not measured. For this, the Glauber [42] model is trusted. Additionally, the focusing of charged particles caused by the horn is estimated by Ampere's law, and verified by several field measurements taken within the horn cavity. The focusing was also checked by running the experiment with different amounts of horn current and comparing the changing rates in the detector. All of these effects contribute to possible variations in the absolute neutrino flux. The systematic uncertainties associated with flux are addressed in §5.4.2. An accurate muon neutrino flux is important for extracting the muon neutrino interaction cross-sections, as this dissertation attempts. Fig. 2.14 shows the predicted neutrino flux for each neutrino species at the MiniBooNE detector. The $\nu_{\mu}$ are by far the most abundant. 


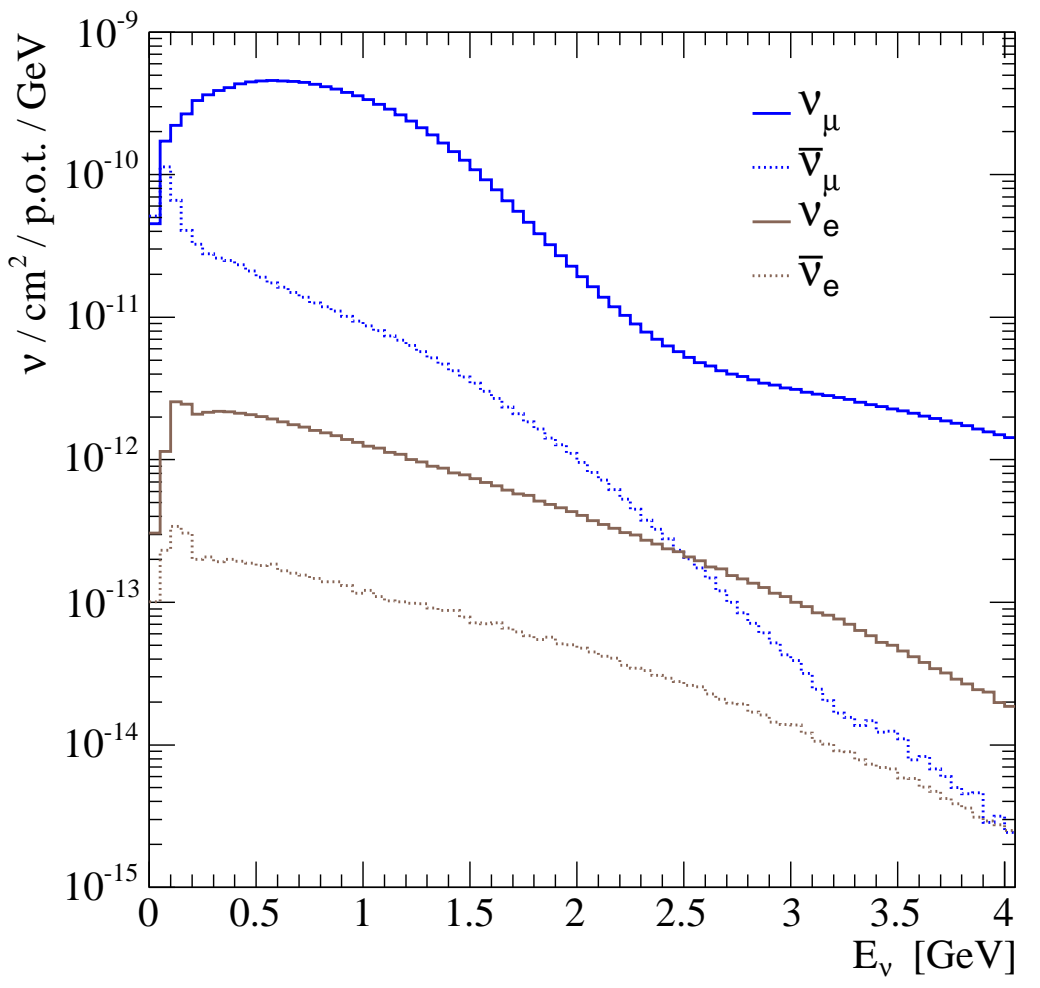

Figure 2.14: The predicted neutrino flux for each species as a function of neutrino energy.

\subsubsection{Detector}

The MiniBooNE detector MC is simulated within the GEANT3 [56] framework. The detector MC receives as input the neutrino flux for each species. The MC interacts those neutrinos, based on a comprehensive cross-section model, with the mineral oil. Included within the cross-section model are interactions of both the final state particles and the nuclear resonances with the spectator nucleons. Once the particles exit the target nucleus, they are subject to interactions with the mineral oil simulated with GCALOR ??. These interactions either produce photons that propagate through the mineral oil until they encounter the detector's PMTs, or affect the particle in some drastic manner (e.g. hard scatters, absorption, etc.). The photons are also subject to 
interactions with the mineral oil. As the detection of photons that make it to the tank walls provide the only source of information about the interaction, photon interactions in the mineral oil are extremely important. The photon interactions with the PMTs are also simulated, along with the effects on the PMT read-out with the electronics.

A random "strobe" trigger records the detector activity during periods of no beam. Strobe events are overlaid with the MC to mock up the effects of ambient radioactivity, coincident cosmic ray events, and other non-simulated sources of light.

\subsubsection{Cross-sections}

The NUANCE [57] neutrino event generator is a comprehensive simulation of 99 of the most relevant interactions for neutrinos and anti-neutrinos from over an energy range from $100 \mathrm{MeV}$ to $1 \mathrm{TeV}$.

\section{Quasi-elastic scattering}

The charged-current quasi-elastic (CCQE) model used by NUANCE is that of the Llewellyn Smith model [58]. The nucleon form factors are parametrized in a dipole form as functions of $Q^{2}$. The vector, axial-vector, and pseudo-scalar form factors are explicit and depend on two mass constants: the vector mass and the axial mass. The model is given by [59],

$$
\frac{d \sigma}{d Q^{2}}=\frac{G_{\mathrm{F}}^{2} M^{2}}{8 \pi E_{\nu}^{2}}\left[A\left(Q^{2}\right)-\frac{(s-u)}{M} B\left(Q^{2}\right)+\frac{(s-u)^{2}}{M^{2}} C\left(Q^{2}\right)\right]
$$

where $G_{F}$ is Fermi's constant, $M$ is the target nucleon's mass, and $E_{\nu}$ is the neutrino energy in the lab frame. The Mandelstam variables can be expressed as,

$$
s-u=4 M E_{\nu}-Q^{2}-m_{l}^{2}+\left(M^{2}-M^{\prime 2}\right)
$$

where $M^{\prime}$ is the mass of the scattered nucleon, and $m_{l}$ is the lepton mass. The term in 
parenthesis is neglected in the model. The functions, in terms of the form factors, are,

$$
\begin{aligned}
A\left(Q^{2}\right)= & {\left[\frac{m_{l}^{2}}{M^{2}}+4 \tau\right]\left[(1+\tau) F_{A}^{2}-(1-\tau) F_{1}^{2}+\tau(1-\tau) F_{2}^{2}+4 \tau F_{1} F_{2}\right.} \\
& \left.\left.-\frac{m_{l}^{2}}{4 M^{2}}\left(\left(F_{1}+F_{2}\right)^{2}+\left(F_{A}+2 F_{P}\right)^{2}-4(1+\tau) F_{P}^{2}\right)\right)\right] \\
B\left(Q^{2}\right)= & 4 \tau F_{A}\left(F_{1}+F_{2}\right) \\
C\left(Q^{2}\right)= & \frac{1}{4}\left(F_{A}^{2}+F_{1}^{2}+\tau F_{2}^{2}\right)
\end{aligned}
$$

where $\tau \equiv Q^{2} / 4 M^{2}, F_{A}$ is the axial-vector form factor, $F_{1,2}$ are the vector form factors, and $F_{P}$ is the pseudo-scalar form factor. The form factors are given by,

$$
\begin{aligned}
& F_{1}\left(Q^{2}\right)=\frac{1+\tau\left(1+\mu_{p}-\mu_{n}\right)}{(1+\tau)\left(1+\frac{Q^{2}}{m_{V}^{2}}\right)^{2}} \\
& F_{2}\left(Q^{2}\right)=\frac{\left(\mu_{p}-\mu_{n}\right)}{(1+\tau)\left(1+\frac{Q^{2}}{m_{V}^{2}}\right)^{2}} \\
& F_{A}\left(Q^{2}\right)=\frac{g_{A}}{\left(1+\frac{Q^{2}}{m_{A}^{2}}\right)^{2}} \\
& F_{P}\left(Q^{2}\right)=\frac{2 M^{2}}{m_{\pi}^{2}+Q^{2}} F_{A}\left(Q^{2}\right)
\end{aligned}
$$

where $g_{A}=-1.25, m_{V}=0.84 \mathrm{GeV} / \mathrm{c}^{2}$ is the vector mass, $m_{A}=1.03 \pm 0.02 \mathrm{GeV} / \mathrm{c}^{2}$ is the axial mass [60], $m_{\pi}$ is the pion mass, and $\mu_{p, n}$ are the proton and neutron anomalous magnetic moments.

The CCQE model has been significantly tuned to match the measured $\nu_{\mu}$-CCQE cross-sections. The axial mass is tuned to $M_{A}^{\mathrm{eff}}=1.23 \pm 0.20 \mathrm{GeV}$ [61]. Additional tunings to the CCQE model are relevant to the nuclear interaction model and will be addressed later in this section.

\section{Single pion production}

The Rein-Seghal (RS) model used in NUANCE provides a comprehensive description of pion production processes for both neutrino and anti-neutrino scattering off nuclei [62]. The model incorporates all non-strange baryon resonances with masses below $2 \mathrm{GeV} / c^{2}$, including all interference terms, to describe 14 neutrino and anti-neutrino 
processes. Additionally, a single non-interfering, non-resonant background of isospin $1 / 2$ has been included to improve agreement with experiments. Like the CCQE model, the RS model parametrizes the form factors as dipoles separating out the vector, axialvector, and pseudo-scalar components. The quarks are modeled as relativistic harmonic oscillators with the formalism developed by Feynman-Kislinger-Ravndal (FKR) [63].

The $\mathrm{CC}$ and $\mathrm{NC}$ resonances are defined by the interactions $\nu N \rightarrow l N^{*}$ and $\nu N \rightarrow \nu N^{*}$ where $N$ is the target nucleon and $N^{*}$ is one of 18 considered nucleon resonances. However, at MiniBooNE energies $\Delta(1232)$ is the dominant resonance. The transition amplitudes for these processes are given by the Feynman rules as,

$$
\begin{aligned}
T\left(\nu N \rightarrow l N^{*}\right) & =\frac{g^{2} \cos \theta_{c}}{8}\left[\bar{u}_{l} \gamma^{\alpha}\left(1-\gamma^{5}\right) u_{\nu}\right]\left\{\frac{-g_{\alpha \beta}+\frac{q_{\alpha} q_{\beta}}{M_{W}^{2}}}{q^{2}-M_{W}^{2}}\right\}\left\langle N^{*}\left|J^{\beta}\right| N\right\rangle \\
T\left(\nu N \rightarrow \nu N^{*}\right) & =\frac{g^{2}}{8 \cos ^{2} \theta_{W}}\left[\bar{u}_{\nu} \gamma^{\alpha}\left(1-\gamma^{5}\right) u_{\nu}\right]\left\{\frac{-g_{\alpha \beta}+\frac{q_{\alpha} q_{\beta}}{M_{Z}^{2}}}{q^{2}-M_{Z}^{2}}\right\}\left\langle N^{*}\left|J^{\beta}\right| N\right\rangle
\end{aligned}
$$

where the hadronic current $\left\langle N^{*}\left|J^{\beta}\right| N\right\rangle$ will be parametrized by the RS model. In the regime of low $Q^{2}<2 \mathrm{GeV}$, the amplitudes simplify to,

$$
\begin{aligned}
T\left(\nu N \rightarrow l N^{*}\right) & =\frac{G_{F} \cos \theta_{c}}{\sqrt{2}}\left[\bar{u}_{l} \gamma^{\alpha}\left(1-\gamma^{5}\right) u_{\nu}\right]\left\langle N^{*}\left|J_{\alpha}\right| N\right\rangle \\
T\left(\nu N \rightarrow \nu N^{*}\right) & =\frac{G_{F}}{\sqrt{2}}\left[\bar{u}_{\nu} \gamma^{\alpha}\left(1-\gamma^{5}\right) u_{\nu}\right]\left\langle N^{*}\left|J_{\alpha}\right| N\right\rangle
\end{aligned}
$$

where $M_{Z}^{2} \cos ^{2} \theta_{W} \simeq M_{W}^{2}$ and $G_{F} / \sqrt{2}=g^{2} / 8 M_{W}^{2}$. Making the additional assumptions that the lepton masses are small, and that $\cos \theta_{c} \sim 1$ the expressions can be combined to describe the production of nucleon resonances for both $\mathrm{CC}$ and $\mathrm{NC}$ modes as,

$$
T\left(\nu N \rightarrow x_{l} N^{*}\right)=\frac{G_{F}}{\sqrt{2}}\left[\bar{u}_{x_{l}} \gamma^{\alpha}\left(1-\gamma^{5}\right) u_{\nu}\right]\left\langle N^{*}\left|J_{\alpha}\right| N\right\rangle
$$

where $x_{l}=\nu, l$. The cross-section for a single resonance is then,

$$
\frac{\partial^{2} \sigma}{\partial Q^{2} \partial E_{q}}=\frac{1}{128 \pi^{2} M_{N} E_{\nu}^{2}} \sum_{\text {spins }}\left|T\left(\nu N \rightarrow x_{l} N^{*}\right)\right|^{2} \frac{\Gamma}{\left(W-M_{N^{*}}\right)^{2}-\Gamma^{2} / 4}
$$

where $W$ is the observed resonance mass, $\Gamma$ is the decay width, and $E_{q}$ is the energy of the virtual $W^{ \pm}$or $Z^{0}$. However, the goal of the RS model is to include the interferences 
of the resonances. The fact that the widths $\Gamma$ are significant compared to the separation between resonance masses means that nearby nucleon resonances will overlap. The resonances are interfered where appropriate based on the selection rules allowable to the interactions and the 75 transition amplitudes are calculated [62].

Within the context of the FKR relativistic harmonic oscillator model, the transition form factors for single pion production by neutrino scattering off nuclei are,

$$
F_{A, V}\left(Q^{2}\right)=\left(1+\frac{Q^{2}}{4 M_{N}^{2}}\right)^{1 / 2-n}\left(1+\frac{Q^{2}}{m_{A, V}^{2}}\right)^{2}
$$

where the vector mass is $m_{V}=0.84 \mathrm{GeV} / c^{2}$, the axial mass has been tuned to $m_{A}=$ $1.10 \pm 0.27 \mathrm{GeV} / c^{2}[61]$, and $n$ is the number of oscillators in the final resonance. The first term in parenthesis was added ad-hoc to help agreement to data [62].

\section{Nuclear interactions}

The nucleus is simulated as a relativistic Fermi gas (RFG) according to the SmithMoniz model [64]. The RFG models the nucleons as having a flat momentum spectrum in the nucleus up to a cut-off called the Fermi momentum, $p_{F}$. The nucleons are also bound with binding energy $E_{B}$. Additionally, the effect of nuclear shadowing is incorporated through a "Pauli blocking" parameter $\kappa$. This parameter causes a suppression of the cross-section at low $Q^{2}$ as the target nucleon is forbidden from entering the same state as one of the spectator nucleons. These parameters are: $p_{F}=220 \pm 30 \mathrm{MeV} / c$, $E_{B}=34 \pm 9 \mathrm{MeV}$, and $\kappa=1.019 \pm 0.011$ [61]. A $\kappa=1$ would have implied no Pauli blocking.

Resonant pion production is also subject to the RFG model. The resonances can travel $\sim 1 \mathrm{fm}$; a significant difference across the nucleus. The $N^{*} N \rightarrow N N$ interactions are tuned using K2K data with $100 \%$ assumed uncertainty [61]. Additionally, final state pion interactions are included as $\pi^{+}$charge exchange $\left(\pi^{+} N \rightarrow \pi^{0} N^{\prime}\right)$ and absorption $\left(\pi^{+} N \rightarrow \lambda^{+} N^{\prime}\right)$. The final state pion nuclear effects differ from the scattering processes that charge exchange or absorb pions in the mineral oil. Those processes are discussed 
in $\S 5.15$.

\subsubsection{Optical Model}

Particles traversing the mineral oil produce light through several processes. The emitted photons are also subject to interactions with the mineral oil. The simulation of these processes are referred to collectively as the "optical model," and its various parameters have been measured either externally, or through in-situ measurements.

Careful measurements of the index of refraction in the oil [65] suggest a dependence on wave length and temperature of,

$$
n(\lambda, T)=\left[n_{D}+C_{0}\left(\frac{1}{\lambda^{2}}-\frac{1}{\lambda_{D}^{2}}\right)\right] \cdot\left[1-C_{1}\left(T-T_{0}\right)\right]
$$

where $C_{0}, C_{1}, n_{D}, \lambda_{D}$ and $T_{0}$ are summarized in Table 2.4. Typical values of $\lambda$ and $T$ give $n=1.54$.

Table 2.4: Summary of the parameters describing the index of refraction in Marcol 7 found in Ref. [65].

\begin{tabular}{c|cl} 
Parameter & value & note \\
\hline \hline$\lambda_{D}$ & $589.3 \mathrm{~nm}$ & wavelength of Na doublet (test laser) \\
$T_{0}$ & $293.15 \mathrm{~K}$ & \\
$n_{D}$ & $1.4684 \pm 0.0002$ & refractive index at $\lambda_{D}$ and $T_{0}$ \\
$C_{0}$ & $4240 \pm 157 \mathrm{~nm}^{2}$ & \\
$C_{1}$ & $(3.66 \pm 0.04) \times 10^{-4} \mathrm{~K}^{-1}$ &
\end{tabular}

\section{Direct sources of light}

Črenkov radiation $[66,67]$ is a consequence of photon interactions within a medium. A photon in a medium is influenced by the local electromagnetic fields, which add constructively to the photon causing a suppression of the photon's wavelength while leaving the frequency unaffected. This effect allows for charged particles, under certain conditions, to spontaneously emit photons, which is referred to as Čerenkov radiation. Fig. 2.15 shows the process of a charged particle emitting a photon in a medium.

\footnotetext{
${ }^{1}$ This is a distinctly different process from bremsstrahlung, which occurs because of interactions of
} 
Conserving four-momentum between the initial and final state gives,

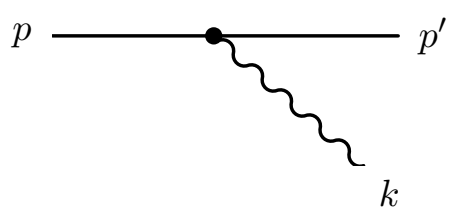

Figure 2.15: A charged particle passing through, or near a medium can radiate in the medium. The initial momentum of the charged particle is given by $p$, the final momentum by $p^{\prime}$. The radiated photon's momentum is given by $k$.

$$
p=p^{\prime}+k
$$

where $p$ is the particle's initial momentum, $p^{\prime}$ is the particle's final momentum, and $k$ is the momentum of the photon. Rearranging and squaring the expression becomes,

$$
p \cdot k=\frac{k^{2}}{2}
$$

As the photon's momentum is greater than its energy it is no longer a null vector, the photon has become spacelike. While this may seem to be a contradiction, Lorentz boosts are still unable to cause the photon's momentum to vanish. Solving the expression for the angle between the photon and the direction of the particle before the photon was emitted gives,

$$
\cos \theta=\frac{2 E \epsilon-k^{2}}{2|\mathbf{p}||\mathbf{k}|}
$$

where $\epsilon$ is the energy of the photon, and $|\mathbf{p}| / E=\beta$ is the velocity of the particle. For a photon the energy is given by $\epsilon=h \nu$ where $h$ is Planck's constant and $\nu$ is the photon frequency. The photon's momentum is $|\mathbf{k}|=h / \lambda$ where $\lambda$ is the photon's wavelength. In vacuum it is clear that $\epsilon /|\mathbf{k}|$ is the photon's velocity. In a medium the photon's frequency does not change but the wavelength shortens. This implies that the speed of light in a the charged particle with the medium and can be a significant source of energy loss. Čerenkov radiation is the spontaneous emission of photons and does not cause much energy loss. 
medium is less than the speed of light in vacuum, and the ratio $n=c_{\text {vacuum }} / c_{\text {medium }}$ is the index of refraction ( $n \geq 1$ for "normal" materials). Therefore, in a material, the ratio $|\mathbf{k}| / \epsilon$ is recognized as the index of refraction $n$. The angle of Cerenkov emission derived in Eqn. 2.25 becomes,

$$
\cos \theta=\frac{1}{n \beta}\left[1+\frac{\epsilon}{2 E}\left(n^{2}-1\right)\right] \simeq \frac{1}{n \beta}
$$

The second term is due to quantum corrections [68] of the classical result. As the energy of the Čerenkov photons are typically optical $(\mathrm{O}(\mathrm{eV}))$ and the charged particles of interest have energies greater than an $\mathrm{MeV}$, the term proportional to $\epsilon / E$ can effectively be ignored. From this equation it is clear that Čerenkov radiation occurs if the particle's velocity is greater than the local speed of light ${ }^{2}$ as there is no real solution otherwise. The rate of production is given by [20],

$$
\frac{d^{2} N}{d x d \epsilon}=\alpha Z^{2}\left(1-\frac{1}{\beta^{2} n^{2}(\epsilon)}\right)
$$

where $\alpha \simeq 1 / 137$ is the fine structure constant, and $x$ is the path of the charged particle. The amount of Čerenkov radiation is adjusted by an overall scale factor in the MC to match the rate in calibration samples. The scale factor, $f_{C h e}=1.106$, suggests a $10 \%$ difference.

The other source of direct light is due to scintillation of the mineral oil. Scintillation occurs when the liberated electrons from the ionization of the medium recombine into an unoccupied excited state. The subsequent photon released during the recombination is then transparent to the medium. The emitted photons are isotropic. The number of liberated photons is given by Birk's law as a function of deposited energy in the medium by,

$$
\frac{d N}{d E}=\frac{A}{1+B_{1} \frac{d E}{d x}+B_{2}\left(\frac{d E}{d x}\right)^{2}}
$$

\footnotetext{
${ }^{2}$ Actually, Eqn. 2.26 implies that the particle needs a velocity slightly larger than the local speed of light.
} 
where $A=31.64 \mathrm{MeV}^{-1}, B_{1}=\left(0.014 / \rho_{\text {oil }}\right) \mathrm{cm} / \mathrm{MeV}$, and $B_{2}=\left(0.0 / \rho_{\text {oil }}^{2}\right) \mathrm{cm}^{2} / \mathrm{MeV}^{2}$. The parameter $B_{2}$ is included only for the assessment of systematic errors. There is a delay of emission on the order of tens of nanoseconds.

\section{Optical properties of Marcol 7}

As the direct light propagates through the mineral oil it is subject to several different effects: scattering, fluorescence, and absorption. The two types of scattering considered are elastic Rayleigh scattering and inelastic Raman scattering. Rayleigh scattering is caused by the absorption of a photon by a ground state electron that immediately decays back to the ground state emitting a photon of the same energy, hence elastic. This is not an isotropic scatter and the intensity of the Rayleigh scattering distribution is proportional to $1+\cos ^{2} \theta$ and $1 / \lambda^{4}$.

Raman scattering and fluorescence occur through the same process as scintillation, though their causes are slightly different. As scintillation is a direct effect caused by the ionization of the incident particle, Raman scattering and fluorescence occur by the scattering of photons. All of these processes require an excited state of a molecule in the medium somewhat near the ground state that has an allowable transition from the fully ionized state to that excited state. However, fluorescence describes the resonant portion of the interaction, exciting the molecule with the electron staying bound, till it eventually decays down into a low lying excited state. Raman scattering ionizes the atom and the photon is emitted at recombination, again to a low lying excited state. There are 4 known fluors in mineral oil. Fig. 2.16 summarizes the knowledge of light propagation in Marcol 7 based on external measurements and calibration data. Fluor 1 dominates the fluorescence spectrum.

\section{The Optical Model}

The combination of all of the above effects, including PMT efficiencies, are incorporated into a 35-parameter custom optical model. However, only 21 of the parameters are incorporated in the central value optical model. Three of the fluorescence modes are 


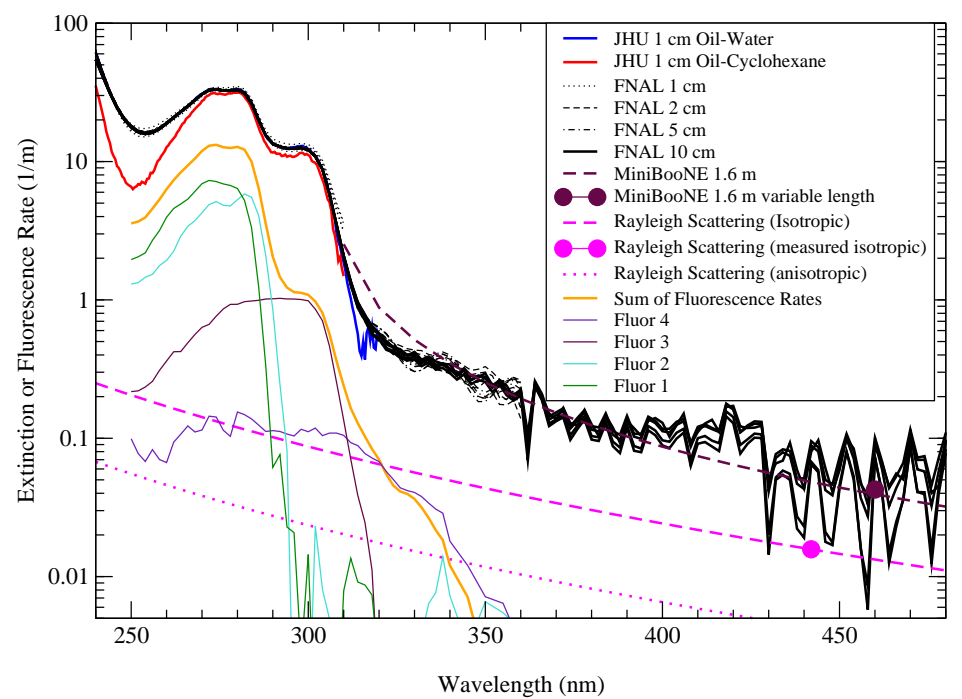

Figure 2.16: Optical photon extinction rates in Marcol 7. Extinction is referred to any process that alters the state of a photon. Wavelengths below $250 \mathrm{~nm}$ in the UV spectrum are not visible to the PMTs. Rayleigh scattering was measured at specific wavelengths and extrapolated by the known $\lambda^{4}$ dependence.

excluded along with the UV fluorescence of fluor 1, as well as coefficient $B_{2}$ of Birk's law. The remaining parameters are included in the assessment of systematic errors and will be discussed in $§ 5.4 .3 .3$. The optical model is included within the GEANT3 simulation.

\subsection{Discussion}

The original proposed BooNE experiment consisted of both near and far detectors. For funding reasons, the experiment was scaled down to the single detector described in this chapter. The advantages of two detectors were mainly for the measurement of neutrino oscillations. The near detector would have been able to measure the rate of neutrino interactions prior to oscillations; the far detector would have measured the rate post oscillations. The advantage is that the ratio of the two measurements cancel out both the flux and the cross-sections, assuming that both detectors had similar 
systematics, and left only the effect due to oscillations. Unfortunately this was not the case. The only way MiniBooNE could do either a $\nu_{e}$ appearance experiment, or a $\nu_{\mu}$ disappearance experiment were to have excellent particle-ID and an understood flux. Years of work went into understanding the flux and the optical model for these very reasons.

While MiniBooNE was not able to constrain oscillation parameters as well as it would have been able to with two detectors, the single-detector experiment turned into an ideal neutrino cross-section experiment. MiniBooNE has the largest sample of neutrinos ever recorded in this energy range. The same would have been true of BooNE, however, the heroic efforts involved to understand the flux and optical model probably would not have been undertaken. MiniBooNE has measured cross-sections for five interaction modes, covering $89 \%$ of the total rate and $96 \%$ of the chargedcurrent modes. The dominant interaction, CCQE, comprises $44 \%$ of the total rate and measurements of the cross-section are discussed in Refs. $[69,61,70]$. The next largest mode, $\mathrm{CC} \pi^{+}$, comprises $19 \%$ of the total rate and the cross-section measurements are discussed in Refs. [71, 72]. The neutral-current elastics (NCEL) comprise $17 \%$ of the rate; cross-section measurements are discussed in Refs. [73, 74]. $\mathrm{NC} \pi^{0}$ make up $5 \%$ of the interactions; measurements of the cross-sections are in Ref. [75]. Finally, $\mathrm{CC} \pi^{0}$ interactions make up $4 \%$ of the rate; the measurements are the subject of this dissertation.

The MiniBooNE cross-section measurements provide the most complete understanding of neutrino interactions at $E_{\nu} \in(300-2000 \mathrm{MeV})$ to date. These measurements will aid in the prediction of event rates for the next generation of neutrino oscillation experiments. Additionally, having many measurements from the same experiment aids in the understanding of nuclear effects, or final state interactions. 


\section{Chapter 3}

\section{The Little Muon Counter}

The "Little Muon Counter" (LMC) as it was lovingly called, was an attempt to constrain the normalization of the kaon content of the secondary meson beam. Kaons, through the $K_{e 3}^{+}$decay mode, are a source of intrinsic $\nu_{e}$. Kaons provide $44 \%$ of the intrinsic $\nu_{e}$ flux [33]. The absolute production of kaons off of the beryllium target is uncertain, and the $K^{+}$production component contributes the largest single uncertainty to the $\nu_{e}$ flux at $11.5 \%$ [33]. Fig. 3.1 shows the predicted neutrino fluxes divided by $\nu_{\mu, e}$
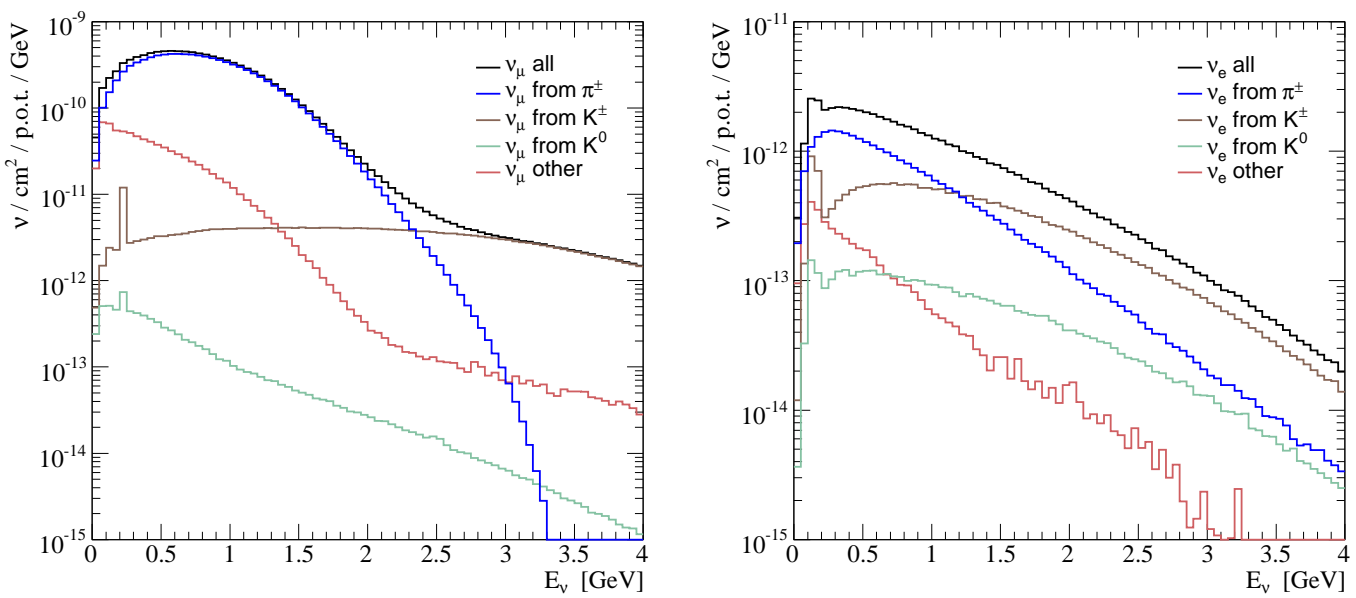

Figure 3.1: Left: The predicted $\nu_{\mu}$ flux separated by neutrino parent. Pion parents completely dominate the flux below $2.3 \mathrm{GeV}$, with kaon parents dominating at high energies. Right: The intrinsic $\nu_{e}$ flux separated by neutrino parent. The kaon parent contribution is significant across all energies and dominates above $1.2 \mathrm{GeV}$. 
and by parent species ${ }^{1}$. The goal is to tie the level of intrinsic $\nu_{e}$ from kaon decays to a measurement of the $\mu^{+}$from kaon decay spectrum.

This chapter details the design, construction, MC, reconstruction, and analysis of the LMC in an attempt to understand the kaon content of the MiniBooNE beam. This study is separate from the primary result of this dissertation, the $\mathrm{CC} \pi^{0}$ cross-section measurements.

\section{$3.1 \quad$ Concept}

As pions and kaons have significantly different masses, $139.57 \mathrm{MeV} / c^{2}$ and 493.68 $\mathrm{MeV} / \mathrm{c}^{2}$ respectively [20], their decay products have different momentum in their respective rest-frames. For a meson beam, this translates into different allowable ranges for their decay products' transverse momentum. The MiniBooNE secondary meson beam consists mostly of $\pi^{+}$with some $K^{+}$and $K_{\mathrm{L}}^{0}$ contributions. The maximum allowable transverse momenta for the relevant pion and kaon decays show a difference of a factor of 7 in maximum transverse momentum for a muon. A detector positioned at an angle off-axis from the secondary beam, might be able to distinguish the difference between the transverse momentum distributions.

\section{Two-body decays}

The maximum transverse momentum, $p_{\mathrm{T}}$, that a particle can have in the lab frame is the maximum allowable momentum in the rest frame of the decaying particle. For two-body decays where

$$
p-p_{1}=p_{2},
$$

the momenta of $p_{1}$ and $p_{2}$ are equal and opposite in the rest frame of $p$. Solving for $\left|\mathbf{p}_{1}\right|$ the momentum is,

$$
p_{\mathrm{T}}^{\max } \equiv\left|\mathbf{p}_{1}\right|=\frac{\sqrt{\Delta\left(m^{2}, m_{1}^{2}, m_{2}^{2}\right)}}{2 m}
$$

\footnotetext{
${ }^{1} \pi^{+} \rightarrow \mu^{+} \rightarrow \ldots \rightarrow \nu$ are included in the $\pi^{+}$contribution.
} 
Table 3.1: Dominant decay modes with $\mu^{+}$in the final state for $\pi^{+}$and $K^{+}$. Branching fractions from Ref. [20].

\begin{tabular}{ccc} 
Decay & $p_{\mathrm{T}}^{\max }[\mathrm{MeV} / c]$ & Branching Fraction \\
\hline \hline$\pi^{+} \rightarrow \mu^{+} \nu_{\mu}$ & 29.8 & $99.98770 \pm 0.00004 \%$ \\
$K^{+} \rightarrow \mu^{+} \nu_{\mu}$ & 235.5 & $63.44 \pm 0.14 \%$ \\
$K^{+} \rightarrow \pi^{0} \mu^{+} \nu_{\mu}$ & 213.7 & $3.32 \pm 0.06 \%$
\end{tabular}

where the "triangle" function is defined by,

$$
\Delta(a, b, c) \equiv a^{2}+b^{2}+c^{2}-2 a b-2 a c-2 b c
$$

clearly, Eqn. 3.2 is symmetric with the interchange of $1 \leftrightarrow 2$ as was expected for equal and opposite momentum.

\section{Three-body decays}

For three-body decays conservation of momentum yields,

$$
p-p_{1}=p_{2}+p_{3}
$$

where the final state momenta are not equal and opposite. The goal is to find the maximum $p_{1}$ in the rest-frame of $p$. By defining $m_{23}^{2} \equiv\left(p_{2}+p_{3}\right)^{2}$ the derivation is identical to Eqn. 3.2,

$$
\left|\mathbf{p}^{\mathrm{T}}\right| \equiv \frac{\sqrt{\Delta\left(m^{2}, m_{1}^{2}, m_{23}^{2}\right)}}{2 m}
$$

where $m_{23}^{2}$ has been substituted for $m_{2}^{2}$. This equation is maximum when $m_{23}^{2}$ is smallest. The minimum value is when $m_{23}^{2}=\left(m_{2}+m_{3}\right)^{2}$; both particles have the same velocity.

Table 3.1 summarizes the maximum muon $\left|\mathbf{p}^{\mathrm{T}}\right|$ and branching fractions for several pion and kaon decay modes. As the distribution of transverse muon momentum is higher for kaons than it is for pions, the kinematics of the meson beam are important for understanding the distribution of transverse muon momentum from the decays of either species.

The MiniBooNE beam MC implied that an off-axis angle of $7^{\circ}$ is ideal to gauge the difference between the pion and kaon contributions in the secondary beam. Fig. 3.2 


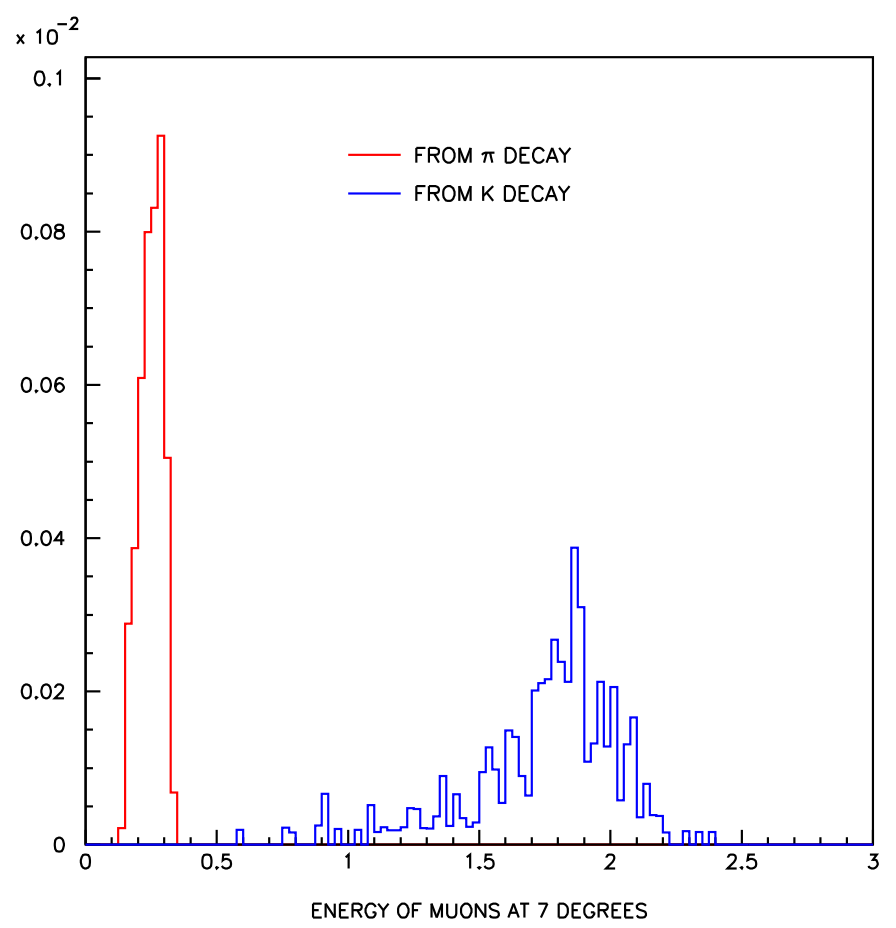

Figure 3.2: The predicted energy spectrum of muons from pion parents (red) and kaon parents (blue) at $7^{\circ}$ off-axis the MiniBooNE beamline.

shows the true energy spectrum for pion and kaon parents at a $7^{\circ}$ off-axis angle. The energy of muons from pion parents is tightly peaked at $\sim 250 \mathrm{MeV}$ where the muons from kaons have a broad spectrum with a peak around $1.8 \mathrm{GeV}$. The pion parents that end up in the sample must have gone through some scattering in the air, as most unscattered pions are unable to send a muon at an angle of $7^{\circ}$.

\subsection{Fiber Hodoscope}

A scintillating fiber spectrometer was positioned at $7^{\circ}$ off-axis to measure the momentum of muons from decays in the secondary beam. The system consisted of an evacuated drift pipe, a steel/tungsten collimator, a scintillating veto, a fiber spectrometer, and a muon range stack. The system was designed to isolate a sample of muons 
from hadronic backgrounds and accurately measure their momentum. Fig. 3.3 shows a

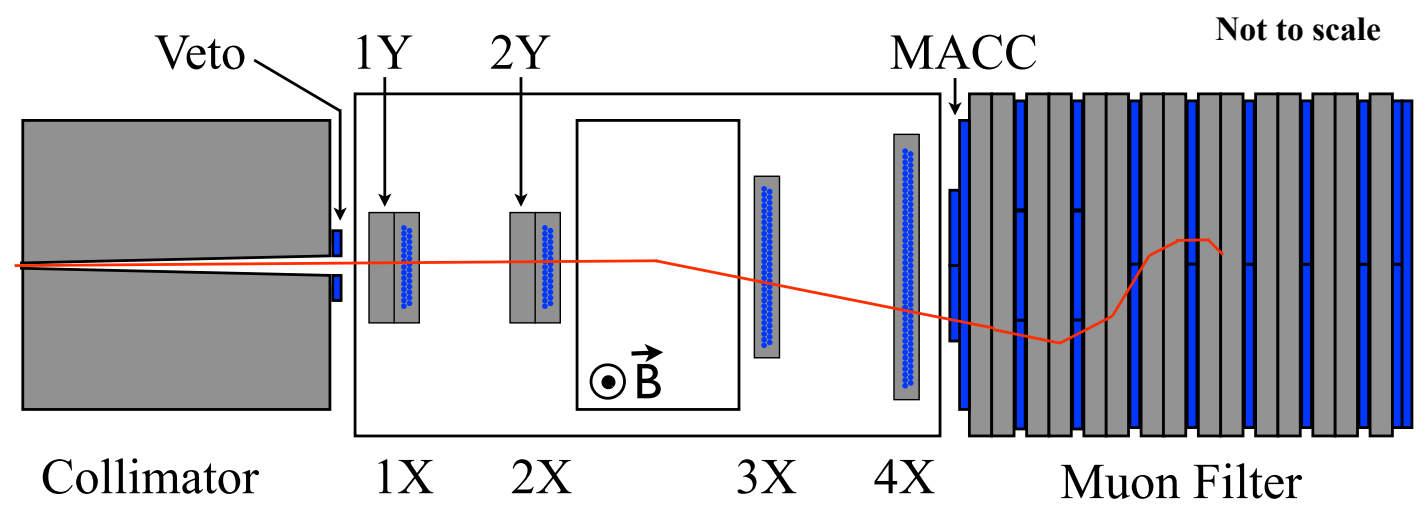

Figure 3.3: Top view of the LMC. Muons come from the drift pipe (not shown) and enter a steel and tungsten collimator. On the face of the collimator is a scintillating veto. A fiber hodoscope measures the momentum of charge particles as they transit a dipole magnetic field with 6 planes of scintillating fibers. A secondary veto selects clean track through the hodoscope (MACC). Muons are selected based on their penetration depth into the range stack. A cartoon of a muon is shown in red.

diagram of the LMC apparatus. The design, calibration, and simulation of the LMC are detailed.

\subsubsection{Apparatus}

An evacuated drift pipe taken at $7^{\circ}$ off-axis the secondary beamline points back into the 25 meter absorber enclosure. At the far end of the drift pipe is located a steel collimator with a tungsten core. Attached to the downstream face of the collimator is a scintillator paddle veto to tag events that passed through the material of the collimator. Immediately after the collimator is the upstream portion of the fiber spectrometer; a series of 4 planes of scintillating fibers at two positions. At each position there are two planes, one to tag the horizontal position, the other the vertical position. After these 4 planes of fiber-optics is a custom $0.223 \mathrm{~T}$ dipole magnet (vertical field). Downstream of the magnet are two more fiber planes, at two positions, to measure the horizontal bend 
of the particle caused by the dipole field. Behind the fiber tracker is a muon range stack of interleaved planes of tungsten and scintillator. Fig. 3.4 is a photograph of the LMC.

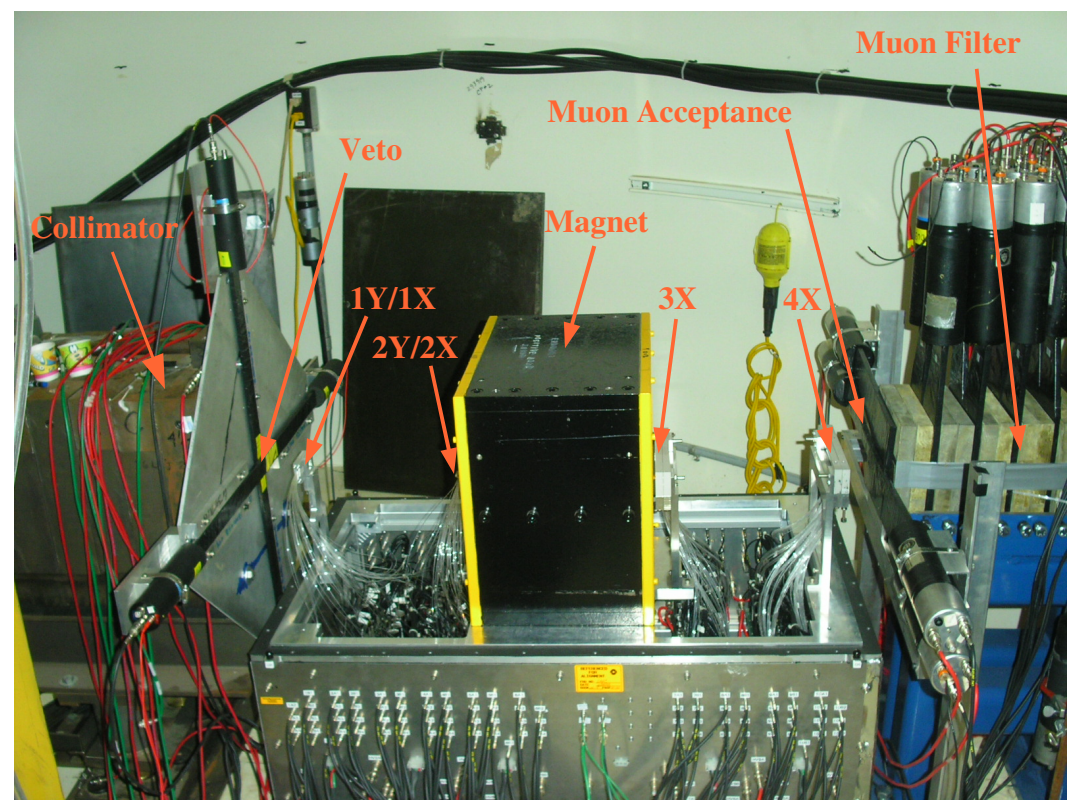

Figure 3.4: A photograph of the LMC. Muons enter from the left, through the collimator. The fiber tracker is shown with its light-tight lid removed to display the fiber planes and dipole magnet.

The coordinate system is chosen such that the $z$-axis is along the collimator direction, the $y$-axis is vertical, and the $x$-axis is beam left (as defined by the "righthand" rule). The center of the dipole magnet is defined to be at $z=0$. Positions upstream of the magnet are at negative $z$; downstream at positive $z$.

\subsubsection{Drift Pipe}

The drift pipe intersects the meson decay volume at an angle of $7^{\circ}$, at $41 \mathrm{~m}$ from the target, looking into the $25 \mathrm{~m}$ absorber enclosure. The angle is $1.8^{\circ}$ upward, and $6.8^{\circ}$ off the beam center-line (horizontal). The pipe is a 60 foot long, 8 inch diameter gas pipe. The ends of the pipe are sealed by thin vacuum windows with the air pressure in 
Table 3.2: The alloy composition of Elgiloy. The average atomic number is, $\langle\mathrm{Z}\rangle=27.41$, and weight, $\langle\mathrm{A}\rangle=59.54$. The density, $\rho=6.345 \pm 0.439 \mathrm{~g} / \mathrm{cm}^{3}$, was measured using a buoyancy tank.

\begin{tabular}{crrl} 
Element & Fraction & $\mathrm{Z}$ & \multicolumn{1}{c}{$\langle\mathrm{A}\rangle$} \\
\hline \hline $\mathrm{Co}$ & $40 \%$ & 27 & 58.933 \\
$\mathrm{Cr}$ & $20 \%$ & 24 & 51.996 \\
$\mathrm{Ni}$ & $15.5 \%$ & 28 & 58.69 \\
$\mathrm{Mo}$ & $7 \%$ & 42 & 95.95 \\
$\mathrm{Mn}$ & $2 \%$ & 25 & 54.94 \\
$\mathrm{Fe}$ & balance & 26 & 55.85
\end{tabular}

the pipe kept below 0.1 torr. The window itself is constructed of 3.4-mil Elgiloy ${ }^{2}$; a low density, high-tensile strength steel alloy of cobalt, chromium, and nickel (see Table 3.2). The alloy is non-magnetic and rustproof. The vacuum windows are affixed by 1 inch steel flanges with an inner diameter of 7.125 inch. The drift pipe has a slight sag in the middle. The drift pipe connects the decay region to an underground enclosure, MI-13, that houses the LMC detectors.

\subsubsection{Collimator}

Immediately downstream from the drift pipe is a steel and tungsten collimator to form a narrow aperture blocking muons that come from the dirt surrounding the drift pipe. The collimator is a $17 \times 17 \times 81$ cubic-inch solid steel box with $2 \times 2 \times 81$ cubic-inch tungsten core running the length in the middle. A hole runs the length of the tungsten core. The hole starts with a $0.6 \mathrm{~cm}$ diameter circle that increases by 0.0148 $\mathrm{cm}$ every 3 inches until it reaches a diameter of $1.0 \mathrm{~cm}$ at the downstream end. The collimator has an adjustable support structure allowing the position of each end to be adjusted separately. The collimator is positioned such that the center hole points back into the decay volume avoiding both flanges and the drift pipe's sag. The optimum angle was set by taking a series of data runs where the position and angle of the collimator

\footnotetext{
${ }^{2}$ Elgiloy was developed by the Elgin Watch company for use in watch springs.
} 
was adjusted, mapping out the edges of the drift pipe. Additionally, the position was adjusted to illuminate as much of the tracker as possible.

\subsubsection{Veto}

Mounted on the downstream face of the collimator are four overlapping scintillator paddles that surround the hole. Its purpose is to tag particles that passed through the edge of the collimator. These paddles are $1.25 \times 1.5 \times 0.25$ cubic-inch in dimension, with a $0.5 \mathrm{~cm}$ radius half circle cut out of the long end. Each scintillator is attached to a roughly 12 inch light guide that is attached to a PMT. Each scintillator is optically isolated from the others. The scintillators are affixed to a 0.25 inch thick aluminum plate $40 \times 40 \mathrm{~cm}^{2}$ in area. Two scintillators are attached such that their half circular cut-outs meet to form a $1.0 \mathrm{~cm}$ diameter circle that is centered on the collimator's hole. The top and bottom two scintillators are attached directly to the aluminum plate, with the right and left paddles sitting on top (downstream) with their hole aligned with the top and bottom hole and the collimator. Fig. 3.4 and Fig. 3.3 shows the veto mounted on the collimator. The veto constitutes the first active volume of the system with its four channels.

\subsubsection{Fiber Spectrometer}

The fiber spectrometer measures the momentum of a charge particle by measuring the deflection caused by a dipole magnetic field. There are 4 planes of scintillating fibers positioned upstream of the magnet; 2 planes are located downstream of the magnet. Each plane of fibers consists of two overlapping sub-planes, with the second plane offset by half a fiber diameter from the first sub-plane. This is to prevent tracks from slipping though the cracks. The scintillating fibers have an active region $1 \mathrm{~mm}$ in diameter with a $0.002 \mathrm{~mm}$ thick transparent cladding. The two sub-planes are separated by $1.296 \mathrm{~mm}$ in $z$ between the centers of the planes. All of the fibers are 1.5 inch long. The fiber 
Table 3.3: The position of the fiber planes relative to the center of the dipole magnet.

\begin{tabular}{ccc} 
Plane & $z$-position [inch] & \# of fibers \\
\hline \hline 1Y & -18.56 & 29 \\
1X & -18.37 & 29 \\
2Y & -6.88 & 29 \\
2X & -6.70 & 29 \\
3X & +6.70 & 99 \\
4X & +18.37 & 183
\end{tabular}

planes are attached to four aluminum stands. The pitch angle planes, or $y$-planes, are mounted on the upstream side of their respective bend angle planes. Table 3.3 lists the $z$ positions of the center of each fiber plane, which is the central gap between the subplanes of each plane. Additionally, the number of fibers in each plane (both sub-planes) is given. The overlapping area of the upstream planes at each position is $1.5 \times 1.5 \mathrm{~cm}^{2}$, larger than the $1.0 \mathrm{~cm}$ diameter hole through the collimator. It should be noted that the fibers are not explicitly optically isolated, though the total internal reflection of the fibers keeps the cross-talk to a minimum.

Custom aluminum connectors attach to one side of the of the scintillating fibers, aligning a single $1 \mathrm{~mm}$ non-scintillating fibers into direct contact with each scintillating fiber. Both the ends of the scintillating fibers, and the attached fibers are highly polished.

Instrumenting every fiber of each fiber plane would require 398 channels and was impractical for budgetary reasons. A compromise of 149 channels was chosen based on the expected momentum resolution due to geometric effects [76]. This required bundles of between 1 and 18 fibers depending on the fiber plane and position. Fiber bundles bunch fibers between the sub-planes of each plane, creating an instrumented area with no gaps. The edges of each bundle overlap the next bundle by half a fiber width. Fibers in planes $1 \mathrm{X}, 2 \mathrm{X}, 1 \mathrm{Y}$, and $2 \mathrm{Y}$ have at most three fibers in a bunch, and these tend toward 
the outer edges of the planes. The edges of plane $4 \mathrm{X}$ have 18 fibers per bunch. The edges of the fiber planes are most likely to detect the low momentum muons; the inner region detect the higher momentum muons. As the high momentum muons are from kaons, the tracker is instrumented to better measure those muons. Each fiber bunch is epoxied through a hole in a 0.75 inch acrylic "cookie" approximately $1 \mathrm{~cm}$ thick, and the end is highly polished.

The cookies are mated to 149 6-stage Hamamatsu R1666 0.75 inch PMTs [36] by optical grease. The PMTs are connected to custom bases. The PMT gain quoted by Hamamatu is $10^{5}$. Each base holds up to 4 PMTs. The PMTs are held at the same high voltage, split between the 7 dynodes. The anodes have individual powered preamps and post-amps for signal readout. The high-voltage and low-voltage grounds are isolated on the card. The pre-amps are OPA647U chips, and the post-amps are AD8014 chips. The additional gain provided by the op-amps is 400 . The op-amps are powered by $\pm 5 \mathrm{~V}$. Each PMT is read out by its own Lemo cable. The PMTs were pre-sorted based on the high voltage needed to plateau, with 4 similar PMTs placed on the same base with a single high voltage value applied. The high voltages varied from 800-1800 V per card.

\subsubsection{Dipole Magnet}

A uniform dipole magnet resides between planes $2 \mathrm{X}$ and $3 \mathrm{X}$. The magnet is $16 \times$ $17 \times 12$ cubic-inch with a $8 \times 1 \times 12$ cubic-inch air gap. A uniform dipole field of $0.2273 \mathrm{~T}$ is provided over a $8 \times 1 \times 9.5$ cubic-inch region in the middle of the air gap, constituting a momentum kick of $p_{\mathrm{T}}^{\text {kick }}=16.43 \mathrm{MeV} / c$. The field is uniform over a region of 9 inches, and decays quickly over half an inch on each side. The bulk of the magnet is composed of non-magnetized steel to act as a yoke to contain the field. The field is along the vertical, $y$, direction, causing charge particles traveling in the $z$ direction to curve in the $x-z$ plane. 
Table 3.4: The $x-y$ dimensions of each individual range stack layer in inches. All the scintillators are 0.375 inches thick. Layer 8 is comprised of two sub-layers; one aligned vertically, the other horizontally.

\begin{tabular}{cccc} 
Layer & $x$ & $y$ & Paddles per layer \\
\hline \hline 1 & 2.30 & 4.00 & 3 \\
2 & 2.53 & 7.00 & 3 \\
$3-8$ & 3.75 & 7.75 & 2
\end{tabular}

\subsubsection{Muon Acceptance Counters}

Immediately downstream of the fiber tracker is a secondary veto to accept clean tracks through the system that pass through plane 4X. The four scintillators that define this detector are referred to as the muon acceptance counters (MACC) and constitute two veto paddles, and two acceptance paddles. The acceptance paddles are located on beam right and beam left and are $2 \times 0.8 \times 0.375$ cubic-inch in dimension. Above and below these paddles sit the veto paddles, each are $6 \times 3 \times 0.375$ cubic-inch in dimension. The paddles are optically isolated. The MACC is mounted on the upstream side of the muon range stack.

\subsubsection{Muon Range Stack}

The muon range stack is designed to select muons from hadrons and electrons in the beam. It is composed of interleaving tungsten and scintillator planes. Two plates of $8 \times 8 \times 1$ cubic-inch plates are stacked to form the first seven tungsten layers. The eight layer is a single plate. The first two scintillator layers are segmented into 3 paddles; right, left, and center. Layers 3-7 are segmented into two paddles; right and left. The final layer is segmented into two layers of paddles forming right-left, and top-bottom layers. Table 3.4 lists the dimensions of the scintillator paddles used in each layer. 


\subsubsection{Booster and MiniBooNE Beam Extraction}

The proton beam incident on the MiniBooNE target is provided by the Fermilab booster [30] over a $1.6 \mu$ s proton beam spill. The booster beam micro-structure, or bunch, is determined by the $52.8 \mathrm{MHz}$ extraction rate. Each spill can contain up to 84 bunches, more often the number is around 81 . The proton bunches are on order a few ns wide and occur once every 18.9352 ns during the spill. However, the singles rate per channel in the LMC at ten turns is too high. Special one-turn booster runs were requested for the set of LMC data.

Upstream of the MiniBooNE target is a break in the beam pipe sealed with an insulator. A resistor is connected across the break. As the beam passes through the pipe, an image current runs along the beam pipe. At the point of the break this current is measured as a voltage across the resistor. This device is called the resistive wall monitor (RWM). The time of every hit in the LMC is measured relative to the time the beam passes the RWM.

\subsubsection{Electronics and DAQ}

Most of the LMC electronics are housed in a surface building (MI-13A) approximately 30 feet away from the LMC. The high voltage and signal cables are run through a duct from the surface building to the under enclosure entrance. The cables then descend roughly 30 feet vertically into the MI-13 pit. Both a confined space reclassification form and no beam extractions to MiniBooNE were required for entrance into the enclosure.

The LMC Data Acquisition electronics were triggered off the kicker magnet $(1 \mathrm{D} 1 \mathrm{~F})$ that extracted beam from the booster into the MiniBooNE beamline. Additionally, a signal from the RWM were required to time events.

The PMT signals lead into discriminators and then CAMAC TDCs. The TDCs are read-out once per spill and have a 16 hit buffer. If there were more than 16 hits 
in a channel, the early events are lost. TDC buffer overflow is the reason that normal intensity data in the LMC saturates, and too many hits cause the tracking algorithm to misreconstruct. Even at low intensity some of the channels would occasionally saturate. These TDC channels were backed up with an additional TDC channel that stopped accepting hits after 16 . This way, the overlapped hits between the two channels could be matched to accept up to 31 hits before having the possibility of lost hits. The TDCs and the DAQ were required to handle 177 channels.

\subsubsection{Calibration}

Events in the LMC detectors are localized into their respective bunches of a given spill by adjusting a time offset per PMT. This adjustment causes particles of average speed through the detector (in this case $\sim c$ ) to "hit" all planes at the same time. Fig. 3.5 shows the effect of the calibration algorithm on every PMT in the $1 \mathrm{X}$ plane. The shape of the peaks can differ based on the properties of the PMTs so the algorithm adjusts the relative placement of the bunches in the spills so that the minimums are roughly between -12 and -10 ns. Some PMTs get adjusted by eye in the event the algorithm was not successful. Fig. 3.6 shows the hit timing of a PMT after calibration. The bunch structure is clearly visible.

These calibrations allow for the forming of coincidences between events in the fiber tracker. The coincidences will be the basis for track finding and measurement of a charged particle's momentum.

\subsubsection{Simulation}

The MiniBooNE beam MC was built within the GEANT4 [41] MC framework. This MC acts as input to the LMC GEANT3 [56] based MC. The beam MC produces these inputs in two different configurations. The first configuration propagates pions and kaons to a certain distance down the decay pipe and records their momentum in a slice 


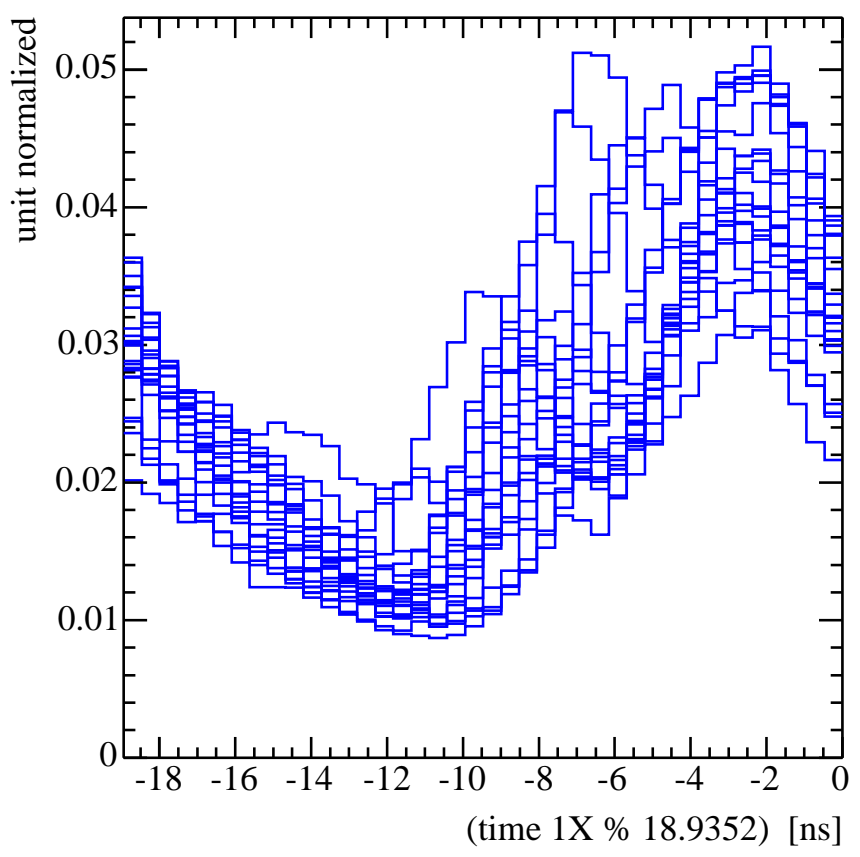

Figure 3.5: The timing of hits in fiber plane $1 \mathrm{X}$ separated by PMT modulo the bunch structure. The algorithm adjusts the last bunch to be less than 0 relative to the RWM time.

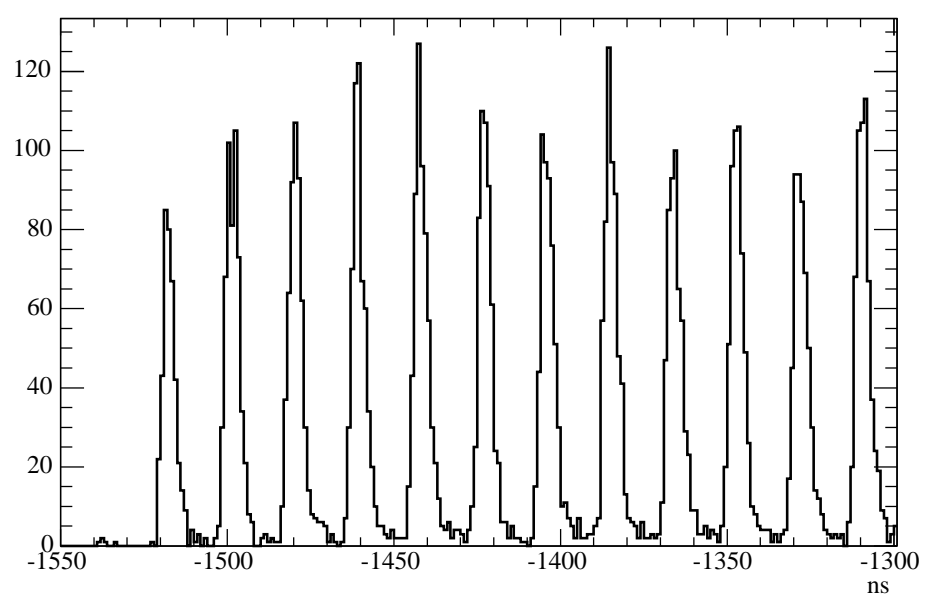

Figure 3.6: The timing structure of PMT \#48 in the fiber tracker at the beginning of the spill. The bunch structure is clearly visible. As only a few hits per PMT can be recorded during a single spill, a large sample of data is included in this plot. 
at that location. The LMC MC then handles the slice by forcing the decays of the pions and kaons and records where the decay products intersect with the decay pipe wall. If the decay pipe wall is hit around where the drift pipe intersects, the particles are kept. The pions and kaons, in the slice, are additionally decayed $10^{4}$ times isotropically in their rest-frames. The statistics are also boosted based on the azimuthal symmetry of the round decay pipe. Independent statistical samples allow up to 106 rotations of the beam before the section that intersects the beam pipe are repeated. These particles, from the 106 rotations, are then propagated down the drift pipe with interactions turned on. Once particles reach the front face of the collimator, the positions and momentum are transformed to force the particles into a region within $2 \mathrm{~cm}$ of the center of the collimator. The reason being that the momentum distribution of particles at a given point on the face of the collimator is roughly the same as any other point. These tricks must be performed to increase the statistics of tracks actually simulated through the detector. A sample of $10^{7}$ simulated protons is boosted by $10^{4} \pi / K$ decays, 106 rotations of the beam, and 1,111 by forcing tracks to near the collimator hole to yield the equivalent of $1.178 \times 10^{16}$ p.o.t., or about 3 hours running at one-turn intensity.

The second mode of running the beam MC for LMC studies lets GEANT4 handle the decays of pions and kaons and also interactions with the air in the decay pipe. However, this reduces the final MC statistics by $10^{4}$. Regardless of the mode that the beam MC used to produce the LMC MC input, the propagation of particles down the drift pipe and in the dirt is handled by GEANT3.

The careful design and measurements of the LMC's passive and active components detailed in $§ 3.2 .1$ are simulated in the GEANT3 LMC MC with a few minor caveats. The simulation does not simulate the PMTs, cables, non-scintillating fibers, support structures, the tracker's lid, or any of the electronics. The MC records whether an active volume is hit, by what particle, and that particle's momentum. Fig. 3.7 shows up close the GEANT3 renderings of a set of upstream fiber planes, the veto, and the 
muon filter with attached MACC. Additionally, the collimator and dipole magnet are
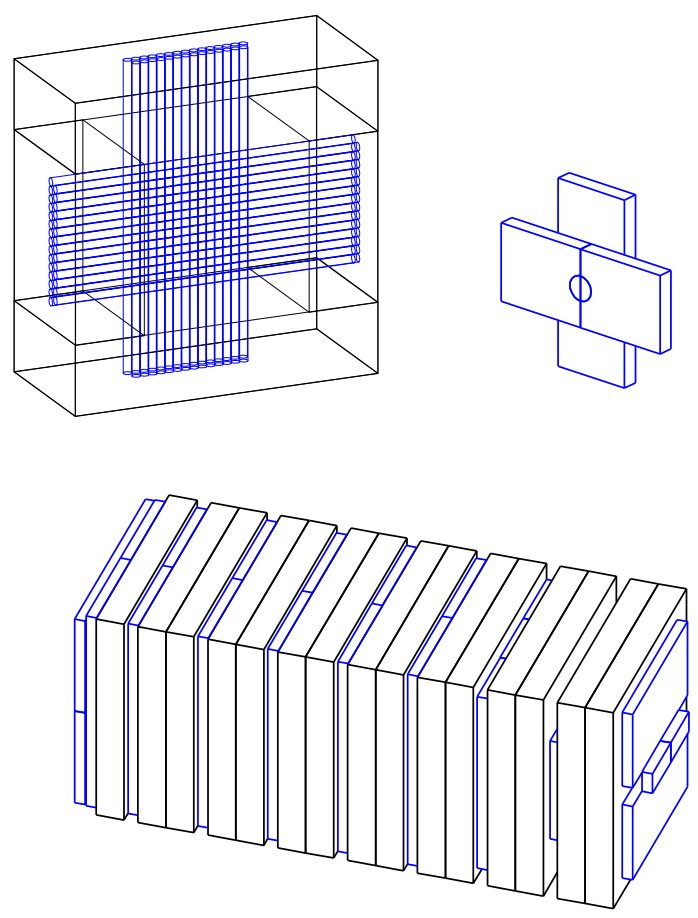

Figure 3.7: GEANT3 renderings of several of the LMC simulated components. Active volumes are blue. Upper Left: Fiber planes 1X and 1Y. Upper Right: The veto counters. Bottom: The muon filter. The exposed face shows the MACC.

simulated. Several non-standard material definitions are used to describe the LMC in the simulation. The "dirt" is simulated as concrete, the scintillator as Lucite. Table 3.5 shows the properties of several material definitions that were incorporated into the LMC MC. Fig. 3.8 shows the simulation of a gas of muons fired at the front face of the collimator. All simulated detector components are shown, along with the tracks of muons through those components. The bend caused by the dipole field is clearly visible. Penetration of the range stack requires a $1.3 \mathrm{GeV} / c$ momentum muon.

The simulation records the passage of particles into active volumes. As the actual detector has detection inefficiencies, the hits recorded by the $\mathrm{MC}$ may sometimes not occur. To mock up the effect of inefficiencies, each channel is ascribed a detection 
Table 3.5: Non-standard GEANT3 material definitions: the material, average atomic number, average number of protons, density, and radiation length are listed.

\begin{tabular}{lrrrc} 
Name & \multicolumn{1}{c}{$\langle A\rangle$} & \multicolumn{1}{c}{$\langle Z\rangle$} & $\rho\left[\mathrm{g} / \mathrm{cm}^{3}\right]$ & \multicolumn{1}{c}{$X_{0}\left[\mathrm{~g} / \mathrm{cm}^{2}\right]$} \\
\hline \hline Steel & 55.638 & 25.899 & 7.87 & 1.760 \\
Lucite & 12.399 & 6.236 & 1.18 & 33.644 \\
Concrete & 23.649 & 11.695 & 2.50 & 10.700 \\
Tungsten & 183.850 & 74.000 & 19.30 & 0.350 \\
Elgiloy & 59.541 & 27.410 & 6.35 & 2.156
\end{tabular}

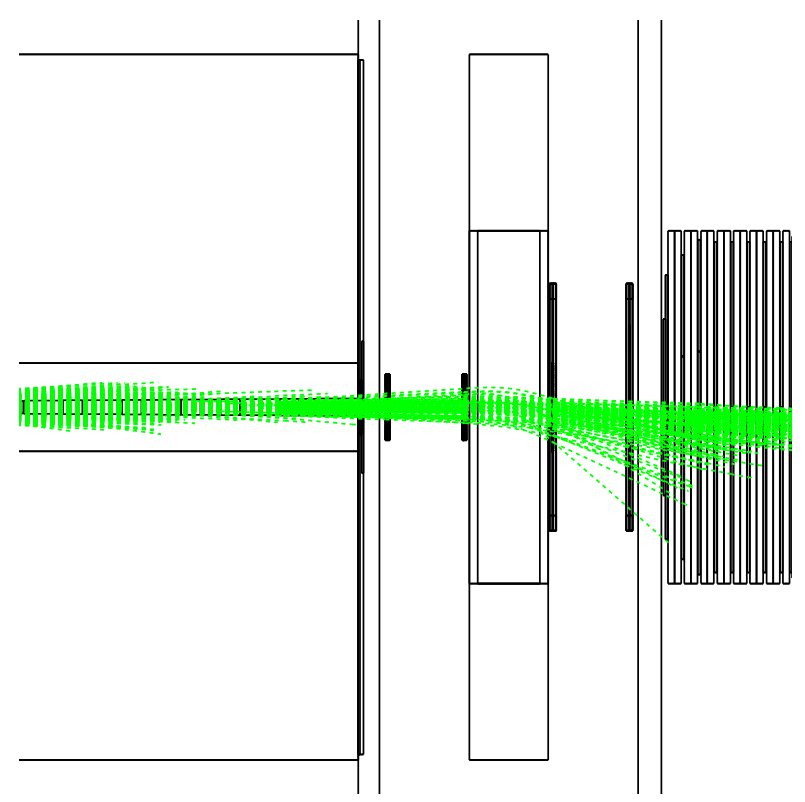

Figure 3.8: A simulation of a gas of muons incident the LMC collimator. Part of the collimator, the drift pipe, and part of the muon filter are not shown.

efficiency and each time a particle enters that volume, a random number is thrown against that efficiency to decide whether the volume was hit.

\subsection{Event Reconstruction}

Event reconstruction in the LMC is tasked with the reconstruction of charged particle momentum. The first task of the reconstruction is to group the hits in time to 
form coincidences. The calibration adjusted the hit times such that the bunch structure of each channel occurred at the same time. The coincidence finding groups hits within a $10 \mathrm{~ns}$ window. The window is large enough to select hits within the same bunch while rarely including hits from a different bunch. The coincidences require that at least one hit occurred in each fiber plane. Hits from the veto/MACC/filter are added to coincidences, but are not used for momentum reconstruction.

Once the coincidences have been formed, a simple algorithm fits tracks through the combination of hits in the fiber tracker, chooses the best fit track and estimates the track momentum. The coincidences also decide how far a particle penetrated into the muon filter. The penetration range is determined by the furthest layer hit, allowing for the absence of one layer due to inefficiency.

\subsubsection{Tracking Algorithm}

The problem is simplified by assuming that the full "kick" of the dipole magnet occurs at a plane that bisects the center of the magnet. The muon track through the hodoscope is then assumed to be that of two line segments, one for each the upstream and downstream segments, that intersect at the plane bisecting the center of the magnet. This is fully described by three parameters: the intersection, $x_{0}$; the upstream slope, $m_{\mathrm{u}}$; and the downstream slope, $m_{\mathrm{d}}$. The line segments are,

$$
\begin{aligned}
& x=m_{\mathrm{d}} z+x_{0} \\
& x=m_{\mathrm{u}} z+x_{0}
\end{aligned}
$$

where $x$ is the position in the bend plane, and $z$ is the beam direction. The upstream planes are $1 \mathrm{X}$ and $2 \mathrm{X}$ and measure the angle before the magnet. The downstream planes are $3 \mathrm{X}$ and $4 \mathrm{X}$ and measure the track after the bend.

The fibers in a plane are broken up into channels either by single fiber or a group of fibers. Regardless, the width of the fiber(s) are non-negligible and define an area 
that the track could have passed through. The position of a hit in a channel is set at the center of the fiber, or fiber bundle, and the uncertainty is set by the width of that fiber/bundle assuming that it forms a uniform distribution (width $/ \sqrt{12}$ ). Occasionally two adjacent channels will be hit because the fibers on the edges of the bundles overlap. The hit is then set at the center of the overlap area and the width is just of that overlap.

For a given set of track parameters, a goodness of fit $\chi^{2}$ can be formed and is given by,

$$
\chi^{2}=\sum_{u} \frac{\left(m_{\mathrm{u}} z_{u}^{\prime}+x_{0}-x_{u}^{\prime}\right)^{2}}{\sigma_{u}^{2}}+\sum_{d} \frac{\left(m_{\mathrm{d}} z_{d}^{\prime}+x_{0}-x_{d}^{\prime}\right)^{2}}{\sigma_{d}^{2}}
$$

where $u$ sums over the upstream planes, $d$ sums over the downstream planes, $\sigma_{i}$ is the uncertainty of the hit, and $m_{\mathrm{u}, \mathrm{d}}$ have Roman subscripts to distinguish them from the summation indices. The primes represent the measured values of the hits, the $z$ positions are fixed by the positions of each fiber plane. The $\chi^{2}$ can be minimized analytically by taking derivatives with respect to the track parameters. This leads to a system of equations,

$$
\left(\begin{array}{c}
\sum_{u} \frac{x_{u}^{\prime}}{\sigma_{u}^{2}}+\sum_{d} \frac{x_{d}^{\prime}}{\sigma_{d}^{2}} \\
\sum_{u} \frac{x_{u}^{\prime} z_{u}^{\prime}}{\sigma_{u}^{2}} \\
\sum_{d} \frac{x_{d}^{\prime} z_{d}^{\prime}}{\sigma_{d}^{2}}
\end{array}\right)=\left(\begin{array}{ccc}
\sum_{u} \frac{1}{\sigma_{u}^{2}}+\sum_{d} \frac{1}{\sigma_{d}^{2}} & \sum_{u} \frac{z_{u}^{\prime}}{\sigma_{u}^{2}} & \sum_{d} \frac{z_{d}^{\prime}}{\sigma_{d}^{2}} \\
\sum_{u} \frac{z_{u}^{\prime}}{\sigma_{u}^{2}} & \sum_{u} \frac{z_{u}^{\prime 2}}{\sigma_{u}^{2}} & 0 \\
\sum_{d} \frac{z_{d}^{\prime}}{\sigma_{d}^{2}} & 0 & \sum_{d} \frac{z_{d}^{\prime 2}}{\sigma_{d}^{2}}
\end{array}\right)\left(\begin{array}{c}
x_{0} \\
m_{\mathrm{u}} \\
m_{\mathrm{d}}
\end{array}\right)
$$

that are exactly solvable assuming the matrix has a determinant. The problem of track fitting has been reduced to the inversion of a $3 \times 3$ matrix, which is handled by the analysis code. For situations that have multiple hits in the fiber planes, the algorithm loops over all possible combinations and chooses the hits with the minimum $\chi^{2}$.

\subsubsection{Momentum Reconstruction}

The momentum of a charged particle can be estimated based on the reconstructed track parameters. A charged particle traveling in a uniform magnetic field will travel in 
a helix. According to the Lorentz force,

$$
\frac{d \mathbf{p}}{d t}=Z e(\mathbf{v} \times \mathbf{B})
$$

where $Z$ is the charge (in units of electron charge), $e$ is the electron charge, $\mathbf{v}$ is the particle's velocity, and $\mathbf{B}$ is the magnetic field. The initial momentum of the particle is given by [20],

$$
\left|\mathbf{p}_{0}\right| \cos \lambda=0.3 Z R B
$$

where the momentum is in $\mathrm{GeV} / c, R$ is the radius of curvature in meters, $\lambda$ is the pitch angle, and $B$ is the magnetic field magnitude in Tesla. The reconstructed track parameters estimate the radius of curvature as,

$$
R=z \csc \theta
$$

where $z$ is the width of the magnetic field, $\theta$ is the angle between the incoming and outgoing track, and $\alpha$ is the incoming track angle. In terms of the fit parameters,

$$
R=z \frac{\sqrt{\left(1+m_{\mathrm{d}}^{2}\right)\left(1+m_{\mathrm{u}}^{2}\right)}}{\left|m_{\mathrm{d}}-m_{\mathrm{u}}\right|}
$$

gives the radius of curvature.

To verify that tracks were being appropriately fit, an event display that shows the position of hit fibers and the reconstructed track parameters was produced. Fig. 3.9 shows two data events that are good muon candidate events. Additionally, with the $y$ planes the tracks can be projected back to the collimator to see where they came from. Fig. 3.10 shows the projection back to the front face of the collimator. The projection shows that the majority of hits came from the hole of the collimator.

\subsubsection{Fiber Plane Efficiencies}

The fiber plane efficiencies were determined in data and account for several effects: true hits falling just outside the coincidence window and cases where the passage of the 

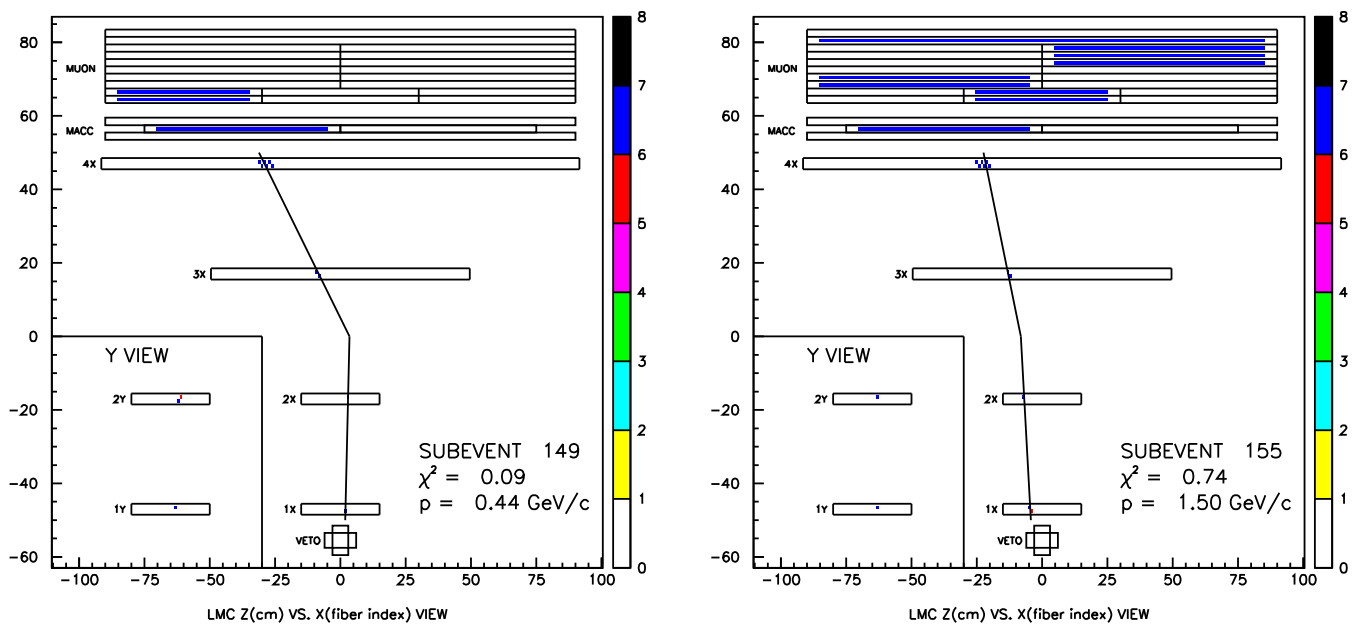

Figure 3.9: Two $\mu^{+}$candidate events. The $\mathrm{X}$ fiber planes are scaled to be closer than reality in this view. The reconstructed track parameters have been adjusted according. The veto, MACC, and muon filter are not to scale and are included to show hits in the active areas. The colors in a plane denote separate hits.
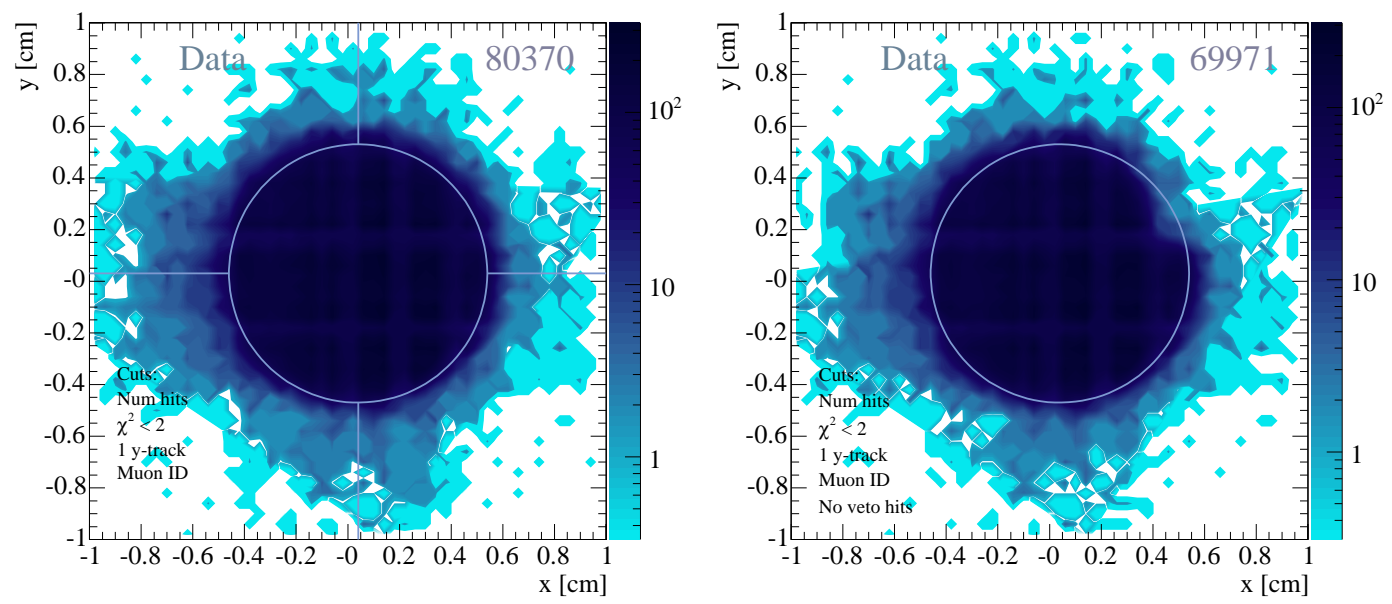

Figure 3.10: Projection of data events back to the front face of the collimator for well reconstructed $\mu^{+}$candidate events. Left: Any number of veto hits. Right: No veto hits. This shows the sensitivity to the fact that one of the veto counters was slightly in the beam.

charged particle failed to trigger the discriminators. The efficiencies were determined 
by forming a 5-plane coincidence and deciding a probability that a given channel was hit in the absent plane. The plane is then checked to see if it was indeed hit where expected. The efficiency calculation starts with the pseudo-efficiency given by,

$$
\epsilon_{i}^{\prime}=\frac{\sum_{n} h_{n}(i)}{\sum_{n} P_{n}(i)}
$$

where $h_{n}(i)$ is 1 if PMT $i$ was hit during coincidence $n, 0$ otherwise, and $P_{n}(i)$ is the probability that the given channel was hit. The probabilities are set by projecting the geometric area suggested by the 5 hit planes, and taking the overlap area with PMT $i$. To set the absolute efficiencies, the pseudo-efficiencies are measured in data and MC. The ratio of the data/MC pseudo-efficiencies is multiplied to the MC efficiency. The procedure is iterated a few times until the pseudo-efficiencies converge. The efficiencies were then applied to each channel of the fiber tracker simulations, adding the appropriate momentum smearing to the simulated distributions. The overall efficiency is $90-95 \%$ depending on the fiber plane.

\subsection{Analysis}

The goal of this analysis is to try an extract a constraint on the $K^{+}$normalization in the MiniBooNE secondary beam. First, the LMC signal MC needs to be propagated through the reconstruction with all interactions and detector inefficiencies applied. Fig. 3.11 shows these effects. This plot is the analog of the LMC concept in Fig. 3.2, presented here in momentum instead of energy. In a background free experiment, the $K$ parents would be reweighted in an attempt to match the measured data distribution. However, as the singles rate in data was many times larger than initially expected, is not expected to be a background free experiment.

The first task to removing backgrounds is to isolate a clean sample of well reconstructed events. This was done by requiring tracks with low reconstructed $\chi^{2}<2$, few hits per plane, no hits in the veto, tracks that point back into the collimator's hole, and 


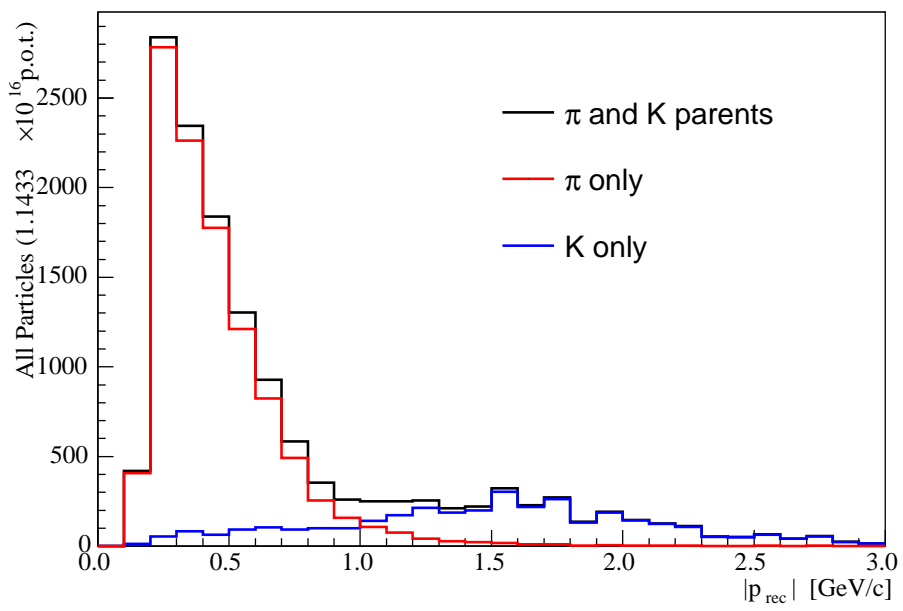

Figure 3.11: The reconstructed $\mu^{+}$momentum for $\pi^{+}$and $K$ parents based on the default beam MC. Resolution effects cause the distributions to smear into each other. Beam hadronic-interaction backgrounds are not included. Detector inefficiencies are included in the MC. This plot is the reconstruction analog of Fig. 3.2.

tracks that pass through only the active area of the MACC. By construction, virtually all MC events pass these cuts. Some do, however, get removed by the $\chi^{2}<2$ cut.

The next issue is to isolate a clean sample of muons from hadronic junk. The muon range stack is fully penetrated by a muon with $1.3 \mathrm{GeV} / c$ momentum; however, that is not necessarily the case for reconstructed momentum. Fig. 3.12 shows the reconstructed momenta vs. range stack penetration depth (range) for gases of muons and pions. The pions range out earlier than the muons. The muon-ID is set to keep $95 \%$ of the muons at a given range. A very small number of muons will be lost, but a significant fraction of hadronic junk will be cut. Fig. 3.13 shows the momentum vs. range for the data. A clear region of muons is identifiable within the muon-ID cut. Selecting the events that pass the muon-ID and quality cuts should provide a sample of events that are mostly muons, especially at large ranges. Fig. 3.14 shows the momentum distribution for data and signal MC for events that pass all of these cuts. An excess of data events is seen above the MC. These events are assumed to be mostly muons and implies a normalization difference of 1.7. The events that bulk up in the range $500-1500 \mathrm{MeV} / c$ are thought 

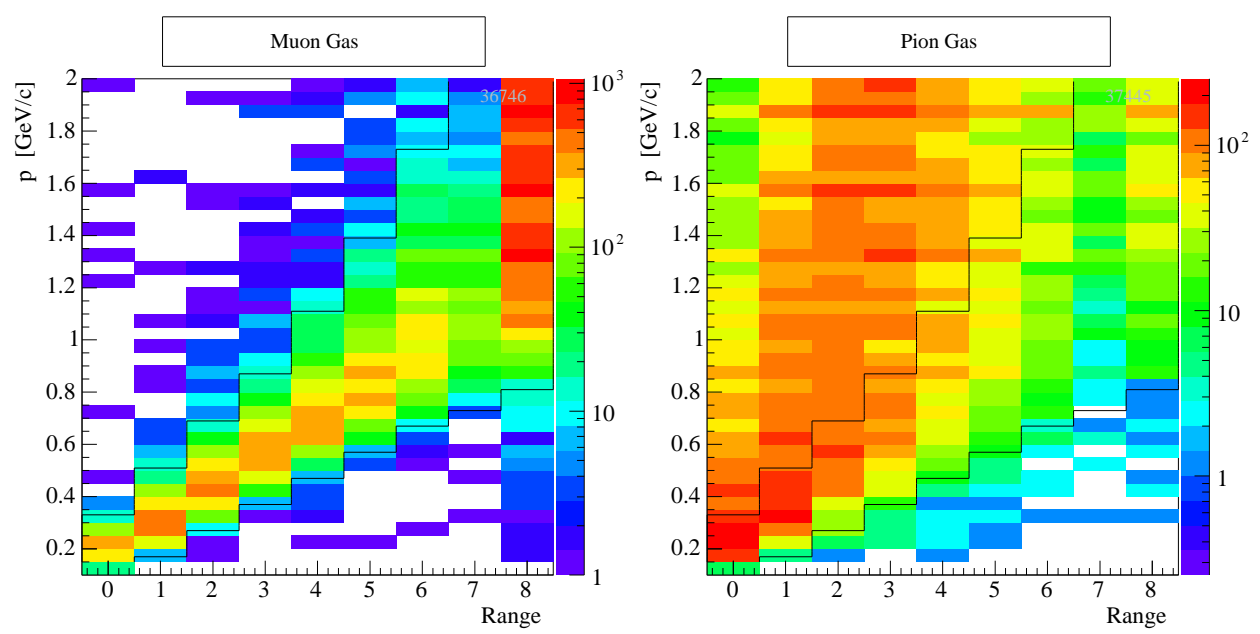

Figure 3.12: Left: Reconstructed momenta of a muon gas MC vs. penetration depth (range) into the range stack. The black lines define a cut to accept upwards of $95 \%$ of incident muons. All events between the lines are kept. Right: A pion gas MC over the same energy range as the muon gas. The pions range out much sooner than the muons.

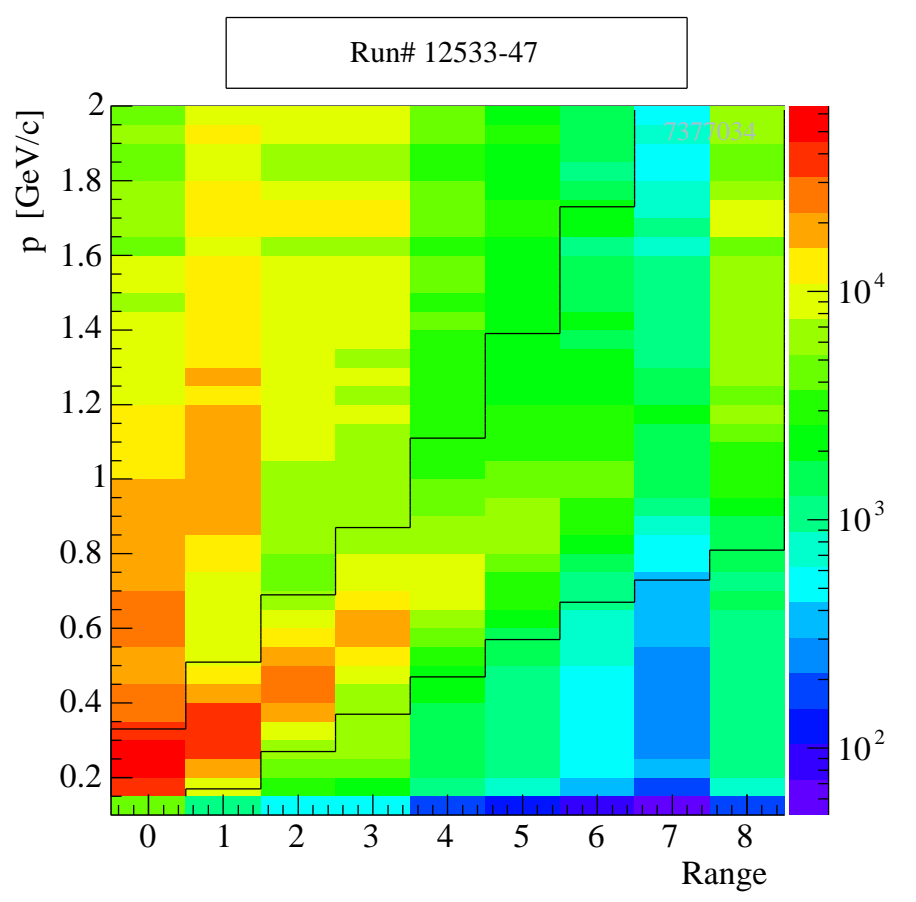

Figure 3.13: Reconstructed momentum vs. range for the LMC data set. The events separate into two clear regions; the muons within the muon-ID cut, and hadrons at high momentum and low range. 

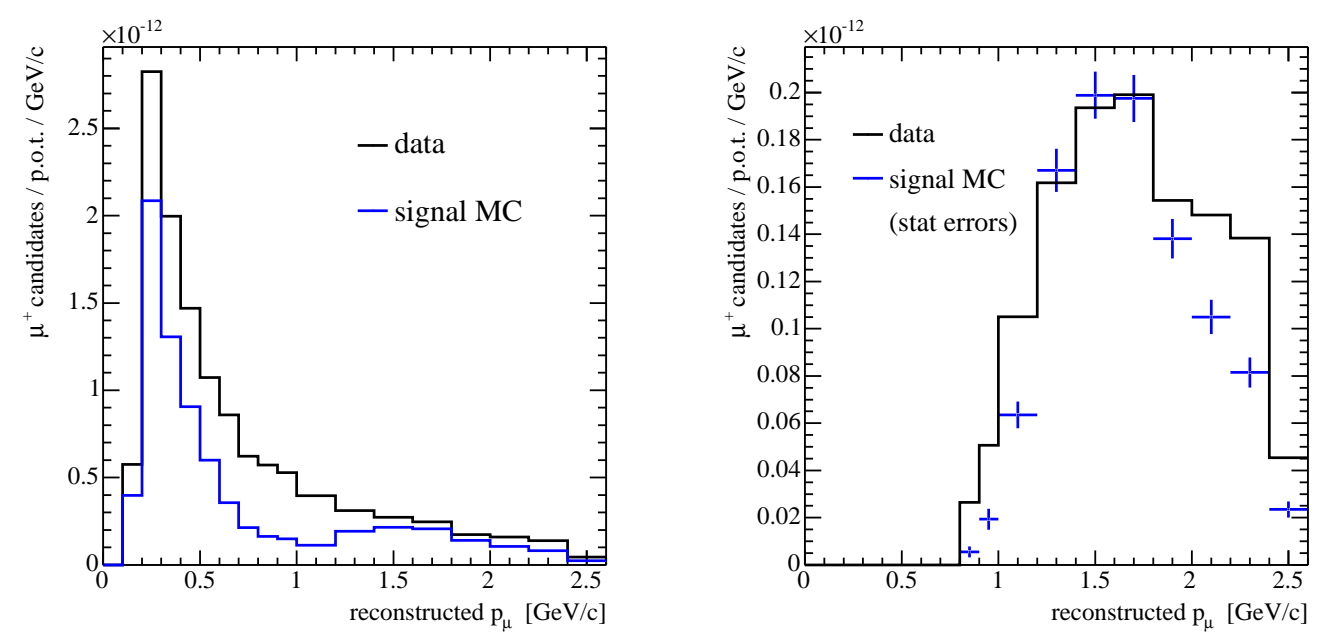

Figure 3.14: Left: The reconstructed momentum distribution for data and signal MC that pass all cuts. A significant difference is seen between data and MC (see text). Right: The reconstructed momentum distribution for data and signal MC after all cuts and restricted to range 8 in the muon filter. The errors are statistical on the MC. The data stats are insignificant in comparison.

to be muons that scattered through the dirt around the drift pipe but still were able to make it through the collimator and pass as signal. Restricting the range to muons that fully penetrated the range stack implies a normalization difference of 1.2. This can only be interpreted as an upper limit. Interestingly, the data distribution appears to be slightly broader than the MC. This is either due to unsimulated additional backgrounds, or worse momentum resolution in the detector than is simulated in the MC. Both of these effects are possible and could explain the fact that the MC is roughly equal to the data in the peak region, yet drops off substantially at low and high momenta. After all, slight differences in fiber position matter more for high momentum tracks than for low momentum tracks. The higher momentum tracks tend to pass through regions with less fibers, and relative differences in fiber bundle position between the data and MC would clearly cause differences in momentum resolution at high momentum. 


\subsection{Discussion}

What became clear during the course of this analysis was the need for a full simulation MC. Proton, pion, and kaon interactions in the air create additional muons that are incident on the LMC drift pipe. Additionally, interactions in the dirt can also create muons that can scatter into the collimator. All of these processes need to be simulated to get an accurate understanding of the muon related backgrounds. The trouble, was of course, computing resources. To fully simulate the necessary interactions required a MC that included a rather large total volume to track interactions. Doing so also limits the ability to boost statistics through clever tricks. The beam MC starts with single proton interactions. To simulate the number of protons that have actually struck the the target $\left(\sim 10^{21}\right.$ for MiniBooNE; $\sim 10^{17}$ for LMC data) requires enormous computing resources. It was estimated that it would take a couple of hundred cpus three of four months to generate enough statistics with a full background MC to match that taken by the data in the course of a few days. Perhaps, future experiments will have the resources to accomplish such a task. It was not deemed valuable enough to use that many resources for a possible kaon constraint.

In the end, this result interpreted as an upper limit to the possible amount of kaons in the beam, did not significantly improve the fits to external kaon production data. This result, when used as a cross check of the kaon fits, showed comparable precision. Kaon production off the target ended up not being a dominant source of uncertainty for the oscillation analysis. The reason was that the neutrino cross-section errors were unexpectedly large, limiting the usefulness of the LMC limit. Measuring a neutrino interaction cross-section is the subject to which this dissertation now turns. 


\section{Chapter 4}

\section{Event Reconstruction}

The purpose of event reconstruction is to identify particles and estimate their kinematic properties. Events are detected from the light they produce in the detector by photomultiplier-tubes (PMTs) on the detector's walls. Charged particles traveling in the MiniBooNE oil can produce two types of direct light: Čerenkov and scintillation light. If a charged particle is traveling faster than the local speed of light in a medium, it will emit Cerenkov radiation ${ }^{1}$. Since the speed of light in Marcol 7 mineral oil is approximately $19.5 \mathrm{~cm} / \mathrm{ns}$, a particle with a moderate boost, $\gamma>1.32(T>0.32 \times$ mass $)$, will produce Cerenkov light. This light is emitted azimuthally about the track direction at an angle related to the particle's velocity given by

$$
\cos \theta=\frac{1}{n \beta}
$$

where $\theta$ is relative to the trajectory, $n$ is the index of refraction, and $\beta$ is the particle's velocity. Combined with a hypothesis of the type of particle, this can directly measure the particle's energy. Clearly, as the particle loses energy the angle gets narrower and the amount of produced Čerenkov light decreases. Čerenkov light is emitted promptly and in the direction the particle is traveling. In the spherical MiniBooNE tank, Čerenkov light produces rings on the detector's wall.

Scintillation is produced as a result of ionization of the medium. Since the light is emitted as the electrons recombine with the ionized atoms, the light is slightly de-

\footnotetext{
${ }^{1}$ See for instance, the derivation in $\S 2.1 .4 .1$.
} 
layed relative to the Čerenkov radiation. The light produced from the recombination is occasionally due to the electron recombination falling to a state slightly above the ground state, a state not typically occupied. Otherwise, the light produced from the ionization would ionize nearby atoms and never leave the medium. Also, scintillation light is produced isotropically making it extremely difficult to measure track trajectories solely by scintillation.

As the direct sources of light propagate through the oil, they are subjected to fluorescence and scattering. This can cause the light to lose energy, change direction, delay, or get absorbed. The details of the propagation of light in the mineral oil are discussed in $\S 2.1 .4 .1$.

\subsection{Single-Track Reconstruction}

MiniBooNE uses a maximum likelihood method for event reconstruction to estimate the kinematic properties of particles in the detector [77]. This event reconstruction parametrizes the propagation of the light emitted by a charged particle, through interactions with the medium, to generate probability density functions (PDFs) for both the hit time (relative to the track start time) and total charge deposited to each PMT. The track itself is parametrized as a set of 7 variables:

- Event vertex $(x, y, z)$

- Event time at vertex $(t)$

- Track direction $(\theta, \phi)$

- Kinetic energy $(E)^{2}$

The set of parameters that describe an event will be referred to as the vector $\mathbf{u}$. However, the absolute event time, $t$, is not used for reconstruction, it is only used for a beam window cut. Only the relative time to the PMTs are used.

\footnotetext{
${ }^{2} E$ will refer to the kinetic energy in this section unless otherwise specified.
} 
Each type of particle in the detector generates a different distribution of light for the same track parameters. A detailed Monte Carlo (MC) was generated for each track hypothesis and used to parametrize the light profiles. This allows for the same fitter to be run for different particle hypotheses whose likelihoods allow for the identification of the particle type.

\subsubsection{Event Topologies}

Charged particles are distinguished by their Čerenkov light profiles. Each type of particle interacts with the detector depending on its mass and types of allowable interactions. The topological distinctions are discussed for the types of particles currently considered by the event reconstructions. Fig. 4.1 shows the kinetic energy of

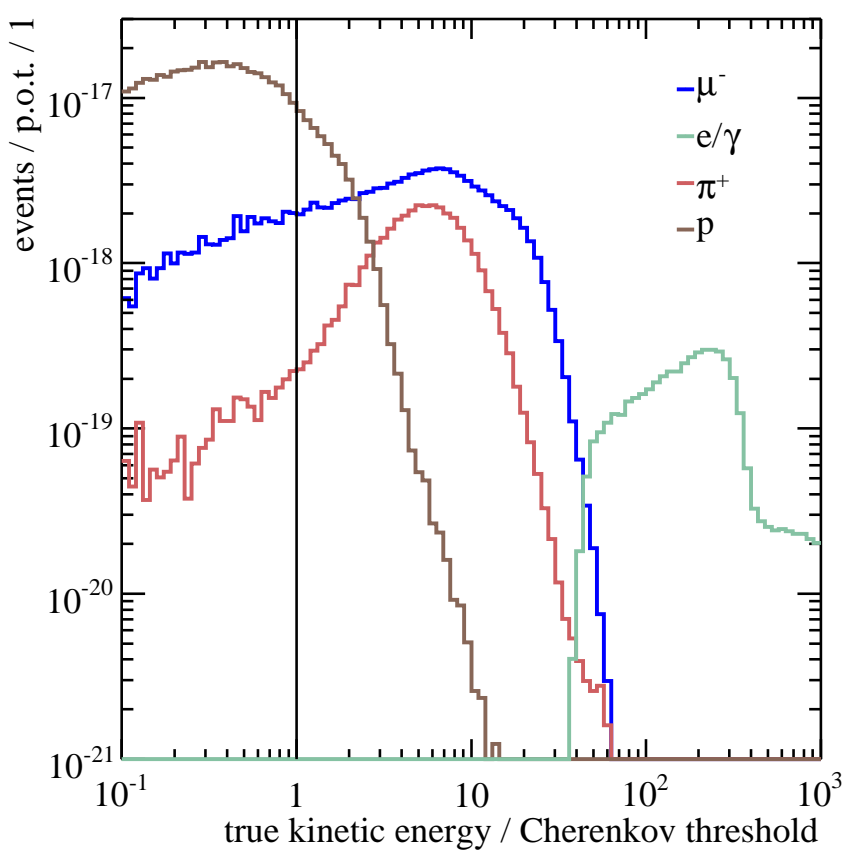

Figure 4.1: The MC true kinetic energy distributions for charged particles $\left(\mu, e / \gamma, \pi^{+}, p\right)$ weighted by their Cerenkov thresholds in mineral oil for events that make it into the $\mathrm{CC} \pi^{0}$ sample (except for $\pi^{+}$which come from $\mathrm{CC} \pi^{+}$events). Events to the right of the black line are above the Cerenkov threshold. The Cerenkov threshold for a charge particle in the mineral oil is approximately $0.32 \times$ mass. 
different particles weighted by their Čerenkov threshold. Every type of charged particle considered, except protons, is almost always above threshold.

\subsubsection{Muons}

A muon will produce Čerenkov radiation if it has a small amount kinetic energy $(E>33.6 \mathrm{MeV})$. Most muons in the MiniBooNE tank are above this threshold (see Fig. 4.1). Since muons are minimum ionizing at these energies, the Čerenkov ring tends to be a sharp, filled-in ring (see Fig. 4.2). Muons tend to deposit all of their energy before decaying at rest. The charges of the muon are indistinguishable except for the fact that $\mu^{-}$can capture, effectively reducing the lifetime compared with $\mu^{+}$.

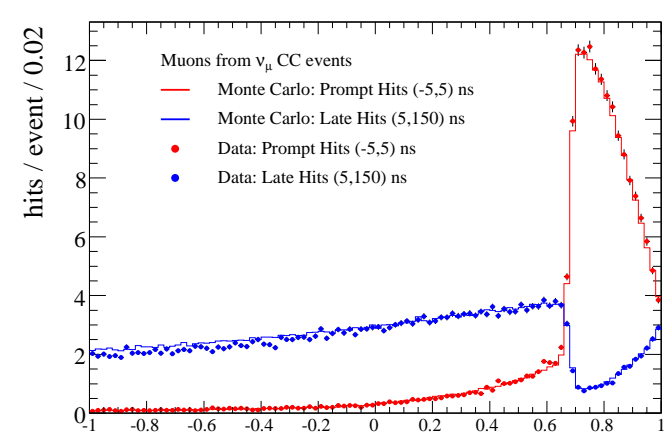

$\cos \theta$

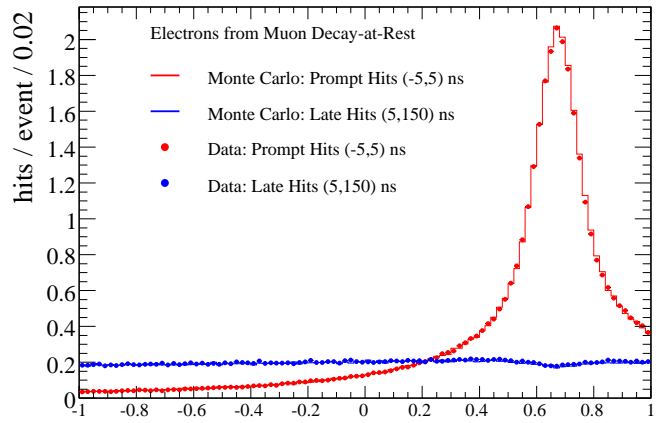

$\cos \theta$

Figure 4.2: The angular profiles for PMT hits relative to the track directions for both muons (left) and electrons (right) for prompt (red) and late (blue) light for both data and MC. The muons have energies between 400 and $500 \mathrm{MeV}$ and the electrons are from stopped muon decays. The muon's angular distribution shows a sharp edge (at $\cos \theta \sim 0.7)$ of the Cerenkov ring as opposed to the electron's fuzzier edge. The apparent dip in the late light distributions are due to the dead time of the electronics.

\subsubsection{Electrons}

All electrons produce Čerenkov light as it takes only a few MeV of kinetic energy to guarantee more than a few PMT hits. Fig. 4.1 shows electrons from photons and from stopped muon decays, even the lowest energy electrons are more than a factor of 10 
above threshold. Electrons interact more directly with the medium than muons do by producing electromagnetic showers and through multiple Coulomb scattering. Fig. 4.2 shows that the net effect is a track that scatters often, producing a "fuzzy" Cerenkov ring. These rings can get fairly dim in the centers, because even though the electron is still above threshold, it is not producing enough light to fill in the ring.

\subsubsection{Photons}

Photons travel some distance in the medium before they produce electromagnetic showers, initiated by pair-production that are indistinguishable from electrons. The photon conversion length in mineral oil is approximately $67 \mathrm{~cm}$ [77]. Fig. 4.1 shows photons weighted by the electron threshold, implying that even after they convert the electrons they produce are always over threshold.

\subsubsection{Neutral Pions}

The $\pi^{0}$ decays into $\gamma \gamma 98.8 \%$ of the time. The next largest decay mode is the $\gamma e^{+} e^{-}$Dalitz decay $(1.2 \%)$. In the rest frame, the two photons are produced back to back with energies equal to half the pion mass $(E=67.5 \mathrm{MeV})$. However, in the labframe of the detector, the photons can have drastically different energies. Also, since $c \tau=25.1 \mathrm{~nm}$ for the $\pi^{0}$, the decay vertex is effectively the event vertex for all $\pi^{0}$ decays in the detector. Because of the photon conversion length, the two photons tend to have disjoint vertices with both tracks "pointing" back toward the event vertex.

\subsubsection{Charged Pions}

A $\pi^{+}$interacts similarly to a muon except that it can have "hard" hadronic scatters. This implies that the track can have a sudden change in direction with a loss of energy between the upstream and downstream tracks. The resulting Cerenkov ring looks like a muon ring with a cut out middle, where the middle portion of the 
ring is displaced relative to the larger ring. Fig. 4.1 shows that most $\pi^{+}$are above the Čerenkov threshold. The $\pi^{-}$capture on nuclei and look very similar, however, they are not included as part of this reconstruction.

\subsubsection{Protons}

The energy threshold for protons to produce Čerenkov radiation is $300 \mathrm{MeV}$. Fig. 4.1 shows that most protons liberated from a nucleus are below this threshold. Protons also travel relatively short distances making their Cerenkov rings rather thin and diffuse. For the most part, direction information is lost for protons. Protons are not fit by this reconstruction ${ }^{3}$; a dedicated fitting technique is described in Ref. [74].

\subsubsection{Single-Track Likelihoods}

The main portion of the MiniBooNE tank contains 1280 PMTs. Each PMT is read out through a discriminator that can only trigger once every $200 \mathrm{~ns}$. The PMT's charge is integrated over that $200 \mathrm{~ns}$ window. A charged particle traversing the oil can easily travel up to a few meters, coming to rest roughly 10 ns after the initial neutrino interaction takes place. Light can take $50 \mathrm{~ns}$ to traverse the tank between the furthest points. The delayed light is produced with a roughly $30 \mathrm{~ns}$ exponential decay constant. All of the light production and propagation falls within the $200 \mathrm{~ns}$ window. This implies that the total charge per PMT, for a given event, is the sum of both the charge induced from the prompt and late light. For each PMT, the total charge and hit time are recorded by the data acquisition system. Therefore, for each event, there are 2,560 independent pieces of information.

For each set of track parameters, $\mathbf{u}$, for an assumed particle type, a set of probability distribution functions (PDFs) for the total charges and hit times $\left(f_{q}\left(q_{i} ; \mathbf{u}\right)\right.$ and $f_{t}\left(t_{i} ; \mathbf{u}\right)$ respectively, where $q_{i}$ and $t_{i}$ are the charges and times) across all PMTs are

\footnotetext{
${ }^{3}$ In principle, one could add a proton hypothesis to the fitter.
} 
calculated from predetermined tables. In general, the likelihood can be constructed as,

$$
\mathcal{L}(\mathbf{q}, \mathbf{t} ; \mathbf{u})=\prod_{i}^{N_{\text {unhit }}} \mathcal{P}_{i}(\text { unhit } ; \mathbf{u}) \times \prod_{j}^{N_{\text {hit }}} \mathcal{P}_{j}(\text { hit } ; \mathbf{u}) f_{q}\left(q_{j} ; \mathbf{u}\right) f_{t}\left(t_{j} ; \mathbf{u}\right)
$$

where $i$ and $j$ index over PMTs, $N_{(\text {un)hit }}$ are the number of PMTs (un)hit, and $\mathcal{P}_{i}(($ un)hit; $\mathbf{u})$ are the probabilities that a given tube was (un)hit for a given $\mathbf{u}$. The reconstruction is tasked with finding the set, $\mathbf{u}_{0}$, that maximizes this likelihood.

Finding the maximum likelihood is somewhat simplified in practice by separating the charge and time portions of the likelihood by defining:

$$
\begin{aligned}
-\log \mathcal{L}(\mathbf{u}) \equiv & F_{q}(\mathbf{u})+F_{t}(\mathbf{u}) \\
F_{q}(\mathbf{u})= & -\sum_{i}^{N_{\text {unhit }}} \log \mathcal{P}_{i}(\text { unhit; } \mathbf{u}) \\
& -\sum_{i}^{N_{\text {hit }}} \log \mathcal{P}_{i}(\text { hit } ; \mathbf{u})-\sum_{i}^{N_{\text {hit }}} \log f_{q}\left(q_{i} ; \mathbf{u}\right) \\
F_{t}(\mathbf{u})= & -\sum_{i}^{N_{\text {hit }}} \log f_{t}\left(t_{i} ; \mathbf{u}\right)
\end{aligned}
$$

where the probability that a tube was (un)hit is lumped in with the charge component. For simplicity, $F_{q}$ and $F_{t}$ are referred to as the charge and time "likelihoods" respectively, even though they are the negative logarithms of the likelihoods.

\subsubsection{The Charge Likelihood}

For a given PMT, one can assume that $f_{q}\left(q_{i} ; \mathbf{u}\right)$ is fully specified if one knows the number of observed photoelectrons, $n_{i}$, without any reference to other properties of the detector. Furthermore, $n_{i}$ is assumed to be a Poisson variable with mean $\mu_{i}(\mathbf{u})$ (called the predicted charge) which is explicitly dependent on the track parameters. This realization allows for the mapping of $\mathbf{u} \mapsto\left\{\mu_{i}\right\}$, where $\left\{\mu_{i}\right\}$ is the full set of predicted charges for all PMTs (the dependence on $\mathbf{u}$ is implicit). Therefore, the Poisson probability to not record a hit is given by,

$$
\mathcal{P}_{i}\left(\text { unhit } ; \mu_{i}\right) \equiv \overline{\mathcal{P}}_{i}\left(\mu_{i}\right)=e^{-\mu_{i}}
$$


with the probability of a hit given by,

$$
\mathcal{P}_{i}\left(\text { hit; } \mu_{i}\right) \equiv \mathcal{P}_{i}\left(\mu_{i}\right)=1-\overline{\mathcal{P}}_{i}\left(\mu_{i}\right)
$$

The predicted charge is separated into two parts: prompt and late components. The prompt predicted charge, $\mu_{\mathrm{prompt}, i}$, is due entirely to Čerenkov light. The late portion, $\mu_{\text {late }, i}$, is dominated by the direct scintillation light but also has contributions from indirect Čerenkov light, a slight contribution from direct Čerenkov light (from late pulsing), and also contributions from indirect scintillation light. The indirect light sources considered are from reflections, Raman and Rayleigh scattering, and fluorescence. The predicted charges, in terms of their Čerenkov and scintillation components, are

$$
\begin{aligned}
\mu_{i} & \equiv \mu_{\mathrm{prompt}, i}+\mu_{\mathrm{late}, i} \\
\mu_{\text {prompt }, i} & =0.95 \mu_{\mathrm{Che}, i}^{\text {direct }} \\
\mu_{\text {late }, i} & =0.05 \mu_{\mathrm{Che}, i}^{\text {direct }}+\mu_{\mathrm{Che}, i}^{\text {indirect }}+\mu_{\mathrm{sci}, i}^{\text {direct }}+\mu_{\mathrm{sci}, i}^{\text {indirect }}
\end{aligned}
$$

where the tabulation of each component and $f_{q}\left(q_{i} ; \mathbf{u}\right)$ are detailed in Refs. [77, 78] for muons and electrons, and Ref. [71] for $\pi^{+}$particles.

\subsubsection{The Time Likelihood}

The task of calculating the time likelihood requires finding the PDFs $f_{t}\left(t_{i} ; \mathbf{u}\right)$. Whereas the charge likelihood could be greatly simplified because the charge is an integrated quantity, the same is not true for the time likelihoods. The time of the hit is due to the first photoelectron to fire the PMT and is independent of all later photoelectrons. Therefore, $f_{t}\left(t_{i} ; \mathbf{u}\right)$ has a stricter dependence on $\mathbf{u}$ than $f_{q}\left(q_{i} ; \mathbf{u}\right)$ does. Some of the dependence on $\mathbf{u}$ can be reduced by introducing the corrected time as,

$$
t_{\mathrm{cor}, i}=t_{i}-t_{0}-\frac{r_{\mathrm{mid}, i}\left(E_{0}\right)}{c_{n}}-\frac{\Delta s_{\mathrm{mid}}\left(E_{0}\right)}{c}
$$

where $t_{i}$ is the measured PMT time, $t_{0}$ is the track start time, $\Delta s_{\text {mid }}\left(E_{0}\right)$ is the distance from the start point of the track to the mean Čerenkov emission point along the track, 
$r_{\text {mid }, i}\left(E_{0}\right)$ is the track energy dependent distance from the mean Čerenkov emission point of the track to the PMT, and $c_{n}$ and $c$ are the speeds of light in mineral oil and vacuum respectively. This simplification has reduced the PDF dependence to a five dimensional space, removing the event time and distance dependence to the PMT from the track. The corrected time shifts the event time to the minimum time that a photon from the mean Cerenkov emission point can reach the PMT. However, a few more simplifying assumptions are made so that the tabulation of the PDF is more practical. After mapping $t_{i} \mapsto t_{\mathrm{cor}, i}$ in the PDF, the assumption is made that the remaining dependence on $\mathbf{u}$ is due only to $E_{0}, \mu_{\text {prompt }, i}$, and $\mu_{\text {late }, i}$.

The time likelihood PDF is separated into separate primitive distributions for prompt and late light, and then combined to form the full PDF. The primitive distributions, $G_{\mathrm{prompt}, i}\left(t_{\mathrm{cor}, i} ; \mu_{\mathrm{prompt}, i}, E_{0}\right)$ and $G_{\mathrm{late}, i}\left(t_{\mathrm{cor}, i} ; \mu_{\mathrm{late}, i}, E_{0}\right)$ are tabulated in detail in Refs. $[77,78,71]$. These primitive distributions are combined by assuming that if a PMT is hit by a prompt photoelectron, the PDF should follow the prompt primitive distribution regardless of whether the PMT is hit by late photoelectrons. Therefore, the Poisson probability of a PMT hit given a prompt photoelectron ${ }^{4}$ is,

$$
w_{\text {prompt }, i} \equiv \mathcal{P}_{i}(\text { prompt } \mid \text { hit })=\frac{1-e^{-\mu_{\text {prompt }, i}}}{1-e^{-\mu_{\text {prompt }, i}} e^{-\mu_{\text {late }, i}}} .
$$

The Poisson probability for a late hit is,

$$
w_{\text {late }, i}=\mathcal{P}_{i}(\text { late } \mid \text { hit }) \overline{\mathcal{P}}_{\text {prompt }, i}=1-w_{\text {prompt }, i}
$$

by conservation of probability. The weights sum to unity because these are the probabilities that a tube was hit by a photon assuming that it had already been hit. If the probability had started with the late light, the expressions would be reversed and the assumption would have been that time runs backwards! The final time PDF is given by,

$$
f_{t}\left(t_{\mathrm{cor}, i} ; \mu_{\mathrm{prompt}, i}, \mu_{\text {late }, i}, E_{0}\right)=w_{\text {prompt }, i} G_{\text {prompt }, i}+w_{\text {late }, i} G_{\text {late }, i}
$$

\footnotetext{
${ }^{4}$ This is just a simple application of Bayes' theorem with the realization that $\mathcal{P}_{i}($ hit $\mid$ prompt $)=1$.
} 
where the primitives are tabulated in advance.

\section{2 $\mathrm{CC} \pi^{0}$ Reconstruction}

The beauty of the one-track reconstruction method is that it lends itself well to scaling. The likelihood function in Eqn. 4.2, is completely general for any number of tracks. The goal is to find a simple way to combine the PDFs and primitive distributions for multiple tracks such that the fundamental light profiles for a single track type can be utilized.

This fitting technique was originally developed to reject $\mathrm{NC} \pi^{0}$ events with very high efficiency to an electron neutrino appearance search. The details of that reconstruction are found in Refs. [77, 78], along with a measurements of the $\mathrm{NC} \pi^{0}$ differential cross-sections in Ref. [75]. A combination of the principles that went into the single-track and two-track fitters are extended to meet the needs for the $\mathrm{CC} \pi^{0}$ reconstruction.

\subsubsection{Event Topology}

A $\mathrm{CC} \pi^{0}$ is a neutrino-neutron interaction that produces a muon and neutral pion in the final state $\left(\nu_{\mu} n \rightarrow \mu^{-} \pi^{0} p\right)$. Because of the fast $\pi^{0}$ decay $\left(\tau \simeq 10^{-17} \mathrm{~s}\right)$, the $\mathrm{CC} \pi^{0}$ final state consists of a muon, two photons, and a proton that originate from a common event vertex. As protons are rarely above threshold (see Fig. 4.1), these events tend to contain three Čerenkov rings. Often, these rings overlap, causing PMTs to have charge contributions from multiple tracks.

\subsubsection{Three-Track Likelihood}

Every type of event reconstruction (CCQE, $\mathrm{NC} \pi^{0}, \mathrm{CC} \pi^{+}$) performed using this fitting philosophy prior to, and including this analysis $\left(\mathrm{CC} \pi^{0}\right)$, has assumed that the outgoing nucleon can be ignored. The CCQE fitter reconstructs only the lepton ( $e$ or $\mu$ ), the $\mathrm{NC} \pi^{0}$ fitter reconstructs the two photons from the $\pi^{0}$ decay, and the $\mathrm{CC} \pi^{+}$ 
fitter reconstructs the $\mu$ and the $\pi^{+}$. Justification for this assumption will be addressed in $§ 4.4$. As such, to fit a $\mathrm{CC} \pi^{0}$ event, only the muon and two photons in the final state must be reconstructed.

A general three-track likelihood, from a common vertex, has the following components:

- Event vertex $(x, y, z)$

- Event time at vertex $(t)$

- First particle's type $\left(I_{1}\right)$

- First particle's kinematics $\left(E_{1}, \theta_{1}, \phi_{1}, s_{1}\right)$

- Second particle's type $\left(I_{2}\right)$

- Second particle's kinematics $\left(E_{2}, \theta_{2}, \phi_{2}, s_{2}\right)$

- Third particle's type $\left(I_{3}\right)$

- Third particle's kinematics $\left(E_{3}, \theta_{3}, \phi_{3}, s_{3}\right)$

not including the track-types (those are chosen prior to the fit), there are 16 parameters that must be fit. For a $\mathrm{CC} \pi^{0}$ event, there are 15 parameters as the muon has no conversion length. The likelihood is constructed to be fairly generic, which will be exploited during the course of the seeding procedure. The set of parameters for all three tracks will also be referred to as $\mathbf{u}$, though for the most part, each track is treated independently. The set that maximizes Eqn. 4.2 is $\mathbf{u}_{0}$. Constructing the three-track likelihood for generic particles is addressed in the next two sections. 


\subsubsection{Charge Likelihood}

The charge likelihoods are extremely simple. The predicted charges for each track (both prompt and late) are calculated separately then added together to give,

$$
\mu_{\mathrm{total}, i}=\mu_{1, i}+\mu_{2, i}+\mu_{3, i}
$$

where the subscripts $(1,2,3)$ denotes each track. The three-track charge likelihood is then constructed exactly as the one-track case in $\S 4.1 .2 .1$, with the total predicted charge replacing the one-track predicted charge.

\subsubsection{Time Likelihood}

The time likelihoods are a bit trickier as the proximity of a track to a PMT needs to be included in the primitive distribution weighting. This is to keep track of the most probable photoelectron to trigger the PMT. The first step is to calculate all the primitives $G_{\text {light }, i}^{\text {track }}$, where "light" is "prompt" or "late," and "track" is denoted by the labels "near," "mid," and "far" sorting the tracks by $r_{\text {mid }, i}$ from smallest to largest. Given the predicted charges, the Poisson probabilities of no hit are,

$$
\begin{aligned}
& \overline{\mathcal{P}}_{\text {prompt }, i}^{\text {near }}=e^{-\mu_{\text {prompt }, i}^{\text {near }}} \\
& \overline{\mathcal{P}}_{\text {prompt }, i}^{\text {mid }}=e^{-\mu_{\text {prompt }, i}^{\text {mid }}} \\
& \overline{\mathcal{P}}_{\text {prompt }, i}^{\text {far }}=e^{-\mu_{\text {prompt }, i}^{\mathrm{far}}} \\
& \overline{\mathcal{P}}_{\text {late }, i}=e^{-\mu_{\text {late }, i}}
\end{aligned}
$$

with

$$
\mu_{\mathrm{late}, i}=\mu_{\mathrm{tot}, i}-\mu_{\mathrm{prompt}, i}^{\mathrm{near}}-\mu_{\mathrm{prompt}, i}^{\mathrm{mid}}-\mu_{\mathrm{prompt}, i}^{\mathrm{far}}
$$

where all the tracks contribute equally for the late predicted charge. The Poisson probabilities of one or more hits is given by $\mathcal{P}=1-\overline{\mathcal{P}}$. With another application of 
Bayes' theorem and the conservation of probability the weights are,

$$
\begin{aligned}
& w_{\text {prompt }, i}^{\text {near }}=\mathcal{P}_{i}(\text { prompt } ; \text { near } \mid \text { hit }) \\
& =\frac{1-e^{-\mu_{\mathrm{prompt}, i}^{\text {near }}}}{1-e^{-\mu_{\mathrm{prompt}, i}^{\text {near }}} e^{-\mu_{\mathrm{prompt}, i}^{\text {mid }}} e^{-\mu_{\mathrm{prompt}, i}^{\mathrm{far}}} e^{-\mu_{\mathrm{late}, i}}} \\
& w_{\text {prompt }, i}^{\text {mid }}=\mathcal{P}_{i}(\text { prompt } ; \text { mid } \mid \text { hit }) \overline{\mathcal{P}}_{\text {prompt }, i}^{\text {near }} \\
& =\frac{1-e^{-\mu_{\mathrm{prompt}, i}^{\mathrm{mid}}}}{1-e^{-\mu_{\mathrm{prompt}, i}^{\mathrm{mid}}} e^{-\mu_{\mathrm{prompt}, i}^{\mathrm{far}}} e^{-\mu_{\mathrm{late}, i}}} \times\left(1-w_{\mathrm{prompt}, i}^{\mathrm{near}}\right) \\
& \left.w_{\text {prompt }, i}^{\text {far }}=\mathcal{P}_{i} \text { (prompt; far } \mid \text { hit }\right) \overline{\mathcal{P}}_{\text {prompt }, i}^{\text {near }} \overline{\mathcal{P}}_{\text {prompt }, i}^{\text {mid }} \\
& =\frac{1-e^{-\mu_{\mathrm{prompt}, i}^{\mathrm{far}}}}{1-e^{-\mu_{\mathrm{prompt}, i}^{\mathrm{far}}} e^{-\mu_{\mathrm{late}, i}}} \times\left(1-w_{\mathrm{prompt}, i}^{\mathrm{near}}-w_{\mathrm{prompt}, i}^{\mathrm{mid}}\right) \\
& w_{\text {late }, i}=\mathcal{P}_{i}(\text { late } \mid \text { hit }) \overline{\mathcal{P}}_{\text {prompt }, i}^{\text {near }} \overline{\mathcal{P}}_{\text {prompt }, i}^{\text {mid }} \overline{\mathcal{P}}_{\text {prompt }, i}^{\text {far }} \\
& =1-w_{\mathrm{prompt}, i}^{\mathrm{near}}-w_{\mathrm{prompt}, i}^{\mathrm{mid}}-w_{\mathrm{prompt}, i}^{\mathrm{far}}
\end{aligned}
$$

where they are time ordered such that the closest track has the best chance to trigger the discriminator. The late light distributions are simply averaged over,

$$
\hat{G}_{\text {late }, i}=\frac{1}{3}\left(G_{\text {late }, i}^{\text {near }}+G_{\text {late }, i}^{\mathrm{mid}}+G_{\text {late }, i}^{\mathrm{far}}\right)
$$

to preserve the normalization. Therefore, the time likelihood PDFs are,

$$
f_{t, i}=w_{\mathrm{prompt}, i}^{\mathrm{near}} G_{\mathrm{prompt}, i}^{\mathrm{near}}+w_{\mathrm{prompt}, i}^{\mathrm{mid}} G_{\mathrm{prompt}, i}^{\mathrm{mid}}+w_{\mathrm{prompt}, i}^{\mathrm{far}} G_{\mathrm{prompt}, i}^{\mathrm{far}}+w_{\text {late }, i} \hat{G}_{\text {late }, i}
$$

where the dependence on the corrected time, predicted charges, and track energies is implicit.

\subsubsection{Seeding the Fitter}

Now that an appropriate likelihood function has been constructed, initial parameters for the fitting routine must be found. There is an inherent instability trying to run the fitter from a random set of $\mathbf{u}$, or even the same starting $\mathbf{u}$ for all events. The reason, is that in the 15 dimensional parameter space, it is fairly easy for a parameter to get stuck in a local maximum. The goal, then is to find a "close" starting point for 
each parameter in the fit. Once we have a set of parameters to seed the full likelihood function Eqn. 4.2, the Minuit [79] fitting program is used to maximize the likelihood.

Rather than try to find all 15 parameters to use as a seed, the task will be to find each each track in succession while improving the parameters of the previous tracks at each step. The process starts with a guess for the first track based on the previous one-track fits that were performed. The next step is to freeze that track in the likelihood function while scanning for a second track in solid angle. Once a guess for the second track is found, a two-track fit is performed attaining a better guess at the parameters for both of those tracks. Those two tracks are then fixed in the likelihood function and a scan for the third track is performed. After finding that track, all three tracks are allowed to float in a three-track fit. At this stage, no guess is made as to the particle type of each track. A final phase of the fit is performed with various particle hypotheses and compared with the generic three-track fit. At this stage, a fit is chosen as the best guess for the tracks' types hypotheses and that fit is used for the particles' kinematics.

\subsubsection{One-Track Muon Fit}

To find the first track in an event some criterion is needed to determine if the muon-CCQE fit found one of the tracks, and tell how well it found a track. The first thing to check is how close the one-track fit vertex is to the true event vertex in the MC. Fig. 4.3 shows that the one-track fit is usually over a meter away from the true vertex. Having a vertex this far off will severely bias the direction of the track returned by the fit.

The question to ask at this point is not if the one-track fit found a track, but if it found a particle's Čerenkov ring? The difference is subtle in that the first requires that the event vertex was found, the second just requires that the fit found a spot on the wall close to where the real track was pointing. The reason for this is the detector is very good at finding rings on the wall but not necessarily so good at guessing where 


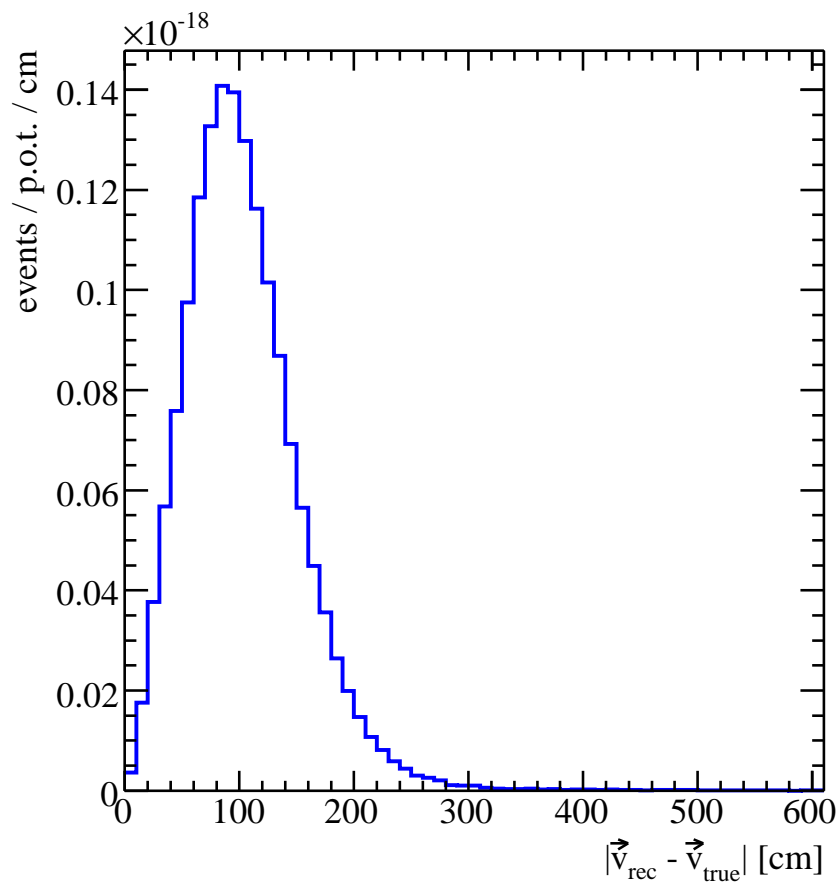

Figure 4.3: The distance between the one-track muon fit vertex and the true $\mathrm{CC} \pi^{0}$ vertex. Plotted are the number of events per proton on target (p.o.t.) per $\mathrm{cm}$ for signal events.

in the tank a track came from. To facilitate this inquiry, the track parameters $\mathbf{u}$ from the one-track fit need to be transformed into a vector that points from the center of the tank to the same spot that the reconstructed vector pointed to on the front face of the PMTs. That vector has a constant magnitude and is found by the transformation,

$$
\vec{r}=\hat{u} \times(\vec{v} \times \hat{u})+\hat{u} \sqrt{(\vec{v} \cdot \hat{u})^{2}+r^{2}-v^{2}}
$$

where $\hat{u}$ is the reconstructed direction unit vector, $\vec{v}$ is the reconstructed event vertex, and $r=552.9 \mathrm{~cm}$ is the distance from the center of the tank to the front face of the PMTs. Also, $r$ is the magnitude of $\vec{r}$ by construction. To compare this vector to the true event information it is simply shifted by the true event vertex,

$$
\vec{r}_{\text {shift }}=\vec{r}-\vec{v}_{\text {true }}
$$

which keeps its spot-on-the-wall fixed. To compare this with a true track, the criterion 
angle is formed by simply taking the dot product of the shifted unit vector with a true track's direction,

$$
\cos \xi=\hat{u}_{\text {true }} \cdot \hat{r}_{\text {shift }}
$$

where the angle $\xi$ is the criterion that decides if the reconstructed track found a true track. Fig. 4.4 shows how well the muon one-track fit found the true muon in $\mathrm{CC} \pi^{0} \mathrm{MC}$

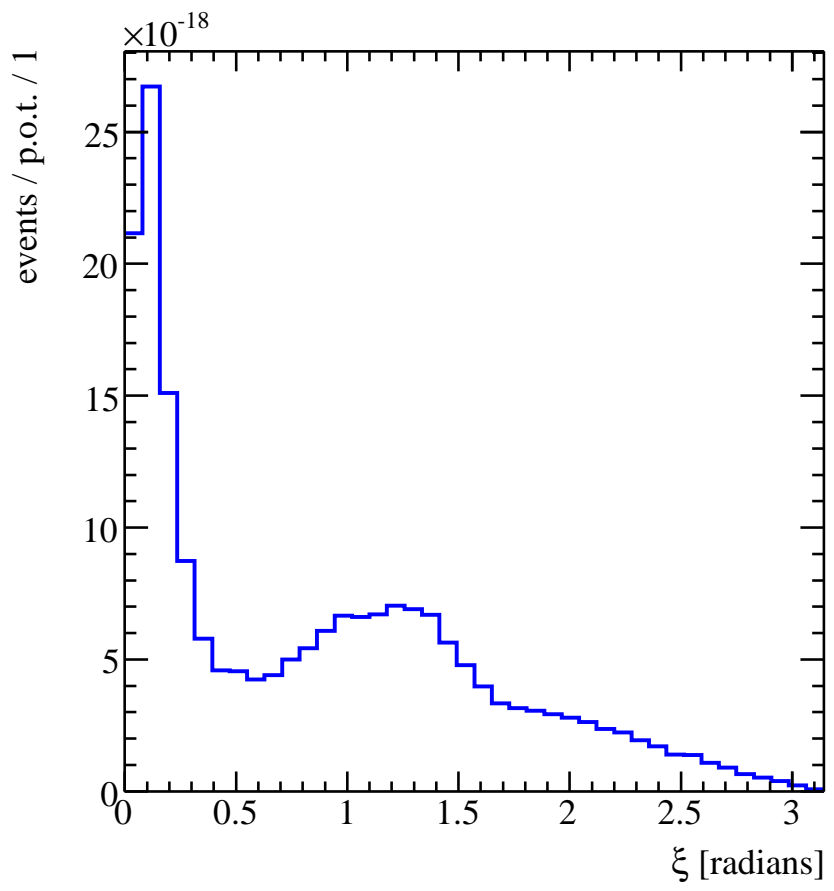

Figure 4.4: The "angle" between the one-track muon fit and the true muon track in a $\mathrm{CC} \pi^{0}$ event.

events. The sharp peak below 0.5 radians shows that about $30 \%$ of the time the onetrack found the muon in the event. Beyond 0.5 radians lie the bulk of the events. Clearly, the one-track fit is as likely to find another track as it is to find the muon. Making this angle versus all three tracks in the event, then taking the one with the smallest angle shows if the fit found a ring in the event. Fig. 4.5 shows the angle between the one-track fit and the closest track in the event for the same events in Fig. 4.4. Almost all of the events are in the peak below 0.5 radians. This implies that even though the track did 


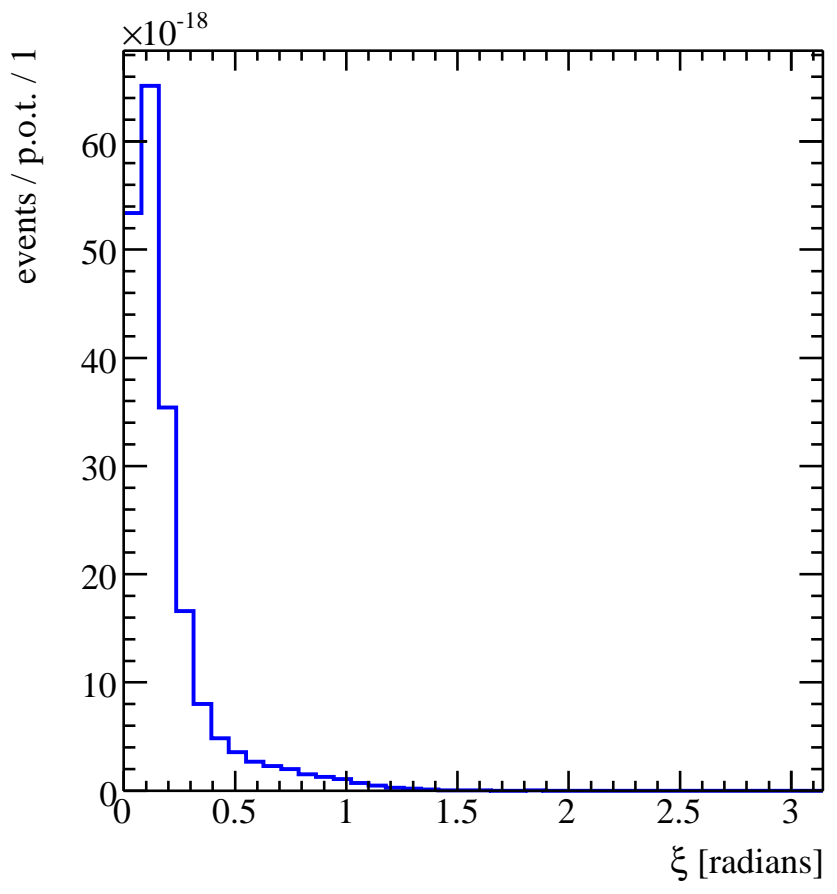

Figure 4.5: The smallest "angle" between the one-track muon fit and any track in a $\mathrm{CC} \pi^{0}$ event.

not find the muon in the event, it did find one of the Čerenkov rings.

As a starting point, it would seem that the one-track fit will provide an adequate seed for the first track. However, knowledge of the track type is still obfuscated. For now, the vertex will be seeded by the flawed one-track vertex and will be improved through the course of the staged seeding procedure.

\subsubsection{Solid Angle Scans}

To find additional tracks in the event a scan in solid angle is performed. The scan is performed around the current event vertex, with all previously "found" tracks frozen in the likelihood function. After each scan, a fit is performed allowing the previous tracks and the newly found track to float. The purpose of this is two-fold; to find a better event vertex, and to correct the track energies. Each scan is performed using a 
grid of 400 points, chosen to be fine enough to cover the whole space adequately, and coarse enough so that the 1280 PMTs can easily choose between grid points.

The problem of distributing an arbitrary number of points, $N$, on a sphere is an unsolved problem in mathematics with applications to electrostatics [80]. The solution usually comes from attempting to place equal charges on a perfectly conducting sphere. The minimum energy solution will equally distribute the charges. However, there is no closed form solution for an arbitrary number of charges. An approximation for large $N$ is found by placing points at both poles and then following a spiral pattern, distributing the remaining $N-2$ points. The algorithm is given by [80]:

$$
\begin{array}{cll}
h_{k}=-1+2 \frac{(k-1)}{(N-1)} & , \quad 1 \leq k \leq N \\
\theta_{k}=\quad \cos ^{-1}\left(h_{k}\right) & , \quad 1 \leq k \leq N \\
\phi_{k}=\left(\phi_{k-1}+\frac{3.6}{\sqrt{N} \sqrt{1-h_{k}^{2}}}\right) \% 2 \pi & , \quad 2 \leq k \leq N-1 \\
\phi_{1}=\phi_{N}=0 &
\end{array}
$$

where $\theta$ and $\phi$ are taken as the beam coordinates ${ }^{5}$. Fig. 4.6 shows the 400 point uniform grid projected onto a 2-dimensional surface. The red dots show the locations of the detector coordinates in the projection. As the scan takes place around the current event vertex, the coordinate directions (invariant with respect to position) do not point to the same locations on the tank wall for any given scan.

The first scan fixes the one-track muon fit as the first particle. A second muon track, with the same vertex and $200 \mathrm{MeV}$ of kinetic energy, is scanned about the grid with the negative logarithm of the likelihood recorded at each scan point. This surface will be referred to as the "likelihood surface" for brevity. Fig. 4.7 shows the likelihood surface produced by the first scan for a "cherry-picked" event. The wall positions of the true tracks relative to the reconstructed vertex are the large red (muon) and brown (photon) dots. The red " $\mathrm{x}$ " is the one-track fit direction which is fixed in this scan. The

\footnotetext{
${ }^{5} \hat{z}=(0,0), \hat{x}=(\pi / 2,0)$, and $\hat{y}=(\pi / 2, \pi / 2)$
} 


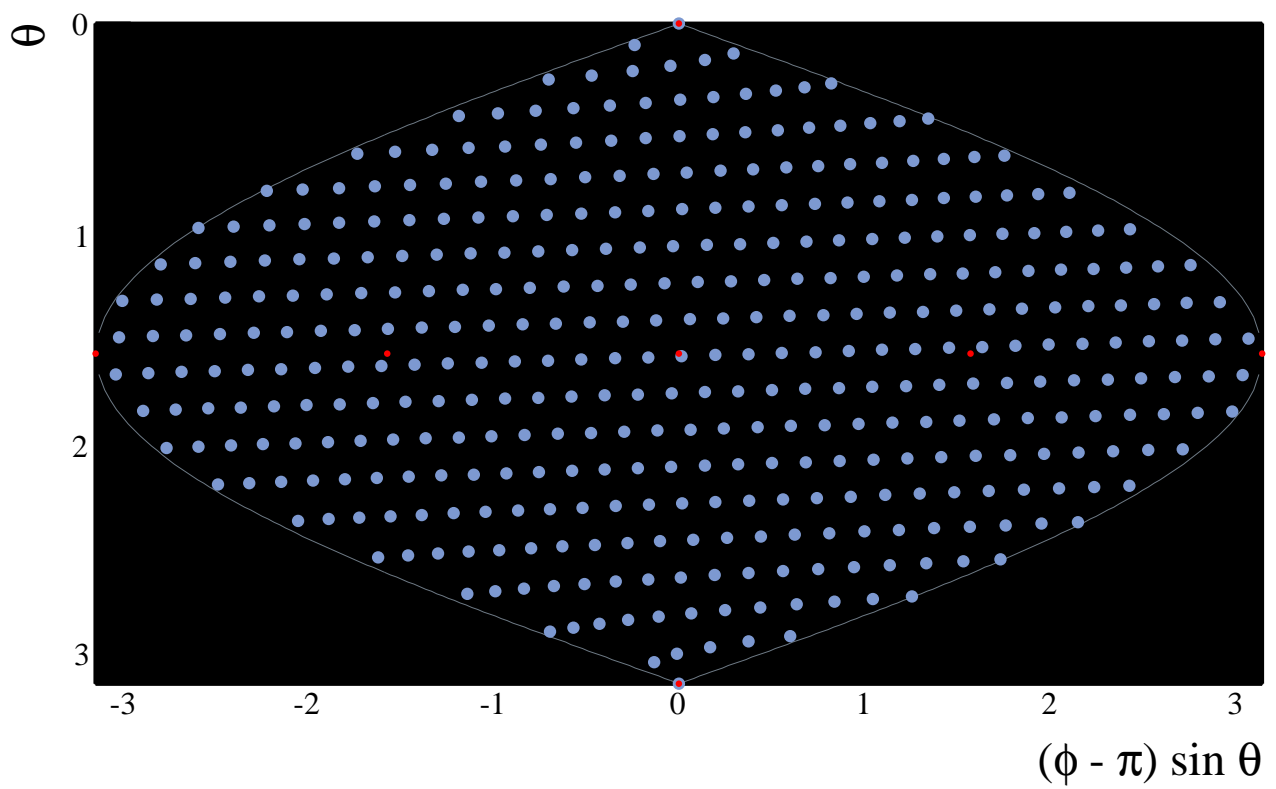

Figure 4.6: A roughly evenly spaced grid of 400 points on a sphere projected onto a flat surface, the left and right edges fold back onto each other. The coordinates, $\theta$ and $\phi$, are relative to the detector coordinates. The red dots are the angular directions of the axes: $+\hat{z}$ (beam) is the top point of the grid $(0,0)$, and $-\hat{z}$ is at the bottom $(0, \pi) ;+\hat{x}$ (beam left) is at both $(-\pi, \pi / 2)$ and $(\pi, \pi / 2) ;-\hat{x}$ (beam right) is the dead center of the plot, $(0, \pi / 2) ;+\hat{y}$ (vertical) is at $(-\pi / 2, \pi / 2)$ and the $-\hat{y}$ (downward) is at $(\pi / 2, \pi / 2)$.

yellow dot is the minimum of the likelihood surface. The first thing to notice is that the muon fit found one of the photons instead of the true muon. The second notable feature is that there are local minima in the likelihood surface at the remaining photon's and muon's positions. This scan found the second photon.

To find the third track a second solid scan is performed. Prior to that, a fit is performed allowing the two tracks that have already been found to float. The reason for this is to fill in the minimum that was found by the second track, and deepen the minimum of the third track. Part of the reason that a staged approach works is because sometime the locations of a particle is dominated by a deep minimum due to another. This effectively causes a flattening to the wall of the minimum, but might not create its own local minimum. Re-fitting the previously found track allows for the filling in of their 


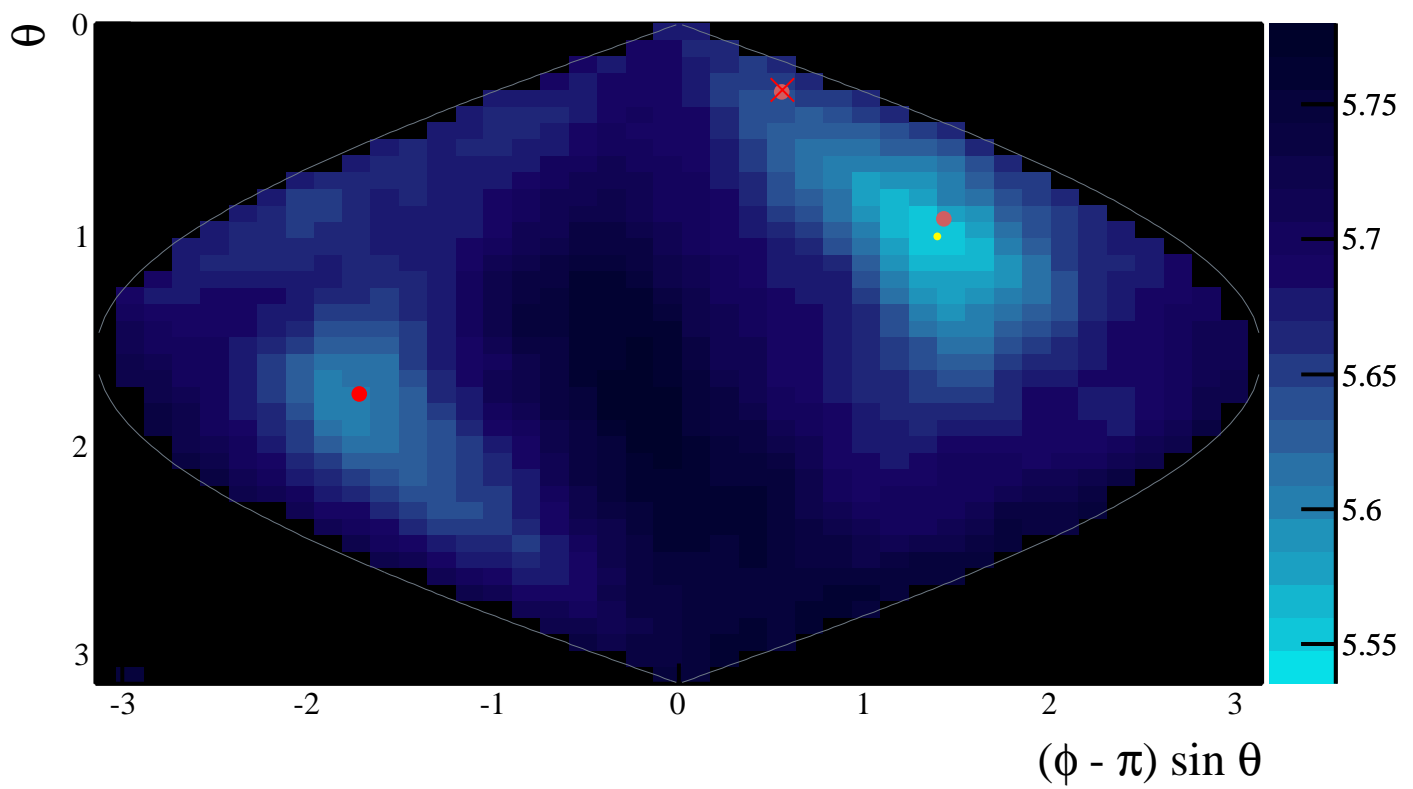

Figure 4.7: A likelihood scan in solid angle of a true $\mathrm{CC} \pi^{0}$ event. The red dot is the true muon direction relative to the one-track muon vertex. The brown dots are the directions of the true photons. The red cross is the result of the one-track muon fit. Finally, the yellow dot shows the location of the minimum of the likelihood surface. The one-track fit clearly found a photon; the likelihood scan found the other photon.

minima and a magnification, or even creation of the next most prominent minimum.

The results of the two-track muon fit and scan for the third track are shown in Fig. 4.8 for the same event. The scan was also performed with a $200 \mathrm{MeV}$ muon track. In this fit, the vertex has improved because each additional ring found in the event adds a strong constraint on where the event began. If the one-track fit found a photon in the event, there is a good chance that the event vertex it found is at the start of the electromagnetic shower, where the photon converted, opposed to the start of the muon track. Adding an additional ring, even if it is another photon, forces the vertex towards the correct location. In Fig. 4.8, the effect of floating each track has caused the minimum found by the first scan to fill in. What was a local minimum in Fig. 4.7 has now become the global minimum and the correct location of the muon in the event. 


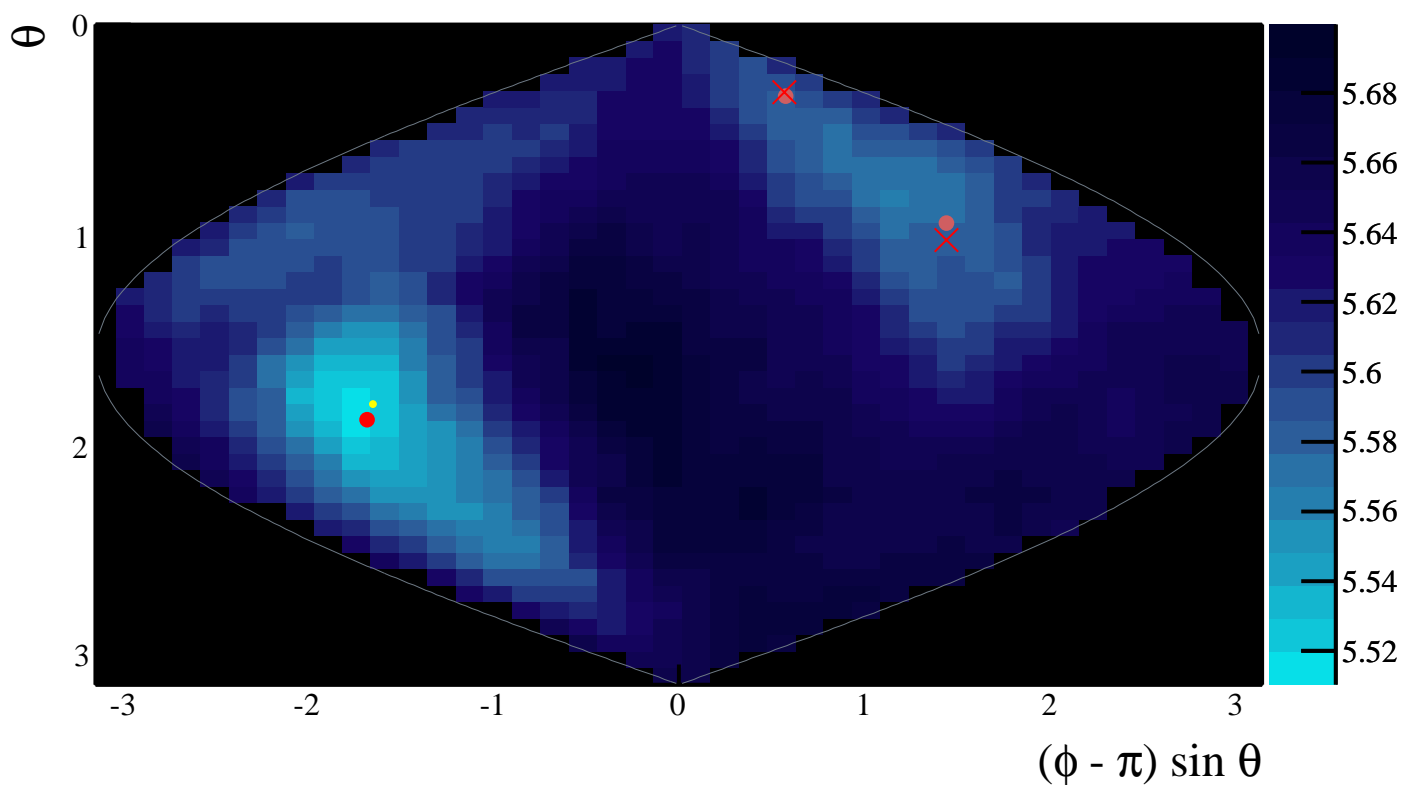

Figure 4.8: After a two-track muon fit seeded by the results of the first scan, a second scan is performed. Comparing with Fig. 4.7 the surface shows a dimming of the photon peak and a brightening of the muon peak. The scan clearly finds the muon. The red crosses are the results of the two-track fit.

After the second scan, all three tracks are allowed to float in a three-track muon fit. Fig. 4.9 shows the result of that fit. Adding the third track has again helped with the vertex position. At this point, the tracks were found in a generic manner, with muons acting as a generic particle. This three-track generic fit will act as the basis for determining the track types and eventually the fit kinematics.

\subsubsection{Final Fits}

The three-track generic fit provides a geometric basis for the event. The remaining steps involve finding the muon, setting the photon conversion lengths, and getting the tracks' kinetic energies. As there are only three possible configurations that a muon and two photons can have, three simultaneous fits are performed where two of the track types are substituted for photon hypotheses. The first stage of these fits fixes all 


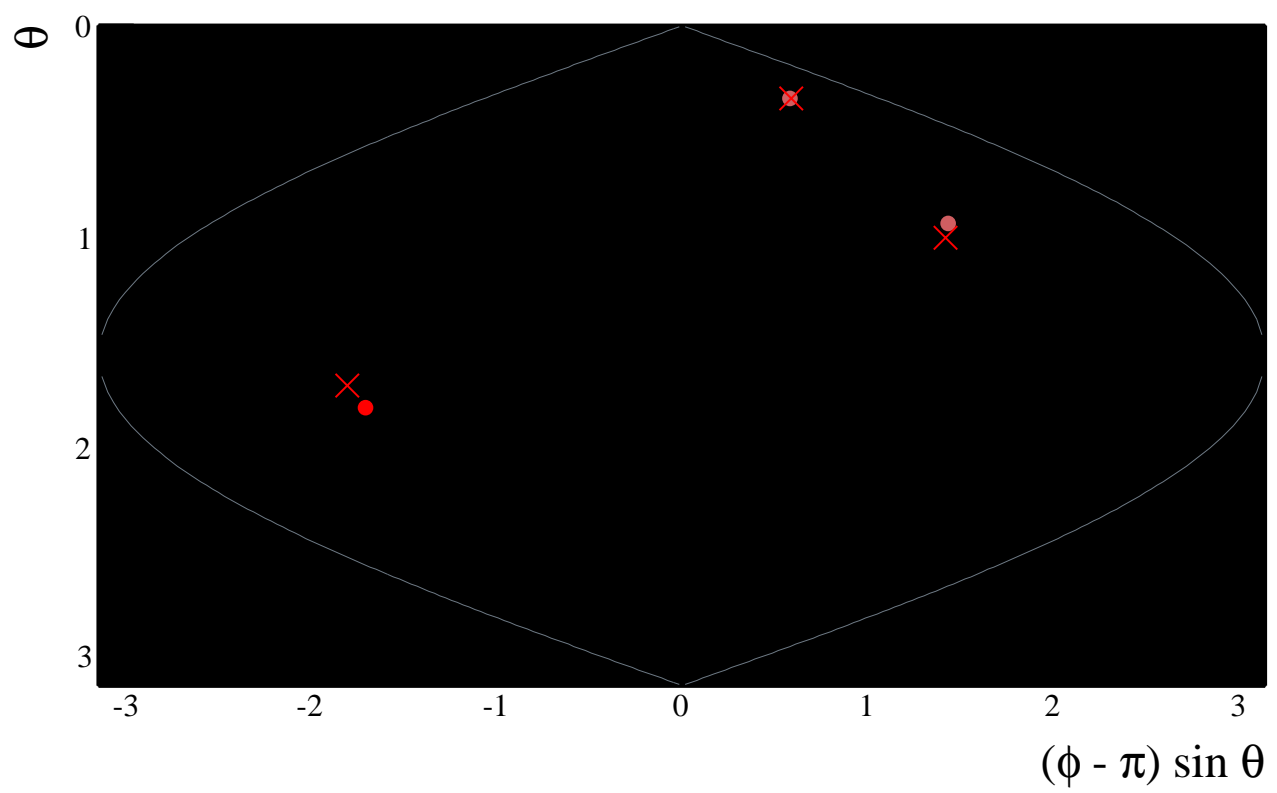

Figure 4.9: A three-track muon fit is performed with the seed set by the second scan. This fit improves the event vertex and track directions.

the parameters except the track energies, and the conversion lengths. The conversion lengths for the photons are seeded with $50 \mathrm{~cm}$, which is roughly the conversion length of photons in the mineral oil. The goal of this fit is to get a better track hypothesis and conversion length guesses to seed the final fit. After this fit, a fully unconstrained 15 parameter fit is performed. The output of this fit is then reseeded using Minuit's Improve function [79]. This refit is chosen if its likelihood is smaller than the previous fit. The purpose of the Improve fit is to check if the regular fit left any of the parameters stuck in a local minimum.

\subsubsection{Particle Identification}

To choose the fit that correctly identifies the track type configuration the fit likelihood is combined with the muon decay information. An electron from a stopped muon decay is called a Michel electron. For each fit, a term is added to the fit likelihood 
that compares the direction to the Michel electron vertex from the fit vertex and the direction of the muon track. A Gaussian form for the likelihood is assumed,

$$
\mathcal{L}_{\text {michel }}=\exp \left(-\frac{\lambda\left(\hat{u}_{\mu}-\hat{u}_{\text {michel }}\right)^{2}}{8 \sigma^{2}}\right)=\exp \left(-\frac{\lambda \sin ^{2}(\theta / 2)}{2 \sigma^{2}}\right)
$$

where $\hat{u}_{\mu}$ is the muon direction, $\hat{u}_{\text {michel }}$ is the direction to the Michel vertex, $\theta$ is the angle between the muon and the Michel direction, $\sigma$ is the distance dependent spread of the angular separation between the muon and Michel directions, and $\lambda$ is a correction tuned to maximize track identification (described below). This likelihood can be simply added to the fit likelihood in their negative logarithms as,

$$
\chi^{\prime 2}=-\log \mathcal{L}_{f i t}+\frac{\lambda \sin ^{2}(\theta / 2)}{2 \sigma^{2}} .
$$

where $\mathcal{L}_{f i t}$ is one of the three fit configuration likelihoods. The minimum is chosen between the 3 fit configurations for the final set of fit kinematics.

The parameter $\sigma$ is found by fitting $\sin (\theta / 2)$ versus Michel distance in slices of distance (see Fig. 4.10). Each slice is fit to Landau distribution with the points set at two sigma more than the distribution's mean. This was chosen to get a better shape fit for the distribution, as the overall normalization can be absorbed into $\lambda$. Additionally, this reduces the dependence of $\sigma$ on the position of the Landau peak, as the first few slices have poorer peak resolution. Fig. 4.11 shows $\sin (\theta / 2)$ versus Michel distance. The function shows the fit to the slice points and defines $\sigma$ for all distances less than 500 $\mathrm{cm}$. Above $500 \mathrm{~cm}$ the function is fixed at $\sigma(500 \mathrm{~cm})$. In practice, the reconstructed muon angle is used while the distributions were fit using the true muon direction.

The free parameter $\lambda$, is found by scanning over a range and choosing the value that maximizes the muon yield (fraction of time where the muon fit finds the muon). Fig. 4.12 shows the muon yield as a function of $\lambda$. The value of $\lambda=0.02$ is found to maximize the muon yield. This likelihood method of particle identification has more than doubled the muon yield compared to the one-track muon fit. Additional cuts on the sample will eventually improve the yield. 

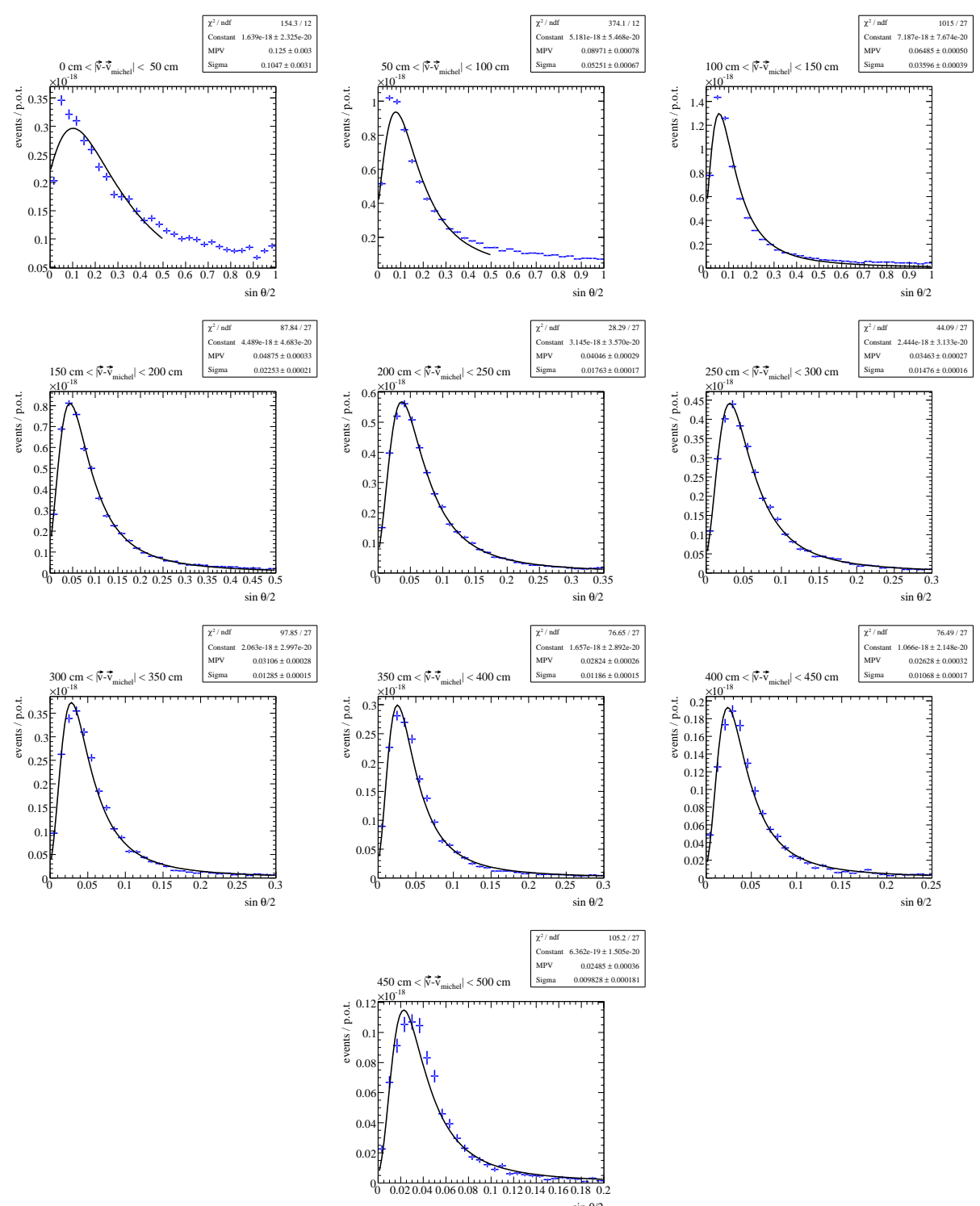

Figure 4.10: The angle between the true muon direction and the Michel direction in slices of $50 \mathrm{~cm}$ over a range of $500 \mathrm{~cm}$. Plotted are the number of events per proton on target (p.o.t.). Each plot has a different range of angle in an attempt to plot the bulk of the distributions.

Returning to the sample event, Fig. 4.13 shows the fit chosen by Eqn. 4.34. For this event, the configuration was correctly identified. However, one particular "cherrypicked" event does not prove that the fitter will work. The results of this section will show that particle identification has improved from roughly $30 \%$ to $67 \%$ for all $\mathrm{CC} \pi^{0}$ 


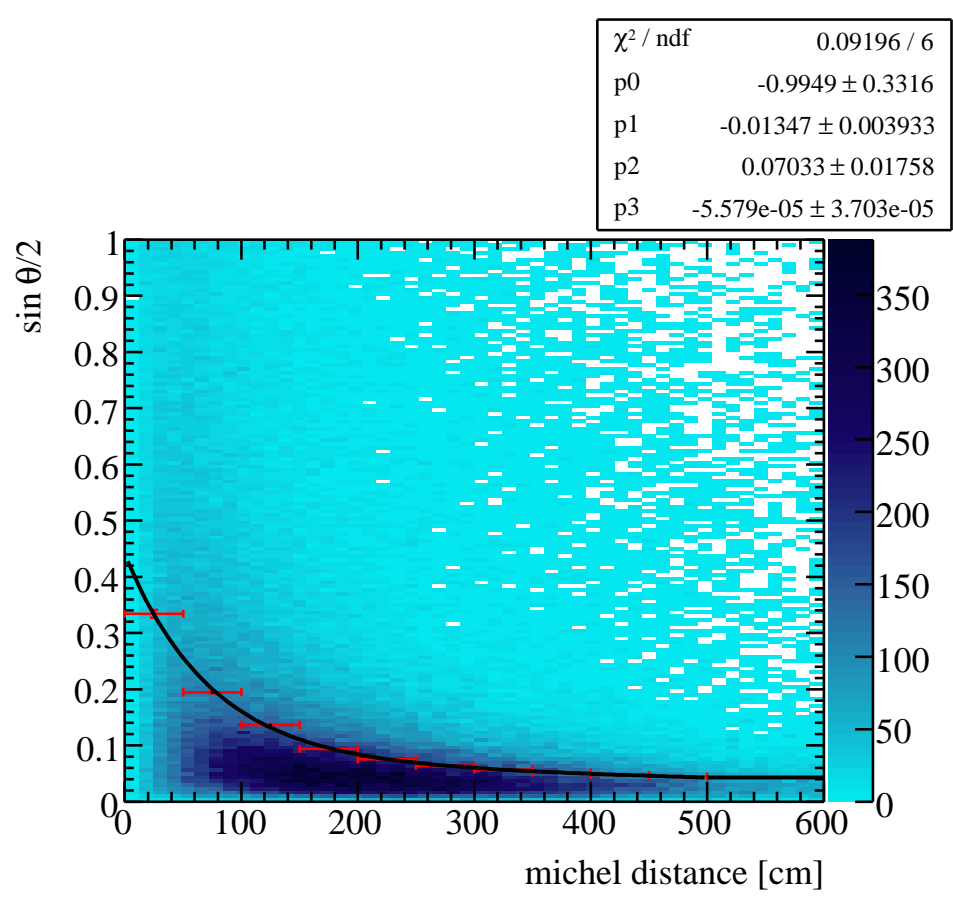

Figure 4.11: The true muon with respect to Michel angle versus the Michel distance. The curve is fit to twice the sigma above the mean gathered from the fits in Fig. 4.10. This choice was arbitrary, and was designed to penalize the Michel portion of the likelihood at small Michel distances.

events. To see more directly, Fig. 4.14 shows the muon true direction versus fit direction angle, $\xi$, for all $\mathrm{CC} \pi^{0}$ for both the muon-CCQE fit and this final $\mathrm{CC} \pi^{0}$ fit. The muon identification has not only greatly improved, but also reduced the number of mis-IDs. Most of the mis-IDed portion of the $\mathrm{CC} \pi^{0}$ fit curve is from events that hit a fundamental limitation of what the MiniBooNE detector can reconstruct.

\subsection{Fit Results}

The fitter is checked by looking at how well it reconstructs true $\mathrm{CC} \pi^{0}$ events. The claim at the start was that the muon-CCQE fit provided a poor event vertex. The first thing to check is how well the three-track fit improved the event vertex. Fig. 4.15 shows the improvement over the one-track muon fit. The vast bulk of the events are 


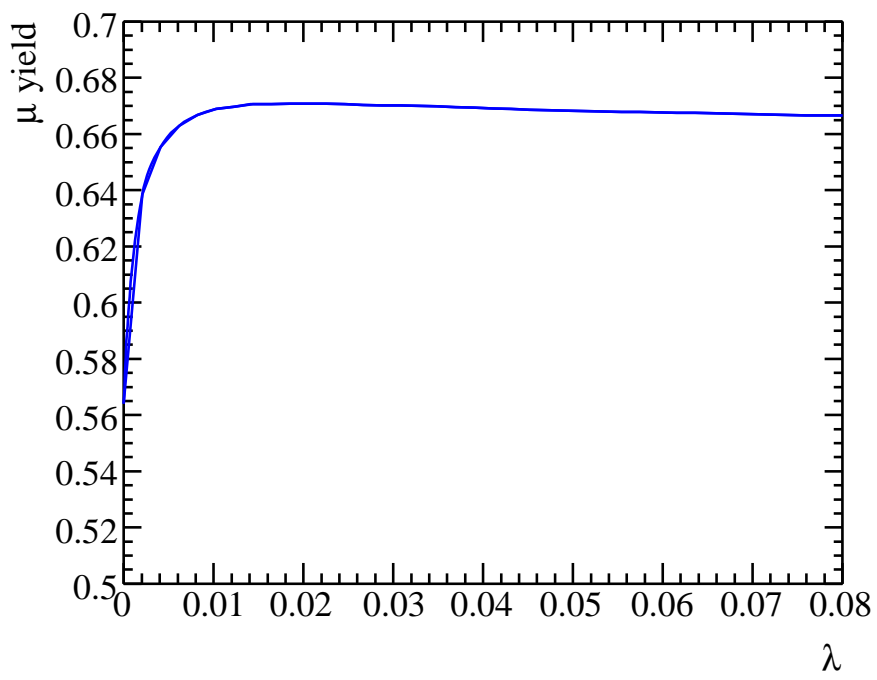

Figure 4.12: The fraction of events where the likelihood (Eqn. 4.34) chose the correct particle type configuration versus $\lambda$. The maximum of this curve is at $\lambda=0.02$.

now less than $50 \mathrm{~cm}$ from the true vertex where the one-track vertex tended to be over $100 \mathrm{~cm}$ away.

The reconstructed muon kinematics are compared in Fig. 4.16. The reconstruction shows a distortion in the kinetic energy spectrum that is a consequence of mis-ID. The mis-ID fraction is $33 \%$. The overall energy resolution is $8 \%$ in the peak. The residual shows a mis-IDed "swoosh" effect at low energies. The angular distribution is slightly more forward peaked than the MC.

The reconstructed photons are sorted by how well they found a true photon. The decision is based on taking the minimum $\chi^{2}$ between each true photon and the shifted reconstructed photon directions. The best found photon reconstruction is shown in Fig. 4.17. The energy resolution is $19 \%$ over the whole energy range. The angular agreement is excellent by definition. The conversion length shows a pile-up at zero 


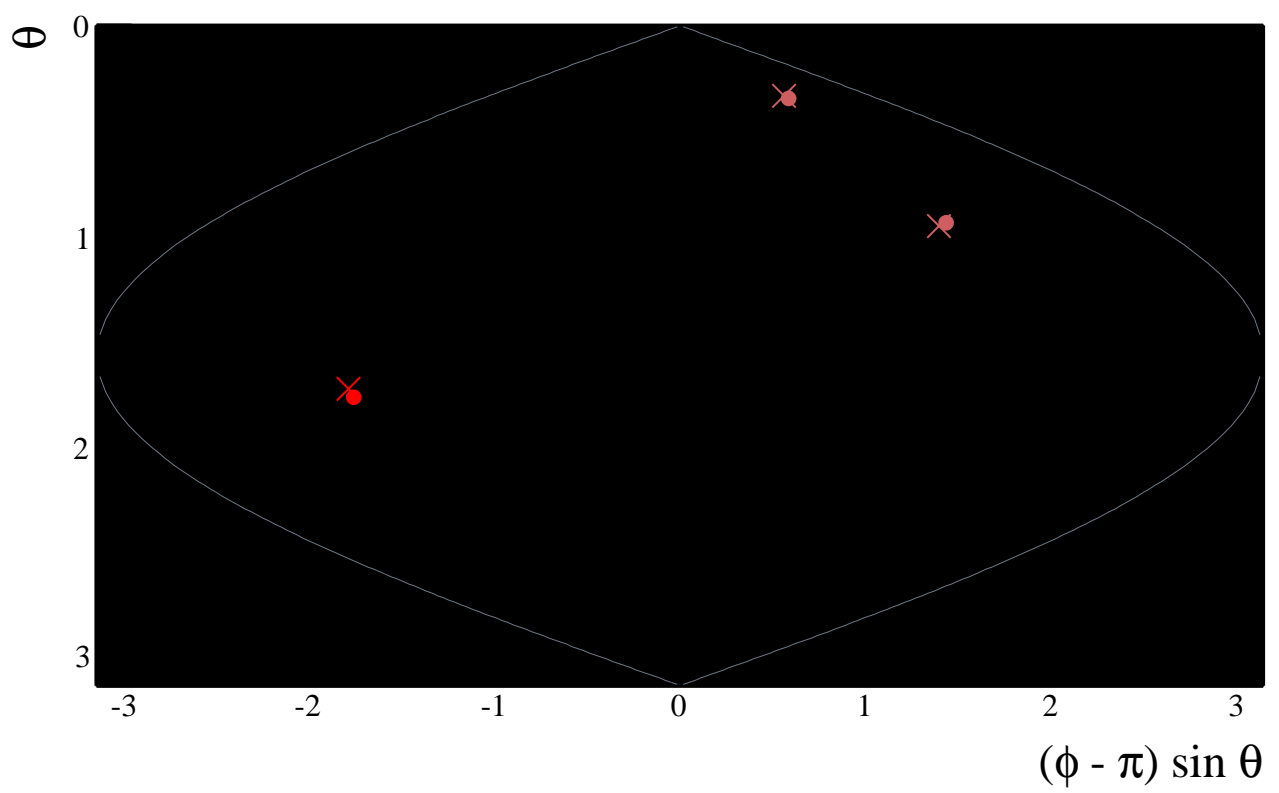

Figure 4.13: The final three-track fit with two of the muons swapped out for photons during the fit. The reconstructed photons are brown and match up with the true photons.

length which is mainly due to mis-IDs.

By definition the second photon is worse. Fig. 4.18 shows the second photon's reconstructed kinematics. The energy resolution is significantly worse at 28\%. Also, the entire effect of mis-IDed events are absorbed into this photon. Interestingly enough, the conversion length comes out the same as the better reconstructed photon.

The photon reconstruction does not demonstrate how well the events were reconstructed, partly because of the ambiguity in assigning true to reconstructed photons. The reconstructed $\pi^{0}$ does not suffer from this ambiguity as it is a combination of both photons' four-vectors. Fig. 4.19 shows the reconstructed pion kinematics. The plots show that the momentum resolution is $14 \%$, on par with the reconstructed muon's energy resolution. The reconstructed angle also matches the true pion angle fairly well but is slightly more forward peaking than the true distribution. With the pion, the mass can also be reconstructed, and after removing the very obvious mis-IDed events, 


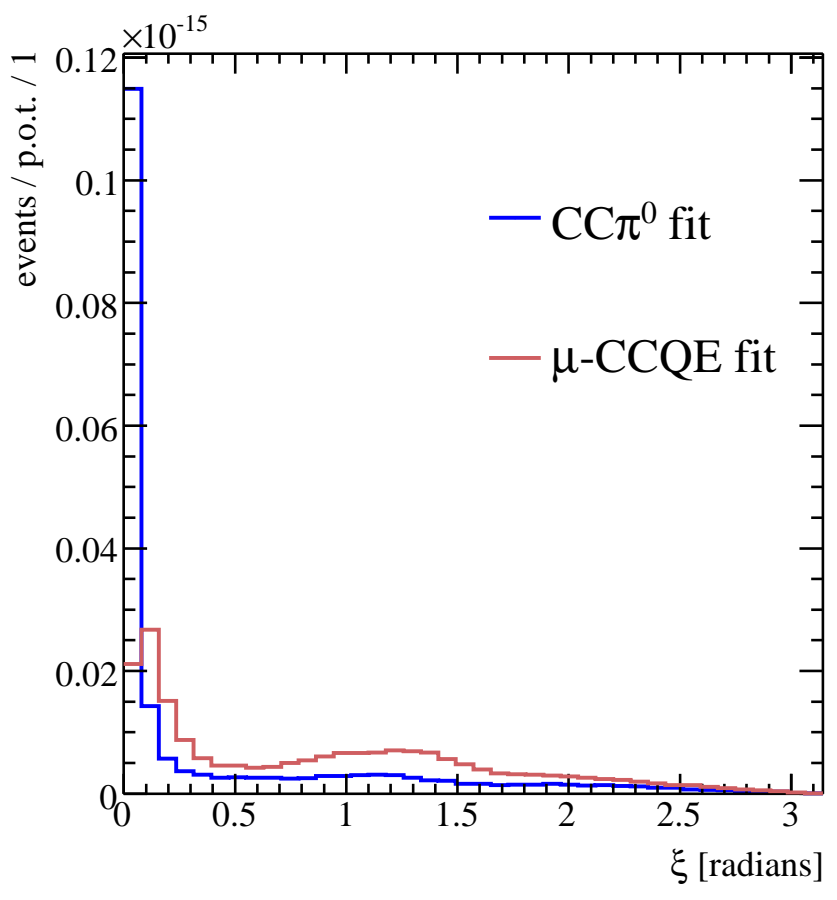

Figure 4.14: The angle between the fit muon and the true muon for both the $\mathrm{CC} \pi^{0}$ fitter and the muon-CCQE fitter (same curve as Fig. 4.4). A dramatic improvement over the muon-CCQE is apparent for both the muon yield and the muon angular reconstruction.

matches the true $\pi^{0}$ peak to within a few $\mathrm{MeV} / c^{2}$.

The take-away message from this section is that the fitter works most of the time, though occasionally has drastic misreconstructions. The first goal of the analysis will be to decide on an acceptable level of misreconstruction, and use the reconstructed quantities to reject as much misreconstructed and mis-IDed events as possible while not biasing the final physics. The rejection of misreconstructed events will be addressed in $\S 4.5$.

\subsection{Neutrino Interaction Kinematics}

The $\mathrm{CC} \pi^{0}$ fitter is all for naught if it is unable to infer information about the incident neutrino. Fig. 4.20 shows a tree level Feynman diagram for a $\mathrm{CC} \pi^{0}$ event 


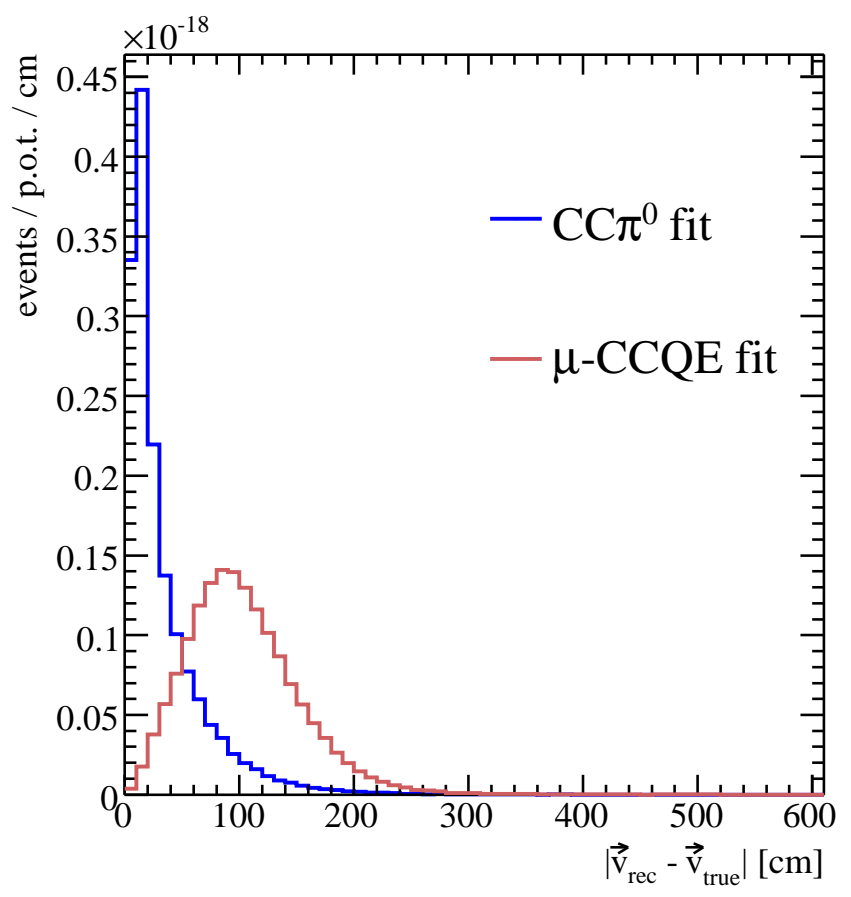

Figure 4.15: The fit vertex versus true event vertex for both the $\mathrm{CC} \pi^{0}$ fitter and the muon-CCQE fitter. The fit shows a rather dramatic improvement where the bulk of the event vertices are within $50 \mathrm{~cm}$ of their true vertex.

propagating through a delta resonance. The initial and final states $\left(\nu_{\mu} n \rightarrow \mu^{-} \pi^{0} p\right)$ must conserve 4-momentum, regardless of the happenings in the internal states. Explicitly,

$$
p_{\nu}+p_{n}=p_{\mu}+p_{\pi}+p_{p}
$$

where $p_{i}$ are the 4-momentum of particle $i$, and that $p_{\pi}=p_{\gamma 1}+p_{\gamma 2}$. Defining $p_{X} \equiv$ $p_{\mu}+p_{\pi}$, rearranging and squaring, the expression becomes,

$$
\left(p_{\nu}+p_{n}-p_{X}\right)^{2}=m_{p}^{2}
$$

where the proton mass has been substituted in. Clearly, in this form, knowledge of the proton's momentum is unnecessary. Since $p_{X}$ is fully reconstructed, this leaves one equation and six unknown quantities. At least five need to be constrained in some manner. The first assumption is that the neutrino is traveling in the beam direction. 

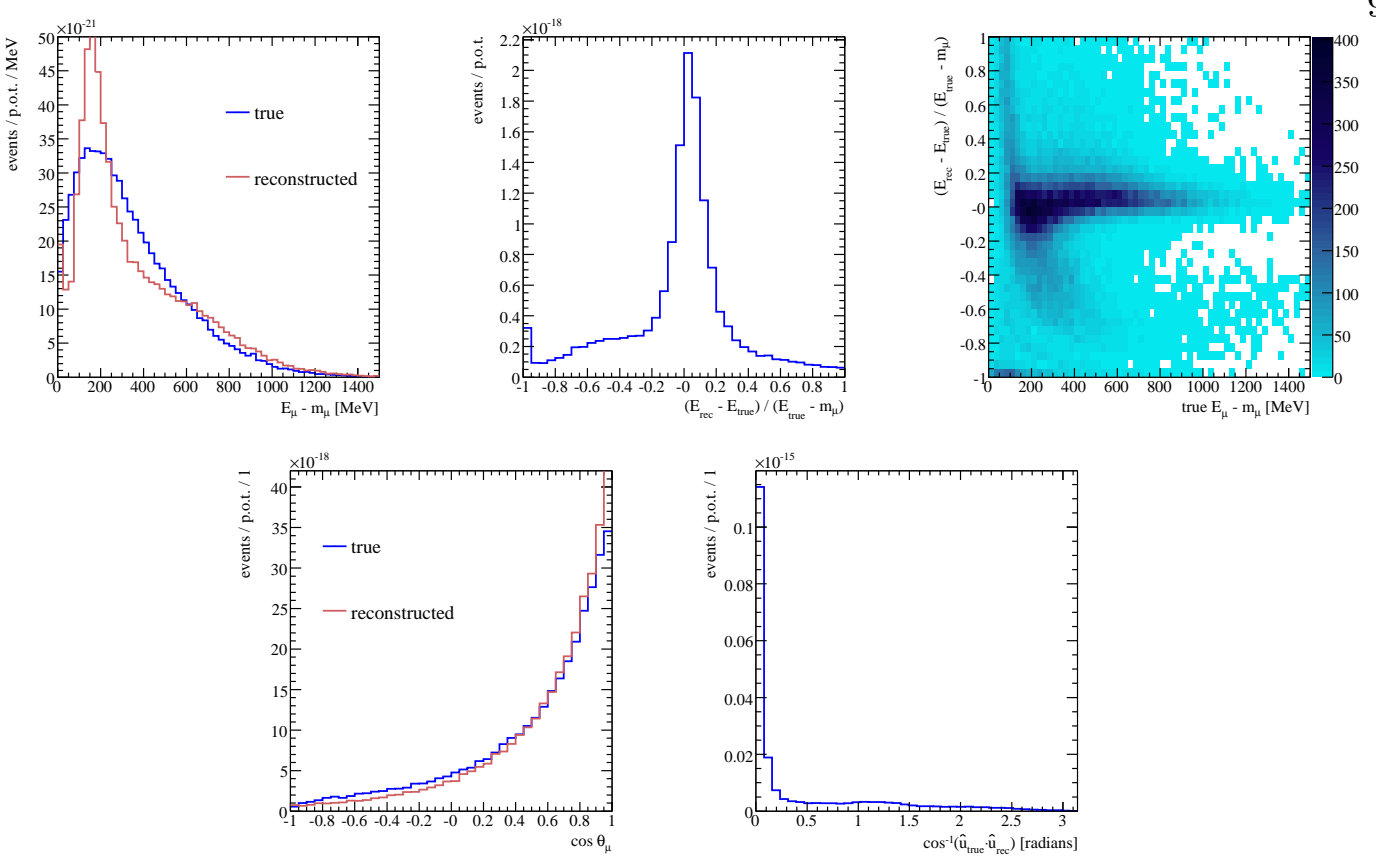

Figure 4.16: The muon fit kinematics along with the fit residuals. Top left: The kinetic energy for true and reconstructed events. Top center: The energy residual with an $8 \%$ energy resolution in the peak. Top right: The residual vs true kinetic energy. Bottom left: The angle wrt the beam direction for true and reconstructed events. Bottom right: The angle between the true and reconstructed directions.

The MiniBooNE detector is positioned $450 \mathrm{~m}$ from the beam dump, far enough away to make any possible angle that the neutrino could have negligible.

The second assumption is that the neutron is at rest in the nucleus. This is a poor assumption because the neutron can have around $\sim 1 \mathrm{GeV} / c$ of Fermi momentum in the nucleus. However, as long as the reconstructed observable is representative of the true quantity, the unfolding technique described in $\S 5.3 .5$ will correct for the effects of this assumption.

Evaluating Eqn. 4.37 in the laboratory frame, assuming a neutrino traveling in the beam direction, a stationary neutron, and no neutron binding energy, the neutrino 

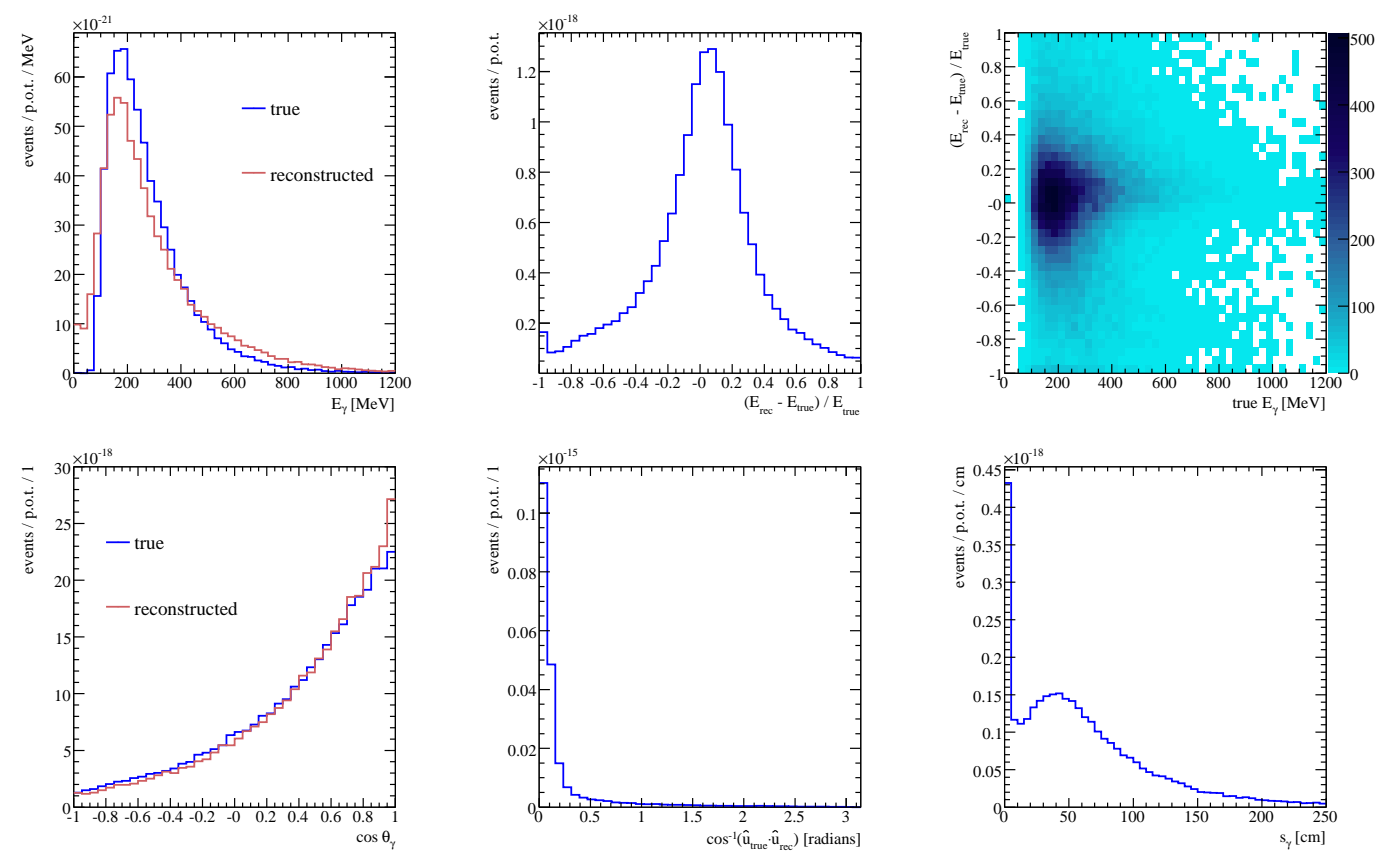

Figure 4.17: The first fit photon's kinematics along with the fit residuals. Top left: The energy for true and reconstructed events. Top center: The energy residual with an 19\% energy resolution in the peak. Top right: The residual vs true kinetic energy. Bottom left: The angle wrt the beam direction for true and reconstructed events. Bottom center: The angle between the true and reconstructed directions. Bottom right: The reconstructed conversion length.

energy $^{6}$ is,

$$
E_{\nu}^{\mathrm{CC} \pi^{0}}=\frac{m_{p}^{2}-m_{n}^{2}-m_{X}^{2}+2 E_{X} m_{n}}{2\left(m_{n}-E_{X}+\left|\mathbf{p}_{X}\right| \cos \theta_{\nu X}\right)}
$$

where $p_{X} \equiv p_{\mu}+p_{\gamma 1}+p_{\gamma 2}$, the 4-momenta returned by the $\mathrm{CC} \pi^{0}$ fitter, $\theta_{\nu X}$ is the angle between the neutrino and the composite, fictitious particle, $X$. Fig. 4.21 shows the neutrino energy calculated for $\mathrm{CC} \pi^{0}$ events using Eqn. 4.38. The reconstructed neutrino energy shows a linear distortion that gets worse at higher energy. However, the energy resolution for the bulk of the events is $11 \%$ and gets worse at high energy. Most of the distortion is due to misreconstruction and will be addressed through quality cuts and unfolding ( $§ 5.3 .5)$.

\footnotetext{
${ }^{6}$ To derive the CCQE neutrino energy formula replace $X \rightarrow \mu$; the resonant $\mathrm{CC} \pi^{+}$neutrino energy formula replace $X \rightarrow \mu$ and $n \rightarrow p$.
} 

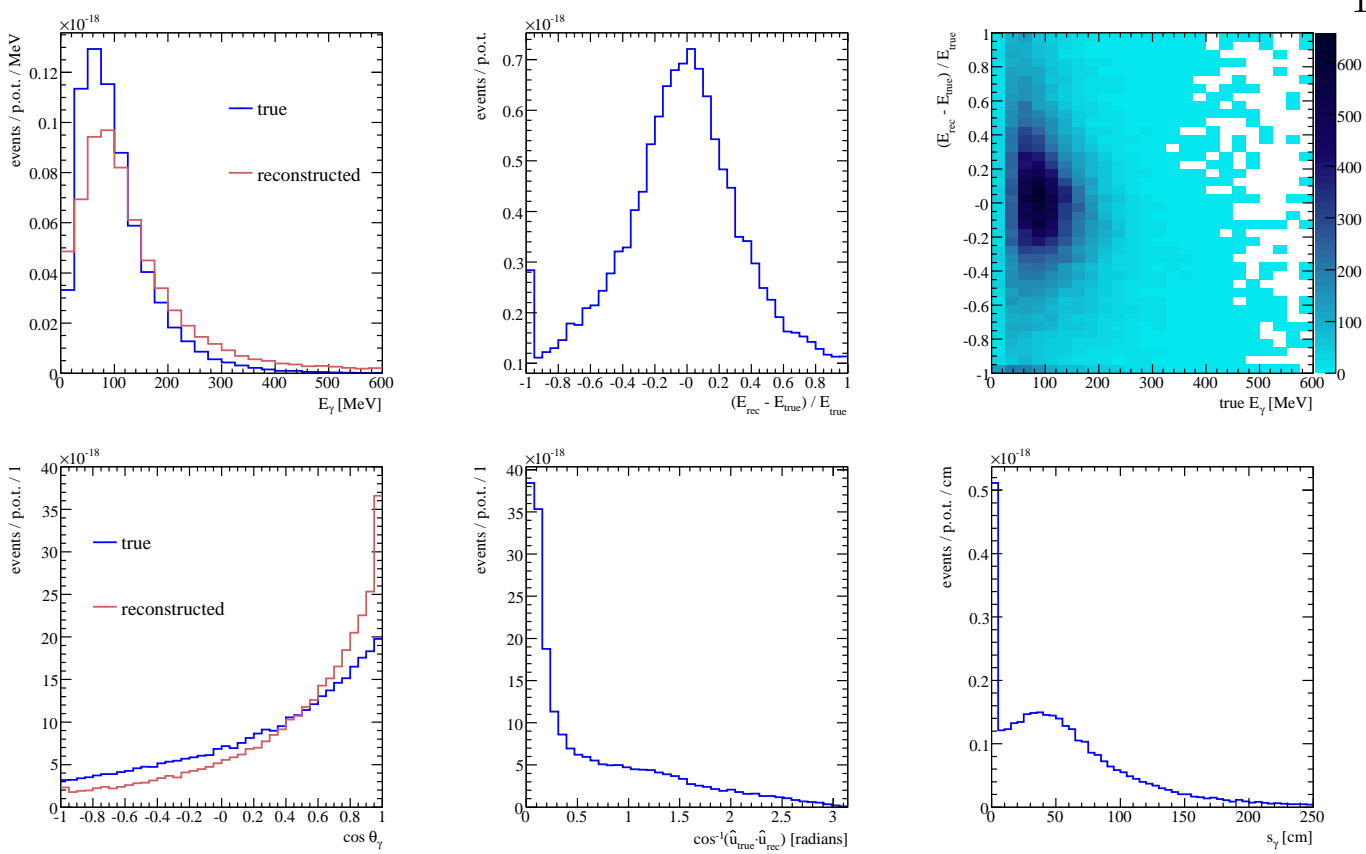

Figure 4.18: The second fit photon's kinematics along with the fit residuals. Top left: The energy for true and reconstructed events. Top center: The energy residual with an $28 \%$ energy resolution in the peak. Top right: The residual vs true kinetic energy. Bottom left: The angle wrt the beam direction for true and reconstructed events. Bottom center: The angle between the true and reconstructed directions. Bottom right: The reconstructed conversion length.

\subsection{1 $\quad \mathrm{Q}^{2}$}

Under the assumption that $\mathrm{CC} \pi^{0}$ events are dominated by the tree-level diagram (Fig. 4.20), the internal states can be reconstructed as all external states have been specified $^{7}$. The 4-momentum transfer to the hadronic system, $Q$, is often represented by its relativistic invariant, $Q^{2} \equiv-q^{2}$, where $q$ is the 4 -momentum of the $W^{ \pm}$boson. From Fig. 4.20 it is clear that the 4-momentum transfer can be constructed from just the lepton current in the diagram as,

$$
Q^{2}=-\left(p_{\nu}-p_{\mu}\right)^{2}=2 E_{\nu}\left(E_{\mu}-\left|\mathbf{p}_{\mu}\right| \cos \theta_{\nu \mu}\right)-m_{\mu}^{2}
$$

where $\theta_{\nu \mu}$ is the angle between the incident neutrino and the outgoing muon.

\footnotetext{
${ }^{7}$ The proton in the final state is fully constrained once all the other 4-momentum are specified.
} 

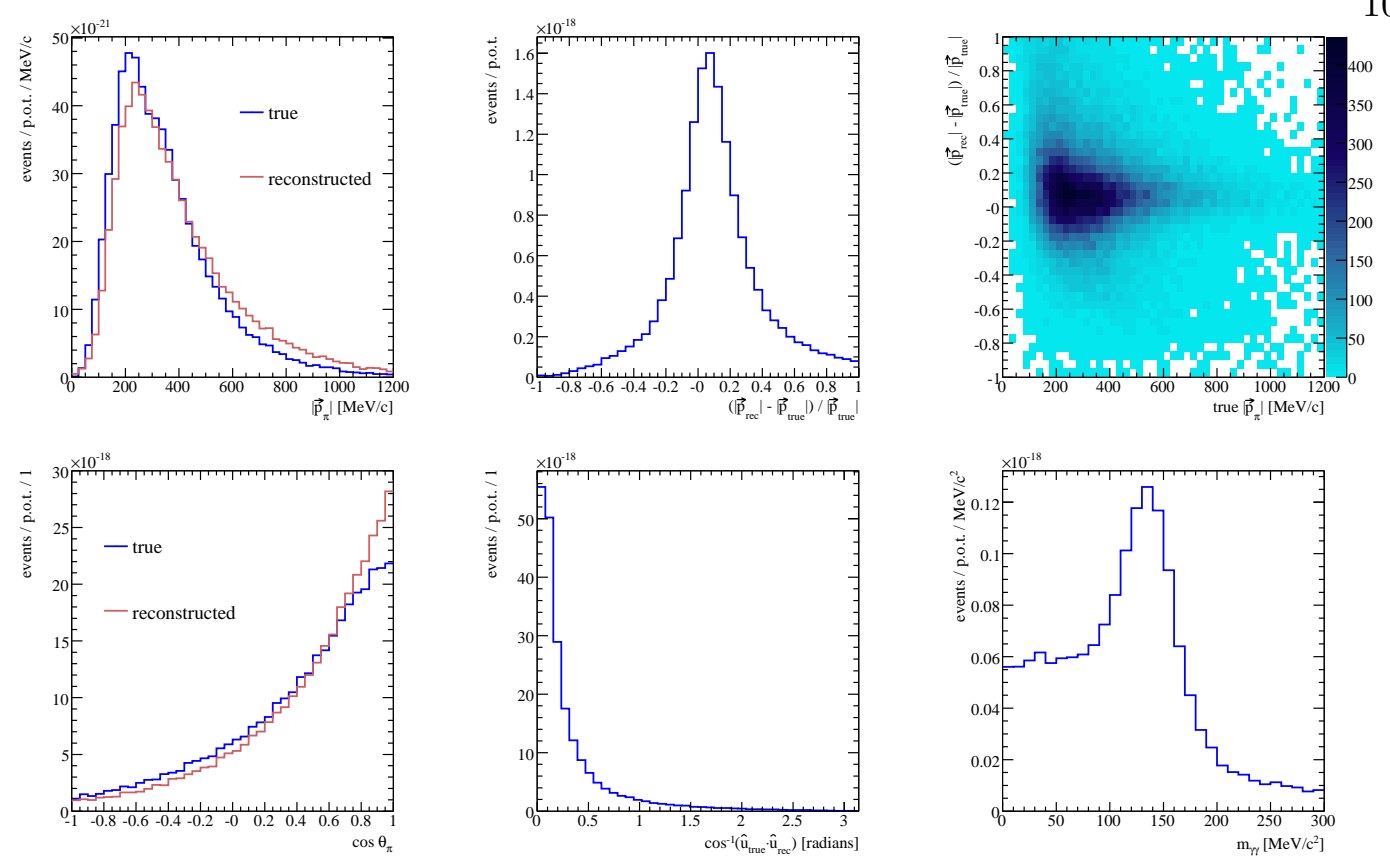

Figure 4.19: The $\pi^{0}$ fit kinematics along with the fit residuals. Top left: The magnitude of the momentum for true and reconstructed events. Top center: The momentum residual with an $14 \%$ momentum resolution in the peak. Top right: The residual vs true momentum. Bottom left: The angle wrt the beam direction for true and reconstructed events. Bottom center: The angle between the true and reconstructed directions. Bottom right: The reconstructed $\pi^{0}$ mass.

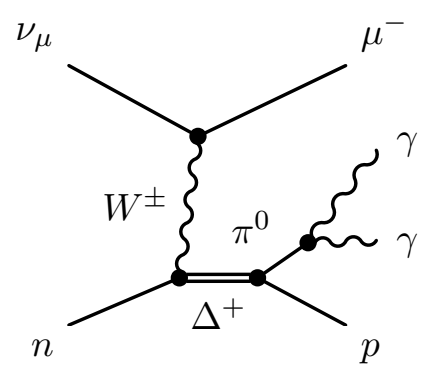

Figure 4.20: A tree level $\mathrm{CC} \pi^{0}$ Feynman diagram propagated through a $\Delta^{+}$nucleon resonance.

If these events had enough energy to produce a $W^{ \pm}$then $Q^{2}$ would be a peak at negative $W^{ \pm}$mass with a width characteristic of its lifetime. However, this convention is chosen to display the space-like nature of the boson interaction, and why $Q^{2}$ is used instead of $M_{W}^{2}$. While it is an interesting quantum mechanical fact that interactions 

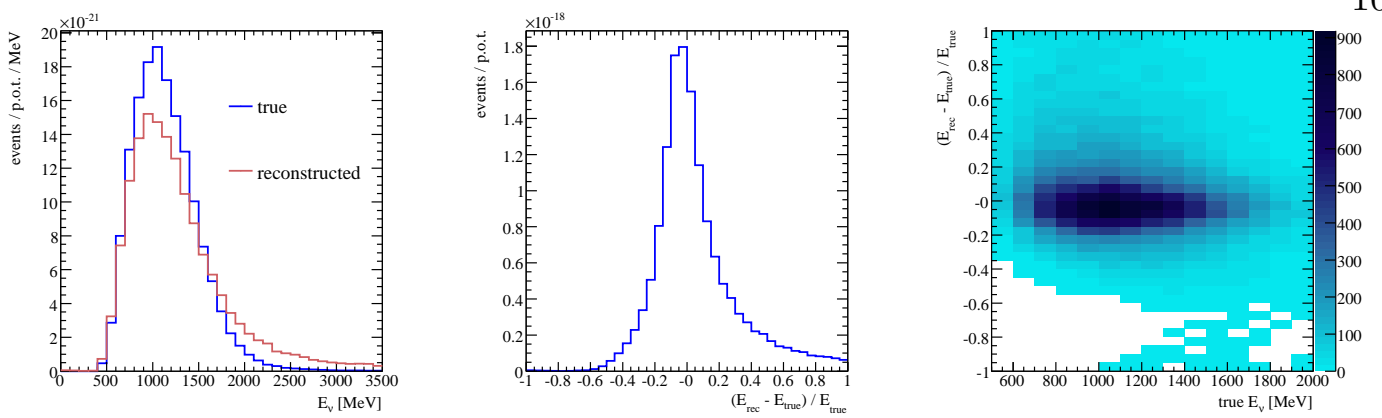

Figure 4.21: Left: The neutrino energy for true and reconstructed $\mathrm{CC} \pi^{0}$ events. Center: The neutrino energy resolution for all energies. The overall resolution is $11 \%$ in the peak. Right: The energy resolution as a function of true neutrino energy.
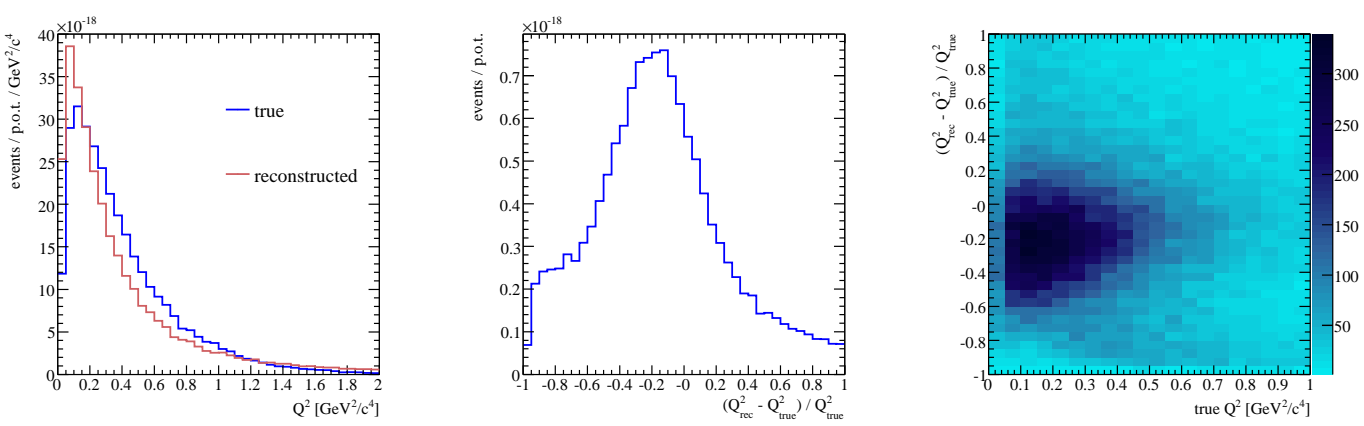

Figure 4.22: Left: The 4-momentum transfer, $Q^{2}$, for true and reconstructed $\mathrm{CC} \pi^{0}$ events. Center: The $Q^{2}$ resolution. The overall resolution is $29 \%$ in the peak. This distribution is subject to discrete misreconstruction. Right: The $Q^{2}$ resolution as a function of true $Q^{2}$. The bulk of events below -0.6 residual are from misidentifying the muon.

can exchange particles whose masses are much larger than the total energy of the event, the rate of these interactions get penalized by $M_{W}$ in the propagator.

\subsubsection{Nucleon Resonance}

Since no assumption is made about the nucleon resonance, it can be reconstructed along with everything else. The MiniBooNE MC incorporates 18 resonances below 2 $\mathrm{GeV}$ though the $\Delta$ is by far the most prominent. The resonance, $N^{*}$, is reconstructed 
as the $\pi$-nucleon mass, or equivalently as,

$$
m_{N^{*}}^{2}=m_{\mu}^{2}+m_{n}^{2}+2 m_{n}\left(E_{\mu}-E_{\mu}\right)-2 E_{\nu}\left(E_{\mu}-\left|\mathbf{p}_{\mu}\right| \cos \theta_{\nu \mu}\right)
$$

where the mass is constructed with $q$, and $p_{n}$ instead of $p_{\pi}$ and $p_{p}$. Fig. 4.23 shows the reconstructed nucleon-resonance mass. The reconstructed mass is close to the $\Delta$ mass,

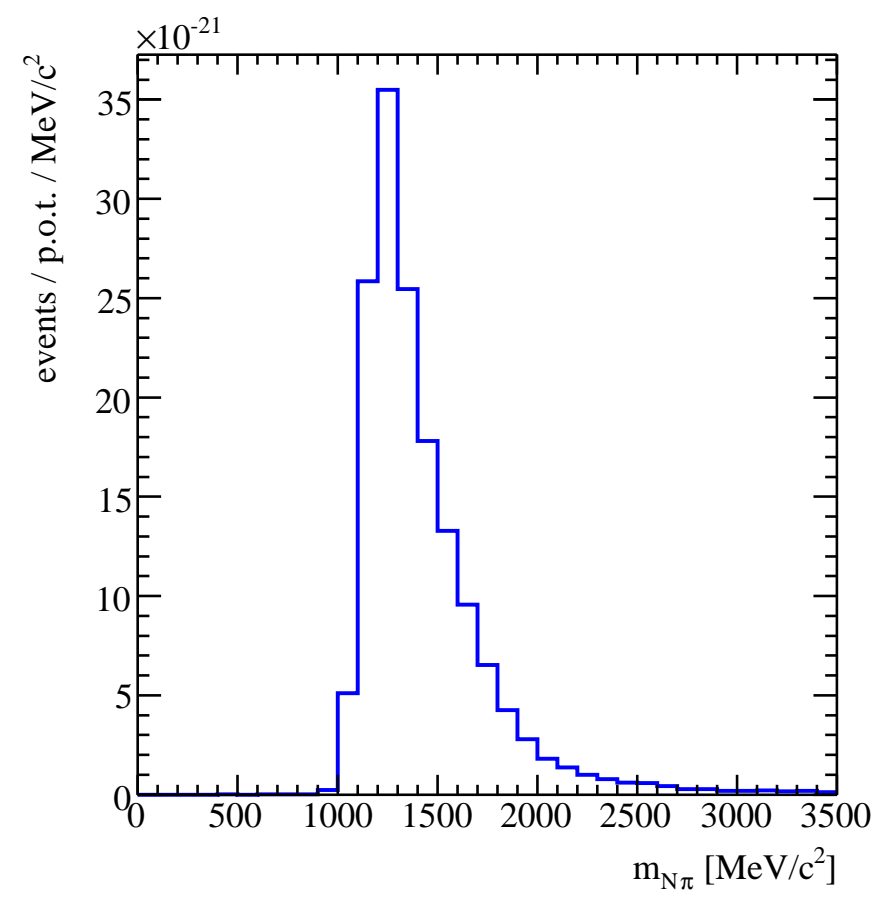

Figure 4.23: The reconstructed nucleon resonance peaks at $1240 \mathrm{MeV} / c^{2}$, very close to the $\Delta(1232)$ mass with a full width of $168 \mathrm{MeV} / c^{2}$.

with no obvious contributions from higher mass resonances.

\subsection{Rejecting Misreconstructed Events}

Ideally, the $\mathrm{CC} \pi^{0}$ fitter would correctly reconstruct all quantities (within smearing) from a $\mathrm{MC}$ sample containing only true $\mathrm{CC} \pi^{0}$ events. However, a sizable chunk of the events have particles that almost completely overlap when projected to the wall of the tank. The more PMTs that tracks share, the harder it becomes to determine 
their energies. Since the reconstructed quantities found by a misreconstructed event are completely wrong, rejecting these events is key to keeping quality reconstructed events.

To reject misreconstructed events, a cut on the smallest angle between any two of the three reconstructed tracks is made. Fig. 4.24 shows this angle for events that correctly identified the particle configuration and those that did not. Except for the

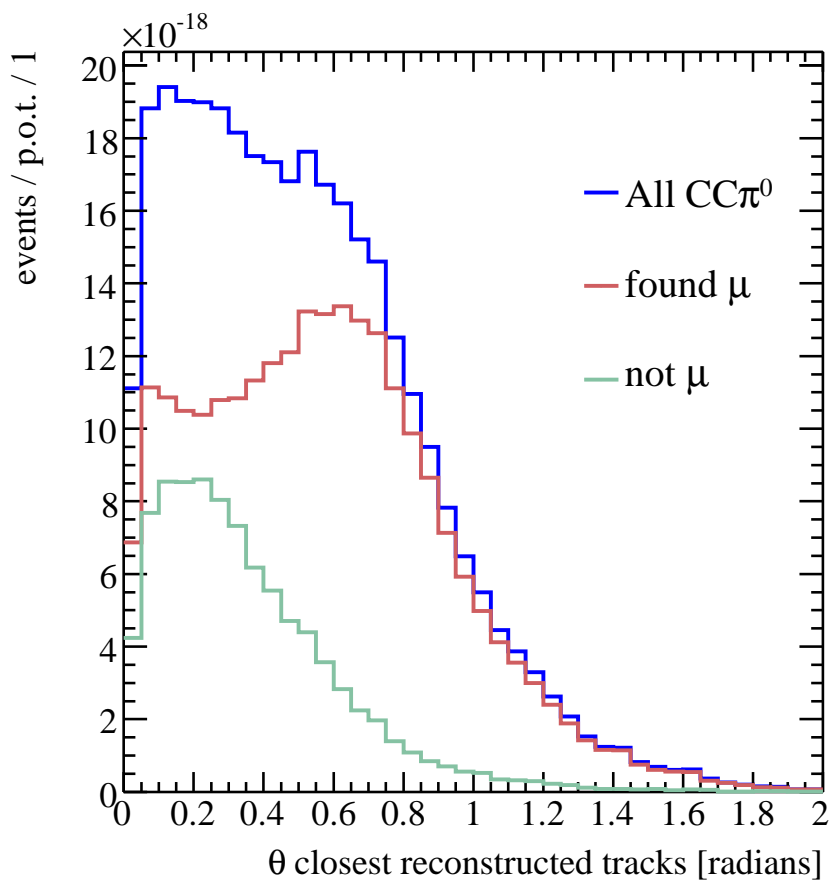

Figure 4.24: The smallest angle between the three reconstructed tracks for events that correctly identified the particle configuration (found $\mu$ ) and those that did not (not $\mu$ ).

highest energy events, there are no physics reasons for tracks to land on top of each other. Also, when they do land on top of each other, it becomes impossible to determine the track energies with any sort of accuracy. To ascertain where to place the cut an optimization is performed for rejecting misreconstructions. The criterion for rejection is,

$$
\frac{N}{\sqrt{F+N}}
$$

where $N$ are misreconstructed events, and $F$ are well reconstructed events. This crite- 
rion is not equivalent to the standard expression for maximizing a signal $(N \leftrightarrow F)$. This was chosen because the misreconstructions are fewer than the correctly reconstructed events in every bin. Maximizing the "signal" in this case, would have led to no cut. Since, the misreconstructions should be rejected, even if signal events have to be sacrificed, this background rejection criterion was used. Fig. 4.25 shows the criterion plotted versus angle. The curve maximizes the misreconstructed contribution to the left of the

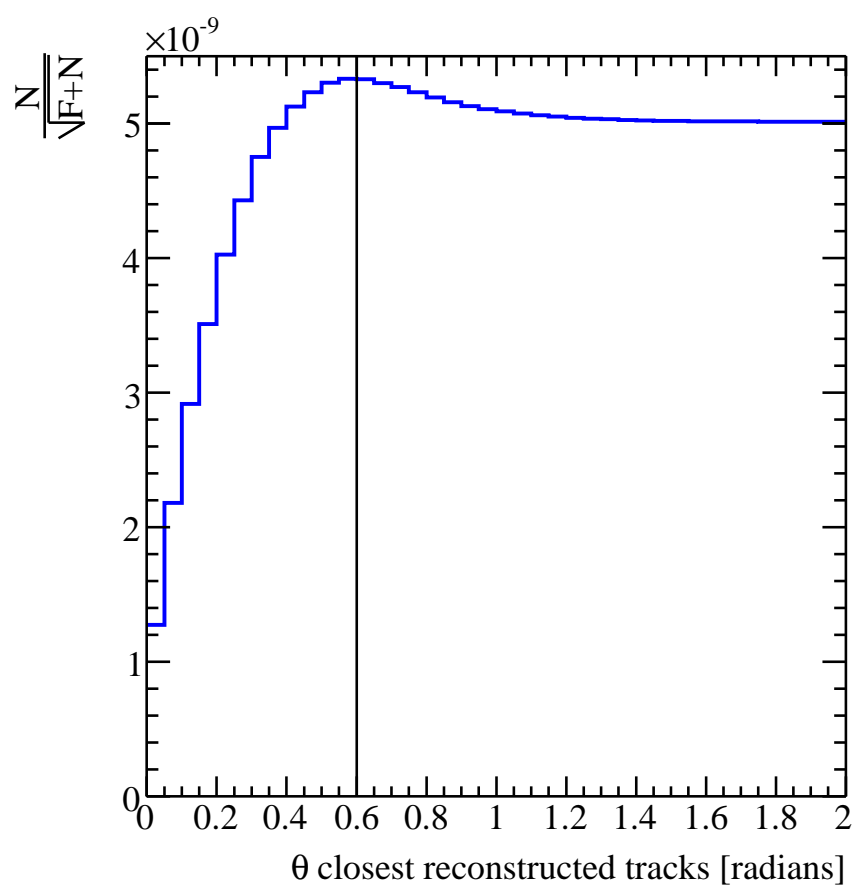

Figure 4.25: The optimization parameter $N / \sqrt{F+N}$ versus the smallest angle between reconstructed tracks. The parameter in each bin is found by integrating the numerator and denominator separately for every bin in Fig. 4.25 with angles less than itself, including itself. The line is set at the bin boundary where cutting events to the left of the line removes the optimal number of misreconstructed events.

peak. Therefore, placing the cut at the peak at 0.6 keeps all events on the right, rejecting most of the misreconstruction while still keeping a usable number of signal events. The small level of remaining misreconstruction will be addressed by the unfolding (§5.3.5).

To assess the quality of the misreconstruction rejection cut, several quantities 
from $\S 4.3$ and $\S 4.4$ will be readdressed. Fig. 4.26 shows the reconstructed muon kinetic
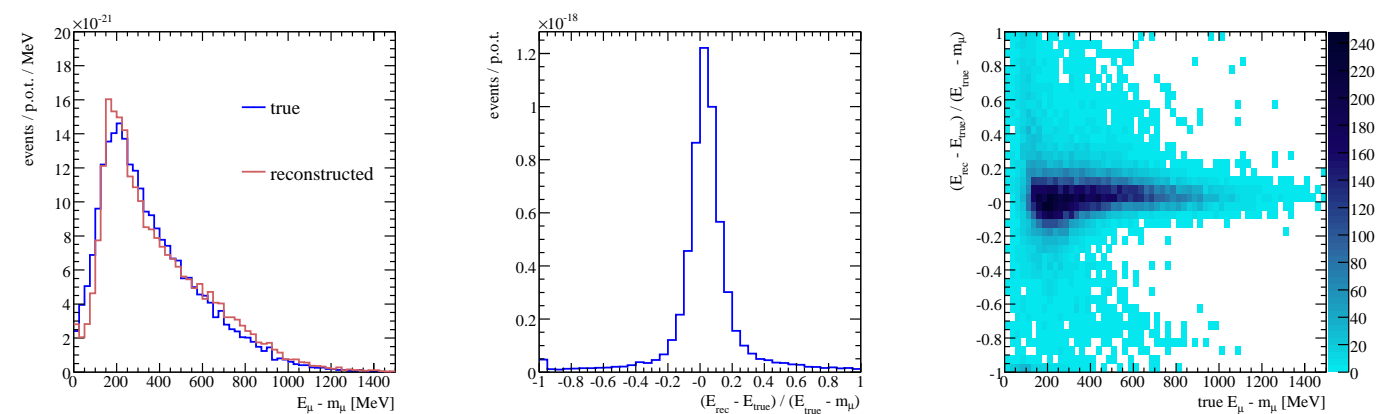

Figure 4.26: The muon kinetic energy after the misreconstruction rejection cut. The agreement between the true and reconstructed distributions is fairly remarkable. While the overall energy is unchanged, the obvious misreconstruction features found in Fig. 4.16 have been significantly reduced.

energy. The shapes of the distributions have greatly improved over their counterparts in Fig. 4.16. The energy resolution has stayed at 8\%, which was more or less expected as the peak was dominated by well reconstructed events. The distortion due to misreconstruction is now absent.

Fig. 4.27 shows the effect of the cut on both the neutrino energy and $Q^{2}$. The neutrino energy is effected only in the efficiency versus energy. Low energy events are cut because they tend to have at least one low energy track that is difficult to reconstruct. That track then lands on top of another track. High energy events are cut because either the tracks really are on top of each other due to the boost, or the $\pi^{0}$ decayed extremely asymmetrically in the lab-frame causing one of the photons to be really low energy. Also, the neutrino energy is roughly the energy of the event. Since these events are contained in the tank, all the energy is deposited into the detector. The net effect is that even if the particle types get misreconstructed, the fitter still tries to account for all the visible light and provides a good estimate of the total, or neutrino, energy. As $Q^{2}$ is completely sensitive to discrete misreconstruction, rejecting the misreconstructed events has improved the $Q^{2}$ resolution to $25 \%$ and removed the obvious low reconstructed $Q^{2}$ 

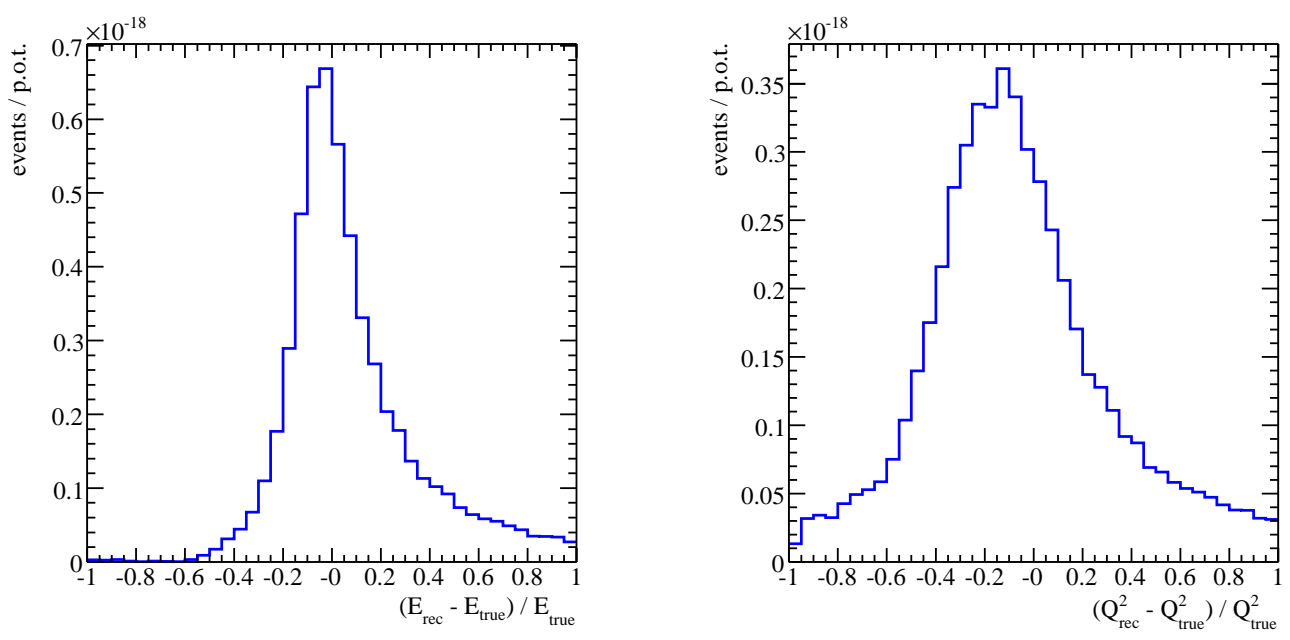

Figure 4.27: The neutrino energy and $Q^{2}$ resolutions after the misreconstruction rejection cut. The neutrino energy resolution is unaffected by the cut. The $Q^{2}$ resolution is improved to $25 \%$ overall, with the discreet misreconstruction effect seen in Fig. 4.22 at negative values significantly reduced.

misreconstruction.

Finally, Fig. 4.28 shows the effect of the cut on the pion mass. At no time during the fit is a $\pi^{0}$ mass hypothesis assumed. That the mass peaks exactly at the $\pi^{0}$ mass is a testament to the overall quality of the fitter with respect to well reconstructed events. For the cross-section measurement, a cut will be placed on the $\pi^{0}$ mass to demand the best reconstructed events in the sample, and to reject backgrounds that do not have $\pi^{0}$ in them.

\subsection{Final Thoughts}

The techniques employed to reconstruct tracks described in this chapter are by no means unique. Many different methods of reconstruction are certainly possible. However, the beauty of the method chosen was that it clearly addressed the physics of particle interactions with the detector, and was easily scalable for multi-particle events. An early form of event reconstruction used a "point-like" source approximation [81, 82, 


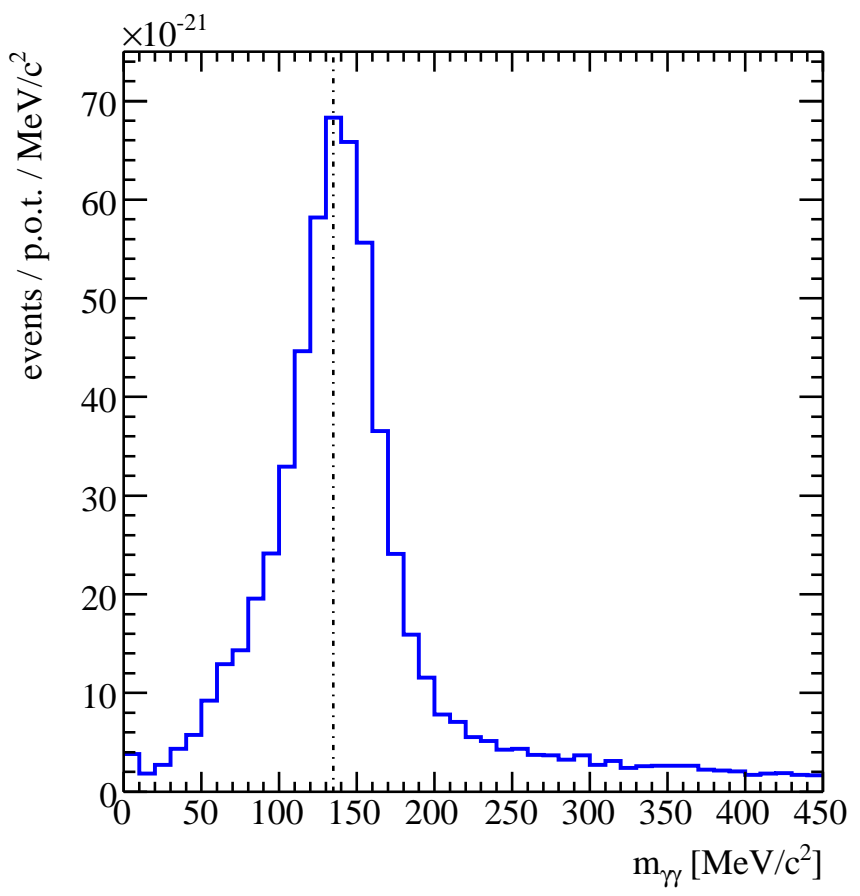

Figure 4.28: The reconstructed $\gamma \gamma$ mass after the misreconstruction rejection cut. The distribution peaks at $139 \mathrm{MeV} / c^{2}$ with a width of $47 \mathrm{MeV} / c^{2}$. The width is entirely due to smearing. The line shows the position of the world average $\pi^{0}$ mass [20].

83, 84]. This technique was well motivated in its approach as the bulk of the Cerenkov light from an event comes from the mean emission point $\left(\Delta s_{\text {mid }}\right)$. However, the main differences between muons and electrons are how they propagate through the mineral oil (see §4.1.1.2). These differences are less tangible in a point-like source approximation. The extended-track method of reconstruction was a natural evolution of the pointsource approximation and even uses the result of the point-source fit as the seed for the single-track fits.

Choosing to calculate charge and hit time probability distributions for each PMT in the detector for each set of parameters as they are floated during the fit is rather CPU intensive ${ }^{8}$. It could not be done completely on an event by event basis, so assumptions were made and tested, and quantities were tabulated beforehand to be referenced

\footnotetext{
${ }^{8}$ For this analysis the total CPU time for just the reconstruction was over $10 \mathrm{CPU}$-years.
} 
during the fit. Many simulations were performed to generate the necessary tables. The main purpose of this reconstruction was to reduce the dependence of the optical model parameters on the final physics results. This was accomplished by placing the emphasis of the reconstruction algorithm on the propagation of light in the mineral oil. This way, fundamental differences between different types of charged particles clearly stand out. On a philosophical level, the most important characteristic of the reconstruction for any analysis is to tell the difference between different types of events. For example, for the oscillation search it required the ability to not only tell the difference between muon and electron CCQE events, but also the rejection of $\mathrm{NC} \pi^{0}$ events that can fake an electron-CCQE event. For the analysis presented in this thesis, the reconstruction has to reject both muon-CCQE and $\mathrm{CC} \pi^{+}$events, which will be addressed in the next chapter. 


\section{Chapter 5}

\section{$\mathrm{CC} \pi^{0}$ Cross-sections and Analysis}

Having a comprehensive $\mathrm{CC} \pi^{0}$ event reconstruction allows for detailed measurements of $\mathrm{CC} \pi^{0}$ interaction cross-sections. A cross-section is naturally a function of every degree of freedom associated with the interaction, constrained of course by conservation of energy, momentum, and angular momentum. There are many different ways to express the measurement of a cross-section, as representation in every degree of freedom at once is impractical. The muon neutrino flux is fully characterized by neutrino energy and incident angle. The cross-section is dependent on only the neutrino energy; it is measured relative to the incident neutrino direction. As the reconstruction assumed a neutrino traveling only in the $z$-direction, the angle can smear the neutrino energy somewhat. However, as the MiniBooNE detector subtends a small solid angle with respect to the neutrino beam, this effect is negligible. Expressing the cross-section as a function of neutrino energy provides the most complete information about the probability of a $\mathrm{CC} \pi^{0}$ interaction. Expressing the $\mathrm{CC} \pi^{0}$ interaction in terms of the kinematics of the final state particles gives the probability of a given final state occurring for a given initial state. Therefore, a cross-section expressed in terms of final state kinematics is differential with respect to those variables. To recover the total probability for an interaction given a specific initial state requires integration over the final state probabilities.

The MiniBooNE suite of cross-section measurements aids in the understanding of neutrino interactions with nuclear media. With the addition of this measurement 
MiniBooNE will have measured five exclusive modes, providing the most complete description of $\nu_{\mu}$ interactions of a nuclear target at these energies. The $\mathrm{CC} \pi^{0}$ measurement is particularly interesting, as it is the only single pion production mode without a coherent interaction. Models of resonant and coherent neutrino interactions will have to be tested against the MiniBooNE measurements.

The measurements presented in this chapter are the most comprehensive measurements of $\mathrm{CC} \pi^{0}$ interactions to date. The total $\mathrm{CC} \pi^{0}$ cross-section is measured as a function of neutrino energy. Additionally, differential cross-sections are measured in terms of four final state variables $\left(E_{\mu}, \cos \theta_{\mu},\left|\mathbf{p}_{\pi^{0}}\right|\right.$, and $\left.\cos \theta_{\pi^{0}}\right)$, and one internal state variable $\left(Q^{2}\right)$.

\subsection{Previous Measurements}

The world data for measurements of the $\mathrm{CC} \pi^{0}$ cross-section at MiniBooNE beam energies come from two previous experiments: the Argonne (ANL) 12ft bubble-chamber [85, 86] and the Brookhaven (BNL) 7ft bubble chamber [87]. Both experiments used deuterium targets. High energy data from the SKAT experiment [88] $\left(E_{\nu}>3 \mathrm{GeV}\right)$ and the CERN WA25 experiment [89] $\left(E_{\nu}>7 \mathrm{GeV}\right)$ are beyond the range of interest for this measurement.

The ANL experiment measured the total $\mathrm{CC} \pi^{0}$ cross-section as a function of neutrino energy using 273 candidate events (see Fig. 5.1). The cross-section was restricted to energies less than $1.5 \mathrm{GeV}$ to reject multi- $\pi$ backgrounds. Additionally, they presented the measured $Q^{2}$ distribution but did not quote a differential cross-section. The raw events from Ref. [85] were included in the final analysis in Ref. [86], also shown in Fig. 5.1.

The BNL experiment measured the total $\mathrm{CC} \pi^{0}$ cross-section as a function of neutrino energy using 853 candidate events (see Fig. 5.1). No restriction was made on the neutrino energy and they reported values up to $3.0 \mathrm{GeV}$ neutrino energy. 


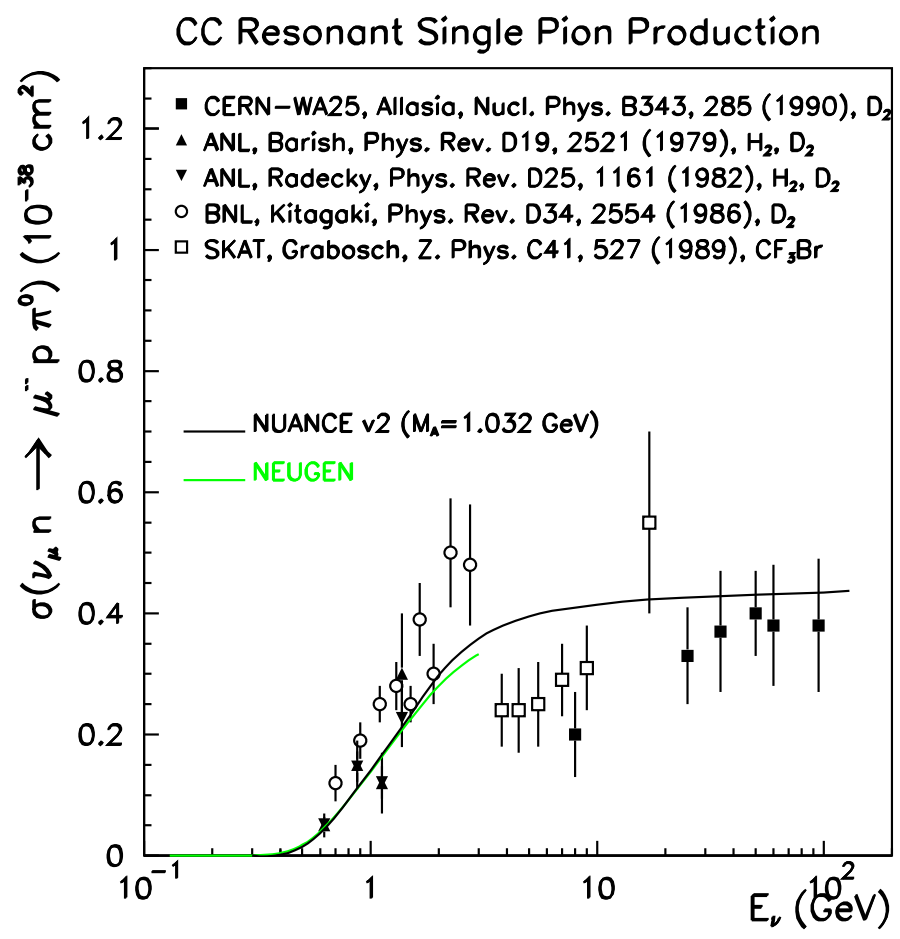

Figure 5.1: The world data for $\mathrm{CC} \pi^{0}$ production. That data are from Refs. [85, 86, 87, 88, 89]. Only the ANL and BNL experiments measured $\mathrm{CC} \pi^{0}$ interactions at MiniBooNE energies. Both those experiments used a deuterium target. The models shown underpredict the data and are the NUANCE [57] and NEUGEN [90] models.

Also shown in Fig. 5.1 are the predictions of the NUANCE [57] and NEUGEN [90] models. The data at low energy appear to be systematically higher than the model predictions. The default, dipole-form, axial mass in the Nuance model is $M_{A}=1.032$. Under a dipole assumption, it would take a higher effective axial mass to match the data from these experiments. The BNL measurement reported a higher effective axial mass of $M_{A}=1.28 \pm 0.11 \mathrm{GeV} / c^{2}$ [87]. The ANL experiment measured an axial mass, under a dipole assumption, of $M_{A} \sim 1.25 \mathrm{GeV} / c^{2}$ [86]. However, by modifying the dipole form factor as suggested by Sehgal [91],

$$
F_{A}\left(Q^{2}\right)=\frac{1}{1+Q^{2} / m_{A 1}^{2}} \exp \left(-\frac{1}{6} \frac{Q^{2} R^{2}}{1+Q^{2} / 4 M^{2}}\right)
$$

where $m_{A 1}=\sqrt{2} m_{\rho}, R^{2}=6 \mathrm{GeV}^{-2}$, and $M=M_{p}$, the axial mass has been fit to the 
quasi-elastic data and found to be $M_{A}=0.98 \mathrm{GeV} / c^{2}$. With this particular modification to the model, the axial form factor for single production is fixed.

None of the previous data at MiniBooNE energies are from nuclear targets. No experiment reports the exclusive differential cross-section with respect to $Q^{2}$. However, the Argonne experiment combines the data from the other single pion modes to extract the inclusive $d \sigma / d Q^{2}$ differential cross-section in slices of neutrino energy. Neither experiment reports differential cross-sections as functions of either the muon or the pion kinematics.

\subsection{Measuring a Cross-section}

The measurement of a cross-section is rather simple in principle. For a beam of particles incident on a target the number of interactions for a particular final state is given by

$$
N^{\text {int }}=N^{\operatorname{targs}} \sigma \otimes \Phi,
$$

where $\sigma$ is the cross-section or probability of an interaction, $N^{\text {targs }}$ is the number of targets, and $\Phi$ is the incident flux into the volume containing the targets. This formula assumes both that the target material is of uniform density, and that the particle beam is not subject to energy losses in the target material. However, since mineral oil is fairly incompressible, and neutrinos interact only weakly, these assumptions are very strong. Of greater concern is the stability of the oil's density with respect to temperature changes. The thermal expansion coefficient of Marcol 7 was measured to be $6.1(4) \cdot 10^{-4} \mathrm{~K}^{-1}[38]$. It would take a temperature change of $16.4 \mathrm{~K}$ to cause the oil to expand by $1 \%^{1}$. Therefore, density variations with temperature are not a great concern. Additionally, as $N^{\text {int }}$ is measured by the experiment, and $N^{\text {targs }}$ and $\Phi$ are chosen (or measured) by the experimenter, enough information is present to solve for the cross-section.

\footnotetext{
${ }^{1}$ The variation of the MiniBooNE oil over the course of the neutrino run was between $3-5 \mathrm{~K}$ [92].
} 
The situation expressed in Eqn. 5.2 is slightly more complicated when expressed in terms of initial and final state variables. The most general expression for Eqn. 5.2 is given by

$$
N^{\mathrm{int}}=N^{\mathrm{targs}} \int \frac{\partial^{N} \sigma\left(x_{1} \ldots x_{M}\right)}{\partial y_{1} \ldots \partial y_{N}} \frac{\partial^{M} \Phi}{\partial x_{1} \ldots \partial x_{M}} d \mathbf{x} d \mathbf{y}
$$

where $x_{i}$ are initial state variables, $y_{i}$ are final state variables, $d \mathbf{x}$ and $d \mathbf{y}$ are integrals over each full set of variables (e.g. $d \mathbf{x}=d x_{1} \ldots d x_{M}$ ). A cross-section is a function of the initial state variables, which are decided before the interaction, and differential in terms of the final state variables.

To extract the cross-section in terms of initial state variables, all final state variables are integrated over and Eqn. 5.3 becomes

$$
N^{\mathrm{int}}=N^{\operatorname{targs}} \int \sigma\left(x_{1} \ldots x_{M}\right) \frac{\partial^{M} \Phi}{\partial x_{1} \ldots \partial x_{M}}, d \mathbf{x}
$$

where the dependence on the final state kinematic variables is integrated out. The rate of interactions also depends on the flux, which determines the set of neutrino energies. The total flux is given by

$$
\Phi=\int \frac{\partial^{M} \Phi}{\partial x_{1} \ldots \partial x_{M}} d \mathbf{x}
$$

and a slice of the flux is

$$
\Phi_{i}=\int_{E_{i}} \frac{\partial^{M} \Phi}{\partial x_{1} \ldots \partial x_{M}} d \mathbf{x}
$$

where $E_{i}$ is a bin of neutrino energy. Dividing the interactions by the flux and rearranging the expression becomes

$$
\langle\sigma\rangle_{\Phi}=\frac{1}{\Phi} \int \sigma\left(x_{1} \ldots x_{M}\right) \frac{\partial^{M} \Phi}{\partial x_{1} \ldots \partial x_{M}} d \mathbf{x}=\frac{N^{\text {int }}}{\Phi N^{\operatorname{targs}}},
$$

where $\langle\sigma\rangle_{\Phi}$ is recognized as the flux-averaged total cross-section. Choosing the only flux variable, the neutrino energy, the binned cross-section is

$$
\sigma_{i}\left(E_{\nu}\right)=\frac{N_{i}^{\mathrm{int}}\left(E_{\nu}\right)}{\Phi_{i} N^{\mathrm{targs}}}
$$


where the dependence on all other factors has been integrated out, $N_{i}^{\text {int }}$ counts the interactions in bin $i$ of $E_{\nu}$, and $\Phi_{i}$ is the flux slice over that interval. There is no way to get around the flux dependence of the interaction rate for a cross-section measurement. However, if small enough slices of the neutrino energy are used, then the flux-averaging occurs over a small interval in bins of the measurement. If the flux varies slightly over this interval, the dependence effectively cancels out and the cumbersome flux-averaging notation is dropped.

For cross-sections in terms of final state variables the integrals in Eqn. 5.3 are performed over the initial state energies,

$$
N^{\text {int }}=N^{\text {targs }} \Phi \int\left\langle\frac{\partial^{N} \sigma}{\partial y_{1} \ldots y_{N}}\right\rangle_{\Phi} d \mathbf{y}
$$

where the entire flux is averaged over. As the flux averaging is independent of the final state derivatives, the expression for the cross-section becomes

$$
\int \frac{\partial^{N}\langle\sigma\rangle_{\Phi}}{\partial y_{1} \ldots y_{N}} d \mathbf{y}=\frac{N^{\mathrm{int}}}{\Phi N^{\mathrm{targs}}}
$$

For bins of a single final state variable the differential cross-section is

$$
\left(\frac{\partial \sigma}{\partial y}\right)_{i}=\frac{N_{i}^{\operatorname{int}}(y)}{\Phi N^{\operatorname{targs}} \Delta y_{i}}
$$

where $\Delta y_{i}$ is the bin width. The cumbersome flux-averaging notation has been dropped, though in this case the flux averaging is over the whole flux. The flux averaging can be removed if the single differential cross-section is expressed as a function of neutrino energy. Following the procedure of this section, the single differential cross-section as a function of neutrino energy is

$$
\left(\frac{\partial \sigma\left(E_{\nu}\right)}{\partial y}\right)_{i j}=\frac{N_{i j}^{\mathrm{int}}\left(y, E_{\nu}\right)}{\Phi_{j} N^{\mathrm{targs}} \Delta y_{i}}
$$

where the differential cross-section is averaged over the flux bin $\Phi_{j}$. Differential crosssections can be constructed from any number of final state kinematic variables. 
To estimate the number of signal events in a bin of a particular variable in data, a couple of corrections have to be applied. The measured number of events contains backgrounds, is affected by detector smearing, and suffers from cut efficiencies. The first correction is a subtraction of the expected backgrounds. The backgrounds are either measured, or estimated from the MC. An "unfolding" correction ( 55.3 .5$)$ is performed to address smearing effects and failings of the assumptions used in the reconstruction. The final correction to the measured event rate is an efficiency correction to restore events lost through the series of cuts. Therefore, the number of inferred interactions in a bin of a variable is given by

$$
N_{i}^{\text {int }^{\prime}}=\frac{\sum_{j} U_{i j}\left(N_{j}^{\mathrm{int}}-B_{j}^{\mathrm{int}}\right)}{\epsilon_{i}},
$$

where $N_{j}^{\text {int }}$ is the number of measured interactions, $B_{j}^{\text {int }}$ is the expected background, $U_{i j}$ is the unfolding matrix, and $\epsilon_{i}$ is the cut efficiency. The inferred number of events is used in the total cross-section and the differential cross-section equations derived above (Eqns. 5.8, 5.11, and 5.12).

\subsection{The $\mathrm{CC} \pi^{0}$ Measurement}

The difficulties in measuring the $\mathrm{CC} \pi^{0}$ cross-section are two-fold. The biggest difficulty was already addressed by the event reconstruction. Having an accurate event reconstruction allows for the measurement of not only the total cross-section, but additionally the cross-section with respect to the final state particles, and inferred internal states (e.g. $\left.Q^{2}\right)$.

The next difficulty arises from the fact that $\mathrm{CC} \pi^{0}$ is a relatively rare mode overall ( $\sim 4 \%$ of the total rate). This implies that isolating a relatively pure sample of $\mathrm{CC} \pi^{0}$ events, reducing model dependence and the effect of systematic errors, will be rather difficult. Once a pure sample is isolated, the remaining backgrounds must be subtracted out. Ideally, the backgrounds would be constrained by measurements within the same 
data set. This can be accomplished, in this case, for CCQE and $\mathrm{CC} \pi^{+}$events. However, the Monte Carlo (MC) is trusted for the remaining backgrounds. The uncertainties on those backgrounds can be rather large and therefore negatively affect the quality of the measurement.

Once these difficulties are overcome, it is a simple matter to extract both total and differential cross-sections. This measurement details: the cut efficiencies, used to correct event rate; the unfolding, used to correct out reconstruction assumptions and smearing; the neutrino flux, used to remove the beam dependence from the rate to extract the total cross-section, or flux-average the differential cross-sections results. The sources of systematic uncertainties are explored along with the philosophy used to facilitate the error propagation and extraction.

The cross-section measurement method will be fully explored for the neutrino energy total cross-section measurement with additional details discussed for the differential measurements addressed as needed. Finally, the cross-section results will be presented.

\subsubsection{Defining the Signal}

When dealing with nuclear media, a distinction must be made between neutrinonucleus interactions and the interaction of the final state particles with the target nucleus. An ideal measurement is one where the initial state can be fully reconstructed. However, as the pion can reinteract with the nucleus a couple of things can happen that can affect the measurement. The pion can scatter, absorb, or even charge exchange $\left(\pi^{+} n \rightarrow \pi^{0} p\right)$. These interactions are collectively referred to as final state interactions (FSI). For example, an initial state $\mathrm{CC} \pi^{+}$event can have its $\pi^{+}$absorbed in the nucleus and have the final state look identical to a CCQE event. Additionally, as that same $\pi^{+}$ can charge exchange into a $\pi^{0}$, the final state can look identical to a $\mathrm{CC} \pi^{0}$ event. On an event by event basis, these FSI events are indistinguishable from neutrino-nucleon 
interaction $\mathrm{CCQE}$ and $\mathrm{CC} \pi^{0}$ events without FSI. Experimentally these are defined to be "observable" events and the rates can be significantly different from the nucleonlevel interactions. For the case of observable $\mathrm{CC} \pi^{0}$, the raw contribution nucleon level $\mathrm{CC} \pi^{0}$ according to NUANCE is $68.3 \%$. The bulk of the remaining nucleon level modes contribute $20.7 \%$ (7.2\%) from $\mathrm{CC} \pi^{+}(\mathrm{CCQE})$ events with the remaining $3.8 \%$ made up from NC modes and multi-pion $\mathrm{CC}$ modes.

At these energies nucleon-level CCQE interactions dominate the total event rate. Therefore, while nucleon-level $\mathrm{CC} \pi^{+}$events make it into the observable CCQE sample, the uncertainties in FSI are manageable and the nucleon-level $\mathrm{CC} \pi^{+}$can be subtracted out leaving a nucleon-level CCQE measurement intact.

However, for the case of $\mathrm{CC} \pi^{0}$, as the rate for nucleon-level $\mathrm{CC} \pi^{+}$are much greater than the rate for nucleon-level $\mathrm{CC} \pi^{0}$, the amount of nucleon-level $\mathrm{CC} \pi^{+}$can not be subtracted with a reasonable level of uncertainty. Therefore, the measurements presented in this chapter are of observable $\mathrm{CC} \pi^{0}$ and not nucleon-level $\mathrm{CC} \pi^{0}$. From an experimental point of view, an "observable" $\mathrm{CC} \pi^{0}$ measurement is the most useful for predicting rates in a detector as the FSI are folded into the measurement. Observable measurements are as model-independent as can be measured; they are what the experiment sees from the interaction that happened.

\subsubsection{Cuts}

To isolate a sample of $\mathrm{CC} \pi^{0}$ events from the $O\left(10^{6}\right)$ neutrino interactions recorded at MiniBooNE is no small task. Roughly $40 \%$ of the event rate are CCQE events. As charged-current muon events are tagged by their subsequent muon decays, $\mathrm{CC} \pi^{0}$ events look very similar to CCQE events. A series of cuts are performed to isolate $\mathrm{CC} \pi^{0}$ events from the overwhelming CCQE sample. These cuts are applied in two phases. The first are a series of precuts designed to isolate both CCQE and $\mathrm{CC} \pi^{0}$ from neutral-current and $\mathrm{CC} \pi^{+}$events. The goal here is to reduce the sample to something more manageable 
to run the $\mathrm{CC} \pi^{0}$ fitter on. The second series of cuts are both for data quality and to reject backgrounds using the information gained from the $\mathrm{CC} \pi^{0}$ fit. The summary of the cut efficiencies and purities are displayed in Table 5.1 at the end of this section.

\subsubsection{Event Tagging}

The first level of event sorting is performed by grouping clusters (in time) of PMT hits in an event. All data from the detector's PMTs are triggered $4.6 \mu$ s before the neutrino beam reaches the detector. The beam occurs over a $1.6 \mu$ s interval, defined to be the beam window, while data collection occurs for a total of $19.2 \mu \mathrm{s}$. As discussed in $\S 4.1 .2$, a particle track produces all of its light over a short period of time. All of that light falls within the 200 ns window accepted by the discriminators. A group of ten or more hits with time spacings less than 10 ns between the hits, with at most two spacings less than $20 \mathrm{~ns}$, is called a "subevent." The first subevent is always created by the initial neutrino interaction and is required to fall in the beam time window. All subsequent subevents are due to electrons from stopped muon decays (Michel decays). The long muon lifetime $(\tau \simeq 2.2 \mu \mathrm{s})$ implies that the muon tends to decay outside of the beam window. Also, the trigger window only collects data from an early portion of the muon decays. Additionally, some muon decays are lost from either $\mu^{-}$capture on nuclei, or very early "clobber" decays that occur during the first subevent window. Fig. 5.2 shows an example of a 2 subevent event. Events can be classified in their ideal scenarios based on their expected number of subevents: 


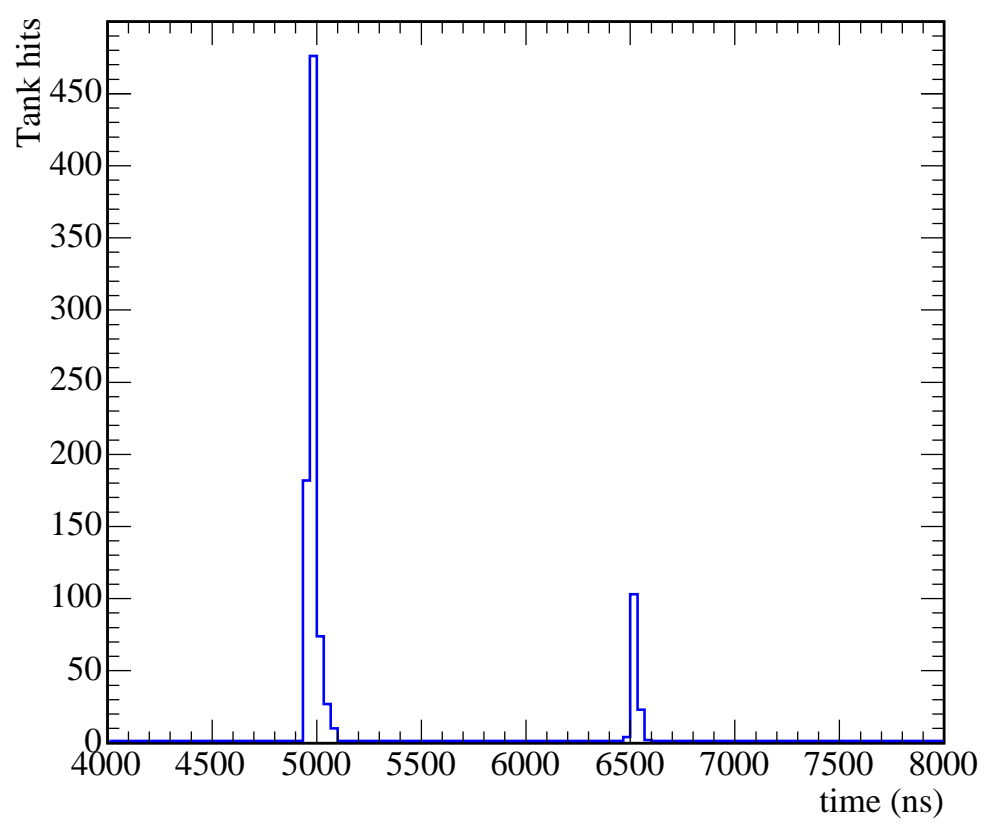

Figure 5.2: For a single neutrino interaction the number of PMT hits is plotted as a function of the time of the hit. The first cluster falls within the $1.6 \mu$ beam window and is called the first "subevent." The second cluster is due to a stopped muon decay and is called the second "subevent."

\begin{tabular}{cc} 
interaction & subevents \\
\hline \hline $\mathrm{CCQE}$ & 2 \\
$\mathrm{CC} \pi^{+}$ & 3 \\
$\mathrm{NCEL}$ & 1 \\
$\mathrm{NC} \pi^{0}$ & 1 \\
$\mathrm{CC} \pi^{0}$ & 2
\end{tabular}

The $\mathrm{CC} \pi^{+}$sample has three subevents because of the additional Michel electron provided by the muon decay from the pion decay. The NC current modes listed have no muons and therefore no Michel electrons. Unfortunately, CCQE makes up most of the 2 subevent sample with the fraction of expected $\mathrm{CC} \pi^{0}$ at $5 \%$ of the events. Clearly, additional filtering needs to be done to isolate a relatively pure sample of $\mathrm{CC} \pi^{0}$ events. 
Additionally, cuts on the number of PMT hits in the main tank and the veto region are performed on each subevent to reject events that either enter or exit the main tank. These cuts are set to isolate a pure sample of contained events, rejecting cosmic-ray induced events, and stopped muon decays where the muon entered the main portion of the tank before the beam trigger, but decayed during the beam time window. Stopped muon decays, with a maximum electron energy of $52.8 \mathrm{MeV}$, always produce fewer than 200 PMT hits in the main tank. Additionally, events that enter the veto region tend to have more than 6 hit veto PMTs. This means that a few simple cuts on PMT hits can be made to isolate a pure sample of neutrino interactions during the beam window for 2 subevent events:

- Tank hits $>200$ for the first subevent. This cut removes cosmic events that entered the tank prior to the beam window, whose decay occurred during the beam window.

- Tank hits $<200$ for the second subevent. This cut demands that the second subevent is consistent with a stopped muon decay.

- Veto hits $<6$ for both subevents. This cut demands that both subevents are contained within the main tank volume to be consistent with a neutrino interaction inside the tank.

Clearly, the efficiency of these cuts will vary with muon energy as energetic muons tend to exit the tank.

The MC prediction for the number of neutrino interactions in data is $1,019,534$. Observable CCQE constitute $44.1 \%$ of the total number of interactions with observable $\mathrm{CC} \pi^{0}$ at $3.6 \%$. The 2 subevent cut increases the CCQE and $\mathrm{CC} \pi^{0}$ fractions to $58.5 \%$ and $5.0 \%$ respectively, mainly because of the rejection of 3 subevent $\mathrm{CC} \pi^{+}$events and 1 subevent NC events. The efficiencies are $52.8 \%$ and $55.7 \%$ respectively. The addition of the PMT hits cuts on both subevents increase the fractions to $5.6 \%(70.6 \%)$ for 
$\mathrm{CC} \pi^{0}(\mathrm{CCQE})$ events, and reduce the efficiencies to $38.2 \%(38.9 \%)$. With these first series of basic cuts, designed to broadly tag the event types, roughly $60 \%$ of the interactions have been lost. Most of the rejected events are not reconstructable.

\subsubsection{Event Filter}

To define the $\mathrm{CC} \pi^{0}$ event sample, the number of CCQE events need to be significantly reduced. A CC $\pi^{0}$ event consists of a sharp muon ring and two fuzzy electron-like rings. Compared to a CCQE event, a $\mathrm{CC} \pi^{0}$ event should therefore, look a lot "fuzzier." The single-track $\mu / e$ fits are extremely quick and are performed on the first subevent of every event. The comparisons of the single-track likelihood fits should show $\mathrm{CC} \pi^{0}$ events to be more electron-like than muon-like. Fig. 5.3 shows the $\mu / e$ likelihood ratio for CCQE events and $\mathrm{CC} \pi^{0}$ events as a function of fit muon kinetic energy. The filter is optimized to reduce the CCQE contamination, while keeping most of the remaining $\mathrm{CC} \pi^{0}$ events. This cut reduces the CCQE efficiency to $1.6 \%$ and reduces the $\mathrm{CC} \pi^{0}$ slightly to $32.5 \%$. Most important, the fraction of $\mathrm{CC} \pi^{0}$ events has increased to $29.2 \%$ while the CCQE events have reduced to $17.8 \%$. The largest background is now due to $\mathrm{CC} \pi^{+}$events with a fraction of $31.0 \%$.

\subsubsection{Fiducial Cut}

The fiducial volume is set in the calculation of the efficiency. This volume is set to include as much uniform detector material as possible. Any differences between the true fiducial volume and the reconstructed fiducial are corrected out in the efficiency. For this analysis, the fiducial volume is defined as a $550 \mathrm{~cm}$ radius sphere. The final-fit reconstructed radius is used to cut events with radius greater than $550 \mathrm{~cm}$. The cut is designed to reject events that occurred near the tank walls, or within materials other than mineral oil. The fiducial volume cut is not really expected to change the purities much, but it does reduce the efficiencies. The efficiencies after this cut are $27.9 \%, 1.5 \%$, 


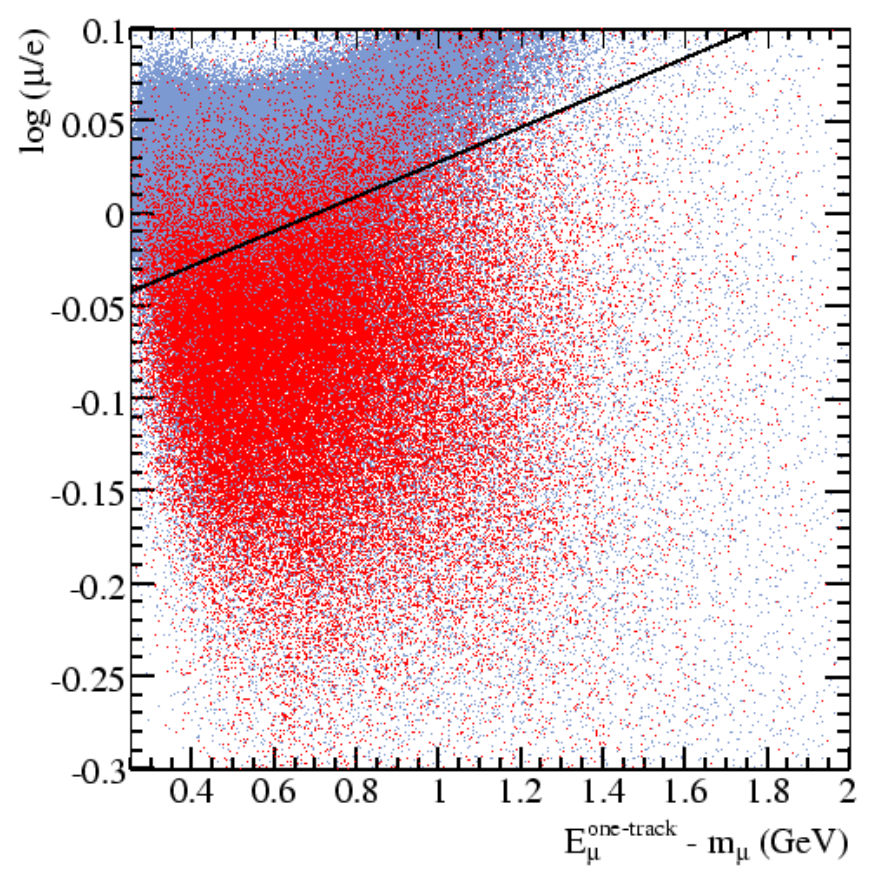

Figure 5.3: The one-track fits likelihood ratio as a function of fit muon kinetic energy. CCQE events are shown in blue and populate the top of the plot. Overlayed are $\mathrm{CC} \pi^{0}$ events, in red, which tend to look more electron-like. The line, defining the $\mathrm{CC} \pi^{0}$ filter, shows the optimum position for a cut to keep $\mathrm{CC} \pi^{0}$ events and reject CCQE events.

and $5.9 \%$ for $\mathrm{CC} \pi^{0}$, CCQE, and $\mathrm{CC} \pi^{+}$respectively.

\subsubsection{Misreconstruction Cut}

To reject misreconstructed events the cut described in $\S 4.5$ is used. The cut was optimized solely on signal events in the MC. Fig. 5.4 shows the cut for data and central value MC. This plot is inclusive of the MC shown in Fig. 4.24. As correctly identified 


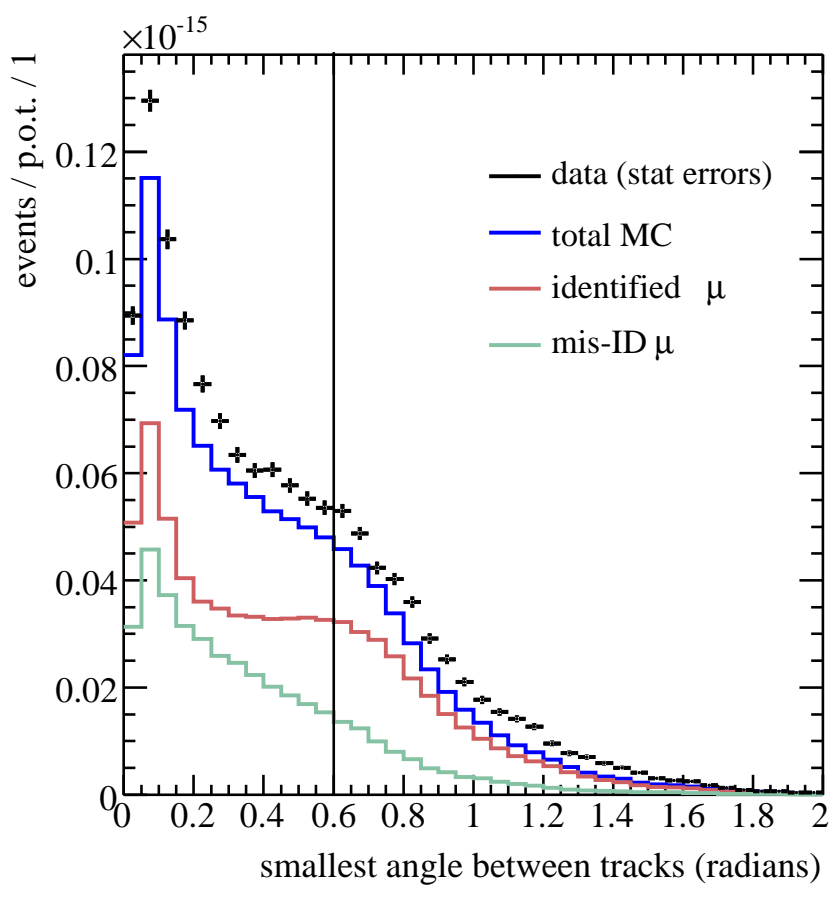

Figure 5.4: The smallest angle between the three reconstructed tracks for both data (black) and central value MC (blue). Backgrounds are included in the MC. The MC is also broken up into events that correctly identified the muon (red), according to the particle-ID, and those that do not (green). Data are shown with statistical errors. Both samples have fiducial, tank and veto hits, and $\mathrm{CC} \pi^{0}$ event filter cuts applied. The black line shows the position of the cut to reject misreconstructed events found in $\S 4.5$. Events to the left of the line are cut.

events plateau, the level of misreconstruction decreases continuously with angle. The optimization chose the edge of the plateau to place the cut, consequently rejecting a large number of events. However, the sacrifice in statistics is worth the gain in quality of the reconstruction. Additionally, as many backgrounds do not have three tracks, this cut has the added benefit of increasing the $\mathrm{CC} \pi^{0}$ event purity. The efficiency of $\mathrm{CC} \pi^{0}$ events is $10.3 \%$, with every other mode below $3 \%$. The fractions are $38.1 \%, 32.2 \%$, and $13.4 \%$ for $\mathrm{CC} \pi^{0}, \mathrm{CC} \pi^{+}$, and CCQE respectively. 


\subsubsection{Likelihood Cut}

The $\mathrm{CC} \pi^{0}$ fitter was constructed under the assumption that there is a $\mu^{-}$and a decayed $\pi^{0}$ in the final state of the interaction. Events that do not have $\pi^{0}$ in the final state, and therefore, usually no energetic photons, should reconstruct with a poorer likelihood in the fitter than events with a $\pi^{0}$ in the final state. Background $\pi^{0}$ decays can either occur at the event vertex, or through interactions with the mineral oil, can occur away from the event vertex. As the fitter assumes that the $\pi^{0}$ decay occurs at the event vertex, this class of backgrounds should look slightly worse in the fit likelihood than signal events. Fig. 5.5 shows the optimization of the likelihood cut. As expected, back-
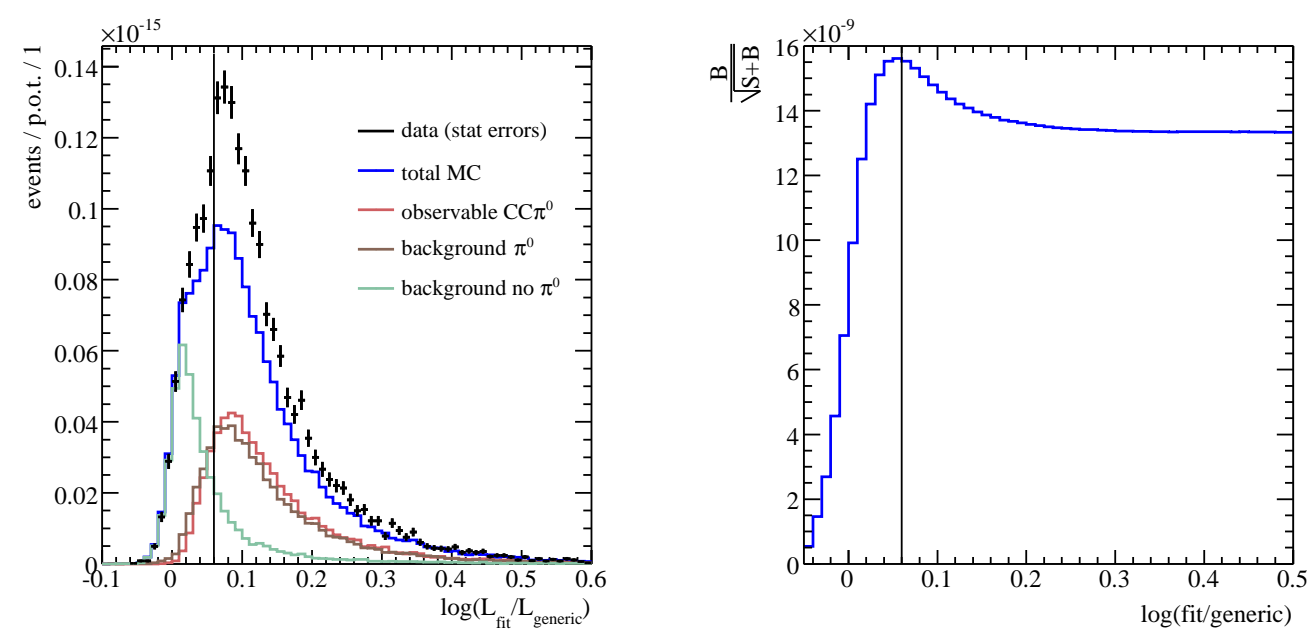

Figure 5.5: Left: The logarithm of the ratio of the final fit likelihood compared with the generic three-muon fit likelihood for both data (black) and central value MC (blue). The MC is split into signal events (red), background events with $\pi^{0}$ (brown), and backgrounds without $\pi^{0}$ (green). The line optimizes the cut to reject backgrounds without $\pi^{0}$ while preserving signal, events to the left are cut. Right: Non- $\pi^{0}$ background rejection cut optimization. Backgrounds with $\pi^{0}$ are not including in the optimization as they look extremely similar to signal.

ground events without $\pi^{0}$ pile up at small likelihood ratio (many below 0), while signal and backgrounds with $\pi^{0}$ only improve with the final fit (ratio $>0$ ). The distribution of backgrounds with $\pi^{0}$ show a similar shape to the signal, however, the distribution 
is shifted to slightly smaller values showing the effect of possibly having a disjoint $\pi^{0}$ vertex, or additional tracks in the event. The signal fraction improves to $47.0 \%$ with a reduction in both $\mathrm{CC} \pi^{+}$and $\mathrm{CCQE}$ event fractions to $24.4 \%$ and $10.7 \%$ respectively. The efficiency for signal is $8.9 \%$ with the next largest efficiency of CCmulti- $\pi$ at $2.3 \%$. All other efficiencies are below $1 \%$.

\subsubsection{Pion Mass Cut}

By reconstructing the photons from the $\pi^{0}$ decay, the full four-vector of the $\pi^{0}$ is reconstructed. The reconstructed $\gamma \gamma$ mass should therefore be able to distinguish events with $\pi^{0}$ final states from those without. Fig. 5.6 shows the cut optimization on
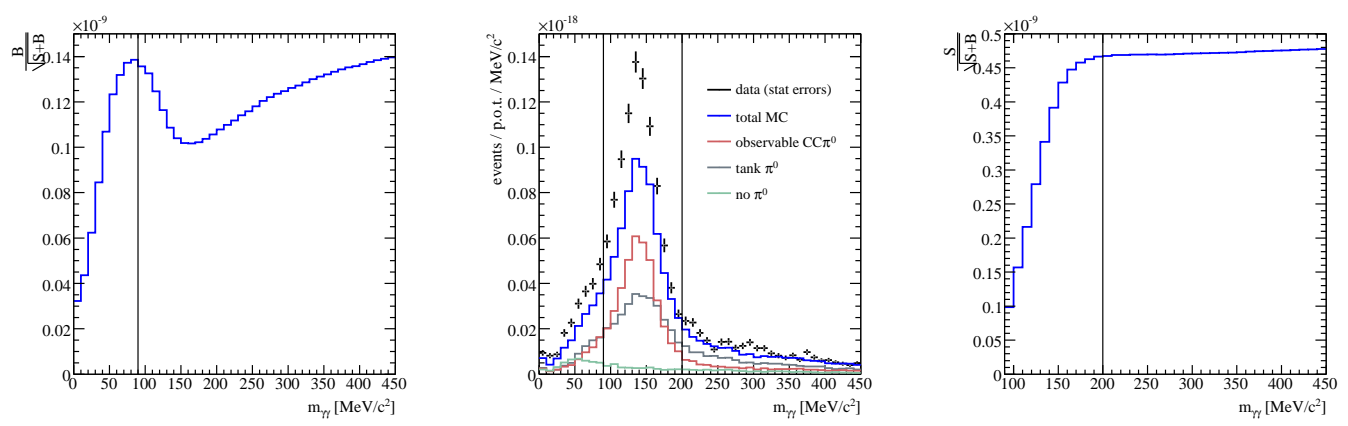

Figure 5.6: Center: The reconstructed $\gamma \gamma$ mass. The MC is separated into signal (red), backgrounds with $\pi^{0}$ in the final state (brown), and backgrounds without $p i^{0}$ (green). The lines represent the location of the optimized cuts, with the region around the obvious $\pi^{0}$ mass kept. Left: Optimization of the low mass cut to reject non- $\pi^{0}$ events from signal. Events to the left of the line are cut. Right: Optimization of the signal over all backgrounds. Events to the right of the line are cut.

the reconstructed $\gamma \gamma$ mass about the known $\pi^{0}$ mass. The low mass cut is optimized to reject non- $\pi^{0}$ backgrounds, as they tend to pile up at low reconstructed mass. The high mass cut is optimized to maximize signal over all backgrounds. As this optimization does not converge, the cut is placed at the start of the plateau, where loosening the cut would increase the number of events but reduce signal purity and quality of event reconstruction. This cut attempts to reject backgrounds and accept signal with high 
quality reconstruction. The total efficiency after this cut for $\mathrm{CC} \pi^{0}$ events is $6.4 \%$. The purity is $56.7 \%$. The largest single background is from observable $\mathrm{CC} \pi^{+}$events and has a $22.5 \%$ fraction.

\subsubsection{Cut Summary}

The cuts that have been described in this section were applied to isolate a relatively pure sample of observable $\mathrm{CC} \pi^{0}$ events while demanding a quality reconstruction. Each cut was optimized after the application of all prior cuts. Table 5.1 summarizes the effect of each cut. Additionally, the table shows the expected NUANCE nucleon-level fractions of the modes that contribute to observable $\mathrm{CC} \pi^{0}$ events. The contributions are fairly constant with the addition of each cut, and show that the final fraction for nucleonlevel $\mathrm{CC} \pi^{0}$ in observable $\mathrm{CC} \pi^{0}$ is $67.1 \%$. The remainder is mostly from nucleon-level $\mathrm{CC} \pi^{+}$events.

\subsubsection{Event Rate}

The first piece of the cross-section is the measured event rate. Fig. 5.7 shows the measured event rate for neutrino energy for both data and MC after cuts. The first thing to notice is the normalization difference where data is greater than MC across the whole energy range. Additionally, the shapes of the distributions are slightly different. Neither of these effects is unexpected as the total inclusive event rate in data is roughly $20 \%$ larger than MC, varying somewhat across the modes. However, as the simulation covers the entire range of interest for this measurement, the $\mathrm{MC}$ will still be a useful tool in extracting the cross-section and calculating systematic errors.

\subsubsection{Backgrounds}

The next step towards estimating the number of interactions is to subtract off the expected backgrounds from the measured event rate. Ideally, the best way to ac- 


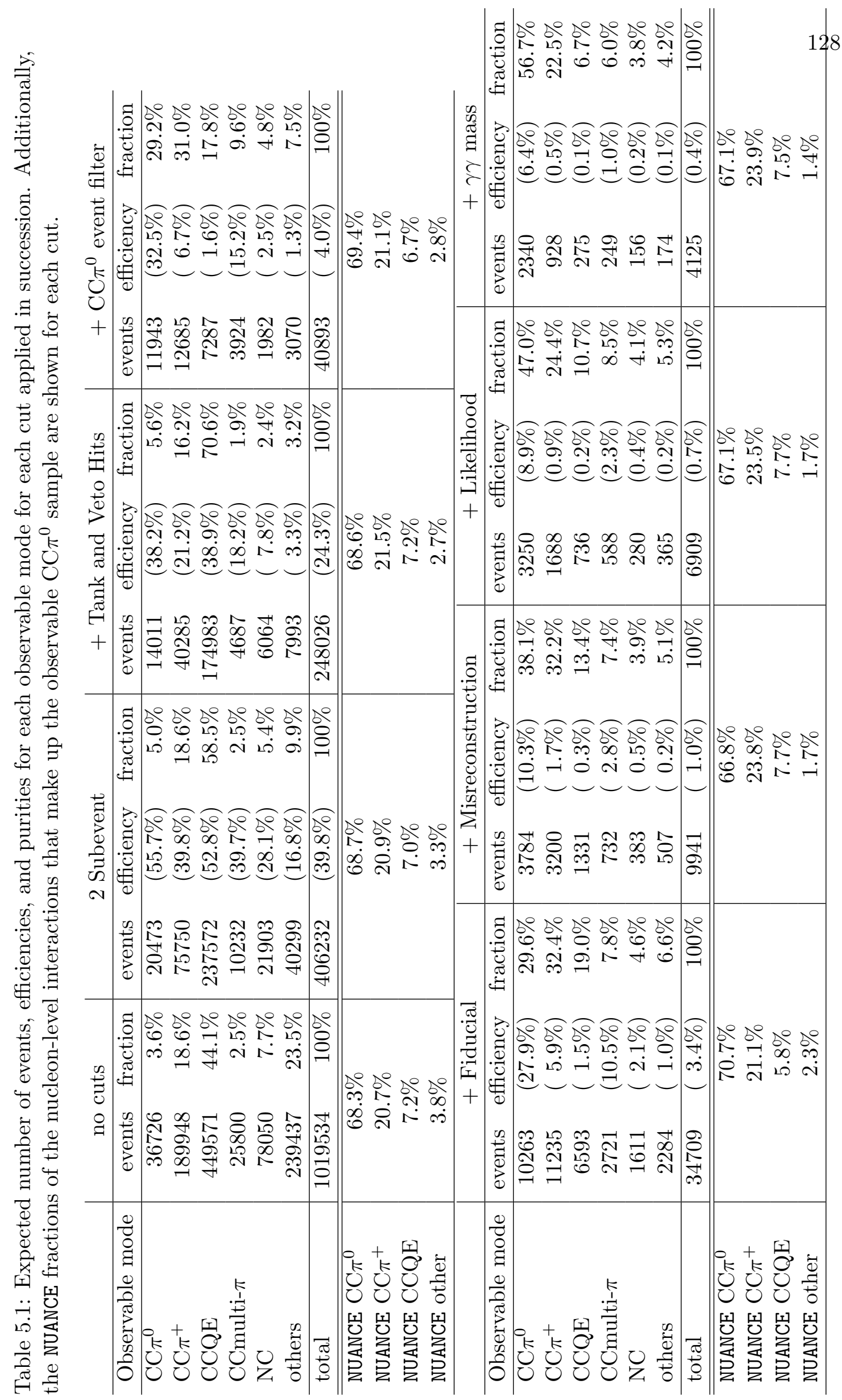




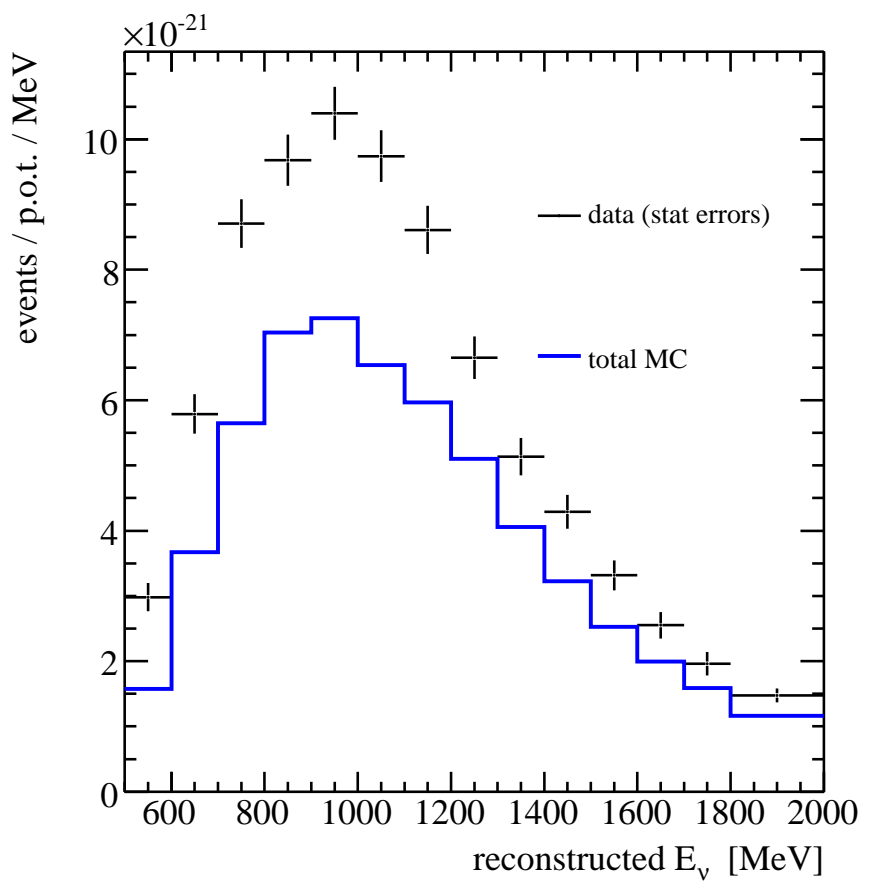

Figure 5.7: The measured event rate in neutrino energy for data and total MC. Data is shown with Poisson statistical error bars.

complish this would be to set the level of expected backgrounds based on measurements performed on other samples of the total data set. This is done for the largest single background, the observable $\mathrm{CC} \pi^{+}$, which contributes to about half of the total background. This contribution is mainly due to the fact that, as will be discussed in $\S 5.15$, while ideal $\mathrm{CC} \pi^{+}$events typically have 3 subevents, but the small fraction of $\mathrm{CC} \pi^{+}$events that either clobber, charge-exchange, or suffer $\pi^{+}$absorption, end up directly into this sample. For the remaining backgrounds, the MC is trusted to provide their amounts. However, as the uncertainties on the production of these backgrounds are rather large per mode, the uncertainties associated with these backgrounds will adequately cover any possible normalization difference not currently constrained by the data.

Fig. 5.8 shows the signal and background separated by observable mode in the reconstructed $\gamma \gamma$ mass. Observable $\mathrm{CC} \pi^{+}$events make up the largest background. 


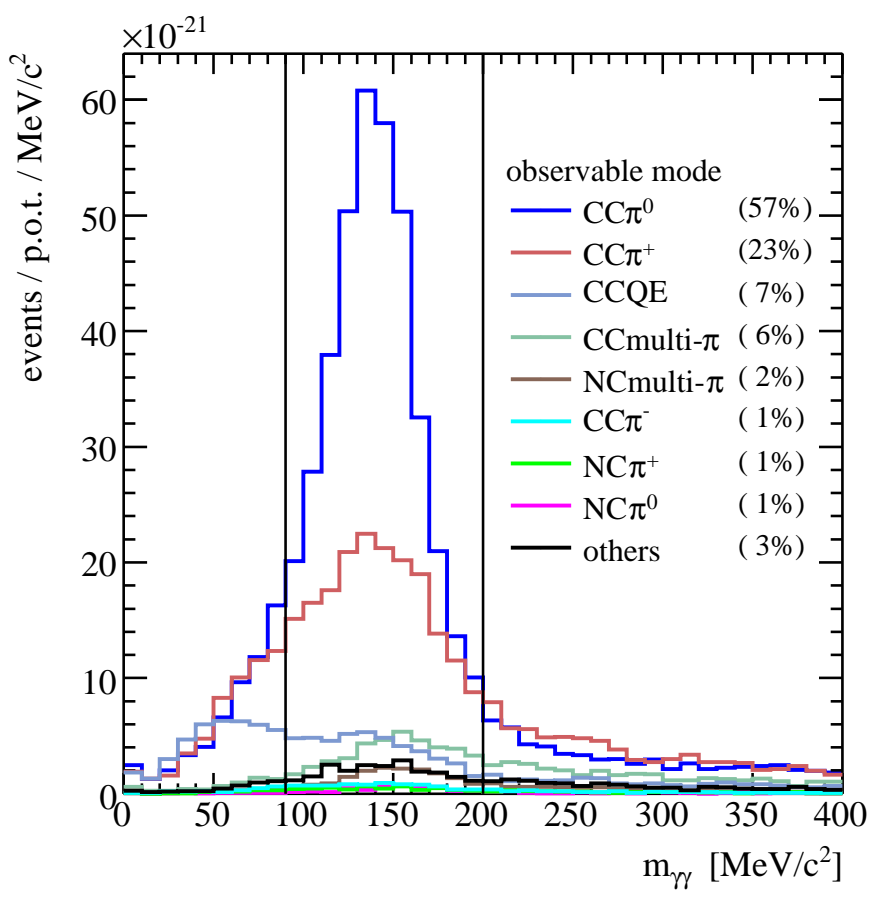

Figure 5.8: The reconstructed $\gamma \gamma$ mass by observable mode. Each mode is represented after all cuts (including the mass cut). The observable $\mathrm{CC} \pi^{0}$ dominates the sample with $\mathrm{CC} \pi^{+}$as the largest source of background. The $\mathrm{CC} \pi^{+}$events have been reweighted according to the method described in $\S 5.3 .4 .1$.

These events are reweighted to the measured production in the MiniBooNE data by the method described in $§ 5.3 .4 .1$. The remaining backgrounds, CCQE included, are subtracted based on the predictions from the MC. Eventually, CCQE production may be set to the level measured in data, however, as the CCQE measurement is a neutrino-nucleon interaction measurement, the method for estimating an observable mode overlaps the nucleon-level reweighting and must be accounted for. The remaining backgrounds are an agglomeration of modes with pions in the final state, CCQE that leaked in to the sample, and some DIS. The "other" modes are a mix of events from non- $\nu_{\mu}$ interactions and final states with other mesons including the occasional kaon. There is no single contribution that accounts for more than one percent of the total background. Table 5.2 summarizes the breakdown of the remaining background by observable mode after all 
Table 5.2: The remaining background after all cuts by observable mode. Percentages are relative to the total amount of background.

\begin{tabular}{l|c} 
Observable background & Contribution \\
\hline \hline $\mathrm{CC} \pi^{+}$ & $53 \%$ \\
$\mathrm{CCQE}$ & $16 \%$ \\
$\mathrm{CCmulti}-\pi$ & $14 \%$ \\
$\mathrm{NCmulti}-\pi$ & $5 \%$ \\
$\mathrm{CC} \pi^{-}$ & $2 \%$ \\
$\mathrm{NC} \pi^{+}$ & $2 \%$ \\
$\mathrm{NC} \pi^{0}$ & $2 \%$ \\
other & $7 \%$
\end{tabular}

cuts. These backgrounds are subtracted from the reconstructed distributions prior to unfolding and efficiency corrections.

\subsubsection{1 $\quad \mathrm{CC} \pi^{+}$Backgrounds}

By tying the level of observable $\mathrm{CC} \pi^{+}$interactions to measurements within the MiniBooNE data set, the uncertainty on the production of observable $\mathrm{CC} \pi^{+}$is highly constrained. This is due to the fact that the observable $\mathrm{CC} \pi^{+}$that leak into this sample are a small sample of the total $\mathrm{CC} \pi^{+}$interactions in the detector. Two measurements of observable $\mathrm{CC} \pi^{+}$are used to reweight the cross-sections for $\mathrm{CC} \pi^{+}$production in this sample. The differential observable $\mathrm{CC} \pi^{+}$cross-section with respect to $\pi^{+}$momentum as a function of energy and the total observable $\mathrm{CC} \pi^{+}$cross-section as a function of neutrino energy. The measurements are detailed in Ref. [71] and the values used were taken from tables therein. Fig. 5.9 shows the ratio of data over MC for the observable $\mathrm{CC} \pi^{+}$cross-sections. As the momentum of the $\pi^{+}$is important for determining the kinematics of $\pi^{+}$interactions in the mineral oil, it was chosen as the most appropriate measurement. However, there were regions of momentum phase space that were not measured and these are filled in in the ratio by using the total cross-section ratio. This is for two reasons: the differential cross-section did not report values for $\pi^{+}$momentum 


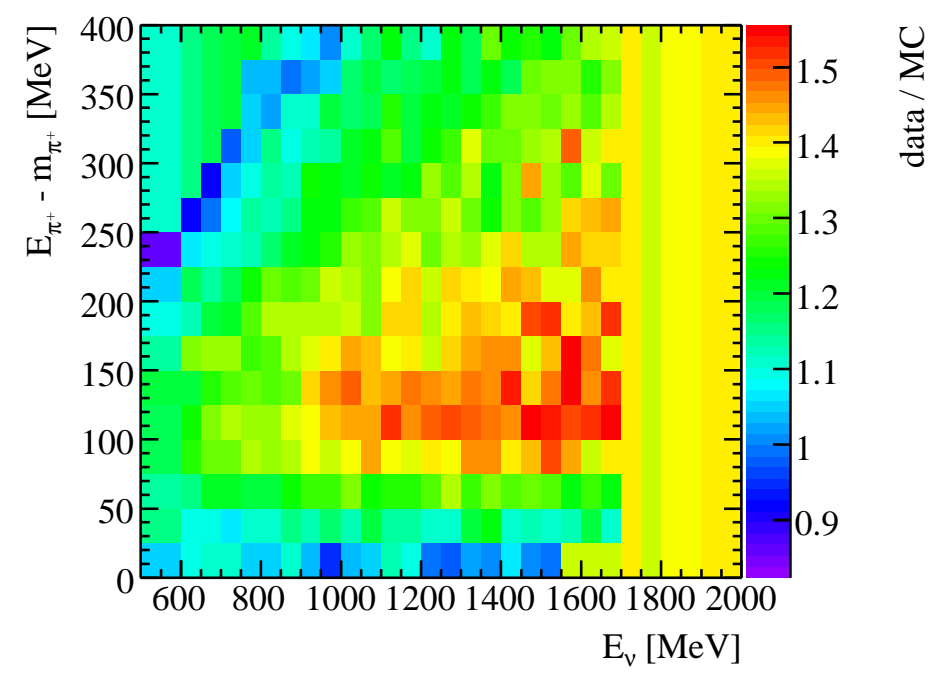

Figure 5.9: Ratio of data over MC for the observable $\mathrm{CC} \pi^{+}$cross-section as measured in Ref. [71]. This ratio forms the reweighting that is applied to each observable $\mathrm{CC} \pi^{+}$ event in the $\mathrm{CC} \pi^{0}$ event sample.

above $400 \mathrm{MeV}$, and also cut off the neutrino energy at $1700 \mathrm{MeV}$ where the total cross-section extends to $2000 \mathrm{MeV}$. This cross-section was not previously measured and it is therefore not surprising that regions of phase space differed from predictions by over $40 \%$.

Rather than reproduce all of the MC utilizing these new cross-section measurements, it is equivalent to take events already generated (assuming they cover the phase space) and reweight each event by the values of the ratio based on their true neutrino energy and $\pi^{+}$momentum.

\subsubsection{Unfolding}

After the background has been subtracted the event rate needs to be corrected for detector smearing effects and failings of the initial assumptions. The method used 
to generate the unfolding matrix is described in detail in Appendix A. The problem is reduced to finding a matrix that maps a measured distribution of events to the underlying true distribution that caused them. This matrix is generated using MC signal events. Ideally, the matrix should be completely independent from the MC true distribution used to generate the matrix. However, those methods are inherently unstable. The method chosen is based on Bayes' theorem and has a slight dependence, or bias, on the prior distribution. The matrix is the Bayesian probability $[93,94]$ that a given true event of some variable and bin, came from a reconstructed event of the same variable but different bin and is given by

$$
P\left(t_{i} \mid r_{j}\right)=\frac{P\left(r_{j} \mid t_{i}\right) P\left(t_{i}\right)}{\sum_{k} P\left(r_{j} \mid t_{k}\right) P\left(t_{k}\right)}
$$

where $t_{i}$ is bin $i$ of the true distribution, and $r_{j}$ is bin $j$ of the reconstructed distribution. This matrix is formed by generating a reconstructed vs. true 2-D histogram and dividing each row of the matrix by the number of events in that row. Fig. 5.10 show the unfolding matrix for neutrino energy. The bias comes about as the shape of the true distribution matters to some extent. However, the shape dependence is fairly weak, more so if the true distribution does not vary significantly across the bin. The inferred true distribution in data is given by,

$$
I_{i}=\sum_{j} P\left(t_{i} \mid r_{j}\right) \times r_{j}
$$

This method can be extended for any number of dimensions. The method for doing this is detailed in Appendix A. Each differential cross-section is unfolded additionally in neutrino energy. The reason for this will be addressed in $\S 5.6$.

The purpose of the unfolding is to remove the smearing associated with detector effects, misreconstructions, and weak assumptions associated with extracting physics quantities from the reconstruction.

This removes the dependence of the experiment on the measured central value result, placing most of the detector dependence in the systematic errors. The final results 


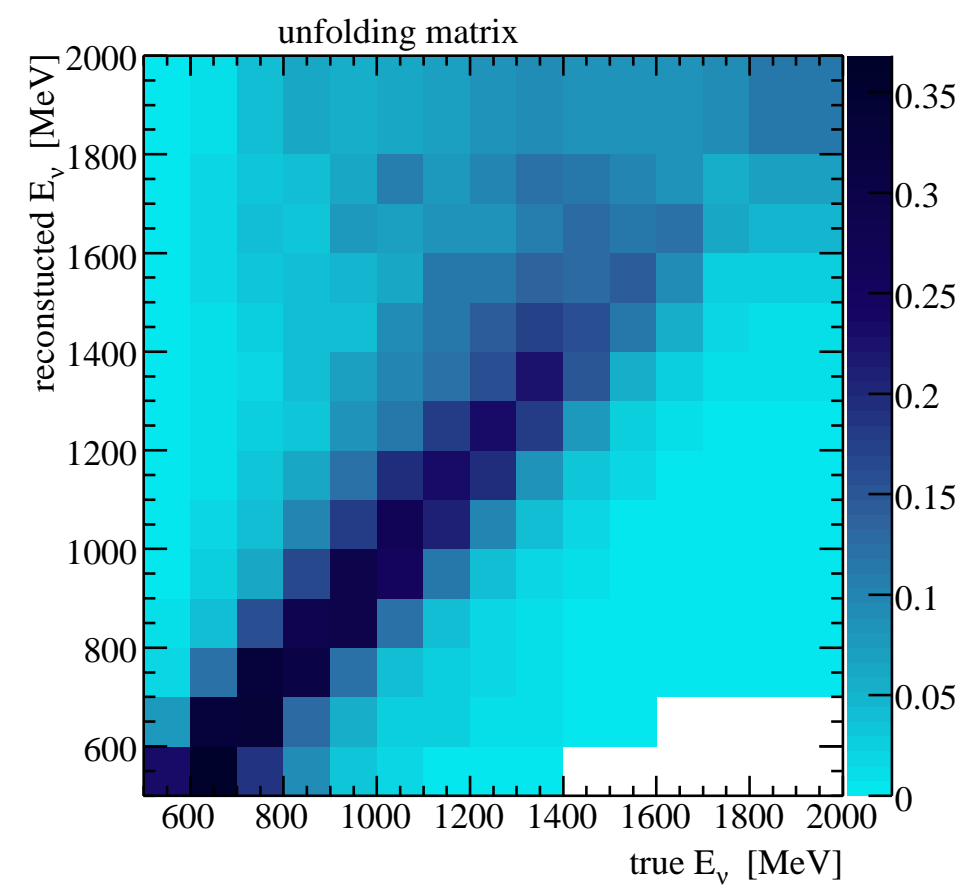

Figure 5.10: The Bayesian unfolding matrix for neutrino energy.

are mostly independent of the MiniBooNE detector, and nuclear modeling effects.

\subsubsection{Efficiency}

After the event rate is unfolded it must be corrected to account for signal events that were lost by the cuts to the number of expected interactions within the fiducial volume. For a continuous distribution the number of events passing cuts for a given set of parameters $\mathbf{x}$, is given by

$$
\frac{d N}{d \mathbf{x}}^{\text {cuts }}=\epsilon(\mathbf{x}) \frac{d N}{d \mathbf{x}}
$$

where $\epsilon(\mathbf{x})$ is the efficiency, and $\frac{d N}{d \mathbf{x}}$ is the uncut number of events in the fiducial volume. As these quantities are necessarily discretized the efficiency is integrated over a bin of a given quantity, or quantities, the number of events in a bin after cuts is

$$
N_{x_{i}}^{\text {cuts }} \equiv \int_{x_{i}} \frac{d N^{\text {cuts }}}{d \mathbf{x}} d \mathbf{x}=\int_{x_{i}} \epsilon(\mathbf{x}) \frac{d N}{d \mathbf{x}} d \mathbf{x}
$$


where the integral is performed over the whole space in all variables except the variable of interest $x$. Also, as $x$ is discretized, the integral is over the bin $i$. While this is shown for a single variable $x$, it is completely general for multi-dimensional distributions. The bin efficiency is defined to be

$$
\epsilon_{x_{i}} \equiv \frac{\int_{x_{i}} \epsilon(\mathbf{x}) \frac{d N}{d \mathbf{x}} d \mathbf{x}}{\int_{x_{i}} \frac{d N}{d \mathbf{x}} d \mathbf{x}},
$$

this quantity is measured in the MC by taking the number of events in bin $i$ of a true distribution after cuts and dividing by the sample before cuts. The bin efficiency is the true efficiency averaged over the shape of the true distribution in that bin. Clearly, if the shape varies significantly in that bin, then the bin efficiency becomes a poor estimator of the true efficiency in that bin, implying that larger bins have more possibility of bias in bin efficiency. To see this explicitly let $n$ represent the number of events in data. Then the correction to the number of events in data is given by,

$$
n_{x_{i}}^{\prime}=\frac{1}{\epsilon_{x_{i}}} n_{x_{i}}^{\text {cuts }}
$$

Assuming for the sake of argument that the total number of events in data, $n_{x_{i}}$, and the data efficiency, $\epsilon^{\prime}(\mathbf{x})$, are known, then

$$
n_{x_{i}}^{\text {cuts }}-\epsilon_{x_{i}} n_{x_{i}}=\int_{x_{i}}\left\{\epsilon^{\prime}(\mathbf{x})-\frac{\int_{x_{i}} \epsilon(\mathbf{y}) \frac{d N}{d \mathbf{y}} d \mathbf{y}}{\int_{x_{i}} \frac{d N}{d \mathbf{y}} d \mathbf{y}}\right\} \frac{d n}{d \mathbf{x}} d \mathbf{x}
$$

will equal zero in a perfect measurement. The set of variables $\mathbf{y}$ are the same as $\mathbf{x}$; they are distinguished by the integrals. The difference in the integral is small if both the data and MC distributions are relatively flat across adjacent bins and the efficiencies are the similar. As neither $\frac{d n}{d \mathbf{x}}$ nor $\epsilon^{\prime}(\mathbf{x})$ are known quantities, this calculation can not be done. However, this does illustrate a point about counting measurements that require efficiency corrections. The fewer bins used in a measurement, the more bias introduced through the efficiency correction. Ideally, the number of bins is set based on a number of different factors; the number of events in a bin, the resolution in the variables in question, and the smoothness of the efficiency distribution. By binning a distribution, 
it is impossible to avoid bias completely in the measurement. In most cases, there does not seem to be any other choice.

Fig. 5.11 shows the bin efficiency vs. neutrino energy after all cuts. As low-energy

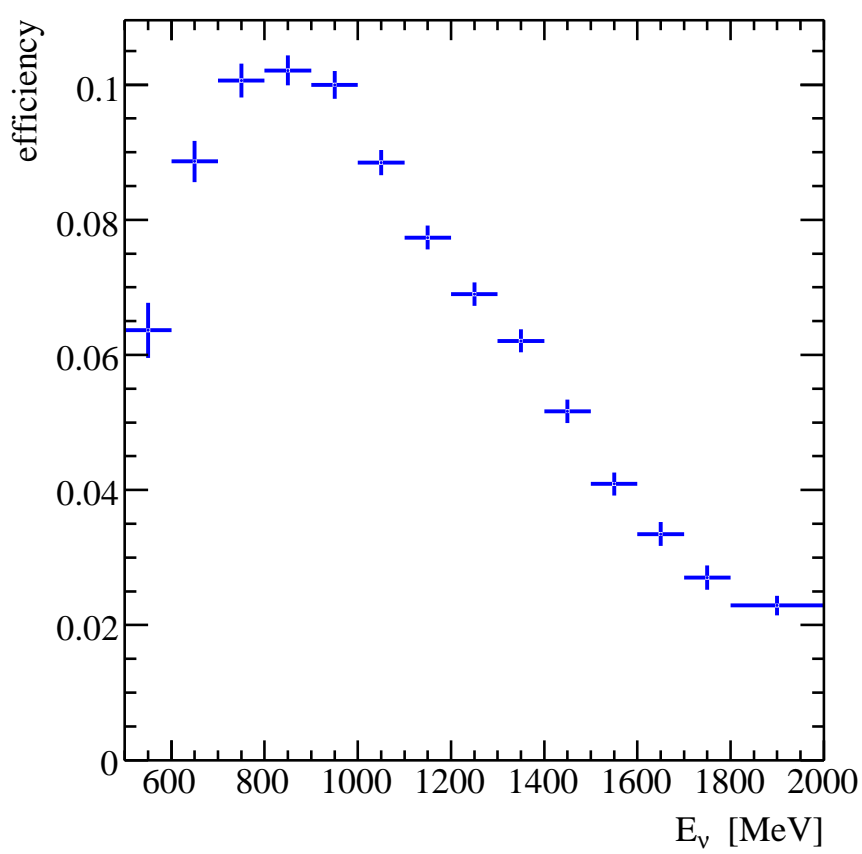

Figure 5.11: The bin efficiency for signal events to pass all cuts as a function of neutrino energy. The errors are statistical on the MC and are appropriately correlated. The total efficiency is $6.4 \%$.

events are more likely to have events that fail to find one of the tracks and subsequently places two tracks on top of each other which causes the reduction in bin efficiency in the first few bins. Additionally, low-energy events sometimes fail the tank hits cut. The constant slope at higher energies is mainly due to events that were lost by having an exiting muon that triggered the veto.

The estimation of the efficiency is the last component needed to calculate the number of interactions given by Eqn. 5.13. The remaining pieces to calculating a crosssection are an estimate of the neutrino flux and the number of target interactions. 


\subsubsection{Neutrino Flux}

An accurate prediction of the neutrino flux is of paramount importance for extracting an absolute cross-section. The flux prediction is discussed in detail in $\S 2.14$. Once the flux has been predicted it is binned the same way as the total cross-section measurement. Fig. 5.12 shows the flux estimate with the binning used for this measurement. The flux-averaged neutrino energy is $\left\langle E_{\nu}\right\rangle_{\Phi}=965 \mathrm{MeV}$.

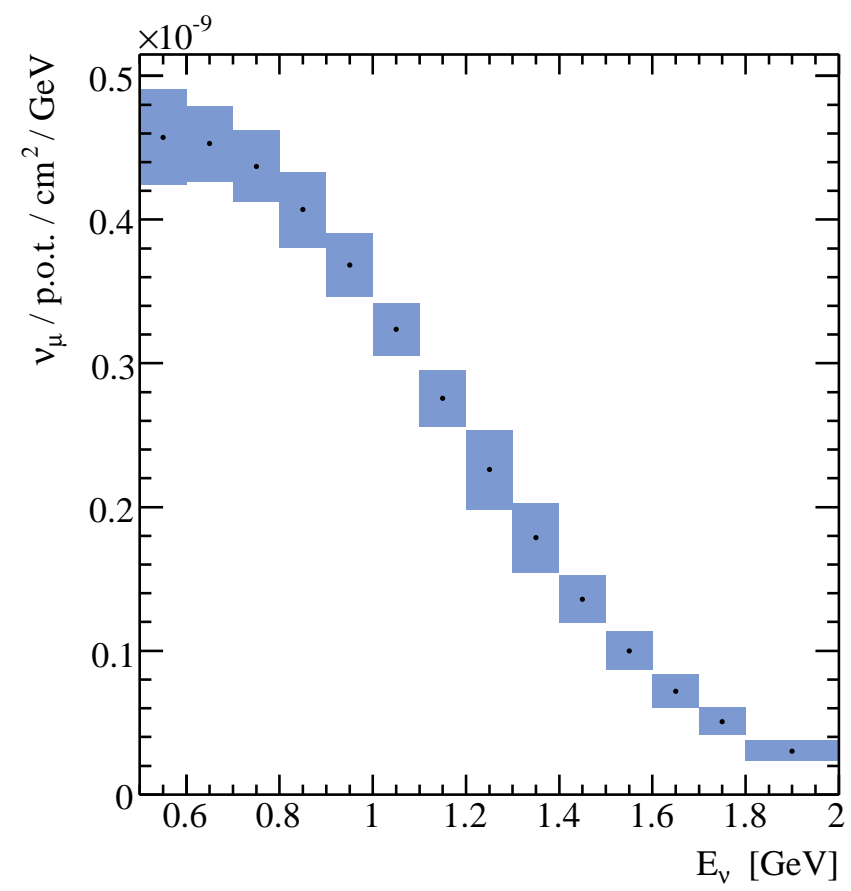

Figure 5.12: The predicted muon neutrino flux as a function of neutrino energy presented in bins and over the range of this analysis. The band shows the total systematic error. The flux-averaged neutrino energy is $\left\langle E_{\nu}\right\rangle_{\Phi}=965 \mathrm{MeV}$.

\subsubsection{Integrated Flux}

The differential cross-section measurements will be restricted to a flux range $E_{\nu} \in$ $(500-2000 \mathrm{MeV})$ to coincide with the same range reported by the total cross-section measurement. The advantages of restricting the flux for the differential measurements 
are three-fold: poorly reconstructed events at low and high neutrino energy are removed, regions of large flux uncertainties are removed, and total flux-averaged cross-sections can be extracted from each measurement and directly compared. The integrated flux is given by

$$
\Phi_{\nu} \equiv \int_{500 \mathrm{MeV}}^{2000 \mathrm{MeV}} \frac{\partial \Phi}{\partial E_{\nu}} d E_{\nu}=(3.545 \pm 0.192) \times 10^{-10} \nu / \mathrm{cm}^{2} / \text { p.o.t. },
$$

where the differential flux presented in Fig. 5.12 has been integrated over.

\subsubsection{Protons on Target}

The flux has been scaled to a single proton interacting with the target. To estimate the actual flux both the differential and integrated fluxes must be multiplied by the number of protons on target in both data and MC. The number of protons predicted in the central value $\mathrm{MC}$ is

$$
n_{\text {p.o.t. }}^{\mathrm{MC}}=4.413 \times 10^{21} \text { p.o.t.. }
$$

In data the measured protons on target are

$$
n_{\text {p.o.t. }}^{\text {Data }}=6.272 \times 10^{20} \text { p.o.t. }
$$

with an uncertainty of around $2 \%$. The estimated total number of neutrinos entering the fiducial volume of the detector is

$$
N_{\nu}=8.71 \times 10^{17} \nu
$$

for $\nu_{\mu}$ neutrinos. For the $10^{6}$ neutrino interactions that occurred within the fiducial volume, a single neutrino had a roughly 1 in $10^{12}$ chance of interacting! Those are rather slim odds.

\subsubsection{Interaction Targets}

The number of interaction targets is calculated within the fiducial volume of the target. The fiducial volume was chosen to encompass a large uniform volume of the 
mineral without including unnecessary materials, such as the PMTs and their support structures. The volume is chosen to be a sphere with a radius of $550 \mathrm{~cm}$ centered in the middle of the tank.

The target is a type of mineral oil composed mainly of carbon and hydrogen. Molecular carbon forms 4 covalent bonds. The carbon and hydrogen atoms in the oil form long chains of carbon with two carbon and two hydrogen atoms filling those bonds. The end of these long chains are occupied by hydrogen atoms so that the ends have a carbon atom bonded to a single carbon atom and three hydrogen atoms. If a chain has $n$ carbon atoms, then the molecular chain can be expressed as $\mathrm{C}_{n} \mathrm{H}_{2 n+2}$. The average length of the hydro-carbon chain is $n \sim 33$ carbon atoms. This implies that the chain can effectively be expressed as the molecule $33\left(\mathrm{CH}_{2.06}\right)$. Expressing the chain this way averages over the two species of the molecules $\mathrm{CH}_{2}$ and $\mathrm{CH}_{3}$. As the relative abundances of the two molecules are all that is important, the length of the chain can be effectively dropped and the interaction target is defined to be a molecule of $\mathrm{CH}_{2.06}$. The molecular weight of hydrogen is $1.00794 \mathrm{~g} / \mathrm{mol}$, and carbon is $12.0107 \mathrm{~g} / \mathrm{mol}$. The density of mineral oil was measured to be $\rho_{\text {oil }}=0.845 \pm 0.001 \mathrm{~g} / \mathrm{cm}^{3}$ [40]. Putting all this together it is a simple matter to calculate the number of interaction targets

$$
N^{\mathrm{targs}}=\frac{4 \pi}{3} r_{\text {fid }}^{3} \cdot \rho_{\text {oil }} \cdot \frac{N_{\text {Avo }}}{\mathrm{CH}_{2.06}}=2.517 \times 10^{31},
$$

where $N_{\mathrm{Avo}}=6.0221415 \times 10^{23} \mathrm{~mol}^{-1}$ is Avogadro's number and $r_{\text {fid }}=550 \mathrm{~cm}$ is the fiducial radius. The cross-section measurements will be presented as measured on $\mathrm{CH}_{2}$ even though the correction is taken into account for the number of targets. This simplifies the use of these cross-sections externally as the details of the molecular structure of the mineral oil are not needed for interpretation of the results. As a nucleonlevel $\mathrm{CC} \pi^{0}$ interaction can only occur on a neutron, it might make sense to multiply the targets by 6 to get the neutron content of the $\mathrm{CH}_{2.06}$. However, as other modes contribute to the observable $\mathrm{CC} \pi^{0}$ cross-section, some events must be on on protons. 
Therefore, the measurement is quoted on $\mathrm{CH}_{2}$.

\subsection{Cross-section Uncertainties}

In order to put the measurements in context, both the statistical and systematic error sources need to be assessed. As the statistical errors are addressed in the next section, the method of systematic uncertainty will be discussed here. Systematic uncertainties are divided into three broad categories: neutrino flux, neutrino cross-sections, and detector sources. The neutrino flux uncertainties come from uncertainties in meson production on the target, both proton and meson reinteractions in the target, and horn modeling. Neutrino cross-section uncertainties primarily affect background contributions, with some dependence of the unfolding on the signal cross-section model. The detector sources are due to the modeling of photon creation and propagation in the mineral oil, meson interactions in the oil, and uncertainties in modeling the detector electronics.

The method for addressing these uncertainties fall into two types: unisims and multisims. The set of unisims are $1 \sigma$ excursions of a parameter that form an error matrix in a distribution by taking the outer product

$$
V_{i j}^{\mathrm{uni}}=\left[\left(\mathbf{x}^{\mathrm{CV}}-\mathbf{x}^{1 \sigma}\right) \otimes\left(\mathbf{x}^{\mathrm{CV}}-\mathbf{x}^{1 \sigma}\right)\right]_{i j}=\left(x_{i}^{\mathrm{CV}}-x_{i}^{1 \sigma}\right)\left(x_{j}^{\mathrm{CV}}-x_{j}^{1 \sigma}\right),
$$

where $\mathbf{x}^{\mathrm{CV}}$ is a distribution, in data, that was inferred by the central value MC. Sometimes referred to as the central value measurement. The distribution $\mathbf{x}^{1 \sigma}$ is a similar measurement; however, the MC used to infer the measurement is from the $1 \sigma$ excursion sample. Unisim errors are typically used when the correlations between parameters are not understood, or thought to be small. Unisims are also used for parameters that require an independent set of $\mathrm{MC}$ to be produced.

The method of multisims takes into account the correlations among the parameters of an underlying distribution, propagating those errors through to the final mea- 
surement. Uncertainties that change the number of measured events can be addressed through reweighting. For instance, the $\pi^{+}$production model is assumed to be a SanfordWang parametrization (see $§ 2.2 .1 .1$ ). This parametrization has 9 parameters, some of which are highly correlated. The fits to external data produce a central value for these parameters along with an error matrix that incorporates the correlations. As the model only predicts the number of neutrinos of a given energy to pass through the detector volume, its effects can be altered through reweighting. However, as the parameters are highly correlated, taking a $1 \sigma$ excursion in each parameter separately is improper. A set of correlated "draws" generate a set of flux histograms that when divided by the central value flux form a reweighting function that can be applied to any event in the central value sample to see how some distribution changes. The beauty of creating a set of correlated draws to apply as a reweighting are that the statistical fluctuations of the sample do not change, and in fact, this method does not introduce any additional statistical error. However, in a situation where the uncertainty does not simply change the number of events, but can change the reconstructed values of the parameters in an event, reweighting is not effective. Additionally, when the source of uncertainty has large correlations between the parameters an extremely large set of MC must be generated to assess these type of errors. The optical model parameters are an example of a set of correlated uncertainties that can change the characteristics of a given event. Regardless of how the multisims are produced, whether through reweighting or multiple MC sets, the covariance matrices are formed the same way. Eqn. 5.26 provides the general idea as to the form of the multisim covariance matrix. If multiple sets of unisims had been created, the covariance matrix would be the average of the set of unisims. Therefore, the covariance matrix of a set of multisim excursions is given by

$$
V_{i j}^{\mathrm{MS}}=\left[\frac{1}{M} \sum_{s=1}^{M}\left(\mathbf{x}^{\mathrm{CV}}-\mathbf{x}^{s}\right) \otimes\left(\mathbf{x}^{\mathrm{CV}}-\mathbf{x}^{s}\right)\right]_{i j}=\frac{1}{M} \sum_{s=1}^{M}\left(x_{i}^{\mathrm{CV}}-x_{i}^{s}\right)\left(x_{j}^{\mathrm{CV}}-x_{j}^{s}\right),
$$

where $\mathbf{x}^{s}$ is the distribution in the $s$ multisim excursion, and $M$ is the total number 
of excursions in that multisim. The total systematic error matrix is sum of all the covariance matrices, whether they be of the unisim or multisim variety.

An error matrix can be separated into three components: a shape error, a normalization error, and a shape/normalization correlation error. This is simply accomplished by taking a central value vector, $\mathbf{x}(n)=\left\{x_{1}, \ldots, x_{n}\right\}$, where $n$ is the number of bins, and transforming to the space, $\mathbf{y}(\mathbf{x})=\left\{x_{1} / N, \ldots, x_{n} / N, N\right\}$, where $N \equiv \sum_{i=1}^{n} x_{i}$ is the integral over $\mathbf{x}$. The covariance of $\mathbf{x}, V(\mathbf{x})$, is then transformed by the Jacobean $\partial \mathbf{y} / \partial \mathbf{x}$ to become the covariance $V(\mathbf{y})$. The elements of $V(\mathbf{y})$ with both $i, j \in(1, \ldots, n)$ is recognized as the shape error matrix. The element $i, j=n+1$ is recognized as the normalization component. The remaining components $i=n+1, j<n+1$ and $i<n+1, j=n+1$ constitute the shape/normalization correlation. However, the matrix $V(\mathbf{y})$ needs to be transformed back into $\mathbf{x}$ while keeping careful track of each component. Doing so gives

$$
\begin{aligned}
V_{i j}(\mathbf{x}) & =\left(V_{i j}-\frac{x_{i}}{N} \sum_{k} V_{k j}-\frac{x_{j}}{N} \sum_{k} V_{i k}+\frac{x_{i} x_{j}}{N^{2}} \sum_{k l} V_{k l}\right) \\
& +\left(\frac{x_{i}}{N} \sum_{k} V_{k j}-2 \frac{x_{i} x_{j}}{N^{2}} \sum_{k l} V_{k l}+\frac{x_{j}}{N} \sum_{k} V_{i k}\right) \\
& +\frac{x_{i} x_{j}}{N^{2}} \sum_{k l} V_{k l} \\
& =V_{i j},
\end{aligned}
$$

where the first row is the shape error, the second is the shape/normalization error, and the third row is the normalization error. This allows for the error in a bin to be broken up by the types of error explicitly. The total normalization uncertainty is the square root of the sum of the error matrix, $\sqrt{\sum_{i j} V_{i j}}$.

To measure the correlation between bins of the measurement the correlation matrix is defined

$$
\rho_{i j} \equiv \frac{V_{i j}}{\sqrt{V_{i i} V_{j j}}},
$$

whose values are between -1 and 1 . The value of 1 implies full correlation between bins, 
0 implies no correlation, and -1 implies full anti-correlation.

\subsubsection{Statistical Errors}

Statistical errors are assessed in both data and MC and applied to the measurements. The statistical error comes from each component of the inferred number of events (Eqn. 5.13), reproduced here,

$$
N_{i}^{\prime}=\frac{\sum_{j} U_{i j}\left(N_{j}-B_{j}\right)}{\epsilon_{i}}
$$

where $N_{j}$ is a bin of data, $B_{j}$ is a bin of background, $U_{i j}$ is an unfolding matrix element, and $\epsilon_{i}$ is the bin efficiency. The statistical covariance matrix of the vector $\mathbf{N}^{\prime}$ is defined as

$$
\mathbf{V}\left(\mathbf{N}^{\prime}\right)=\frac{\partial \mathbf{N}^{\prime}}{\partial \mathbf{U}} \mathbf{V}(\mathbf{U}) \frac{\partial \mathbf{N}^{\prime T}}{\partial \mathbf{U}}+\frac{\partial \mathbf{N}^{\prime}}{\partial \epsilon} \mathbf{V}(\epsilon) \frac{\partial \mathbf{N}^{\prime T}}{\partial \epsilon}+\frac{\partial \mathbf{N}^{\prime}}{\partial \mathbf{N}} \mathbf{V}(\mathbf{N}) \frac{\partial \mathbf{N}^{\prime T}}{\partial \mathbf{N}}+\frac{\partial \mathbf{N}^{\prime}}{\partial \mathbf{B}} \mathbf{V}(\mathbf{B}) \frac{\partial \mathbf{N}^{\prime T}}{\partial \mathbf{B}}
$$

where each covariance matrix on the right hand side is diagonal. Each of these sources of error are assumed to follow Poisson statistics. However, some of these quantities are scaled by prefactors, and others have internal correlations that need to be accounted for.

The statistical error on the data should technically come from the MC prediction for the number of data events. There is no uncertainty on the number of measured events in data. The uncertainty comes from repeating the experiment with the same systematics. As this relies on an accurate signal MC, in this case it is impractical. Additionally, as the MC underpredicts the data by over $20 \%$, the statistical uncertainty would be smaller than it should be. A more accurate model would have predicted the number of data events in a given bin to within the statistical error. To that end, the number of events in a data bin is used as an estimator of the statistical error even though fluctuations in the data can cause the statistical error to over(under)predict depending 
on direction of the fluctuation. The covariance matrix for data is

$$
V_{i j}(\mathbf{N})=N_{i} \delta_{i j}
$$

where $\delta_{i j}$ is the Kronecker delta function, and $N_{i}$ is the number of events in bin $i$ of data distribution.

The background distribution is scaled to match the proton on target normalization of the data. Defining $S_{\text {p.o.t. }} \equiv n_{\text {p.o.t. }}^{\text {Data }} / n_{\text {p.o.t. }}^{\mathrm{MC}} \simeq 0.15$ the covariance matrix is

$$
V_{i j}(\mathbf{B})=S_{\text {p.o.t. }}^{2} B_{i} \delta_{i j}
$$

The unfolding statistical covariance matrix is derived in $\S \mathrm{A} .2$ and is given by Eqn. A.14. The unfolding covariance is derived by propagating the data statistics along with the MC statistics of the unfolding matrix. Plugging the data and background covariances into Eqn. A.14 the unfolded background subtracted data covariance matrix is

$$
V_{i j}[\mathbf{U}(\mathbf{D}-\mathbf{B})]=\sum_{k}^{\text {bins }}\left\{U_{i k}\left(N_{k}+S_{\text {p.o.t. }}^{2} B_{k}\right) U_{j k}+\frac{U_{i k}^{2}}{R_{i k}}\left(N_{k}-S_{\text {p.o.t. }} B_{k}\right)^{2}\left(\delta_{i j}-U_{j k}\right)\right\},
$$

where $R_{i k}$ is an element of the bin migration matrix. This matrix is symmetric, but no longer completely diagonal. Unfolding has the effect of reducing and smoothing the diagonal errors at the sacrifice of introducing correlations into the statistical errors.

The bin efficiency is given by $\epsilon_{i}=x_{i} / n_{i}$ where $x_{i}$ is the number of events passing cuts in bin $i$, and $n_{i}$ is total number of events in the fiducial volume prior to cuts. As $x$ is a subset of $n$, the correlations must be taken into account. Properly accounting for these correlations gives,

$$
V_{i j}(\epsilon)=\frac{\epsilon_{i}\left(1-\epsilon_{i}\right)}{n_{i}} \delta_{i j}
$$

which is a diagonal covariance matrix.

Taking the appropriate derivatives, the statistical covariance matrix is,

$$
V_{i j}\left(\mathbf{N}^{\prime}\right)=\frac{1}{\epsilon_{i} \epsilon_{j}} V_{i j}[\mathbf{U}(\mathbf{N}-\mathbf{B})]+\frac{N_{i}^{\prime} N_{j}^{\prime}}{\epsilon_{i} \epsilon_{j}} V_{i j}(\epsilon)
$$


which is mostly diagonal with slight off-diagonal correlations. This method of calculating the statistical error can be generalized to any number of dimensions. As the background subtraction and unfolding for the differential cross-sections were performed on two dimensional distributions, the statistical error was calculated for each bin in the distribution then combined for the one dimensional results.

\subsubsection{Flux Sources}

The neutrino flux is created by the decays of mesons on the secondary beamline. The uncertainties come from a few sources:

- The $\pi^{+}$inclusive cross-section, $\sigma\left(p+B e \rightarrow \pi^{+}+X\right)$.

- The $\pi^{-}$inclusive cross-section, $\sigma\left(p+B e \rightarrow \pi^{-}+X\right)$.

- The $K^{+}$inclusive cross-section, $\sigma\left(p+B e \rightarrow K^{+}+X\right)$.

- The $K^{0}$ inclusive cross-section, $\sigma\left(p+B e \rightarrow K^{0}+X\right)$.

- Proton and meson reinteractions in the target, horn, and shielding.

- Horn current and current skin depth.

For this measurement, of the proton-beryllium inclusive cross-sections the $\pi^{+}$uncertainties dominate. The last two flux sources are determined by a set of unisims that produce an error matrix on the flux. This error matrix is used by the reweighting and is collectively referred to as "beam unisims" even though the final error matrix was gotten through the method of multisimming. Fig. 5.13 shows the relative flux uncertainties for the two dominant flux uncertainty sources, $\pi^{+}$production and beam unisims, and for a source that dominates at energy above $3 \mathrm{GeV}$, the beam $K^{+}$uncertainty. Fig. 5.14 shows the correlation matrix associated with the $\pi^{+}$production uncertainty. This matrix shows strong correlations between neighboring bins, and some fairly strong 


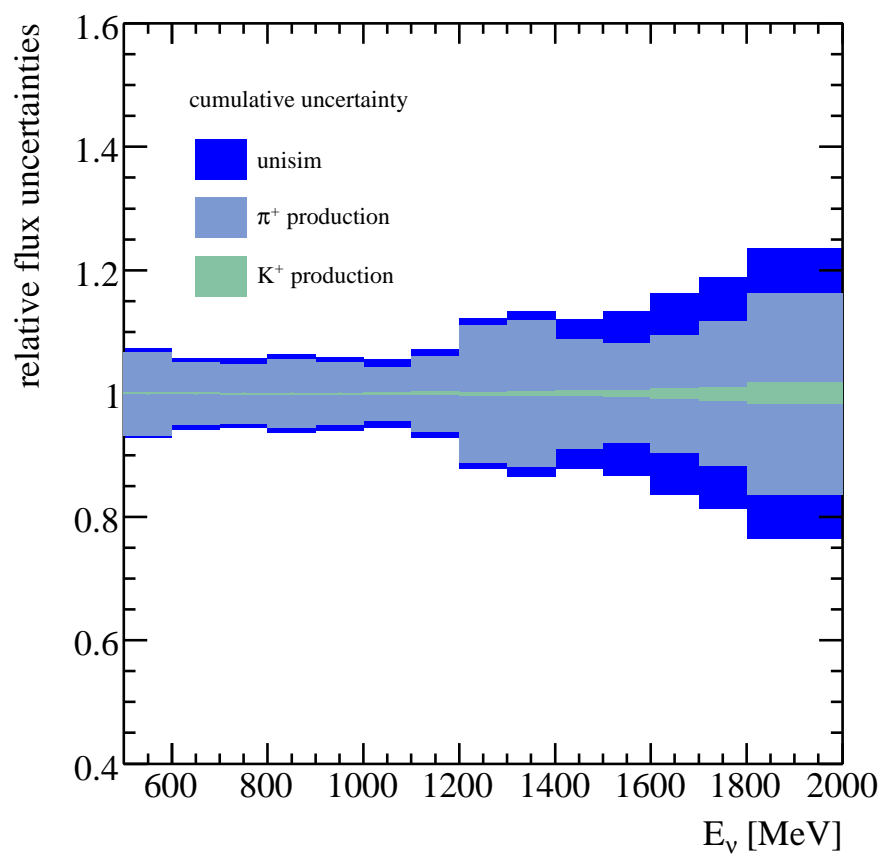

Figure 5.13: Relative flux uncertainties for $\pi^{+}$production, $K^{+}$production, and the set of beam unisims. The $K^{+}$errors dominate at energies above this range, and the $\pi^{+}$ errors get really large below $400 \mathrm{MeV}$. The error matrices are added in succession, with the beam unisim errors representing the total error.

anti-correlations up to the $50 \%$ level. The correlations are a result of the underlying correlations between the Sanford-Wang production cross-sections. Table 5.3 gives the relative error for the integrated flux.

\subsubsection{Detector Sources}

The sources of uncertainty associated with the detector come from the electronics, the optical model, and cross-section models. Out of these uncertainties, only the crosssection model can be addressed via reweighting. 


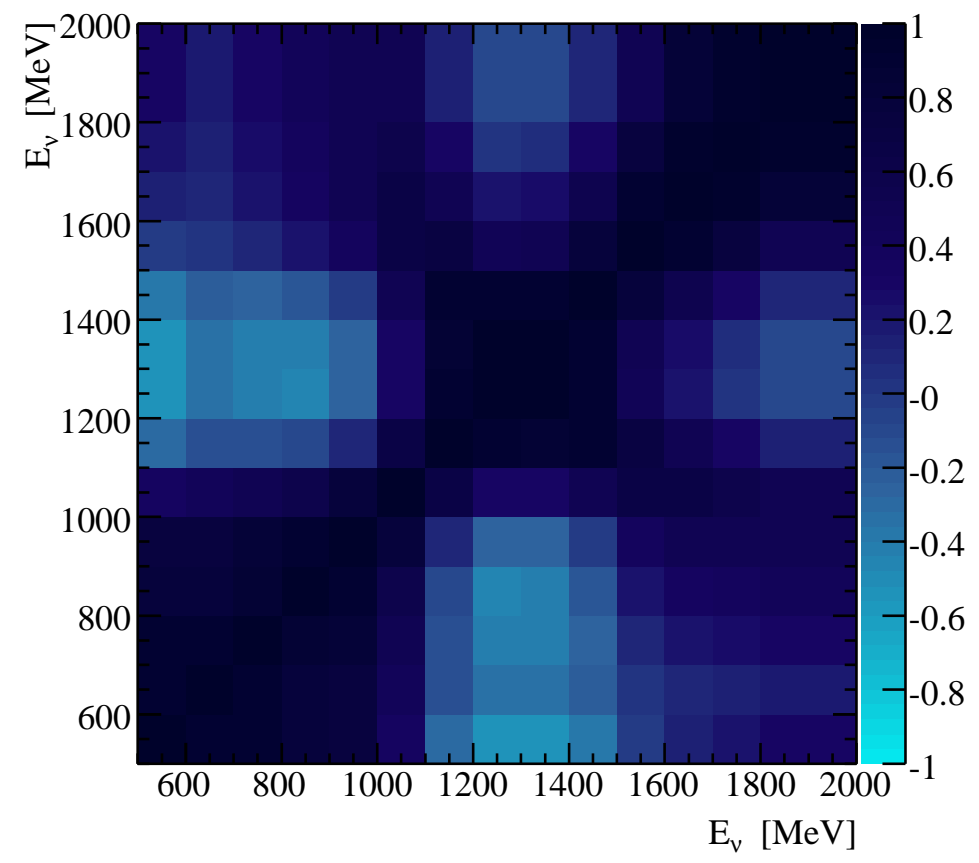

Figure 5.14: The correlation matrix for $\pi^{+}$production defined by Eqn. 5.32. While some bins are highly correlated, the maximum anti-correlation is around $50 \%$.

\subsubsection{Discriminator Threshold}

The discriminators are set to reject noise and only fire if the PMT pulse is above a threshold. The thresholds are set at 0.1 photo-electron (pe). The uncertainty comes from different amounts of noise associated with each PMT, possibly different baselines, and the effect of the threshold on determining the time of the hit. The time of the hit is determined when the PMT signal first crosses the threshold. As described in $§ 2.1 .4 .2$ the shapes of the PMT pulses differ depending on the amount of charge associated with the event. Larger pulses tend to cross threshold faster than smaller ones. Changing the threshold then causes two effects; the hit time changes, and smaller pulses might be lost. To estimate the effect of changing the threshold, the discriminator threshold was changed to 0.2 pe in an independent MC sample. The level was chosen because 0.3 pe seemed too large, so the conservative estimate was to split the difference [95]. 
Table 5.3: The normalization uncertainties for all flux uncertainties. These uncertainties represent the uncertainties on the integrated flux.

\begin{tabular}{l|c} 
Flux error source & Normalization uncertainty \\
\hline \hline Beam unisims & $3.8 \%$ \\
$\pi^{+}$production & $3.8 \%$ \\
$K^{+}$production & $0.2 \%$ \\
$\pi^{-}$production & $0.01 \%$ \\
$K^{0}$ production & $0.01 \%$ \\
\hline Total & $5.4 \%$
\end{tabular}

\subsubsection{QT Correlation}

The "charge/time" correlation is due to larger PMT pulses crossing threshold sooner than smaller pulses. This is similar to the discriminator threshold effect, except instead of varying the threshold, the position of the pulse is adjusted. The relationship between the start time of a pulse as a function of total charge is modeled in the MC, though tuned in the data using the calibration laser (see §2.1.4.4). For a given subevent, there will be tubes with small charges at the beginning and end of the time window. The goal of this correction is to adjust the timing position of these low charge PMT hits such that the prompt peak is unaffected while mocking up the charge-time distributions seen in data. As the MC predicts more low charge PMTs in an event, this unisim estimates the uncertainty by turning off the low charge events [95].

\subsubsection{Optical Model}

The optical model, which was discussed in $\S 2.1 .4 .1$, has 35 adjustable parameters that have been tuned by external measurements and calibration data. Many of these parameters are correlated, implying that the method of multisimming will be required to get an accurate estimation of the optical model uncertainty. As the optical model changes the properties of an event, reweighting is also not appropriate. The optical model must be multisimmed through an enormous set of MC that take correlated draws 
Table 5.4: Parameters used in the optical model variations. Table reproduced from Ref. [96]

\begin{tabular}{lc|lc} 
Property & \# pars. & Property & \# pars. \\
\hline \hline Cerenkov scale factor & 1 & index of refraction & 3 \\
fluor scintillation yield & 4 & extinction length & 5 \\
fluor fluorescence yield & 4 & scattering & 3 \\
fluor UV fluorescence yield & 4 & reflections & 2 \\
fluor time constant & 4 & PMT angular efficiency & 2 \\
Birk's law coefficients & 2 & old/new PMT scale factor & 1
\end{tabular}

of the 35 parameters and generate a new set of MC. The problem is that to fully explore the space that the 35 parameters can occupy, would take probably thousands of draws. However, this is completely impractical given the cpu time needed to generate such a set of $\mathrm{MC}$, the disk space required to store such a set, and the analysis time of this set. The goal is generate a "good enough" set of these excursions. A set of 67 data-sized MC were generated to explore the space. Unfortunately, this was as large as a reasonable set of MC that could be generated. However, as long as the multisims sample the space, which the 67 optical model multisims do, then this set of multisims are adequate.

Light propagation in the mineral oil is affected by several known phenomena: Elastic scattering (Rayleigh), scintillation and fluorescence (Raman and Stoke's effect), extinction, reflections, and PMT efficiencies. The optical model parameters are given in Table 5.4. The base optical model uses only the primary fluor and ignores the effect due to the other 3 known fluors. However, the 3 additional fluors are included for variations in the optical model. As the base simulation does not keep track of photons with wavelengths below $250 \mathrm{~nm}$ (UV) because they can not be seen by the PMTs, the fluorescence of these photons can produce photons with longer wavelengths and are included in the optical model variations. Additionally, the second parameter of Birk's law (Eqn. 2.28), $B_{2}$, is included for variations. 


\subsubsection{Cross-sections}

The NUANCE neutrino generator [57] predicts neutrino interactions over an energy range of $100 \mathrm{MeV}$ to $1 \mathrm{TeV}$. The set of interactions includes 99 exclusive channels that contribute to the total cross-section. These exclusive interactions were tuned to existing data sets; however, at MiniBooNE energies, many of the measurements are either inadequate or non-existent. These exclusive interactions are predicted at the nucleon level. Additionally, NUANCE incorporates a final state interaction (FSI) model of Smith and Moniz [64] to predict the interactions of the nucleon-level final state with the nuclear medium. This model assumes a relativistic Fermi gas with a momentum cutoff at $220 \pm 30 \mathrm{MeV} / c$, and a $34 \pm 9 \mathrm{MeV}$ binding energy. As the $\mathrm{CC} \pi^{0}$ crosssection measurement is of observable $\mathrm{CC} \pi^{0}$, the details of the FSI model are not so important. Many nucleon-level interactions make up each observable mode; variations of those modes effect the overall rate of observable interactions. The common feature to the dominant backgrounds $\mathrm{CCQE}, \mathrm{CC} \pi^{+}$, and $\mathrm{CCmulti}-\pi$ is the axial vector dipole form. Each mode uses a different axial mass, and they are varied within their measured uncertainties.

Ideally, the cross-section uncertainties would only affect the background subtraction. However, as the unfolding matrix requires a Bayesian prior for the signal, a portion of the cross-section uncertainty is due to the unfolding.

\subsubsection{Charge exchange and Absorption}

The single largest uncertainty to these measurements come from $\pi^{+} \rightarrow \pi^{0}$ and $\pi^{+} \rightarrow \hbar$ in the mineral oil, referred to as charge exchange and absorption respectively. This is such a large uncertainty because $\mathrm{CC} \pi^{+}$events are a much larger sample than $\mathrm{CC} \pi^{0}$ and every $\mathrm{CC} \pi^{+}$event that ends up in the $\mathrm{CC} \pi^{0}$ sample "lost" a $\pi^{+}$somehow, and the uncertainties on these processes in the mineral oil are extremely large. This 
process is distinguished from charge exchange and absorption in the target nucleus, as those are considered FSI effects. Fig. 5.15 shows the exclusive cross-sections for $\pi^{+} \rightarrow \pi^{0}$
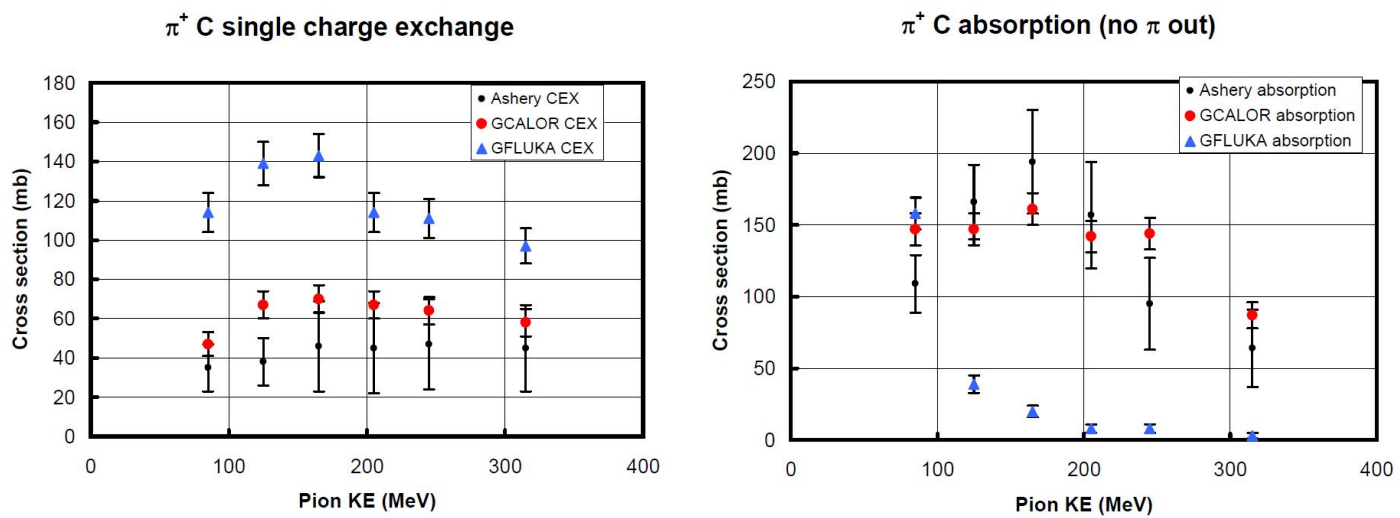

Figure 5.15: The cross-sections for $\pi^{+} \rightarrow \pi^{0}$ (left) and $\pi^{+} \rightarrow \lambda$ (right) off carbon as a function of $\pi^{+}$kinetic energy. Data are from Ref. [97]. The hadronic models, GFLUKA [98] and GCALOR [99], seem to prefer GCALOR to match the data. The uncertainties are set by the data.

and $\pi^{+} \rightarrow \lambda$ off carbon. The GCALOR [99] hadronic model is used with errors set by the Ashery experiment data [97]. The uncertainties are set by the data and are $50 \%$ and $35 \%$ respectively.

\subsubsection{Systematic Error Discussion}

Many sources of systematic error were considered during this measurement. The previously measured MiniBooNE cross-section measurements were dominated by beam related uncertainties. Here, in fact, they are the second largest sources of uncertainty; the neutrino interaction cross-sections are third. At low neutrino energies the discriminator threshold uncertainty dominates. However, the largest source of uncertainty, $\pi^{+}$ charge exchange and absorption, dominates because of the much larger $\mathrm{CC} \pi^{+}$sample. The relative production of observable to $\mathrm{CC} \pi^{+}$to $\mathrm{CC} \pi^{0}$ is roughly a factor of 12. A $10 \%$ total charge exchange and absorption probability would contribute a $\mathrm{CC} \pi^{+}$ background roughly equal to the signal. Add $50 \%$ errors on that probability, and it is 
easy to see how this could affect this measurement. Since there is only about a $25 \%$ contamination in the $\mathrm{CC} \pi^{0}$ sample from observable $\mathrm{CC} \pi^{+}$, a $50 \%$ uncertainty on that background yields a $12.5 \%$ uncertainty on the extracted signal. Thankfully, the level of observable $\mathrm{CC} \pi^{+}$was previously measured [71], which ties the level of observable $\mathrm{CC} \pi^{+}$ to the MiniBooNE data set reducing the $\mathrm{CC} \pi^{+}$production uncertainty to well below the NUANCE prediction.

Additionally, two sources of uncertainty have been ignored. The uncertainty in the number of protons on target is at the $2 \%$ level. The second error that has been ignored is the uncertainty on the density of the mineral oil. This is much smaller than $2 \%$ and is justifiably ignored.

\section{5 $\quad \mathrm{CC} \pi^{0}$ Total Cross-section}

Fig. 5.16 shows the total observable $\mathrm{CC} \pi^{0}$ cross-section as function of neutrino energy. The data shows an enhancement over prediction in every bin of the measurement. Previous measurements were on deuterium $[85,86,87]$ and also showed an enhancement compared with the Rein-Seghal model [62]. This is similar to what has been observed in other modes. The total systematic error is $18.7 \%$, and the total statistical error is $3.3 \%$. Flux averaging over the total cross-section gives $\langle\sigma\rangle_{\Phi}=(9.05 \pm 1.44) \times 10^{-39} \mathrm{~cm}^{2} / \mathrm{CH}_{2}$ with the caveat that the flux errors were not accounted for properly on the background subtraction. However, the fluctuations on the background are anti-correlated with the flux factor implying that the true error is smaller than this error, though not by any significant margin. Table 5.5 summarizes the normalization errors on the observable $\mathrm{CC} \pi^{0}$ total cross-section measurement. Table B.1, in the appendix, gives the numerical values for the total cross-section, total systematic error, statistical error, and correlation matrix. 
Table 5.5: Normalization uncertainties on the observable $\mathrm{CC} \pi^{0}$ total cross-section measurement.

\begin{tabular}{l|r}
\multicolumn{1}{c|}{ Source } & Uncertainty \\
\hline \hline Statistical & $3.27 \%$ \\
\hline$\pi^{+} \rightarrow \pi^{0}$ and $\pi^{+} \rightarrow \lambda^{+}$ & $12.88 \%$ \\
Beam Unisims & $7.53 \%$ \\
Beam $\pi^{+}$ & $7.28 \%$ \\
Cross-sections & $5.78 \%$ \\
Discriminator & $5.66 \%$ \\
Optical Model & $2.82 \%$ \\
QT correlation & $1.05 \%$ \\
Beam $K^{+}$ & $0.91 \%$ \\
CC $\pi^{+}$cross-section & $0.52 \%$ \\
Beam $\pi^{-}$ & $0.29 \%$ \\
Hadronic & $0.21 \%$ \\
Beam $K^{0}$ & $0.03 \%$ \\
\hline Total Normalization & $18.74 \%$
\end{tabular}

\section{6 $\quad \mathrm{CC} \pi^{0}$ Differential Cross-sections}

The differential cross-sections as a function of final state kinematics presented here are flux averaged, but not over the entire flux. This is because the flux uncertainties get rather large below threshold, and the reconstruction quality gets worse at higher neutrino energy. It is not possible to explicitly restrict the flux on an event-by-event basis. For these measurements the neutrino energy is measured. Therefore, each of the distributions can be presented as a function of neutrino energy. This allows for an unfolding of the background subtracted rate in dimensions of neutrino energy and the final state variable. Neutrinos that reconstruct outside of the chosen range in neutrino energy are cut from the sample. The range of neutrino energy has been restricted to the reported range of the total cross-section measurement, or $E_{\nu} \in(500-2000 \mathrm{MeV})$. The rate is then collapsed in the dimension of neutrino energy, leaving a 1-D differential cross-section in terms of the chosen final state variable. Expressing the differential crosssections as a function of neutrino energy has large statistical errors in each bin and is 
therefore not reported. The flux-restricted differential cross-section measurements allow for direct comparisons to the total cross-section measurement in Fig. 5.16. For each measurement the total flux-averaged cross-section is measured and directly compared. Differences in these values are strictly due to bin efficiency averaging, as each variable will have a different efficiency function.

\subsection{1 $\quad Q^{2}$ Cross-section}

Fig. 5.17 shows the flux-averaged observable $\mathrm{CC} \pi^{0}$ differential in $Q^{2}$. This crosssection has only previously been measured inclusively with other single pion modes $[85$, 86, 87]. The measured cross-section shows a low $Q^{2}$ suppression and overall harder spectrum. The total systematic error is $16.1 \%$. Integrating over this cross-section gives $\langle\sigma\rangle_{\Phi}=(9.28 \pm 1.50) \times 10^{-39} \mathrm{~cm}^{2} / \mathrm{CH}_{2}$.

\subsubsection{Muon Kinematic Cross-sections}

Fig. 5.18 shows the flux-averaged observable $\mathrm{CC} \pi^{0}$ differential in $E_{\mu}$. This crosssection has never been measured before, and shows an enhancement with respect to prediction. The total systematic error is $15.8 \%$. The flux-averaged total cross-section is $\langle\sigma\rangle_{\Phi}=(9.20 \pm 1.47) \times 10^{-39} \mathrm{~cm}^{2} / \mathrm{CH}_{2}$.

Fig. 5.19 shows the flux-averaged observable $\mathrm{CC} \pi^{0}$ differential in $\cos \theta_{\mu}$. This cross-section has never been measured before, and shows an enhancement with respect to prediction everywhere but the most forward direction. This suppression in the forward direction is similarly seen in the CCQE but not in the observable $\mathrm{CC} \pi^{+}$. As CCQE and $\mathrm{CC} \pi^{0}$ have no coherent contribution, this suppression is expected. The total systematic error is $17.4 \%$. The flux-averaged total cross-section is $\langle\sigma\rangle_{\Phi}=$ $(9.10 \pm 1.50) \times 10^{-39} \mathrm{~cm}^{2} / \mathrm{CH}_{2}$. 


\subsubsection{Pion Kinematic Cross-sections}

Fig. 5.20 shows the flux-averaged observable $\mathrm{CC} \pi^{0}$ differential in $\left|\mathbf{p}_{\pi^{0}}\right|$. This cross-section has never been measured before and shows a large enhancement at low momentum, and agreement at high momentum. This implies lower momentum $\pi^{0}$ than are expected by the relativistic Fermi gas (RFG) model from a $\Delta$ decay. Qualitatively, either the $\Delta$ undergoes more scattering in the nucleus than is expected, or a $\pi^{0}$ is created through some scattering process (e.g. $\pi^{+} \rightarrow \pi^{0}$ ) that is not modeled correctly by the RFG model. The total systematic error is $15.9 \%$. The flux-averaged total cross-section is $\langle\sigma\rangle_{\Phi}=(9.03 \pm 1.54) \times 10^{-39} \mathrm{~cm}^{2} / \mathrm{CH}_{2}$.

Fig. 5.20 shows the flux-averaged observable $\mathrm{CC} \pi^{0}$ differential in $\cos \theta_{\pi^{0}}$. This cross-section has never been measured before, and shows greater enhancements in the forward and backward directions than are predicted. This is similar to what was observed in the observable $\mathrm{NC} \pi^{0}$ measurement [75]. The total systematic error is $16.3 \%$. The flux averaged total cross-section is $\langle\sigma\rangle_{\Phi}=(9.54 \pm 1.55) \times 10^{-39} \mathrm{~cm}^{2} / \mathrm{CH}_{2}$.

\subsubsection{Flux-Averaged Total Cross-section}

Each of the cross-section measurements provide a flux-averaged total cross-section. The largest and smallest reported values vary by $5.6 \%$, well within the $16-19 \%$ range of reported errors on the flux-averaged cross-section measurements. The differences are attributed to biases introduced through the bin efficiencies for the choice of binning in each measurement. As there is no a priori reason to choose one measurement over another, the measurements are averaged assuming 100\% correlated errors. The average and covariance are given by

$$
\begin{aligned}
\langle\bar{\sigma}\rangle_{\Phi} & =\frac{1}{n} \sum_{i}\langle\sigma\rangle_{\Phi, i} \\
V\left(\langle\bar{\sigma}\rangle_{\Phi}\right) & =\left(\frac{1}{n} \sum_{i} \sqrt{V\left(\langle\sigma\rangle_{\Phi, i}\right)}\right)^{2}
\end{aligned}
$$


where $\langle\sigma\rangle_{\Phi, i}$ is the $i^{\text {th }}$ measurement, $n$ is the number of measurements, and $V(x)$ is the covariance of $x$. The average of the flux-averaged total cross-sections is $\langle\bar{\sigma}\rangle_{\Phi}=$ $\left(9.20 \pm 1.50_{\text {syst. }} \pm 0.3_{\text {stat. }}\right) \times 10^{-39} \mathrm{~cm}^{2} / \mathrm{CH}_{2}$ for a flux-averaged neutrino energy of 965 $\mathrm{MeV}$. The results are summarized in Table 5.6.

A simple average is chosen over the simple weighted average and also the Lyons method for combining correlated measurements [100]. The simple weighted average weights each measurement by the uncertainty, however, it does not correctly account for correlations and the uncertainty on the weighted average would have been artificially small. As for the Lyons method, also a weighted average, the problem was that the systematic uncertainties are thought to be almost, if not fully, correlated. The systematic covariance matrix formed between the measurements is then non-invertible; invertibility is a requirement for the Lyons method to work. The authors of Ref. [100] suggest adding small terms to the diagonal elements to break the correlations, however, it is not clear what those "small" terms should have been. Regardless, the simple average is thought to be adequate as the measurements agree well within their uncertainties, and the simple average ensures the uncertainty on the average is not artificially reduced.

Table 5.6: Summary of the flux-averaged total cross-sections calculated from each crosssection measurement. The average cross-section is calculated assuming $100 \%$ correlated systematics. The flux-averaged neutrino energy is $\left\langle E_{\nu}\right\rangle_{\Phi}=965 \mathrm{MeV}$.

\begin{tabular}{l|c} 
cross-section & $\langle\sigma\rangle_{\Phi}\left[\times 10^{-39} \mathrm{~cm}^{2}\right]$ \\
\hline \hline$\sigma\left(E_{\nu}\right)$ & $9.05 \pm 1.44$ \\
$\partial \sigma / \partial Q^{2}$ & $9.28 \pm 1.50$ \\
$\partial \sigma / \partial E_{\mu}$ & $9.20 \pm 1.47$ \\
$\partial \sigma / \partial \cos \theta_{\mu}$ & $9.10 \pm 1.50$ \\
$\partial \sigma / \partial\left|\mathbf{p}_{\pi^{0}}\right|$ & $9.03 \pm 1.54$ \\
$\partial \sigma / \partial \cos \theta_{\pi^{0}}$ & $9.54 \pm 1.55$ \\
\hline \multicolumn{1}{c}{$\langle\bar{\sigma}\rangle_{\Phi}$} & $9.20 \pm 1.50$
\end{tabular}



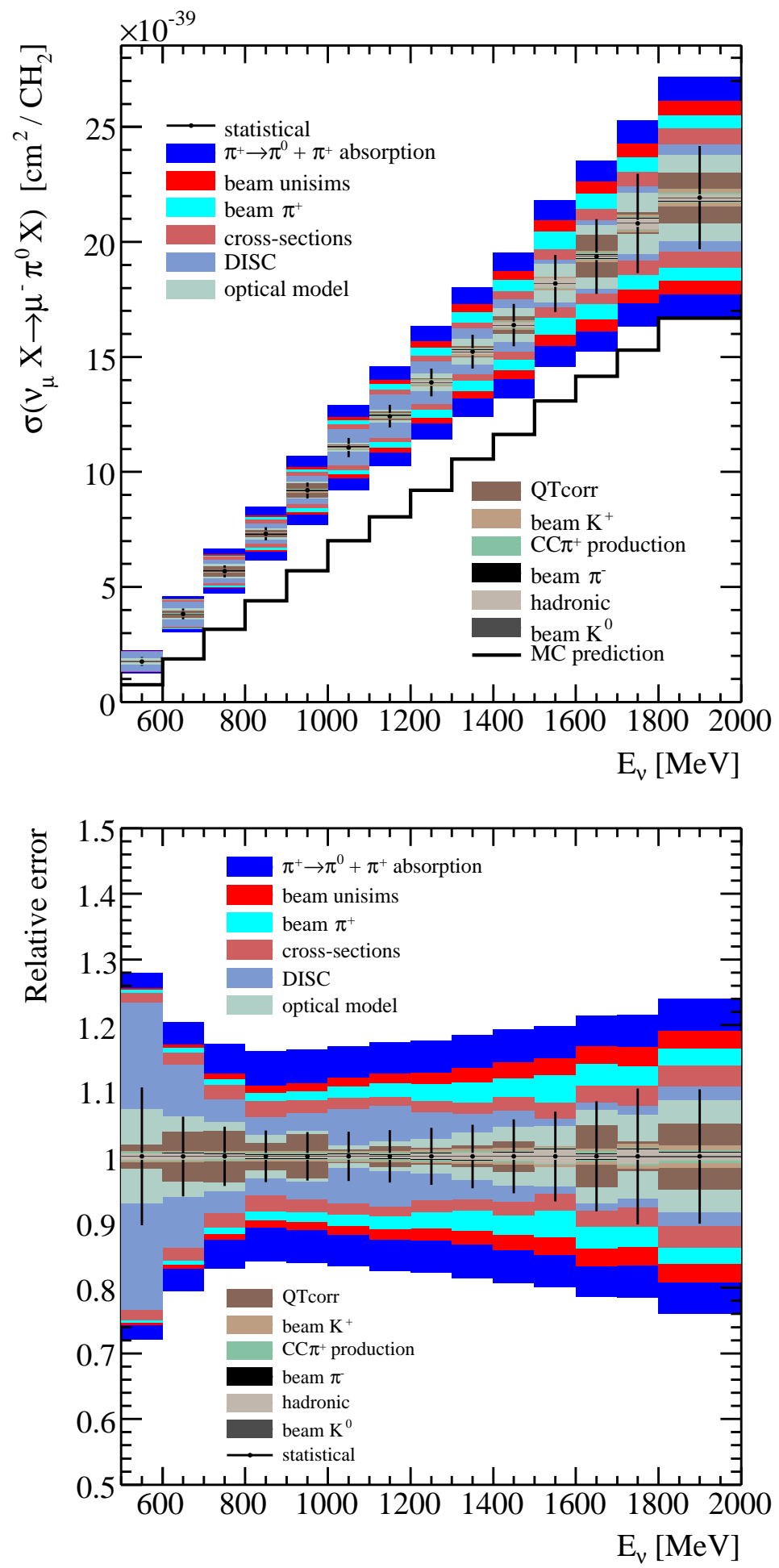

Figure 5.16: Top: The total observable $\mathrm{CC} \pi^{0}$ cross-section as a function of neutrino energy. Each error band is plotted in a stacked fashion where all error matrices with a smaller normalization error is added to labeled error band. Beam $\mathrm{K}^{0}$ production has the smallest normalization component, and $\pi^{+} \rightarrow \pi^{0}$ and $\pi^{+}$absorption is the largest error and represents the total systematic error. The flux-averaged cross-section over the range $E_{\nu} \in(500-2000 \mathrm{MeV})$ is $\langle\sigma\rangle_{\Phi}=(9.05 \pm 1.44) \times 10^{-39} \mathrm{~cm}^{2} / \mathrm{CH}_{2}$. Bottom: The relative errors found by dividing out the measured cross-section. The total systematic normalization error is $18.7 \%$. 

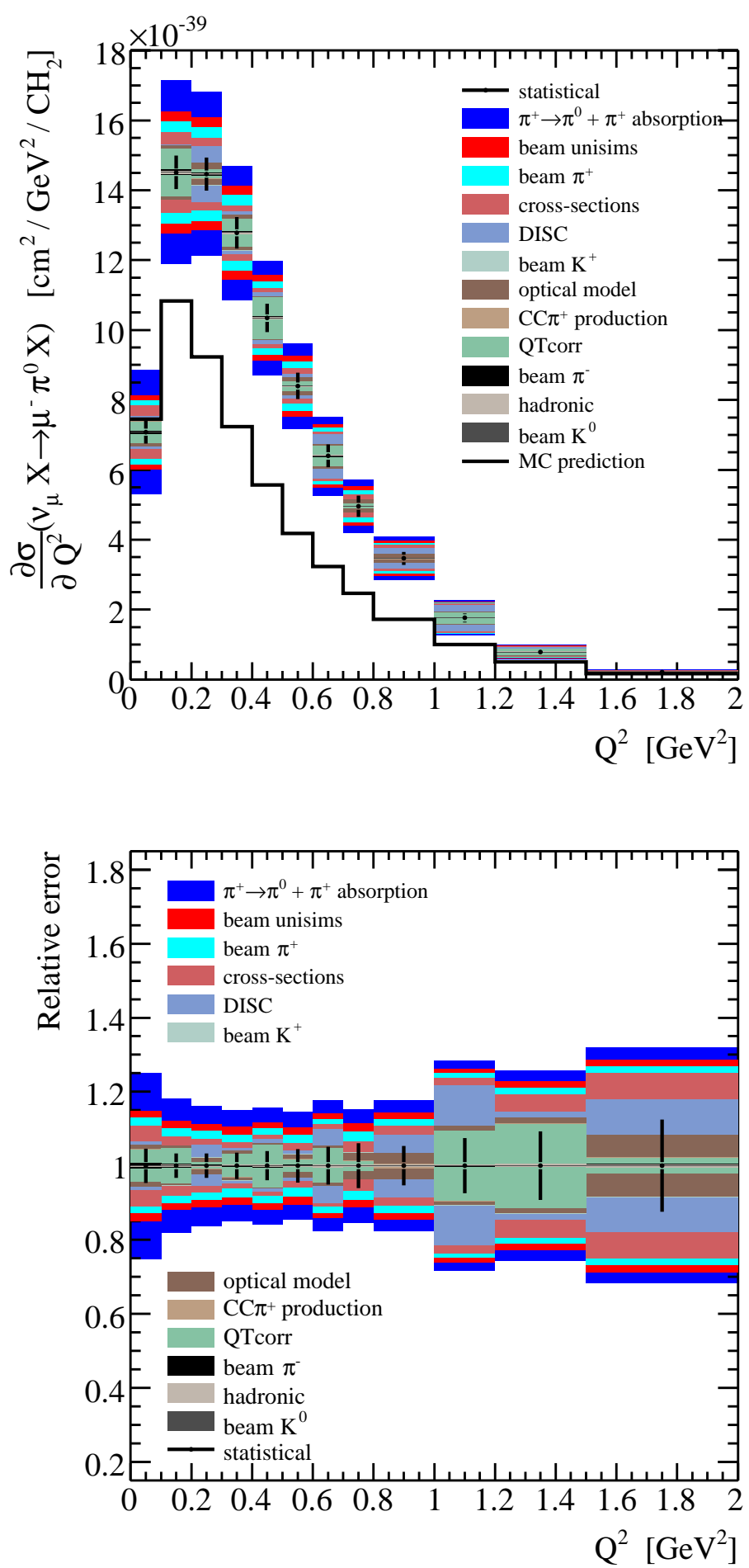

Figure 5.17: Top: The flux-averaged observable $\mathrm{CC} \pi^{0}$ cross-section differential in $Q^{2}$ over the flux range $E_{\nu} \in(500-2000 \mathrm{MeV})$. The total flux-averaged cross-section calculated by integrating over $Q^{2}$ is $\langle\sigma\rangle_{\Phi}=(9.28 \pm 1.50) \times 10^{-39} \mathrm{~cm}^{2} / \mathrm{CH}_{2}$. Bottom: The relative cross-section error. The total systematic normalization error is $16.1 \%$. 

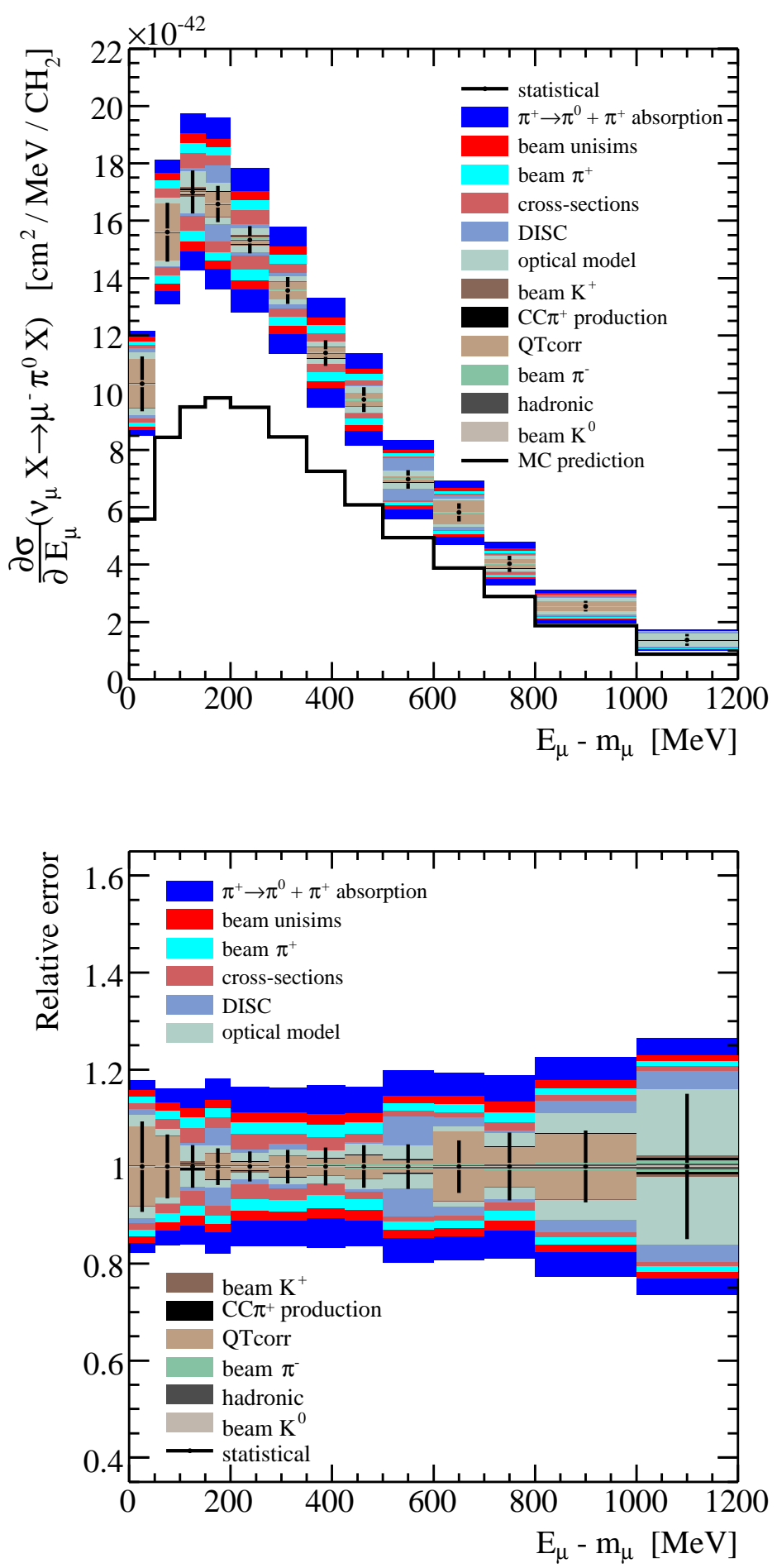

Figure 5.18: Top: The flux-averaged observable $\mathrm{CC} \pi^{0}$ cross-section differential in $E_{\mu}$ over the flux range $E_{\nu} \in(500-2000 \mathrm{MeV})$. The total flux-averaged cross-section calculated by integrating over $E_{\mu}$ is $\langle\sigma\rangle_{\Phi}=(9.20 \pm 1.47) \times 10^{-39} \mathrm{~cm}^{2} / \mathrm{CH}_{2}$. Bottom: The relative cross-section error. The total systematic normalization error is $15.8 \%$. 

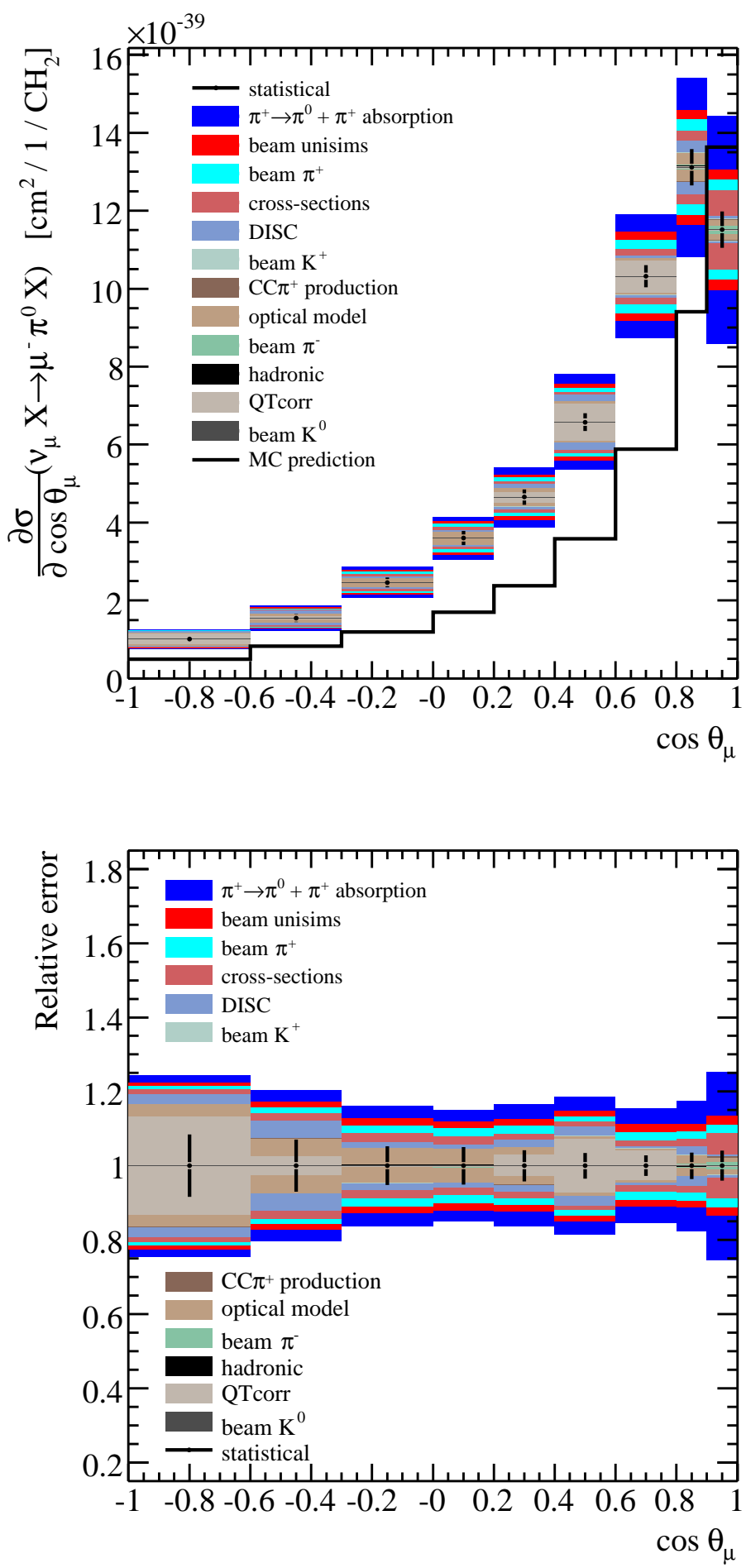

Figure 5.19: Top: The flux-averaged observable $\mathrm{CC} \pi^{0}$ cross-section differential in $\cos \theta_{\mu}$ over the flux range $E_{\nu} \in(500-2000 \mathrm{MeV})$. The total flux-averaged cross-section calculated by integrating over $\cos \theta_{\mu}$ is $\langle\sigma\rangle_{\Phi}=(9.10 \pm 1.50) \times 10^{-39} \mathrm{~cm}^{2} / \mathrm{CH}_{2}$. Bottom: The relative cross-section error. The total systematic normalization error is $17.4 \%$. 

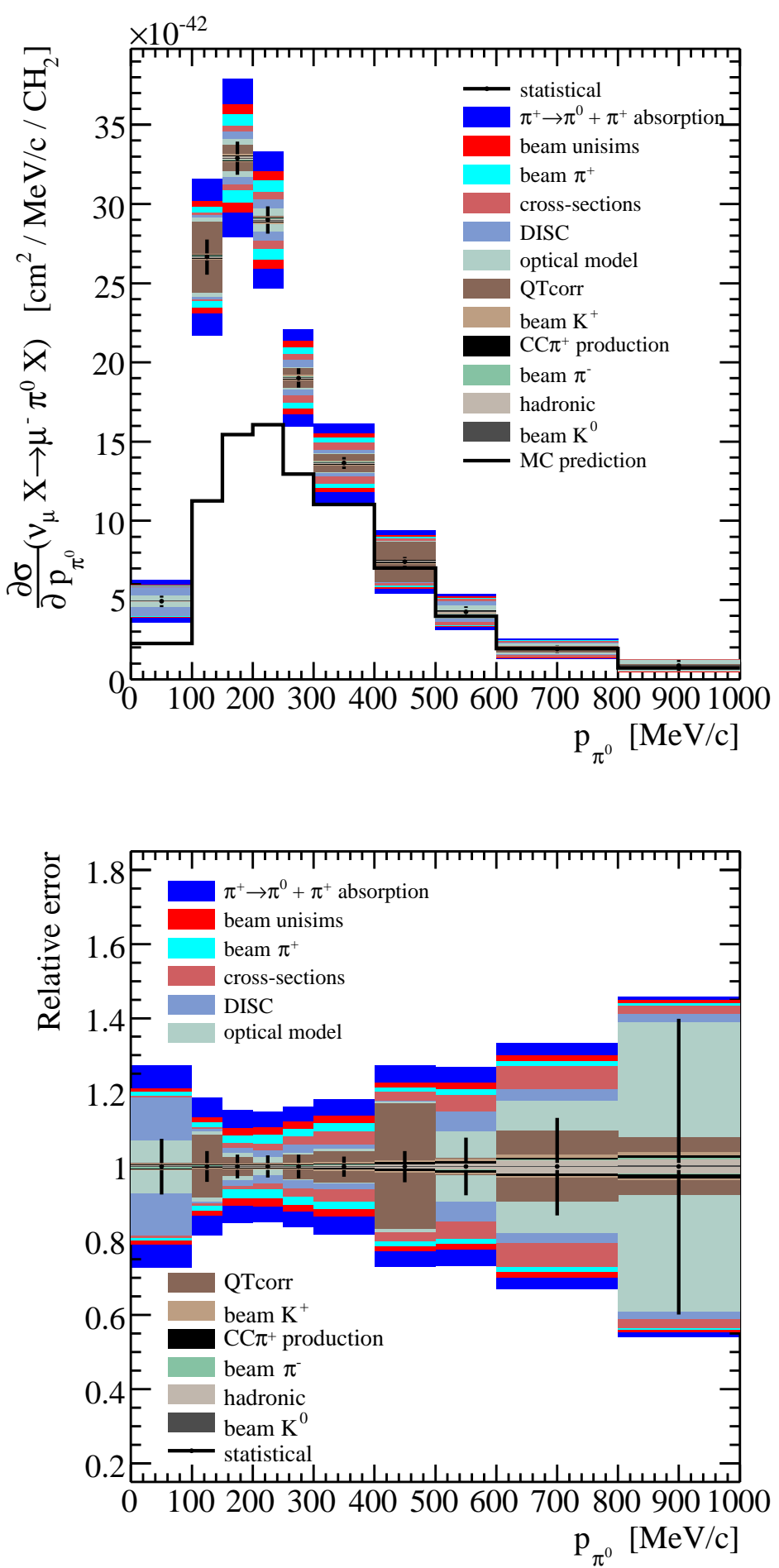

Figure 5.20: Top: The flux-averaged observable CC $\pi^{0}$ cross-section differential in $\left|\mathbf{p}_{\pi^{0}}\right|$ over the flux range $E_{\nu} \in(500-2000 \mathrm{MeV})$. The total flux-averaged cross-section calculated by integrating over $\left|\mathbf{p}_{\pi^{0}}\right|$ is $\langle\sigma\rangle_{\Phi}=(9.03 \pm 1.54) \times 10^{-39} \mathrm{~cm}^{2} / \mathrm{CH}_{2}$. Bottom: The relative cross-section error. The total systematic normalization error is $15.9 \%$. 

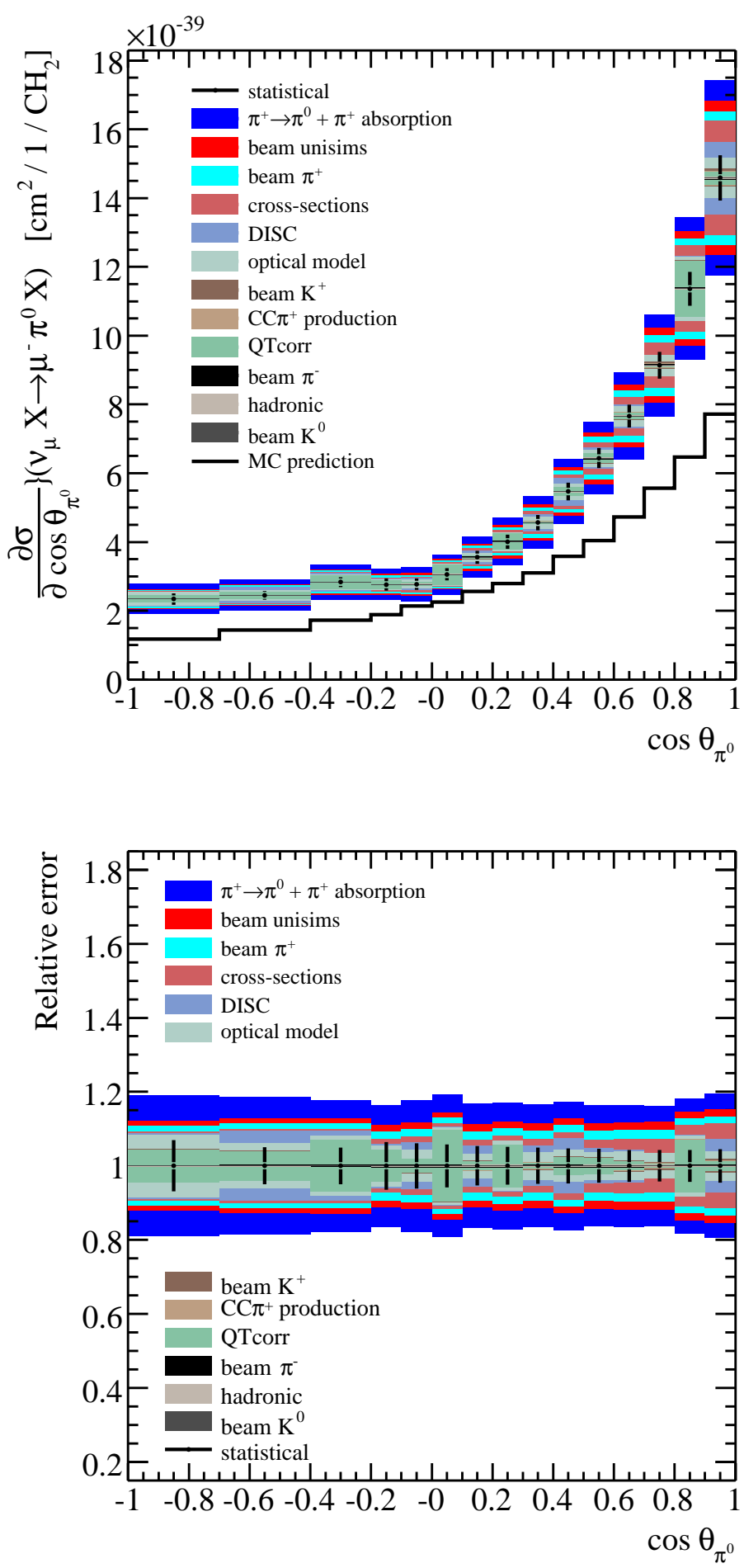

Figure 5.21: Top: The flux-averaged observable $\mathrm{CC} \pi^{0}$ cross-section differential in $\cos \theta_{\pi^{0}}$ over the flux range $E_{\nu} \in(500-2000 \mathrm{MeV})$. The total flux-averaged crosssection calculated by integrating over $\cos \theta_{\pi^{0}}$ is $\langle\sigma\rangle_{\Phi}=(9.54 \pm 1.55) \times 10^{-39} \mathrm{~cm}^{2} / \mathrm{CH}_{2}$. Bottom: The relative cross-section error. The total systematic normalization error is $16.3 \%$. 


\section{Chapter 6}

\section{Conclusions}

The study of neutrino interactions is an exciting field because of the myriad ways in which neutrinos can participate in physics beyond the Standard Model. There are right-handed neutrinos. Neutrinos have mass. Neutrinos oscillate; a quantum mechanical phenomenon where the flavor states are superpositions of the mass states. Neutrinos may also be their own anti-particles. All of these phenomena are active areas of research. Understanding these phenomena is helped by having a more complete understanding of neutrino interactions with nuclear matter.

The MiniBooNE experiment addressed a controversial neutrino oscillation signature while subsequently recording a large number of neutrino interactions over an energy range, $0.5-2.0 \mathrm{GeV}$. These interactions have allowed for a more complete understanding of neutrino interactions on a nuclear target. Previous experiments at these energies, were performed using hydrogen or deuterium targets, had much lower statistics, and did not report measurements for every interesting quantity.

To aid in the understanding of the intrinsic $\nu_{e}$ flux, an experiment was performed to understand the normalization of kaons produced during the proton-beryllium interactions from the target. While this experiment had backgrounds that were difficult to estimate, the upper limit set for the kaon normalization was consistent with fits to external kaon production data and of comparable precision.

This dissertation has presented the most comprehensive measurements of $\mathrm{CC} \pi^{0}$ 
interactions at neutrino energies $0.5-2.0 \mathrm{GeV}$. Additionally, they are also the first measurements on a nuclear target at these energies. The addition of this measurement to the measurements of $\mathrm{CCQE}, \mathrm{CC} \pi^{+}, \mathrm{NCEL}$, and $\mathrm{NC} \pi^{0}$, from the same experiment, will aid in the understanding of neutrino scattering off nuclear media.

This work presented 6 measurements characterizing $\mathrm{CC} \pi^{0}$ interactions: $\sigma\left(E_{\nu}\right)$, $\partial \sigma / \partial Q^{2}, \partial \sigma / \partial E_{\mu}, \partial \sigma / \partial \cos \theta_{\mu}, \partial \sigma / \partial\left|\mathbf{p}_{\pi^{0}}\right|$, and $\partial \sigma / \partial \cos \theta_{\pi^{0}}$. Each measurement was restricted to the flux range $E_{\nu} \in(0.5-2.0 \mathrm{GeV})$ enabling a combined measurement of the flux-averaged total cross-section $\langle\sigma\rangle_{\Phi}=\left(9.2 \pm 0.3_{\text {stat. }} \pm 1.5_{\text {sys. }}\right) \times 10^{-39} \mathrm{~cm}^{2} / \mathrm{CH}_{2}$ at average energy of $965 \mathrm{MeV}$.

These measurements show some qualitative disagreements with the Rein-Sehgal model. Disagreements are also observed in the other single pion modes. The obvious place for improvement would be that of the FSI model. The single largest source of uncertainty on the measurement, pion charge exchange and absorption in the mineral oil, came as no surprise. In fact, charge exchange and absorption in the target nucleus is a large part of the FSI uncertainty in the Rein-Seghal model. A more complete understanding of pion absorption and charge exchange would benefit the interpretation of these results.

It is hoped that these cross-sections will provide the motivation for better theoretical understanding of low energy neutrino interactions with nuclei. On their own, however, these measurements greatly improve the knowledge of $\mathrm{CC} \pi^{0}$ interactions. 


\section{Bibliography}

[1] W. Pauli, 1931.

[2] E. Fermi. An attempt of a theory of beta radiation. Z. Phys., 88:161, 1934.

[3] Frederick Reines and Clyde L. Cowan. Free Antineutrino Absorption Cross Section. I. Measurement of the Free Antineutrino Absorption Cross Section by Protons. Phys. Rev., 113(1):273-279, 1959.

[4] Bruce T. Cleveland et al. Measurement of the Solar Electron Neutrino Flux with the Homestake Chlorine Detector. Astrophysical Journal, 496(1):496, 1998.

[5] J. N. Abdurashitov et al. Measurement of the solar neutrino capture rate by the Russian-American gallium solar neutrino experiment during one half of the 22-year cycle of solar activity. J. Exp. Theor. Phys., 95:181-193, 2002.

[6] W. Hampel et al. GALLEX solar neutrino observations: Results for GALLEX IV. Phys. Lett., B447:127-133, 1999.

[7] Sheldon L. Glashow. Partial-symmetries of weak interactions. Nucl. Phys., 22(4):579 - 588, 1961.

[8] Steven Weinberg. A model of leptons. Phys. Rev. Lett., 19(21):1264-1266, Nov 1967.

[9] Abdus Salam. Weak and Electromagnetic Interactions. Originally printed in *Svartholm: Elementary Particle Theory, Proceedings Of The Nobel Symposium Held 1968 At Lerum, Sweden*, Stockholm 1968, 367-377.

[10] T.-P. Cheng and L.-F. Li. Gauge theory of elementary particle physics. Oxford Science Publications, 1984.

[11] F. Halzen and A. D. Martin. Quarks and Leptons: An Introductory Course in Modern Particle Physics. John Wiley \& Sons, Inc., 1984.

[12] G. P. Zeller. Private Communication.

[13] Y. Fukuda et al. Measurement of the Solar Neutrino Energy Spectrum Using Neutrino-Electron Scattering. Phys. Rev. Lett., 82(12):2430-2434, 1999. 
[14] B. Aharmim et al. Electron energy spectra, fluxes, and day-night asymmetries of $8 \mathrm{~B}$ solar neutrinos from measurements with $\mathrm{NaCl}$ dissolved in the heavy-water detector at the Sudbury Neutrino Observatory. Phys. Rev. C, 72(5):055502, Nov 2005 .

[15] Precision electroweak measurements on the Z resonance. Phys. Rep., 427(5-6):257 $-454,2006$.

[16] B. Pontecorvo. Mesonium and antimesonium. Sov. Phys. JETP, 6:429, 1957.

[17] Ziro Maki, Masami Nakagawa, and Shoichi Sakata. Remarks on the unified model of elementary particles. Prog. Theor. Phys., 28:870-880, 1962.

[18] Boris Kayser. On the Quantum Mechanics of Neutrino Oscillation. Phys. Rev., D24:110, 1981.

[19] Antonio Riotto. Theories of Baryogenesis. [hep-ph/9807454], 1998. A review.

[20] C. Amsler et al. Phys. Lett. B., 667:1, 2008.

[21] M. Apollonio et al. Search for neutrino oscillations on a long base-line at the CHOOZ nuclear power station. Eur. Phys. J., C27:331-374, 2003.

[22] E. Church et al. A proposal for an experiment to measure $\nu_{\mu} \rightarrow \nu_{e}$ oscillations and $\nu_{\mu}$ disappearance at the Fermilab Booster: BooNE. http://wwwboone.fnal.gov/publications/, 1997.

[23] C. Athanassopoulos et al. Candidate Events in a Search for $\bar{\nu}_{\mu} \rightarrow \bar{\nu}_{e}$ Oscillations. Phys. Rev. Lett., 75(14):2650-2653, Oct 1995.

[24] C. Athanassopoulos et al. Evidence for $\bar{\nu}_{\mu} \rightarrow \bar{\nu}_{e}$ Oscillations from the LSND Experiment at the Los Alamos Meson Physics Facility. Phys. Rev. Lett., 77(15):30823085, Oct 1996.

[25] C. Athanassopoulos et al. Results on $\nu_{\mu} \rightarrow \nu_{e}$ Neutrino Oscillations from the LSND Experiment. Phys. Rev. Lett., 81(9):1774-1777, Aug 1998.

[26] A. A. Aguilar-Arevalo et al. Evidence for neutrino oscillations from the observation of $\bar{\nu}_{e}$ appearance in a $\bar{\nu}_{\mu}$ beam. Phys. Rev., D64:112007, 2001.

[27] A. A. Aguilar-Arevalo et al. A Search for electron neutrino appearance at the $\Delta m^{2} \sim 1 \mathrm{eV}^{2}$ scale. Phys. Rev. Lett., 98:231801, 2007.

[28] A. A. Aguilar-Arevalo et al. A Search for Electron Antineutrino Appearance at the $\Delta m^{2} \sim 1 \mathrm{eV}^{2}$ Scale. Phys. Rev. Lett., 103:111801, 2009.

[29] A A. Aguilar-Arevalo et al. A search for muon neutrino and antineutrino disappearance in MiniBooNE. Phys. Rev. Lett., 103:061802, 2009.

[30] Fermilab Technical Memo TM-405, 1973.

[31] I. Stancu et al. Technical Design Report for the 8 GeV Beam. MiniBooNE-TDR: http://www-boone.fnal.gov/publications/, 2001. 
[32] I. Stancu et al. Technical Design Report for the MiniBooNE Neutrino Beam. MiniBooNE-TDR: http://www-boone.fnal.gov/publications/, 2001.

[33] A. A. Aguilar-Arevalo et al. The Neutrino Flux Prediction at MiniBooNE. Phys. Rev. D., 79:211801, 2009.

[34] Bartoszek Engineering. http://www.bartoszekeng.com/mboone/mboone.htm.

[35] I. Stancu et al. The MiniBooNE Detector Technical Design Report. MiniBooNETDR: http://www-boone.fnal.gov/publications/, 2001.

[36] Hamamatsu Photonics K.K., Electron Tube Division, 314-5, Shimokanzo, Toyooka-village, Iwata-gun, Shizuoka-ken, 438-01 Japan; US office: Hamamatsu Corporation, 360 Foothill Road, P.O. Box 6910, Bridgewater, N. J. 08807-1910.

[37] S. J. Brice et al. Photomultiplier tubes in the miniboone experiment. Nucl. Inst. Meth., 562(1):97 - 109, 2006.

[38] Hans-Otto Meyer. Measurement of Refraction, Dispersion, Density, and Thermal Expansion of Mineral Oil. MiniBooNE Internal MEMO, 2003.

[39] Ludox is a registered trademark of W. R. Grace Davison.

[40] A. A. Aguilar-Arevalo et al. The MiniBooNE Detector. Nucl. Inst. Meth. A, 599:28, 2009.

[41] S. Agostinelli et al. GEANT 4-A Simulation Toolkit. Nucl. Instrum. Meth., A506:250, 2003.

[42] R. J. Glauber. Lectures in Theoretical Physics, Volume 1. 1959.

[43] N. V. Mokhov et al. MARS Code Developments. [nucl-th/9812038], 1998.

[44] J. R. Sanford and C. L. Wang. BNL Note 11299, 1967.

[45] M. G. Catanesi et al. Measurement of the production cross-section of positive pions in the collision of $8.9 \mathrm{GeV} / \mathrm{c}$ protons on beryllium. European Physical Journal C, 52:29, 2007.

[46] I. Chemakin et al. Pion Production by Protons on a Thin Beryllium Target at 6.4, 12.3, and 17.5 GeV/c Incident Proton Momenta. Phys. Rev. C, 77:015209, 2008 .

[47] F. Abe et al. PRODUCTION OF NEUTRAL STRANGE PARTICLES K0(S) AND LAMBDA0 BY 12-GEV PROTONS ON NUCLEAR TARGETS. Phys. Rev., D36:1302-1319, 1987.

[48] Yu. D. Aleshin, I. A. Drabkin, and V. V. Kolesnikov. Production of $K^{ \pm}$Mesons from Be Targets at 62-Mrad at 9.5-GeV/c Incident Proton Momenta. ITEP-801977, 1977.

[49] T. Abbott et al. Measurement of particle production in proton-induced reactions at 14.6 gev/c. Phys. Rev. D, 45(11):3906-3920, 1992. 
[50] J. V. Allaby et al. CERN Report No. CERN 70-12. (unpublished).

[51] D. Dekkers et al. Experimental study of particle production at small angles in nucleon-nucleon collisions at 19 and 23 gev/c. Phys. Rev., 137(4B):B962-B978, 1965 .

[52] T. Eichten et al. Particle production in proton interactions in nuclei at $24 \mathrm{GeV} / \mathrm{c}$. Nuclear Physics B, 44(2):333 - 343, 1972.

[53] R. A. Lundy et al. $\pi^{ \pm}$and $K^{ \pm}$Production Cross Sections for 12.5-BeV Protons on Be. Phys. Rev. Lett., 14(13):504-507, 1965.

[54] G. J. Marmer et al. Differential Production Cross Sections of Low-Momentum Particles from 12.3-BeV/c Protons on Beryllium and Copper. Phys. Rev., 179(5):1294-1300, 1969.

[55] I. A. Vorontsov et al. A-DEPENDENCE OF FRAGMENTATION OF 9.2-GEV PROTONS ON NUCLEI. (IN RUSSIAN). 1988.

[56] R. Brun et al. GEANT3. CERN-DD/EE/84-1, 1987.

[57] D. Casper. The nuance neutrino physics simulation, and the future. Nucl. Phys. Proc. Suppl., 112:161-170, 2002.

[58] C. H. Llewellyn Smith. Neutrino Reactions At Accelerator Energies. Phys. Rept., $3: 261,1972$.

[59] G. P. Zeller. Low Energy Neutrino Cross Sections: Comparison of Various Monte Carlo Predictions to Experimental Data. [hep-ex/0312061], 2003.

[60] V. Bernard et al. J. Phys. G, 28:R1, 2002.

[61] A. A. Aguilar-Arevalo et al. First Measurement of the Muon Neutrino Charged Current Quasielastic Double Differential Cross Section. Submitted to Phys. Rev. D: [hep-ex/1002.2680].

[62] D. Rein and L.M. Sehgal. Neutrino Excitation of Baryon Resonances and Single Pion Production. Annals of Physics, 133:79, 1981.

[63] Kislinger, M. and Feynman, R. P. and Ravndal, F. Current Matrix Elements from a Relativistic Quark Model. Phys. Rev. D, 3:2706, 1971.

[64] R. A. Smith and E. J. Moniz. NEUTRINO REACTIONS ON NUCLEAR TARGETS. Nucl. Phys., B43:605, 1972.

[65] Hans-Otto Meyer. Index of Refraction of Marcol 7. MiniBooNE-Technical-Note90, 2003.

[66] P. A. Cherenkov. Visible Emission of Clean Liquids by Action of Radiation. Doklady Akad. Nauk SSSR, 2:451, 1934.

[67] I. Tamm. Radiation emitted by uniformly moving electrons. J. Phys. U.S.S.R, 1:439, 1939. 
[68] Thomas Erber and Heimo G Latal. Unified radiation formulae for classical and quantum electrodynamics. European Journal of Physics, 24(1):67-79, 2003.

[69] A. A. Aguilar-Arevalo et al. Measurement of muon neutrino quasi-elastic scattering on carbon. Phys. Rev. Lett., 100:032301, 2008.

[70] T. Katori. A MEASUREMENT OF THE MUON NEUTRINO CHARGED CURRENT QUASIELASTIC INTERACTION AND A TEST OF LORENTZ VIOLATION WITH THE MINIBOONE EXPERIMENT. PhD thesis, Indiana University, 2008.

[71] M. J. Wilking. Measurement of Neutrino Induced, Charged Current, Charged Pion Production. PhD thesis, University of Colorado, Boulder, 2009.

[72] A. A. Aguilar-Arevalo et al. Measurement of Neutrino-Induced Charged-Current Charged Pion Production. In preparation.

[73] D. C. Cox. A Measurement of the Neutral Current Neutrino-Nucleon Elastic Cross Section at MiniBooNE. PhD thesis, Indiana University, 2008.

[74] D. Perevalov. Neutrino-Nucleus Neutral Current Elastic Interactions Measurement in MiniBooNE. PhD thesis, University of Alabama, 2009.

[75] A. A. Aguilar-Arevalo et al. Measurement of $\nu_{\mu}$ and $\bar{\nu}_{\mu}$ induced neutral current single $\pi^{0}$ production cross sections on mineral oil at $E_{\nu} \sim O(1 \mathrm{GeV})$. Phys. Rev. D., 81:013005, 2010 .

[76] E. D. Zimmerman. Granularity of the LMC Fiber Hodoscopes. MiniBooNETechnical-Note-72, 2002.

[77] R. B. Patterson et al. The extended-track reconstruction for MiniBooNE. Nucl. Inst. Meth. A, 608:206, 2009.

[78] R. B. Patterson. A Search for Muon Neutrino to Electron Neutrino Oscillations at $\Delta m^{2}>0.1 \mathrm{eV}^{2}$. PhD thesis, Princeton University, 2007.

[79] I. McLaren. MINUIT - Function Minimization and Error Analysis. CERN Program Library, D506, 1998.

[80] E. B. Saff and A. B. J. Kuijlaars. Distributing many points on a sphere. The Mathematical Intelligencer, 19(1):5-11, 1997.

[81] I. Stancu. Studies of the Charge Likelihood for a Perfect BooNE Detector. MiniBooNE-Technical-Note-5, 1998.

[82] I. Stancu. Maximum Likelihood Event Reconstruction for BooNE: Point-like Particles in a Perfect Detector. MiniBooNE-Technical-Note-6, 1998.

[83] I. Stancu. The Underlying Parameters of the Maximum Likelihood Event Reconstruction in MiniBooNE. MiniBooNE-Technical-Note-36, 2001.

[84] I. Stancu. An Introduction to the Maximum Likelihood Event Reconstruction in MiniBooNE. MiniBooNE-Technical-Note-50, 2002. 
[85] S. B. Barish et al. Study of neutrino interactions in hydrogen and deuterium. II. Inelastic charged-current reactions. Phys. Rev. D., 19(9):2521-2542, 1979.

[86] G. M. Radecky et al. Study of single-pion production by weak charged currents in low-energy $\nu d$ interactions. Phys. Rev. D., 25(5):1161-1173, 1982.

[87] T. Kitagaki et al. Charged-current exclusive pion production in neutrinodeuterium interactions. Phys. Rev. D., 34(9):2554-2565, 1986.

[88] H. J. Grabosch et al. Cross-section measurements of single pion production in charged current neutrino and antineutrino interactions. Zeit. Phys. C., 41(4):527$531,1989$.

[89] D. Allasia et al. Investigation of Exclusive Channels in $\nu / \bar{\nu}$-Deuteron Charged Current Interactions. Nucl. Phys. B., 343:285-309, 1990.

[90] H. Gallagher. The NEUGEN neutrino event generator. Nucl. Phys. B Proc. Supp., 112:188-194, 2002.

[91] L. M. Seghal. Proceedings of the European Physical Society International Conference on High Energy Physics, page 98, 1980.

[92] R. Tayloe. Private Communication.

[93] Glen Cowan. Statistical Data Analysis. Oxford Science Publications, 1998.

[94] G. D'Agostini. A multidimensional unfolding method based on Bayes' theorem. Nucl. Instrum. Meth., A362:487-498, 1995.

[95] R. B. Patterson. A Brief Description of the DISC and QTCORR unisims. MiniBooNE Internal MEMO, 2007.

[96] D. W. Schmitz. A measurement of hadron production cross-sections for the simulation of accelerator neutrino beams and a search for muon neutrino to electron neutrino oscillations in the $\Delta m^{2} \sim 1 \mathrm{eV}^{2}$ region. PhD thesis, Columbia University, 2008.

[97] C. Ashery et al. True Absorption and Scattering of Pions on Nuclei. Phys. C, 23:2173, 1981.

[98] G. Battistoni et al. The FLUKA code: Description and benchmarking. AIP Conf. Proc., 896:31, 2007.

[99] C. Zeitnitz and Gabriel T. A. The GEANT-CALOR Interface and Benchmark Calculations of ZEUS Test Calorimeters. Nucl. Instrum. Meth., A349:106, 1994.

[100] L. Lyons et al. How to Combine Correlated Estimates of a Single Physical Quantity. Nucl. Instr. Meth., A270:110-117, 1988.

[101] C. Anderson. Measurement of the neutral current $\pi^{0}$ production absolute cross section. MiniBooNE-Technical-Note-272, 2009. 


\section{Appendix A}

\section{Unfolding}

The purpose of unfolding is to take a distribution of a measured estimate for a quantity and form an estimator for that underlying quantity. Typically this is attempted through a MC simulation incorporating detector responses and physical processes that can smear a reconstruction.

A background subtracted measured observable, $\tilde{\mathrm{x}}$, is posited to come from an underlying true distribution, x. Since these measurements are performed during a finite interval of time, they are binned within some interval and are assumed to be averaged over each bin $^{1}$. Barring extremely low statistics, this is how we do business. Therefore, these quantities (true and measured) can be represented as vectors with each index representing a bin along that interval. Attempting to map $\tilde{\mathbf{x}} \mapsto \mathbf{x}$ requires knowledge of the processes that can migrate events from one bin into another. While there are many ways one could study this migration, typically a MC is produced that incorporates the smearing associated with the detection device, and physics processes that affect the outcome of the measurement. This mapping, $\mathbf{R}$, is referred to as the response matrix. For this discussion the convention will be that the first index corresponds to the true vector, and the second index corresponds to the reconstructed vector.

The migration of true bins into reconstructed bins matrix, $\mathbf{B}$, is formed by taking

\footnotetext{
${ }^{1}$ There is no a priori reason for the measured and true distributions to have the same binning.
} 
the transpose of the column-normalized response matrix. Explicitly:

$$
B_{i j}=\left(\frac{R_{i j}}{\sum_{k} R_{i k}}\right)^{T}=\frac{R_{j i}}{\sum_{k} R_{i k}} .
$$

The bin migration matrix gives the probability that a given bin of the true distribution gets mapped to the reconstructed distribution.

While there are probably an infinite number of ways to unfold a measured distribution, the goal of this memo is not to discuss the various ways to do so. The goal is to discuss the propagation of the statistical errors. For argument sake, assume that a method of unfolding is chosen. Then the inferred true distribution is found by:

$$
\hat{x}_{i}=\sum_{j} U_{i j} \tilde{x}_{j}
$$

where $\hat{\mathbf{x}}$ is an estimator for the true distribution $\mathbf{x}$.

\section{A.1 Bayesian Unfolding}

The chosen method of unfolding involves a method derived from Bayes' theorem [93, 94]. Bayes' theorem states:

$$
P(A \mid B) P(B)=P(B \mid A) P(A)
$$

relating the probability of a given series of causes, $A$, to events, $B$. The probability of a cause producing a given event is

$$
P\left(B_{j} \mid A_{i}\right)=\frac{P\left(A_{i} \mid B_{j}\right) P\left(B_{i}\right)}{\sum_{k} P\left(A_{i} \mid B_{k}\right) P\left(B_{k}\right)}
$$

which is recognized as the migration matrix, $\mathbf{B}$, in Eqn. A.1. Reversing the problem to infer the probability that a given measured event came from a cause is given by

$$
P\left(A_{i} \mid B_{j}\right)=\frac{P\left(B_{j} \mid A_{i}\right) P\left(A_{i}\right)}{\sum_{k} P\left(B_{j} \mid A_{k}\right) P\left(A_{k}\right)}
$$

and is defined to be the Bayesian unsmearing matrix. The major difference between these two probabilities are the priors necessary to calculate these matrices. The bin 
migration matrix is independent of the distribution of causes. Whereas, the Bayesian unsmearing matrix is biased toward the initial distribution of causes. While the migration matrix is the transpose of the column normalized response matrix, it can be seen that the Bayesian unsmearing matrix is the row normalized response matrix or:

$$
U_{i j}=\frac{R_{i j}}{\sum_{k} R_{k j}} \equiv \frac{R_{i j}}{N_{j}}
$$

\section{A.2 Statistical Error Propagation}

The propagation of statistical error from both the data and MC statistics is handled through a Jacobian transformation. Explicitly:

$$
\mathbf{V}(\hat{\mathbf{x}})=\left(\frac{\partial \hat{\mathbf{x}}}{\partial \tilde{\mathbf{x}}}\right) \mathbf{V}(\tilde{\mathbf{x}})\left(\frac{\partial \hat{\mathbf{x}}}{\partial \tilde{\mathbf{x}}}\right)^{T}+\left(\frac{\partial \hat{\mathbf{x}}}{\partial \mathbf{U}}\right)\left(\frac{\partial \mathbf{U}}{\partial \mathbf{R}}\right) \mathbf{V}(\mathbf{R})\left(\frac{\partial \mathbf{U}}{\partial \mathbf{R}}\right)^{T}\left(\frac{\partial \hat{\mathbf{x}}}{\partial \mathbf{U}}\right)^{T}
$$

where $\mathbf{V}(\tilde{\mathbf{x}})$ and $\mathbf{V}(\mathbf{R})$ are the statistical covariance matrices of $\tilde{\mathbf{x}}$ and $\mathbf{R}$ respectively. The first term of Eqn. A.7 handles the data statistics, and the second handles the effect of MC statistics. This is completely general for any chosen unfolding method assuming that the relationship between the unfolding and response matrices are calculable. This equation can be expressed per matrix element as

$$
V_{i j}(\hat{\mathbf{x}})=\sum_{k, l}\left(\frac{\partial \hat{x}_{i}}{\partial \tilde{x}_{k}}\right) V_{k l}(\tilde{\mathbf{x}})\left(\frac{\partial \hat{x}_{j}}{\partial \tilde{x}_{l}}\right)+\sum_{\ldots}\left(\frac{\partial \hat{x}_{i}}{\partial U_{k l}}\right)\left(\frac{\partial U_{k l}}{\partial R_{m n}}\right) V_{m n p r}(\mathbf{R})\left(\frac{\partial U_{s t}}{\partial R_{p r}}\right)\left(\frac{\partial \hat{x}_{j}}{\partial U_{s t}}\right)
$$

where the second summation is over all the repeated indices. From this point onward this Eqn. A.8 will be applied to the Bayesian unfolding method. The complete expression for all pieces of Eqn. A.8 are calculated as:

$$
\begin{aligned}
\frac{\partial \hat{x}_{i}}{\partial \tilde{x}_{k}} & =U_{i k} \\
\frac{\partial \hat{x}_{i}}{\partial U_{k l}} & =\delta_{i k} \tilde{x}_{l} \\
\frac{\partial U_{k l}}{\partial R_{m n}} & =\frac{\delta_{l n}}{N_{l}}\left(\delta_{k m}-U_{k l}\right) \\
V_{k l}(\tilde{\mathbf{x}}) & =\sigma_{k}^{2}(\tilde{\mathbf{x}}) \delta_{k l} \\
V_{m n p r}(\mathbf{R}) & =R_{m n} \delta_{m p} \delta_{n r}
\end{aligned}
$$


where $\delta_{a b}$ are Kronecker delta functions, $\sigma_{k}^{2}(\tilde{\mathbf{x}})$ is left explicit ${ }^{2}$, and the statistical error of $\mathbf{R}$ is assumed to be Poisson. The statistical errors on the MC and folded distribution are diagonal which is expressed by the Kronecker deltas. Plugging all the terms into Eqn. A.8, the statistical covariance matrix for Bayesian unfolding is

$$
V_{i j}(\hat{\mathbf{x}})=\sum_{k}^{b i n s}\left\{U_{i k} \sigma_{k}^{2}(\tilde{\mathbf{x}}) U_{j k}+\frac{\tilde{x}_{k}^{2} U_{i k}^{2}}{R_{i k}}\left(\delta_{i j}-U_{j k}\right)\right\} .
$$

While it may not immediately look it, $V_{i j}(\hat{\mathbf{x}})$ is symmetric with an interchange of $i \leftrightarrow j$ as was required by construction.

\section{A.3 Unfolding Closure Tests}

To verify that the code is working the unfolding procedure is subjected to two tautology tests. The first test simply unfolds the same reconstructed distribution that the unfolding was trained on. This should return a distribution that is identical to the true distribution that was used in the unfolding. Fig. A.1 shows the bin migration, unfolding, and response matrices along with the true, reconstructed, and unfolded distributions in neutrino energy as an example. The unfolding has satisfied this tautology test.

The second test comes from the realization that the true distribution should be an eigen-distribution of unfolding matrix times the bin migration matrix. Or that,

$$
\mathbf{x}=(\mathbf{U} \cdot \mathbf{B}) \cdot \mathbf{x}
$$

where $\mathbf{x}$ is the true distribution, $\mathbf{U}$ is the Bayesian unfolding matrix, and $\mathbf{B}$ is the bin migration matrix. Fig. A.2 shows the product of the two matrices, the true distribution, and the product of the matrices times the true distribution. Indeed, the true distribution is an eigen-distribution of the matrix. These tests proved to be excellent ways to find bugs coded into the methods.

\footnotetext{
2 The reason being is that one tends to use distributions that have some sort of normalization applied and the scaling has to be taken into account. If not then this is just the Poisson errors, $\sigma_{k}^{2}(\tilde{\mathbf{x}})=\tilde{x}_{k}$.
} 

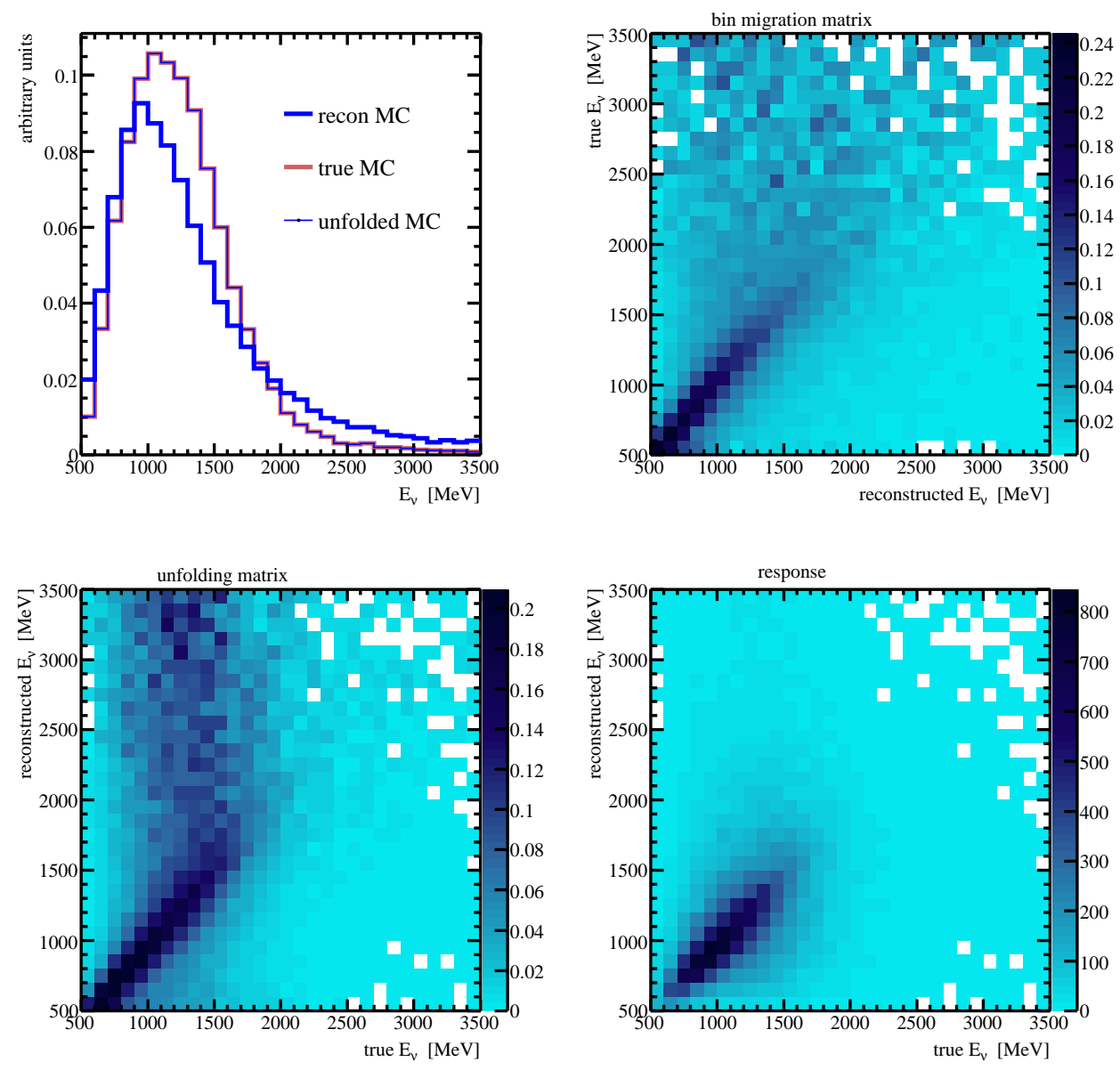

Figure A.1: Top left: The true, reconstructed, and unfolded $E_{\nu}$ distributions. The unfolded distribution is identical to the true distribution as expected. Top Right: The bin migration matrix formed by the transpose of the column normalized response matrix. Bottom Left: The Bayesian unfolding matrix defined as the row normalized response matrix. Bottom Right: The response matrix.

\section{A.4 Multi-dimensional Unfolding}

The technique of unfolding may also be applied to multi-dimensional distributions. The procedure is straight forward, but can be rather tricky in its implementation. An unfolding for an arbitrary number of dimensions is an extension of Eqn. A.2 given by,

$$
\hat{x}_{i_{1} \ldots i_{N}}=\sum_{j_{1} \ldots j_{N}} U_{i_{1} \ldots i_{N}, j_{1} \ldots j_{N}} \tilde{x}_{j_{1} \ldots j_{N}}
$$



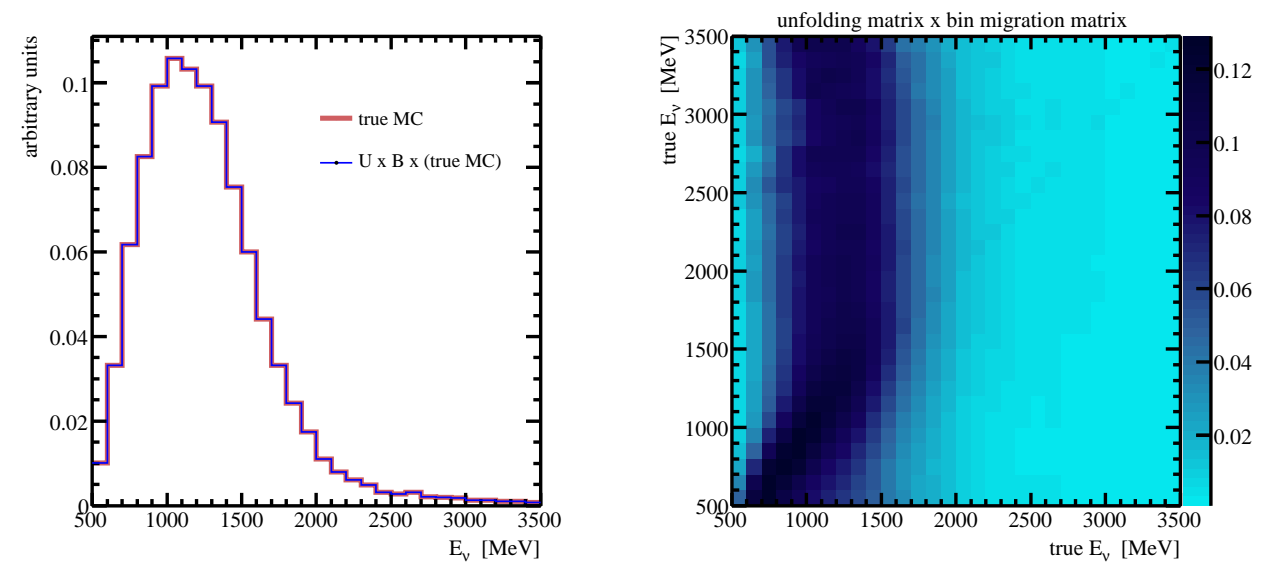

Figure A.2: Right: The product of the unfolding times the bin migration matrix. Left: The true distribution and the unfolding times bin migration times the true distribution. The true distribution is an eigen-distribution of this matrix.

where $\hat{x}$ and $\tilde{x}$ each have dimensionality $N, i_{1} \ldots i_{N}$ represents the indices over the number of dimensions for the true variables, $j_{1} \ldots j_{N}$ represents the indices over the reconstructed variables, and $U$ has dimensionality $2 N$. As these matrices can get computationally cumbersome, this equation can be expressed exactly as Eqn. A.2 by using a simple trick used in the computer sciences. All multi-dimensional arrays on a computer are stored as one-dimensional arrays. The additional indices are just a notational convenience for the user. This means that a mapping of $\left\{i_{1} \ldots i_{N}\right\} \mapsto I$ can be constructed where $I$ is a unique global index representing all bins of every dimension ${ }^{3}$. The mapping is given in general by,

$$
I=\left(i_{1}+n_{1}\left(i_{2}+n_{2}\left(i_{3}+\ldots\left(i_{N-1}+n_{N-1} i_{N}\right) \ldots\right)\right.\right.
$$

where $n_{i}$ is the number of bins in that dimension. It should be noted that the integral bins at the edges of each dimension must be included, sometimes referred to as the over(under)flows. With this transformation Eqn. A.16 becomes,

$$
\hat{x}_{I}=\sum_{J} U_{I J} \tilde{x}_{J}
$$

\footnotetext{
${ }^{3}$ This trick works because each dimension has a finite number of bins.
} 
which is recognized as Eqn. A.2 with a substitution of indices. All of the methods derived for the one-dimensional case in this appendix are completely valid for an arbitrary number of dimensions when expressed in this form. After the unfolding procedure, the mapping can be inverted to give the unfolded distribution.

\section{A.5 Remarks}

This calculation came about from the realization that the propagation of statistical errors in an unfolding method would lead to a covariance matrix with off diagonal terms. Therefore, the proper handling of the total error of a distribution involves adding this full matrix to the measured systematic error matrix. Also, even in cases of large MC statistics, the relative error on portions of the unfolding matrix can still be large. Additionally, the effect of unsmearing leads to an apparent reduction of statistical errors on the diagonal elements. This is because smearing forces correlation between the unfolded bins. Finally, a more general discussion for other unfolding methods are discussed in Ref. [101]. That discussion was extended to arrive at a closed form solution for Bayesian unfolding. 


\section{Appendix B}

\section{Cross-section Tabulations}

The cross-sections presented in Chapter 5 are tabulated in this appendix. Measurements that were presented in units proportional to $\mathrm{MeV}$ are reported proportional to $\mathrm{GeV}$ in the tables. The cross-sections presented are:

- The total observable $\mathrm{CC} \pi^{0}$ cross-section as a function of neutrino energy (Fig. 5.16 and Table B.1).

- The flux-averaged observable $\mathrm{CC} \pi^{0}$ cross-section differential in $Q^{2}$ over the flux range $E_{\nu} \in(500-2000 \mathrm{MeV})$ (Fig. 5.17 and Table B.2).

- The flux-averaged observable $\mathrm{CC} \pi^{0}$ cross-section differential in $E_{\mu}$ over the flux range $E_{\nu} \in(500-2000 \mathrm{MeV})$ (Fig. 5.18 and Table B.3).

- The flux-averaged observable $\mathrm{CC} \pi^{0}$ cross-section differential in $\cos \theta_{\mu}$ over the flux range $E_{\nu} \in(500-2000 \mathrm{MeV})$ (Fig. 5.19 and Table B.4).

- The flux-averaged observable $\mathrm{CC} \pi^{0}$ cross-section differential in $\left|\mathbf{p}_{\pi^{0}}\right|$ over the flux range $E_{\nu} \in(500-2000 \mathrm{MeV})$ (Fig. 5.20 and Table B.5).

- The flux-averaged observable $\mathrm{CC} \pi^{0}$ cross-section differential in $\cos \theta_{\pi^{0}}$ over the flux range $E_{\nu} \in(500-2000 \mathrm{MeV})$ (Fig. 5.21 and Table B.6). 


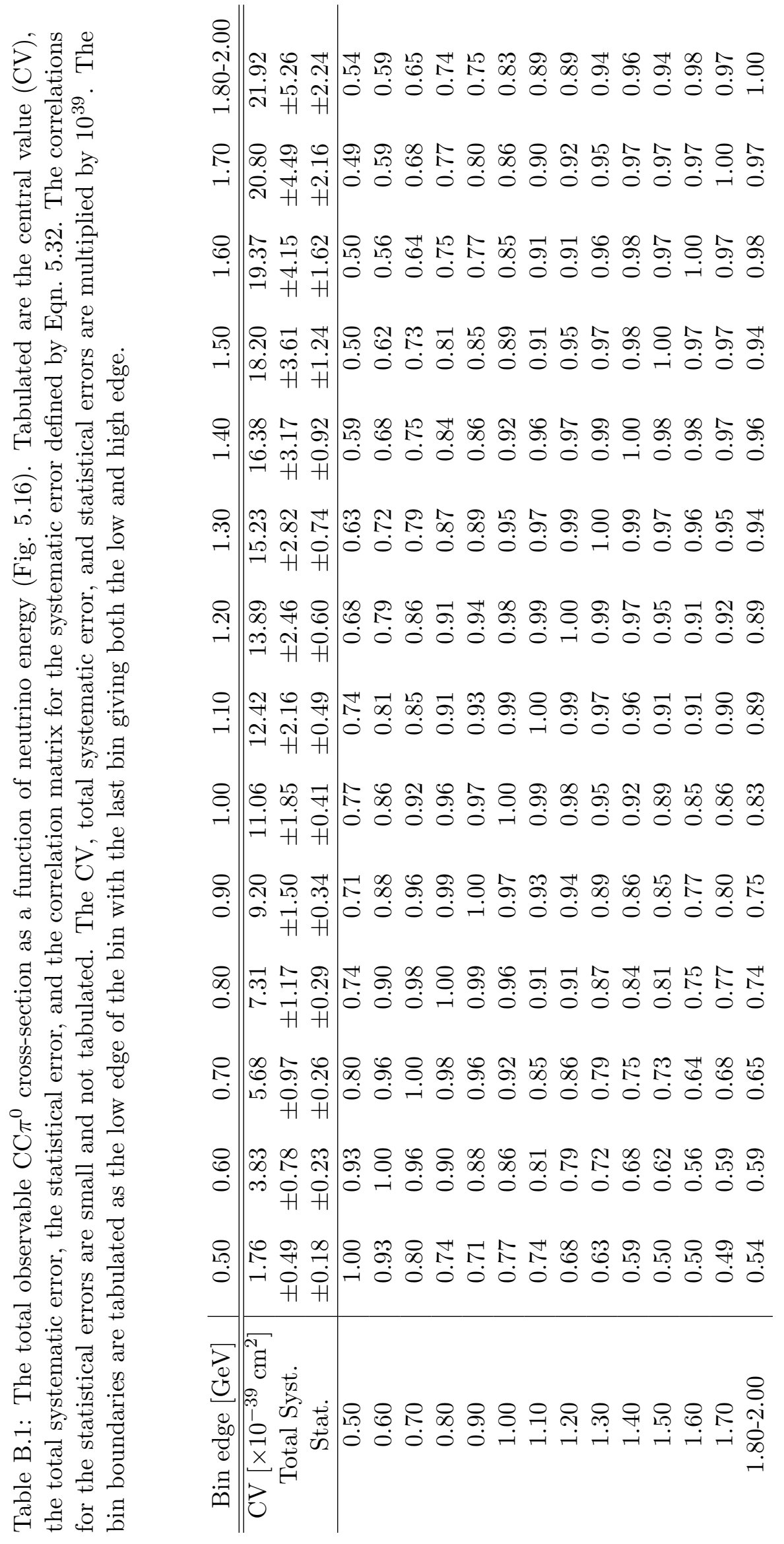


芥句

10. 0 .

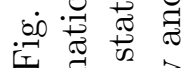

近 릉

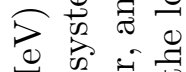

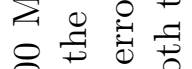

$8+00$

N

।

8 贾

$\psi$ च त $\overrightarrow{0}$

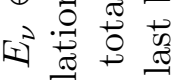

80

त्र 0 \&

岁焉

Ð

䨔

के

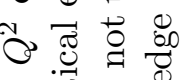

$\exists$.

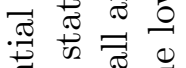

过可

का क

苛完

รี

을

政

点 $\begin{gathered}0 \\ 0\end{gathered}$

0 完

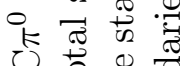

ర己

웡

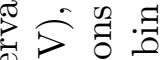

它要

○ $\frac{\pi}{0}$

D.

정

氙要

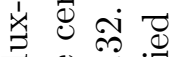

E

焉家

สี II

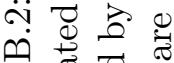

中心 芯

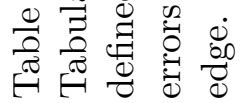

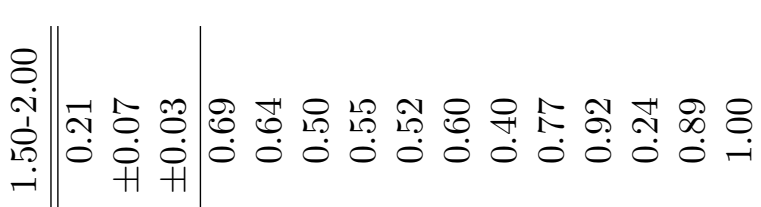

กิ

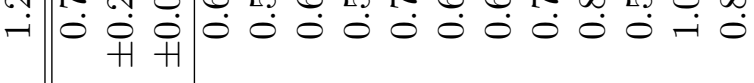

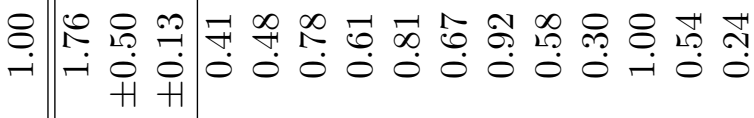

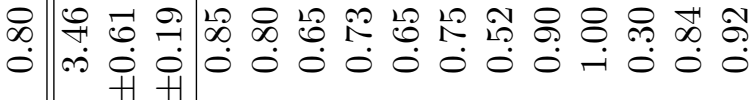

R

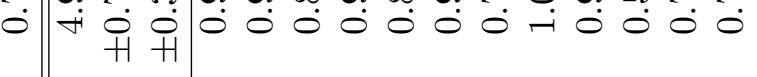

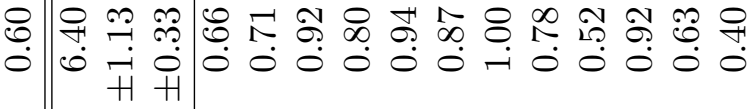

ำ 유 ำ

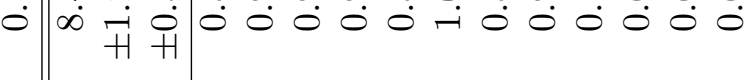

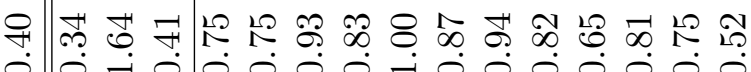

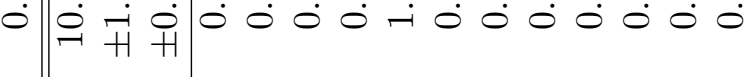

๑

$\dot{0}$ 需

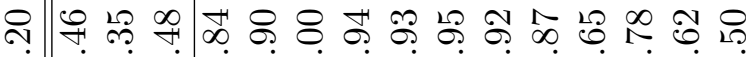

$\because \mid \forall$ i

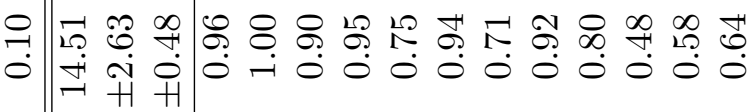

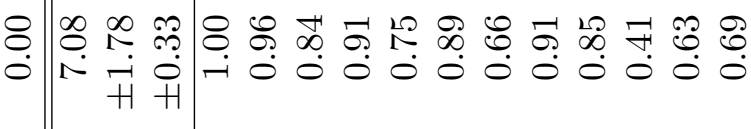




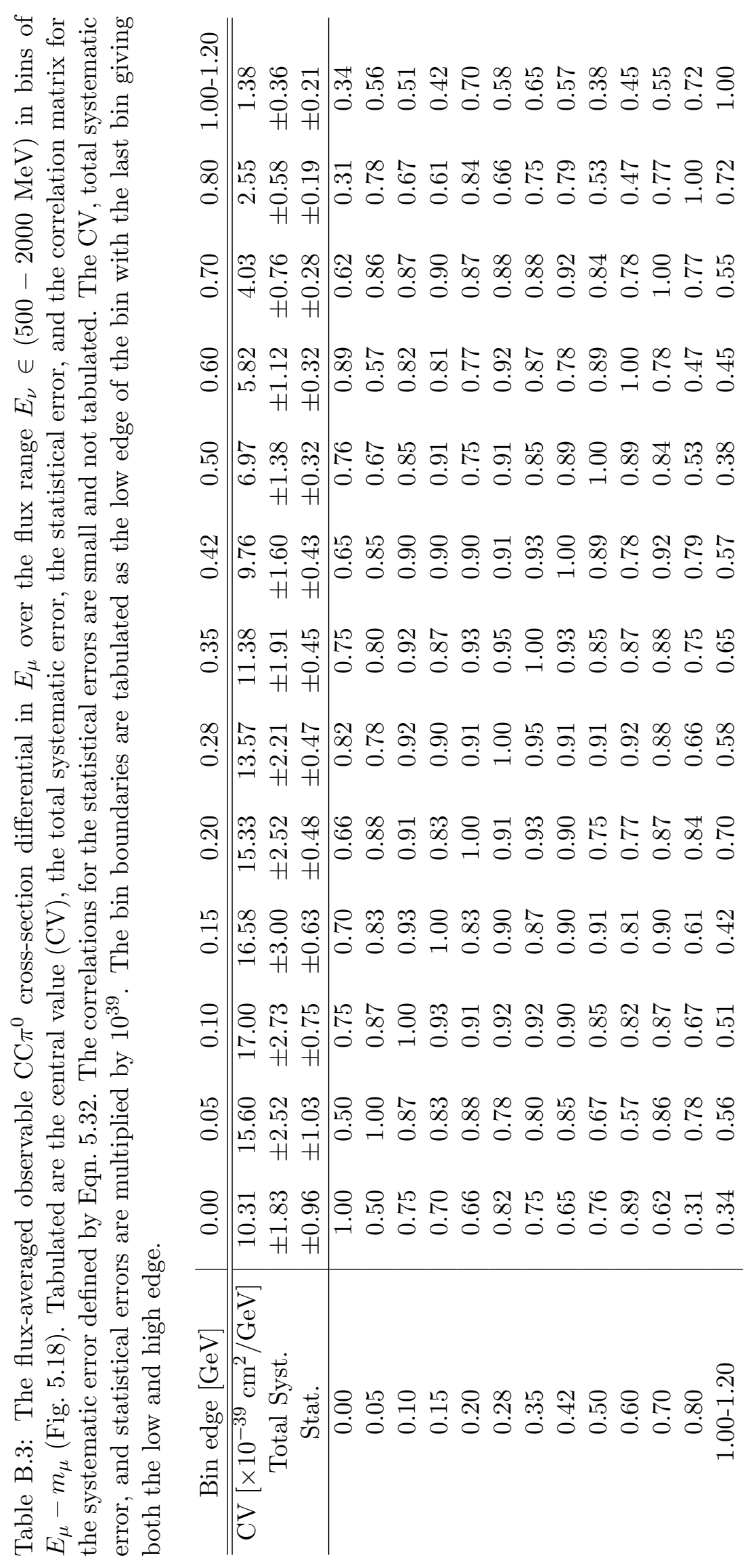




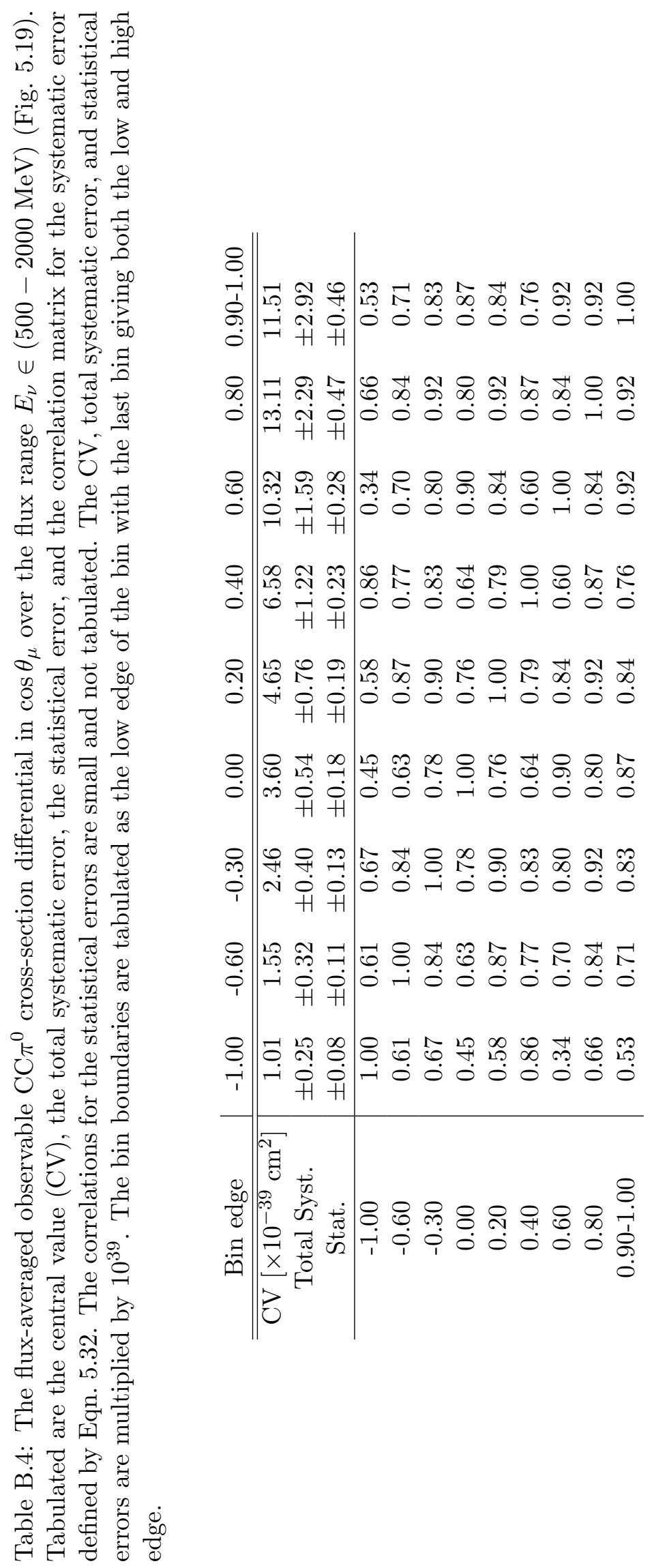




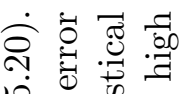

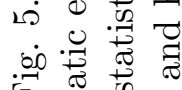

I

$\approx$ 贾

की

ᄋ해

ণิ

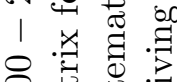

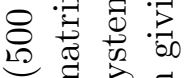

य है

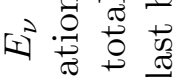

$\infty_{0}>0$

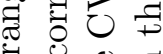

兽焉青

ङ च्च

む)

ठ

알.

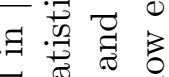

范

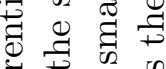

Ð艹

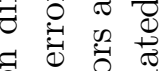

일

范

运.

完

战范

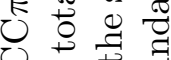

웜

동

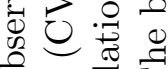

$6 \frac{\pi}{0}$

จ.

저

焉要

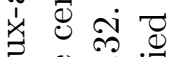

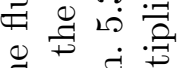

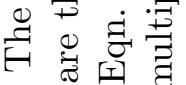

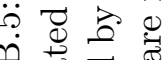

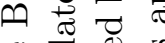

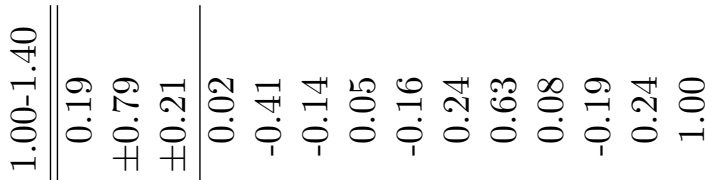

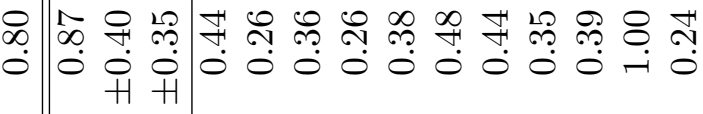

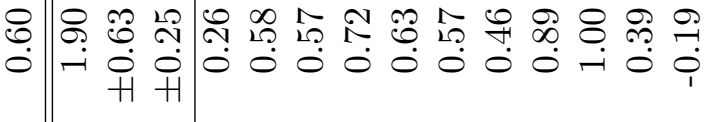

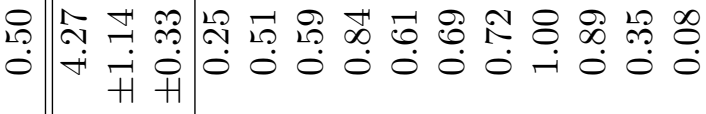

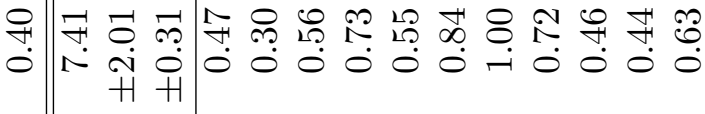

•

0 m

คุ

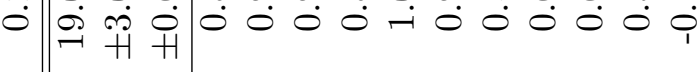

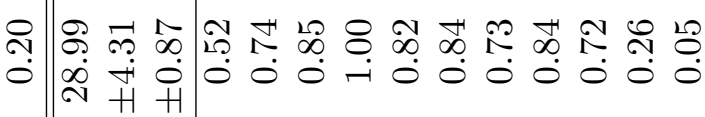

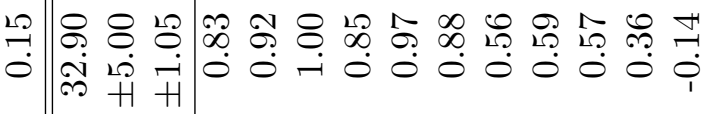

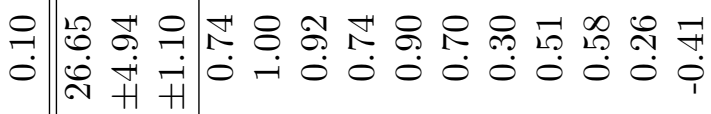

\&

䨔 


\begin{tabular}{|c|c|c|c|}
\hline 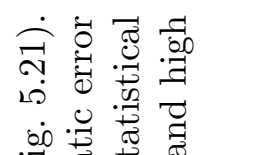 & $\begin{array}{l}\overrightarrow{1} \\
\dot{1} \\
\stackrel{0}{0}\end{array}$ & 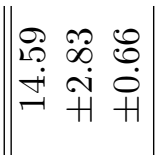 & 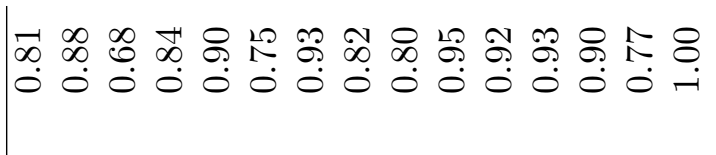 \\
\hline 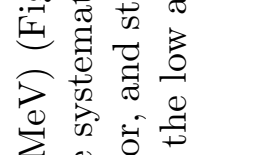 & $\stackrel{\odot}{\infty}$ & 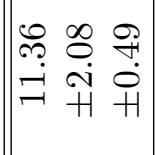 & 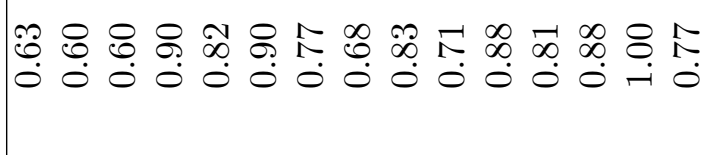 \\
\hline 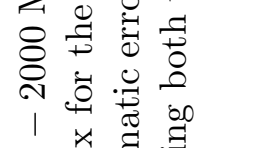 & 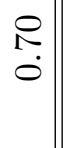 & 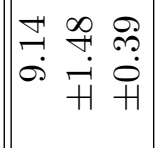 & 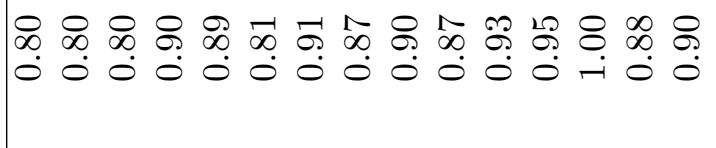 \\
\hline 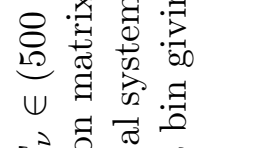 & 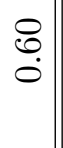 & 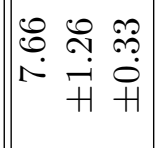 & 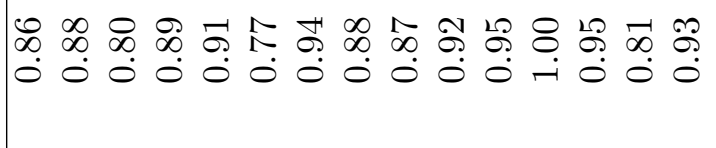 \\
\hline : & 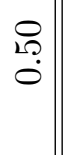 & 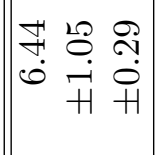 & 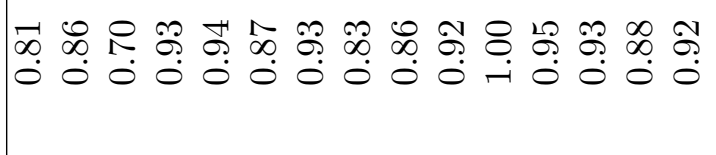 \\
\hline 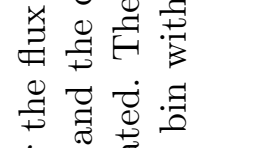 & $\stackrel{\circ}{\stackrel{+}{\circ}}$ & 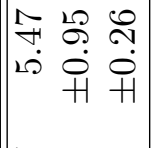 & 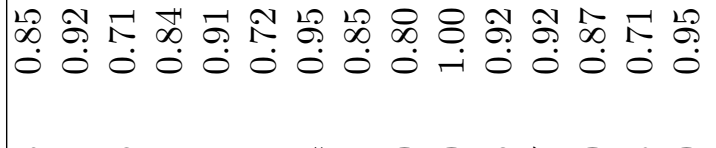 \\
\hline 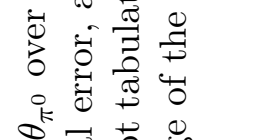 & 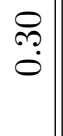 & 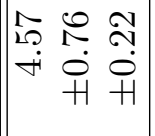 & 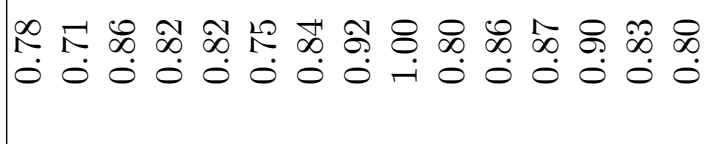 \\
\hline 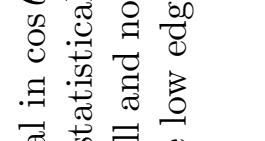 & 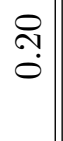 & 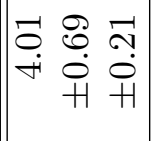 & 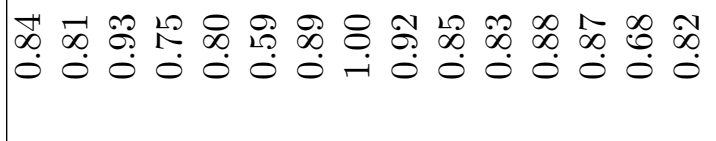 \\
\hline 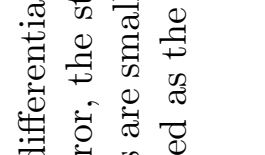 & $\stackrel{\circ}{?} \stackrel{1}{\circ}$ & 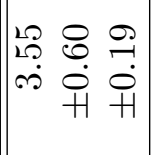 & 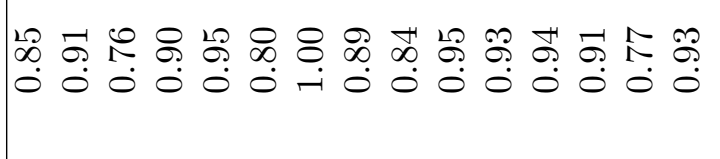 \\
\hline $\begin{array}{l}0 \\
0 \\
0\end{array}$ & $\stackrel{8}{\odot}$ & $\left|\begin{array}{lll}0 & 0 & \infty \\
0 & 0 & \cdots \\
\dot{0} & 0 & 0 \\
& + & +1 \\
& +1 & +1\end{array}\right|$ & 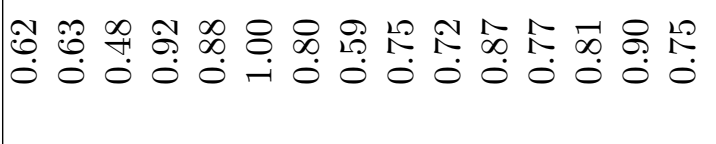 \\
\hline 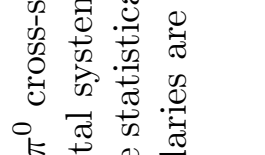 & \begin{tabular}{l||}
0 \\
$\stackrel{0}{0}$ \\
1
\end{tabular} & 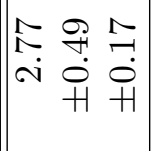 & 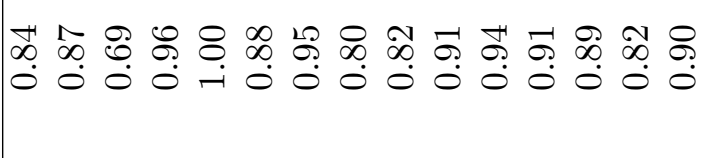 \\
\hline 0 & $\begin{array}{c}\stackrel{P}{0} \\
\stackrel{1}{1}\end{array}$ & 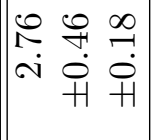 & 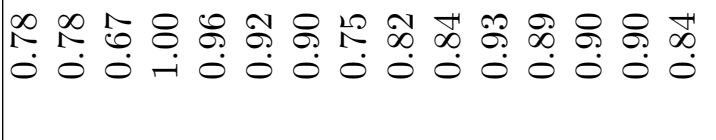 \\
\hline 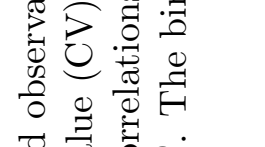 & 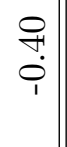 & 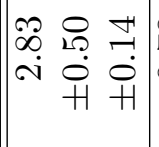 & 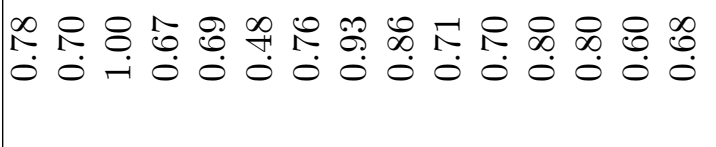 \\
\hline $\begin{array}{l}\infty \\
\pi \\
0 \\
0\end{array}$ & $\begin{array}{l}\stackrel{1}{r} \\
\dot{0} \\
1\end{array}$ & 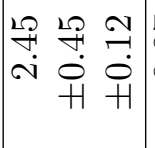 & 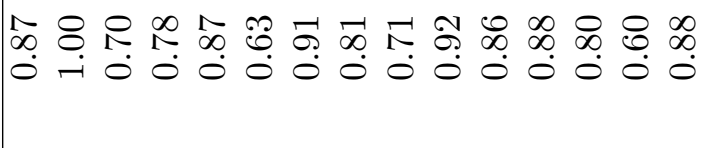 \\
\hline J & $\begin{array}{l}8 \\
\stackrel{8}{+} \\
i\end{array}$ & 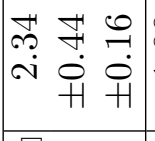 & 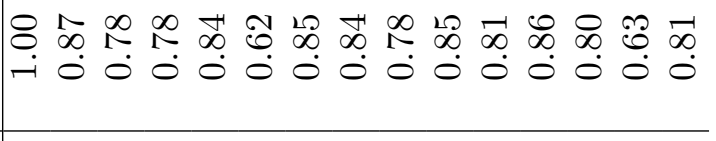 \\
\hline 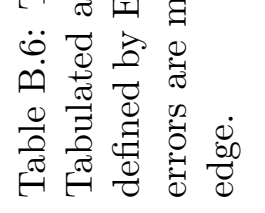 & $\begin{array}{c}0 \\
0 \\
0 \\
0 \\
.7 \\
0\end{array}$ & 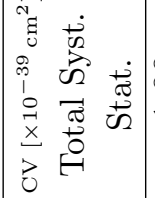 & 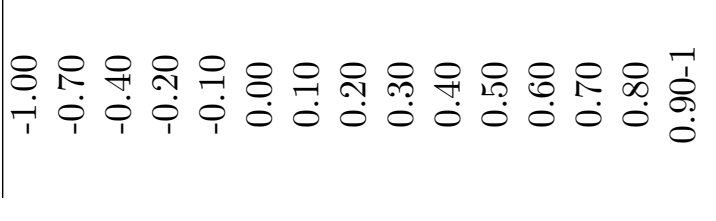 \\
\hline
\end{tabular}

